\title{
Palladium-Catalyzed $\beta-\mathrm{C}\left(\mathrm{sp}^{3}\right)-\mathrm{H}$ Nitrooxylation of Ketones and Amides Using Practical Oxidants
}

\author{
Yuan Xue, $\$$ Han Seul Park, $\$$ Chao Jiang, * and Jin-Quan Yu* \\ School of Chemistry and Chemical Engineering, Nanjing University of Science and Technology, \\ Nanjing, Jiangsu 210094, China. \\ Department of Chemistry, The Scripps Research Institute, 10550 N. Torrey Pines Road, La Jolla, \\ California 92037, United States \\ Email: chaojiang@njust.edu.cn, yu200@scripps.edu
}

Table of Contents

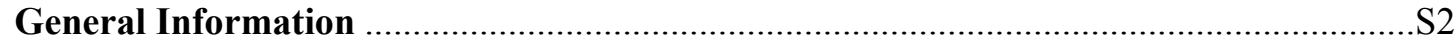

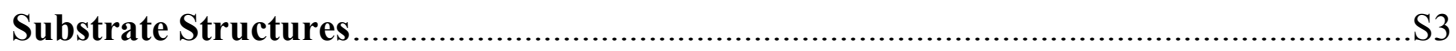

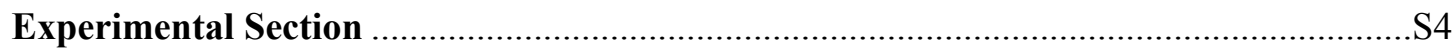

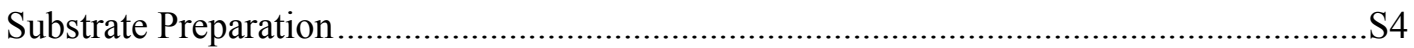

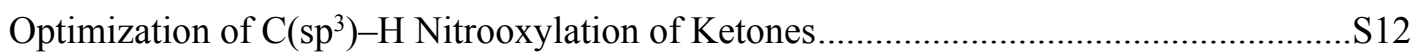

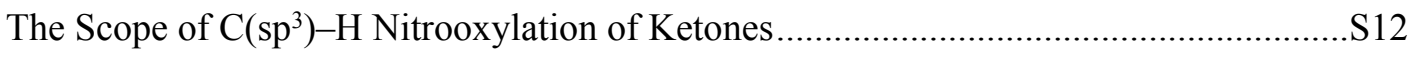

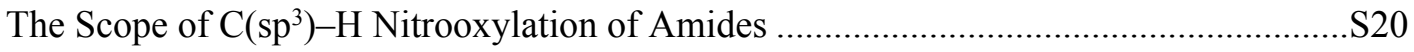

The Synthesis and Characterization of Palladacycles .......................................................S25

The Removal of Axiliary and Conversion of Nitrate .....................................................S27

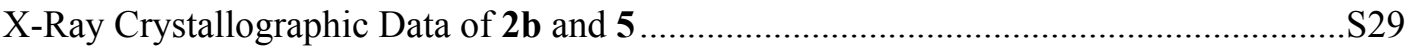

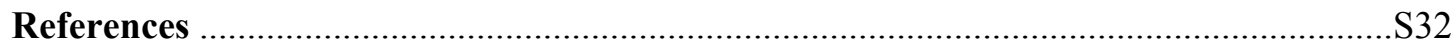

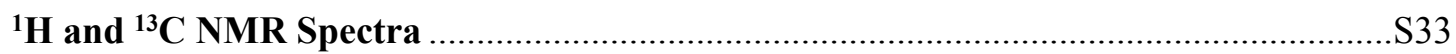




\section{General Information}

Ketones were obtained from the commercial sources or synthesized following literature procedures, and used to prepare the corresponding substrates. Amides were synthesized from corresponding carboxylic acids which are commercially available or prepared following literature procedures. Aminooxyacetic acid hemihydrochloride was obtained from Meryer, octafluorotluene was obtained from Fluorochem, $\mathrm{Pd}(\mathrm{OAc})_{2}$ was obtained from Heowns, $\mathrm{Fe}\left(\mathrm{NO}_{3}\right)_{3} \cdot 9 \mathrm{H}_{2} \mathrm{O}$ was obtained from Macklin, Hexamethyldisilane was from Meryer, HFIP (Hexafluoro-2-propanol) were obtained from Oakwood. $\mathrm{AgNO}_{3}$ was obtained from Chem-Impex Int'l. Inc. Solvents were obtainned from Nanjing Chemical Reagent and used directly without further purification. Analytical thin layer chromatography was performed on $0.5 \mathrm{~mm}$ silica gel. Visualization was carried out with UV light and Vogel's permanganate. ${ }^{1} \mathrm{H}$ NMR was recorded on Bruker AV-500 instrument (500 MHz), Bruker AMX-400 instrument (400 $\mathrm{MHz})$ or Bruker DRX-600 instrument $(600 \mathrm{MHz})$. Chemical shifts were quoted in parts per million $(\mathrm{ppm})$ referenced to $0.0 \mathrm{ppm}$ for tetramethylsilane. The following abbreviations (or combinations thereof) were used to explain multiplicities: $\mathrm{s}=$ singlet, $\mathrm{d}=$ doublet, $\mathrm{t}=$ triplet, $\mathrm{q}=$ quartet, $\mathrm{m}=$ multiplet, $\mathrm{br}=$ broad. Coupling constants, $J$, were reported in Hertz unit (Hz). ${ }^{13} \mathrm{C}$ NMR spectra were recorded on Bruker AV-500 instrument (126 MHz) ) or Bruker DRX-600 instrument (150 MHz) and were fully decoupled by broad band proton decoupling. Chemical shifts were reported in ppm referenced to either the center line of a triplet at $77.16 \mathrm{ppm}$ of chloroform- $d$ or the center line of a multiplet at $29.84 \mathrm{ppm}$ of acetone- $d 6$. In the ${ }^{13} \mathrm{C}$ NMR analysis, peaks that correspond to those of the polyfluoroarylamide auxiliary appeared as nearly invisible, complex sets of multiplets; they were omitted in the following spectroscopic analysis. High-resolution mass spectra (HRMS) were recorded on an Agilent Mass spectrometer using ESI-TOF (electrospray ionization-time of flight). Melting points were recorded on Stuart SMP50 automatic melting point apparatus or SGW X-4 Micro melting point instrument. 


\section{Substrate Structures}
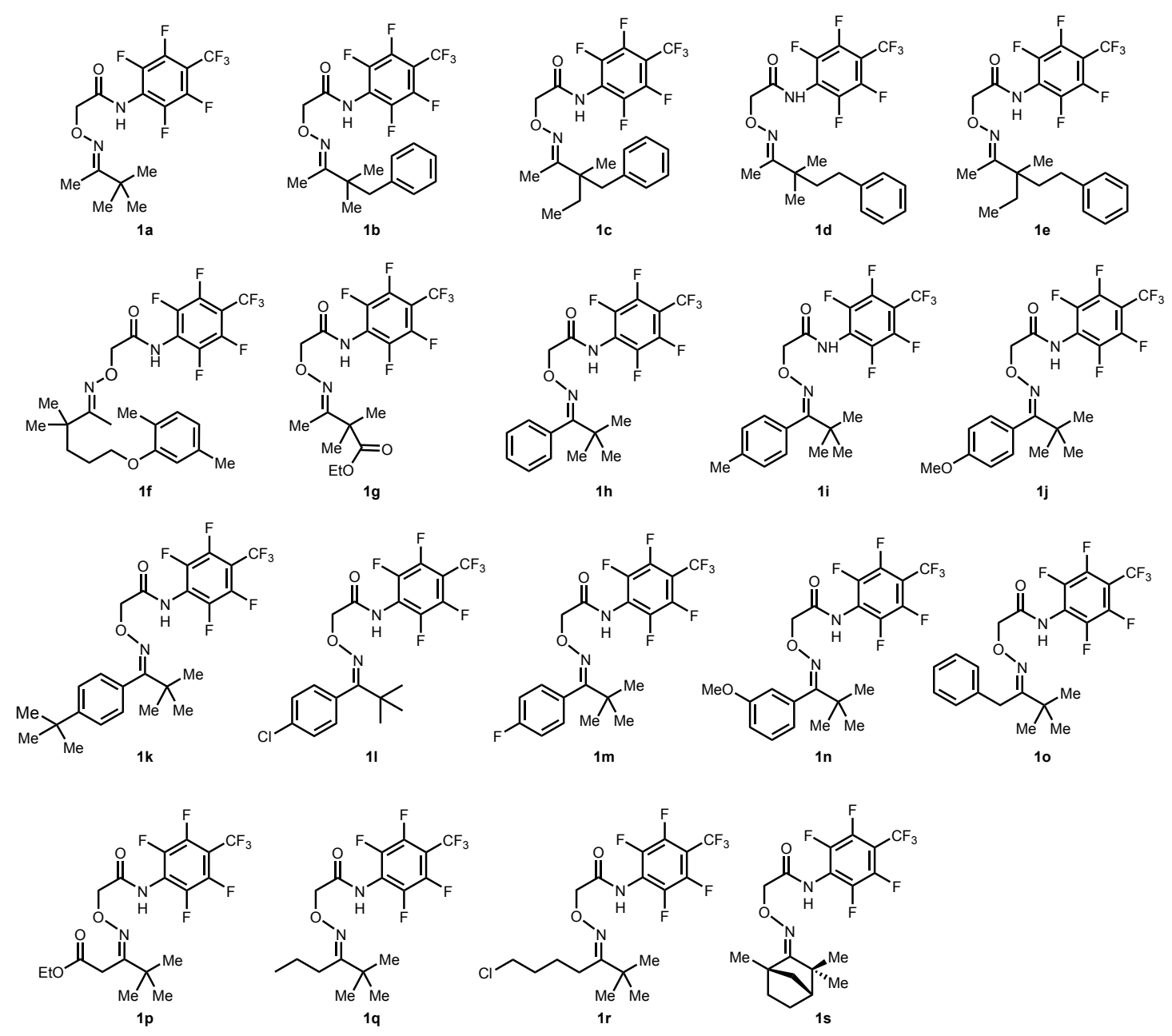

$$
\underbrace{M e}_{3 a} \underbrace{M}_{3 b}
$$

(1)

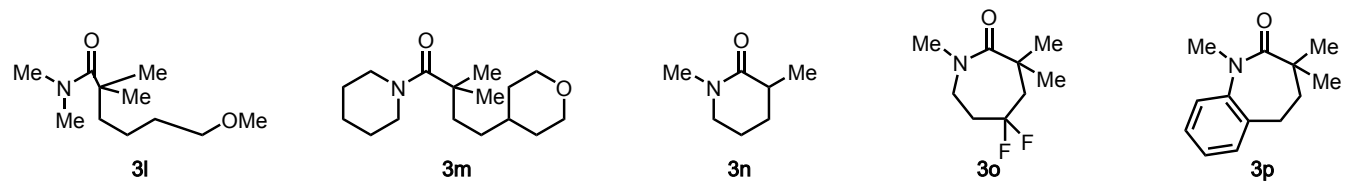




\section{Experimental Section}

\section{Substrate Preparation}

\section{Synthesis of ketone substrates}

Ketones for substrates $\mathbf{1 a}, \mathbf{1 h}, \mathbf{1 p}$, and $\mathbf{1 s}$ are commercial available. Ketones for substrates $\mathbf{1 b}, \mathbf{1 c}, \mathbf{1 d}$ and $\mathbf{1 e}$ were synthesized following literature procedures. ${ }^{1}$ Ketones for substrates 1f, and $\mathbf{1 g}$ were synthesized by the reaction between corresponding carboxylic acids and lithium methide. The procedure can be found in literature. ${ }^{2}$ Ketone for substrate 1i, $1 \mathbf{k}, \mathbf{1}, \mathbf{1} \mathbf{m}, \mathbf{1 n}$ was synthesized following literature procedures ${ }^{3}$. Ketone for substrate $\mathbf{1 j}$ was synthesized following literature procedures. ${ }^{4}$ Ketone for substrate 1o, 1q and 1r was synthesized following literature procedures. ${ }^{5}$

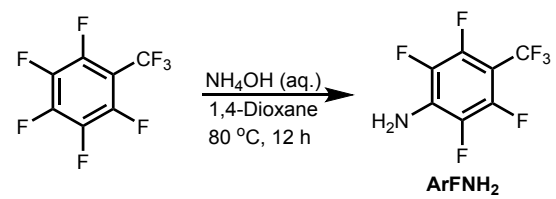

Preparation of 2,3,5,6-Tetrafluoro-4-(trifluoromethyl)aniline: ${ }^{6}$ To a solution of octafluorotoluene $(30 \mathrm{~mL})$ in 1,4-dioxane $(79 \mathrm{~mL})$ was added aq. $\mathrm{NH}_{4} \mathrm{OH}(53 \mathrm{~mL}$, $28 \% \mathrm{w} / \mathrm{w}$ ), and the reaction flask was sealed and heated to $80{ }^{\circ} \mathrm{C}$ in oil bath. The reaction mixture was stirred for $12 \mathrm{~h}$. The mixture was then cooled, and 1,4-dioxane was removed in vacuo. The resultant mixture was extracted with ethyl acetate $(3 \times 100$ $\mathrm{mL}$ ), and then concentrated in vacuo. The crude mixture was purified by column chromatography (hexanes : ethyl acetate $=4: 1$ ) to afford 2,3,5,6-tetrafluoro-4(trifluoromethyl)aniline as a colorless liquid (29.6 g, 60\%).

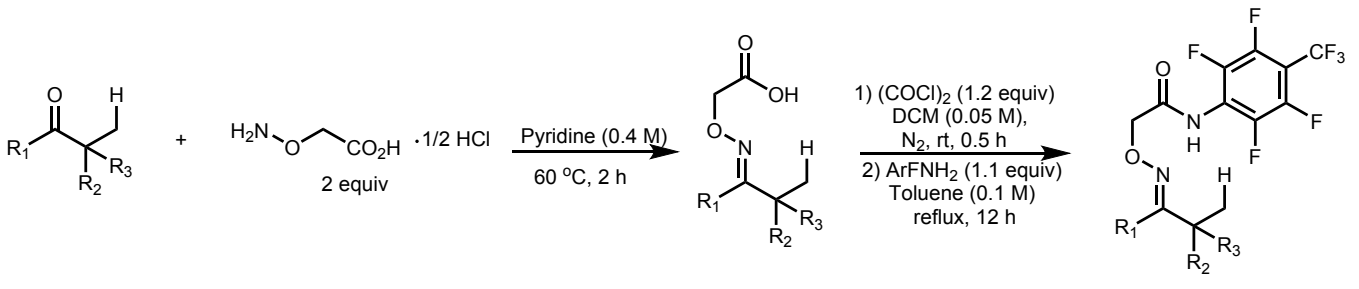

General Procedure for the Preparation of Substrates : Step1: Ketone (2 mmol, 1 equiv) and aminooxyacetic acid hemihydrochloride ( $4 \mathrm{mmol}, 437 \mathrm{mg}, 2$ equiv) were weighed into an oven dried $50 \mathrm{~mL}$ round bottom flask with a magnetic stir bar under air. $5 \mathrm{~mL}$ Pyridine was added and the mixture was stirred at $60^{\circ} \mathrm{C}$ in oil bath for $2 \mathrm{~h}$. Upon completion, most pyridine was evaporated under vacuum. The resulting mixture was diluted with EtOAc $(50 \mathrm{~mL})$ and washed successively with water $(100 \mathrm{~mL})$ and diluted $\mathrm{HCl}$ aqueous solution $(100 \mathrm{~mL}$, ca. $0.01 \mathrm{M})$. The organic phase was dried with anhydrous $\mathrm{Na}_{2} \mathrm{SO}_{4}$ and the solvent was removed under vacuum. Notably, the pure compounds were obtained in good yields for all cases without chromatography.

Step 2: An acid chloride $(1.2 \mathrm{mmol})$, prepared from the corresponding carboxylic acid and oxalyl chloride, was added to a vigorously stirred solution of 2,3,5,6-tetrafluoro-4(trifluoromethyl)aniline $(1.1 \mathrm{mmol})$ in toluene $(3 \mathrm{~mL})$. The reaction mixture was stirred 
for $12 \mathrm{~h}$ under reflux in oil bath. The resultant mixture was extracted with ethyl acetate $(3 \times 100 \mathrm{~mL})$, and then concentrated in vacuo. The crude mixture was purified by column chromatography (hexanes : ethyl acetate $=9: 1$ ) to afford corresponding substrates.

\section{Synthesis of amide substrates}

Amide substrates were synthesized from corresponding carboxylic acids which were obtained from commercial sources or prepared following literature procedures. ${ }^{7-9}$

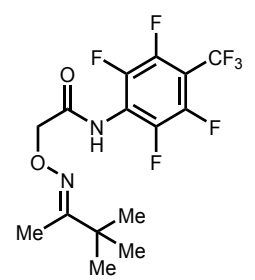

(E)-2-(((3,3-dimethylbutan-2-ylidene)amino)oxy)-N-(2,3,5,6-tetrafluoro-4-(trifluo romethyl)phenyl)acetamide (1a)

White solid (519.9 mg, 67\%), m.p. $64{ }^{\circ} \mathrm{C} .{ }^{1} \mathrm{H}$ NMR (500 MHz, $\left.\mathrm{CDCl}_{3}\right) \delta 8.13(\mathrm{~s}, 1 \mathrm{H})$, $4.68(\mathrm{~s}, 2 \mathrm{H}), 1.94(\mathrm{~s}, 3 \mathrm{H}), 1.15(\mathrm{~s}, 9 \mathrm{H}) .{ }^{13} \mathrm{C} \mathrm{NMR}\left(126 \mathrm{MHz}, \mathrm{CDCl}_{3}\right) \delta$ 168.8, 168.6, 72.3, 37.6, 27.4, 11.0. HRMS (ESI-TOF) Calcd for $\mathrm{C}_{15} \mathrm{H}_{14} \mathrm{~F}_{7} \mathrm{~N}_{2} \mathrm{O}_{2}^{-}[\mathrm{M}-\mathrm{H}]-: 387.0944$, found: 387.0954 .

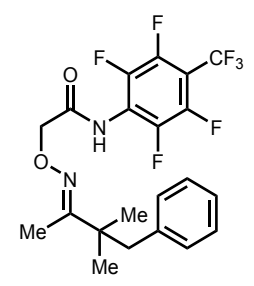

(E)-2-(((3,3-dimethyl-4-phenylbutan-2-ylidene)amino)oxy)-N-(2,3,5,6-tetrafluoro4-(trifluoromethyl)phenyl)acetamide (1b)

White solid (555.6 mg, 60\%), m.p. $91{ }^{\circ} \mathrm{C} .{ }^{1} \mathrm{H}$ NMR (500 MHz, $\left.\mathrm{CDCl}_{3}\right) \delta 7.69(\mathrm{~s}, 1 \mathrm{H})$, $7.10(\mathrm{~d}, J=7.6 \mathrm{~Hz}, 2 \mathrm{H}), 7.02(\mathrm{~d}, J=7.2 \mathrm{~Hz}, 2 \mathrm{H}), 6.86(\mathrm{t}, J=7.4 \mathrm{~Hz}, 1 \mathrm{H}), 4.60$ (s, 2H), $2.76(\mathrm{~s}, 2 \mathrm{H}), 2.05(\mathrm{~s}, 3 \mathrm{H}), 1.16(\mathrm{~s}, 6 \mathrm{H}) .{ }^{13} \mathrm{C} \mathrm{NMR}\left(126 \mathrm{MHz}, \mathrm{CDCl}_{3}\right) \delta$ 169.0, 166.8, 137.9, 130.1, 127.7, 125.9, 72.1, 46.3, 42.0, 25.2, 12.0. HRMS (ESI-TOF) Calcd for: $\mathrm{C}_{21} \mathrm{H}_{18} \mathrm{~F}_{7} \mathrm{~N}_{2} \mathrm{O}_{2}^{-}[\mathrm{M}-\mathrm{H}]^{-:}$463.1257, found: 463.1270 .<smiles>CCC(CC)(NC(=O)COC(=O)Nc1c(F)c(F)c(F)c(F)c1F)C(C)=O</smiles>

(E)-2-(((3-benzyl-3-methylpentan-2-ylidene)amino)oxy)-N-(2,3,5,6-tetrafluoro-4-( trifluoromethyl)phenyl)acetamide (1c) 
White solid (621.4 mg, 65\%), m.p. $101{ }^{\circ} \mathrm{C} .{ }^{1} \mathrm{H} \mathrm{NMR}\left(500 \mathrm{MHz}, \mathrm{CDCl}_{3}\right) \delta 7.67(\mathrm{~s}, 1 \mathrm{H})$, $7.09(\mathrm{~d}, J=7.6 \mathrm{~Hz}, 2 \mathrm{H}), 7.01(\mathrm{~d}, J=7.1 \mathrm{~Hz}, 2 \mathrm{H}), 6.84(\mathrm{t}, J=7.4 \mathrm{~Hz}, 1 \mathrm{H}), 4.61(\mathrm{~d}, J=$ $5.2 \mathrm{~Hz}, 2 \mathrm{H}), 2.84(\mathrm{~s}, 1 \mathrm{H}), 2.65(\mathrm{~d}, J=13.6 \mathrm{~Hz}, 1 \mathrm{H}), 2.03(\mathrm{~s}, 3 \mathrm{H}), 1.76(\mathrm{dq}, J=14.7$, $7.5 \mathrm{~Hz}, 1 \mathrm{H}), 1.49-1.41(\mathrm{~m}, 1 \mathrm{H}), 1.05(\mathrm{~s}, 3 \mathrm{H}), 0.84(\mathrm{t}, \mathrm{J}=7.5 \mathrm{~Hz}, 3 \mathrm{H}) .{ }^{13} \mathrm{C}$ NMR $(126$ $\left.\mathrm{MHz}, \mathrm{CDCl}_{3}\right) \delta 169.1,165.8,137.8,130.2,127.7,125.9,72.1,45.7,45.5,31.5,20.3$, 11.9, 8.7. HRMS (ESI-TOF) Calcd for $\mathrm{C}_{22} \mathrm{H}_{20} \mathrm{~F}_{7} \mathrm{~N}_{2} \mathrm{O}_{2}^{-}[\mathrm{M}-\mathrm{H}]:$ : 477.1413, found: 477.1427 .<smiles>CC(C)(C)C(C)(C)NOCC(=O)Nc1c(F)c(F)c(F)c(F)c1F</smiles>

(E)-2-(((3,3-dimethyl-5-phenylpentan-2-ylidene)amino)oxy)-N-(2,3,5,6-tetrafluor o-4-(trifluoromethyl)phenyl)acetamide (1d)

White solid (525.8 mg, 55\%), m.p. $55{ }^{\circ} \mathrm{C} .{ }^{1} \mathrm{H}$ NMR (500 MHz, $\left.\mathrm{CDCl}_{3}\right) \delta 8.08(\mathrm{~s}, 1 \mathrm{H})$, $7.26(\mathrm{~s}, 2 \mathrm{H}), 7.18(\mathrm{t}, J=7.4 \mathrm{~Hz}, 1 \mathrm{H}), 7.13(\mathrm{~d}, J=7.0 \mathrm{~Hz}, 2 \mathrm{H}), 4.72(\mathrm{~s}, 2 \mathrm{H}), 2.50-2.42$ $(\mathrm{m}, 2 \mathrm{H}), 1.94(\mathrm{~s}, 3 \mathrm{H}), 1.78(\mathrm{~d}, J=17.4 \mathrm{~Hz}, 2 \mathrm{H}), 1.20(\mathrm{~s}, 6 \mathrm{H}) .{ }^{13} \mathrm{C}$ NMR (126 MHz, $\left.\mathrm{CDCl}_{3}\right) \delta 168.7,167.3,142.1,128.5,128.2,126.0,72.4,42.3,40.9,31.2,25.5,11.1$. HRMS (ESI-TOF) Calcd for $\mathrm{C}_{22} \mathrm{H}_{20} \mathrm{~F}_{7} \mathrm{~N}_{2} \mathrm{O}_{2}{ }^{-}[\mathrm{M}-\mathrm{H}]^{-}: 477.1413$, found: 477.1424 .<smiles>CCCC(CC)(CCc1ccccc1)C(=O)NCC(=O)Nc1c(F)c(F)c(F)c(F)c1F</smiles>

(E)-2-(((3-ethyl-3-methyl-5-phenylpentan-2-ylidene)amino)oxy)-N-(2,3,5,6-tetrafl uoro-4-(trifluoromethyl)phenyl)acetamide (1e)

White solid (502.2 mg, 51\%), m.p. $122{ }^{\circ} \mathrm{C} .{ }^{1} \mathrm{H}$ NMR (500 MHz, $\left.\mathrm{CDCl}_{3}\right) \delta 8.10(\mathrm{~s}, 1 \mathrm{H})$, $7.29-7.26(\mathrm{~m}, 2 \mathrm{H}), 7.19(\mathrm{~d}, J=7.3 \mathrm{~Hz}, 1 \mathrm{H}), 7.14(\mathrm{~d}, J=7.3 \mathrm{~Hz}, 2 \mathrm{H}), 4.73(\mathrm{~s}, 2 \mathrm{H})$, $2.56(\mathrm{td}, J=12.9,5.1 \mathrm{~Hz}, 1 \mathrm{H}), 2.37(\mathrm{td}, J=13.0,4.7 \mathrm{~Hz}, 1 \mathrm{H}), 1.92(\mathrm{~s}, 3 \mathrm{H}), 1.87-1.79$ $(\mathrm{m}, 1 \mathrm{H}), 1.73-1.66(\mathrm{~m}, 1 \mathrm{H}), 1.62(\mathrm{dd}, J=14.2,7.3 \mathrm{~Hz}, 1 \mathrm{H}), 1.50(\mathrm{dd}, J=14.1,7.5$ $\mathrm{Hz}, 1 \mathrm{H}), 1.18(\mathrm{~s}, 3 \mathrm{H}), 0.81(\mathrm{t}, J=7.5 \mathrm{~Hz}, 3 \mathrm{H}) .{ }^{13} \mathrm{C} \mathrm{NMR}\left(126 \mathrm{MHz}, \mathrm{CDCl}_{3}\right) \delta 168.7$, 166.4, 142.2, 128.5, 128.2, 126.0, 72.5, 44.4, 41.0, 31.6, 30.9, 20.6, 11.1, 8.5. HRMS (ESI-TOF) Calcd for $\mathrm{C}_{23} \mathrm{H}_{22} \mathrm{~F}_{7} \mathrm{~N}_{2} \mathrm{O}_{2}{ }^{-}[\mathrm{M}-\mathrm{H}]$ ]: 491.1570, found: 491.1583.

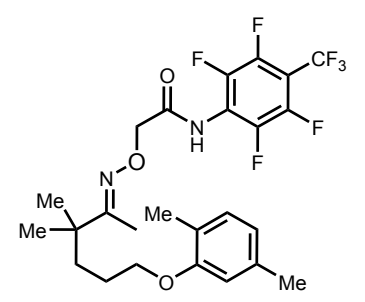


(E)-2-(((6-(2,5-dimethylphenoxy)-3,3-dimethylhexan-2-ylidene)amino)oxy)-N-(2,3,5 ,6-tetrafluoro-4-(trifluoromethyl)phenyl)acetamide (1f)

White solid (632.5 mg, 59\%), m.p. $72{ }^{\circ} \mathrm{C} .{ }^{1} \mathrm{H} \mathrm{NMR}\left(500 \mathrm{MHz}, \mathrm{CDCl}_{3}\right) \delta 8.06(\mathrm{~s}, 1 \mathrm{H})$, $6.98(\mathrm{~d}, J=7.5 \mathrm{~Hz}, 1 \mathrm{H}), 6.65(\mathrm{~d}, J=7.5 \mathrm{~Hz}, 1 \mathrm{H}), 6.57(\mathrm{~s}, 1 \mathrm{H}), 4.70(\mathrm{~s}, 2 \mathrm{H}), 3.89$ (s, 2H), $2.30(\mathrm{~s}, 3 \mathrm{H}), 2.14(\mathrm{~s}, 3 \mathrm{H}), 1.95(\mathrm{~s}, 3 \mathrm{H}), 1.67(\mathrm{~s}, 4 \mathrm{H}), 1.17(\mathrm{~s}, 6 \mathrm{H}) \cdot{ }^{13} \mathrm{C}$ NMR $(126$ $\left.\mathrm{MHz}, \mathrm{CDCl}_{3}\right) \delta 168.7,167.4,156.8,136.4,130.4,123.4,120.8,111.8,67.7,40.6,36.5$, 25.5, 24.9, 21.4, 15.7, 11.0. HRMS (ESI-TOF) Calcd for $\mathrm{C}_{26} \mathrm{H}_{28} \mathrm{~F}_{7} \mathrm{~N}_{2} \mathrm{O}_{2}^{-}$[M-H]: 535.1832, found: 535.1845 .

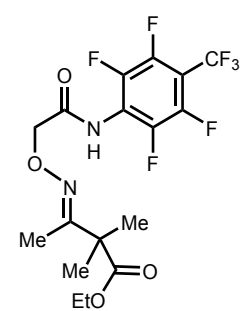

(E)-2,2-dimethyl-3-((2-oxo-2-((2,3,5,6-tetrafluoro-4-(trifluoromethyl)phenyl)amin o)ethoxy)imino)butanoate) ethyl (1g)

White solid (562.0 mg, 63\%), m.p. $62{ }^{\circ} \mathrm{C} .1 \mathrm{H} \mathrm{NMR}(500 \mathrm{MHz}, \mathrm{CDCl}) \delta 8.00(\mathrm{~s}, 1 \mathrm{H}), 4.74$ $(\mathrm{s}, 2 \mathrm{H}), 4.17(\mathrm{~d}, J=7.1 \mathrm{~Hz}, 2 \mathrm{H}), 1.94(\mathrm{~s}, 3 \mathrm{H}), 1.41(\mathrm{~s}, 6 \mathrm{H}), 1.24(\mathrm{t}, J=7.1 \mathrm{~Hz}, 3 \mathrm{H}) .{ }^{13} \mathrm{C} \mathrm{NMR}$ $\left(126 \mathrm{MHz}, \mathrm{CDCl}_{3}\right) \delta 174.5,168.4,163.2,72.6,61.4,49.3,23.0,14.0,12.8$. HRMS (ESI-TOF) Calcd for $\mathrm{C}_{17} \mathrm{H}_{16} \mathrm{~F}_{7} \mathrm{~N}_{2} \mathrm{O}_{2}{ }^{-}[\mathrm{M}-\mathrm{H}]^{-}:$:45.0999, found: 445.1009 .<smiles>CC(C)(C)C(=NOCC(=O)Nc1c(F)c(F)c(F)c(F)c1F)c1ccccc1</smiles>

(Z)-2-(((2,2-dimethyl-1-phenylpropylidene)amino)oxy)-N-(2,3,5,6-tetrafluoro-4-(t rifluoromethyl)phenyl)acetamide (1h)

White solid (522.0 mg, 58\%), m.p. $107{ }^{\circ} \mathrm{C} .{ }^{1} \mathrm{H}$ NMR (500 MHz, $\left.\mathrm{CDCl}_{3}\right) \delta 7.68(\mathrm{~s}, 1 \mathrm{H})$, $7.47-7.35(\mathrm{~m}, 3 \mathrm{H}), 7.13-7.04(\mathrm{~m}, 2 \mathrm{H}), 4.65(\mathrm{~s}, 2 \mathrm{H}), 1.20(\mathrm{~s}, 9 \mathrm{H}) .{ }^{13} \mathrm{C}$ NMR $(126$ $\left.\mathrm{MHz}, \mathrm{CDCl}_{3}\right) \delta 170.5,168.1,133.5,128.4,126.8,72.6,37.8,28.1$. HRMS (ESI-TOF) Calcd for $\mathrm{C}_{20} \mathrm{H}_{16} \mathrm{~F}_{7} \mathrm{~N}_{2} \mathrm{O}_{2}{ }^{-}[\mathrm{M}-\mathrm{H}]^{-}:$:449.1100, found: 449.1112 .<smiles>Cc1ccc(C(=NOCC(=O)Nc2c(F)c(F)c(F)c(F)c2F)C(C)(C)C)cc1</smiles>

(Z)-2-(((2,2-dimethyl-1-(p-tolyl)propylidene)amino)oxy)-N-(2,3,5-trifluoro-4-(trif luoromethyl)phenyl)acetamide (1i)

White solid (464.0 mg, 50\%), m.p. $110{ }^{\circ} \mathrm{C} .{ }^{1} \mathrm{H}$ NMR (500 MHz, $\left.\mathrm{CDCl}_{3}\right) \delta 7.75$ (s, 1H), 
$7.26(\mathrm{~d}, J=7.6 \mathrm{~Hz}, 2 \mathrm{H}), 6.98(\mathrm{~d}, J=7.9 \mathrm{~Hz}, 2 \mathrm{H}), 4.65(\mathrm{~s}, 2 \mathrm{H}), 2.39$ (s, 3H) 1.20 (s, 9H). ${ }^{13} \mathrm{C} \mathrm{NMR}\left(126 \mathrm{MHz}, \mathrm{CDCl}_{3}\right) \delta 170.7,168.2,138.3,130.4,129.1,126.7,72.6,37.9$, 28.1, 21.3 HRMS (ESI-TOF) Calcd for $\mathrm{C}_{21} \mathrm{H}_{18} \mathrm{~F}_{7} \mathrm{~N}_{2} \mathrm{O}_{2}^{-}$[M-H]: 463.1257, found: 463.1267 .<smiles>COc1ccc(C(=NOCC(=O)Nc2c(F)c(F)c(F)c(F)c2F)C(C)(C)C)cc1</smiles>

(Z)-2-(((1-(4-methoxyphenyl)-2,2-dimethylpropylidene)amino)oxy)-N-(2,3,5,6-tet rafluoro-4-(trifluoromethyl)phenyl)acetamide (1j)

White solid (547.1 mg, 57\%), m.p. $74{ }^{\circ} \mathrm{C} .{ }^{1} \mathrm{H} \mathrm{NMR}\left(500 \mathrm{MHz}, \mathrm{CDCl}_{3}\right) \delta 7.75(\mathrm{~s}, 1 \mathrm{H})$, $7.04(\mathrm{~d}, J=8.8 \mathrm{~Hz}, 2 \mathrm{H}), 6.98(\mathrm{~d}, J=8.8 \mathrm{~Hz}, 2 \mathrm{H}), 4.67(\mathrm{~s}, 2 \mathrm{H}), 3.86(\mathrm{~s}, 3 \mathrm{H}), 1.22$ (s, 9H). ${ }^{13} \mathrm{C} \mathrm{NMR}\left(126 \mathrm{MHz}, \mathrm{CDCl}_{3}\right) \delta 170.4,168.1,159.5,128.2,125.5,113.9,72.6,55.2$, 38.0, 28.1. HRMS (ESI-TOF) Calcd for $\mathrm{C}_{21} \mathrm{H}_{18} \mathrm{~F}_{7} \mathrm{~N}_{2} \mathrm{O}_{3}^{-}[\mathrm{M}-\mathrm{H}]^{-}: 479.1206$, found: 479.1216 .<smiles>CC(C)(C)C(=NOCC(=O)Nc1c(F)c(F)c(F)c(F)c1F)c1ccc(C(C)(C)C)cc1</smiles>

(Z)-2-(((1-(4-(tert-butyl)phenyl)-2,2-dimethylpropylidene)amino)oxy)-N-(2,3,5,6-t etrafluoro-4-(trifluoromethyl)phenyl)acetamide (1k)

White solid (526.2 mg, 52\%), m.p. $80{ }^{\circ} \mathrm{C} .{ }^{1} \mathrm{H} \mathrm{NMR}\left(500 \mathrm{MHz}, \mathrm{CDCl}_{3}\right) \delta 7.73(\mathrm{~s}, 1 \mathrm{H})$, $7.44(\mathrm{~d}, J=8.4 \mathrm{~Hz}, 2 \mathrm{H}), 7.01$ (d, $J=8.4 \mathrm{~Hz}, 2 \mathrm{H}), 4.65$ (s, 2H), 1.34 (s, 9H), 1.20 (s, 9H). ${ }^{13} \mathrm{C} \mathrm{NMR}\left(126 \mathrm{MHz}, \mathrm{CDCl}_{3}\right) \delta 170.7,168.1,151.3,130.5,126.5,125.3,72.5,37.9$, 34.7, 31.3, 28.1. HRMS (ESI-TOF) Calcd for $\mathrm{C}_{24} \mathrm{H}_{24} \mathrm{~F}_{7} \mathrm{~N}_{2} \mathrm{O}_{2}{ }^{-}[\mathrm{M}-\mathrm{H}]^{-}: 505.1726$, found: 505.1740 .<smiles>CC(C)(C)/C(=N\OCC(=O)Nc1c(F)c(F)c(F)c(F)c1F)c1ccc(Cl)cc1</smiles>

(Z)-2-(((1-(4-chlorophenyl)-2,2-dimethylpropylidene)amino)oxy)-N-(2,3,5,6-tetraf luoro-4-(trifluoromethyl)phenyl)acetamide (1I)

Yellow solid (580.8 mg, 60\%), m.p. $104{ }^{\circ} \mathrm{C} .{ }^{1} \mathrm{H}$ NMR (500 MHz, $\left.\mathrm{CDCl}_{3}\right) \delta 7.65$ (s, $1 \mathrm{H}), 7.42(d, \mathrm{~J}=8.4 \mathrm{~Hz}, 2 \mathrm{H}), 7.03(\mathrm{~d}, J=8.4 \mathrm{~Hz}, 2 \mathrm{H}), 4.65(\mathrm{~s}, 2 \mathrm{H}), 1.19(\mathrm{~s}, 9 \mathrm{H}) .{ }^{13} \mathrm{C}$ 
NMR $\left(126 \mathrm{MHz}, \mathrm{CDCl}_{3}\right) \delta 169.5,167.7,134.6,131.7,128.8,128.4,72.7,37.8,28.0$. HRMS (ESI-TOF) Calcd for $\mathrm{C}_{20} \mathrm{H}_{15} \mathrm{ClF}_{7} \mathrm{~N}_{2} \mathrm{O}_{2}{ }^{-}[\mathrm{M}-\mathrm{H}]^{-}: 483.0711$, found: 483.0723 .<smiles>CC(C)(C)C(C)(C)N=C(NC(=O)CONc1c(F)c(F)c(F)c(F)c1F)c1ccc(F)cc1</smiles>

(Z)-2-(((1-(4-fluorophenyl)-2,2-dimethylpropylidene)amino)oxy)-N-(2,3,5,6-tetraf luoro-4-(trifluoromethyl)phenyl)acetamide (1m)

White solid (526.3 mg, 49\%), m.p. $60{ }^{\circ} \mathrm{C} .{ }^{1} \mathrm{H} \mathrm{NMR}\left(500 \mathrm{MHz}, \mathrm{CDCl}_{3}\right) \delta 7.68(\mathrm{~s}, 1 \mathrm{H})$, $7.18-7.11(\mathrm{~m}, 2 \mathrm{H}), 7.11-7.04(\mathrm{~m}, 2 \mathrm{H}), 4.65(\mathrm{~s}, 2 \mathrm{H}), 1.19(\mathrm{~s}, 9 \mathrm{H}) .{ }^{13} \mathrm{C}$ NMR $(126$ $\left.\mathrm{MHz}, \mathrm{CDCl}_{3}\right) \delta 169.7,167.8,163.5,161.5,129.2,129.2,128.8,128.8,115.7,115.5$, 72.6, 37.9, 28.1. HRMS (ESI-TOF) Calcd for $\mathrm{C}_{20} \mathrm{H}_{15} \mathrm{~F}_{8} \mathrm{~N}_{2} \mathrm{O}_{2}^{-}[\mathrm{M}-\mathrm{H}]^{-}: 467.1006$, found: 467.1014 .<smiles>COc1cccc(C(=O)NC(=O)CONc2c(F)c(F)c(F)c(F)c2F)c1F</smiles>

(Z)-2-(((1-(3-methoxyphenyl)-2,2-dimethylpropylidene)amino)oxy)-N-(2,3,5,6-tet rafluoro-4-(trifluoromethyl)phenyl)acetamide (1n)

White solid (528.0 mg, 55\%), m.p. $66{ }^{\circ} \mathrm{C} .{ }^{1} \mathrm{H} \mathrm{NMR}\left(500 \mathrm{MHz}, \mathrm{CDCl}_{3}\right) \delta 7.72(\mathrm{~s}, 1 \mathrm{H})$, $7.36(\mathrm{t}, J=7.9 \mathrm{~Hz}, 1 \mathrm{H}), 6.93(\mathrm{dd}, J=8.3,2.1 \mathrm{~Hz}, 1 \mathrm{H}), 6.66(\mathrm{~d}, J=7.6 \mathrm{~Hz}, 1 \mathrm{H}), 6.64-$ $6.59(\mathrm{~m}, 1 \mathrm{H}), 4.66(\mathrm{~s}, 2 \mathrm{H}), 3.82(\mathrm{~s}, 3 \mathrm{H}), 1.21(\mathrm{~s}, 9 \mathrm{H}) .{ }^{13} \mathrm{C} \mathrm{NMR}\left(126 \mathrm{MHz}, \mathrm{CDCl}_{3}\right) \delta$ 170.2 , 168.0, 159.5, 134.8, 129.6, 119.0, 113.4, 113.0, 72.6, 55.3, 37.8, 28.1. HRMS (ESI-TOF) Calcd for $\mathrm{C}_{21} \mathrm{H}_{18} \mathrm{~F}_{7} \mathrm{~N}_{2} \mathrm{O}_{3}{ }^{-}[\mathrm{M}-\mathrm{H}]=:$ 479.1206, found: 479.1215.<smiles>CC(C)(C)C(C)(C)/C(=N\OCC(=O)Nc1c(F)c(F)c(F)c(F)c1F)OCc1ccccc1</smiles>

(E)-2-(((3,3-dimethyl-1-phenylbutan-2-ylidene)amino)oxy)-N-(2,3,5,6-tetrafluoro4-(trifluoromethyl)phenyl)acetamide (10)

White solid (483.0 mg, 52\%), m.p. $74{ }^{\circ} \mathrm{C} .{ }^{1} \mathrm{H}$ NMR $\left(500 \mathrm{MHz}, \mathrm{CDCl}_{3}\right) \delta 7.23(\mathrm{~d}, J=$ $6.6 \mathrm{~Hz}, 3 \mathrm{H}), 7.12-7.07(\mathrm{~m}, 2 \mathrm{H}), 4.66(\mathrm{~s}, 2 \mathrm{H}), 3.78(\mathrm{~s}, 2 \mathrm{H}), 1.23(\mathrm{~s}, 9 \mathrm{H}) .{ }^{13} \mathrm{C} \mathrm{NMR}$ $\left(126 \mathrm{MHz} \mathrm{CDCl}_{3}\right) \delta 168.3,137.4,128.7,128.0,126.4,72.6,37.9,32.5,27.9$. HRMS (ESI-TOF) Calcd for $\mathrm{C}_{21} \mathrm{H}_{18} \mathrm{~F}_{7} \mathrm{~N}_{2} \mathrm{O}_{2}^{-}$[M-H] : 463.1257, found: 463.1270 . 
<smiles>C[C@@H](N=C(O)CC(=O)OCC(=O)Nc1c(F)c(F)c(F)c(F)c1F)C(C)(C)C</smiles>

(E)-4,4-dimethyl-3-((2-0xo-2-((2,3,5,6-tetrafluoro-4-(trifluoromethyl)phenyl)ami no)ethoxy)imino)pentanoate ethyl (1p)

White solid (496.8 mg, 54\%), m.p. $50{ }^{\circ} \mathrm{C} .{ }^{1} \mathrm{H}$ NMR (500 MHz, $\left.\mathrm{CDCl}_{3}\right) \delta 9.24(\mathrm{~s}, 1 \mathrm{H})$, $4.77(\mathrm{~s}, 2 \mathrm{H}), 4.20(\mathrm{~d}, J=7.1 \mathrm{~Hz}, 2 \mathrm{H}), 3.48(\mathrm{~s}, 2 \mathrm{H}), 1.32-1.29(\mathrm{~m}, 3 \mathrm{H}), 1.16(\mathrm{~s}, 9 \mathrm{H})$. ${ }^{13} \mathrm{C}$ NMR $\left(126 \mathrm{MHz}, \mathrm{CDCl}_{3}\right) \delta 170.4,169.3,162.2,72.7,62.0,37.4,31.3,26.9,13.9$. HRMS (ESI-TOF) Calcd for $\mathrm{C}_{18} \mathrm{H}_{18} \mathrm{~F}_{7} \mathrm{~N}_{2} \mathrm{O}_{4}{ }^{-}[\mathrm{M}-\mathrm{H}]^{-:}: 459.1155$, found: 459.1168 .<smiles>CCC/C(=N\OCC(=O)Nc1c(F)c(F)c(F)c(F)c1F)C(C)(C)C</smiles>

(E)-2-(((2,2-dimethylhexan-3-ylidene)amino)oxy)-N-(2,3,5,6-tetrafluoro-4-(trifluo romethyl)phenyl)acetamide (1q)

White solid (582.4 mg, 70\%), m.p. $70{ }^{\circ} \mathrm{C} .{ }^{1} \mathrm{H}$ NMR $\left(500 \mathrm{MHz}, \mathrm{CDCl}_{3}\right) \delta 8.13(\mathrm{~s}, 1 \mathrm{H})$, $4.66(\mathrm{~s}, 2 \mathrm{H}), 2.35-2.27(\mathrm{~m}, 2 \mathrm{H}), 1.64-1.54(\mathrm{~m}, 2 \mathrm{H}), 1.15(\mathrm{~s}, 9 \mathrm{H}), 1.00(\mathrm{t}, J=7.4 \mathrm{~Hz}$, $3 \mathrm{H}) .{ }^{13} \mathrm{C} \mathrm{NMR}\left(126 \mathrm{MHz}, \mathrm{CDCl}_{3}\right) \delta 171.8,168.9,72.4,37.9,28.9,27.7,27.6,20.6$, 14.9. HRMS (ESI-TOF) Calcd for $\mathrm{C}_{17} \mathrm{H}_{20} \mathrm{~F}_{7} \mathrm{~N}_{2} \mathrm{O}_{2}{ }^{+}[\mathrm{M}+\mathrm{H}]^{+}: 417.1413$, found: 417.1405 .<smiles>CC(C)(C)C(C)(C)N=C(CCCCCl)Nc1c(F)c(F)c(F)c(F)c1F</smiles>

(E)-2-(((7-chloro-2,2-dimethylheptan-3-ylidene)amino)oxy)-N-(2,3,5,6-tetrafluoro -4-(trifluoromethyl)phenyl)acetamide (1r)

White solid (603.2 mg, 65\%), m.p. $75{ }^{\circ} \mathrm{C} .{ }^{1} \mathrm{H}$ NMR (500 MHz, $\mathrm{CDCl}_{3}$ ) $\delta 8.11$ (s), 4.67 (s), 3.57 (t, $J=5.0 \mathrm{~Hz}), 2.35$ (dd, $J=8.2,4.8 \mathrm{~Hz}), 1.85$ (td, $J=10.7,5.3 \mathrm{~Hz}), 1.73$ (dt, $J=15.8,6.1 \mathrm{~Hz}), 1.16(\mathrm{~s}, 9 \mathrm{H}) .{ }^{13} \mathrm{C} \mathrm{NMR}\left(126 \mathrm{MHz}, \mathrm{CDCl}_{3}\right) \delta 171.2,168.6,120.1,72.4$, 63.6, 44.3, 37.9, 33.4, 32.9, 31.9, 29.2, 27.6, 26.1, 25.8, 24.1, 22.3. HRMS (ESI-TOF) Calcd for $\mathrm{C}_{18} \mathrm{H}_{21} \mathrm{ClF}_{7} \mathrm{~N}_{2} \mathrm{O}_{2}{ }^{+}[\mathrm{M}+\mathrm{H}]^{+}$: 465.1180, found: 465.1174 . 


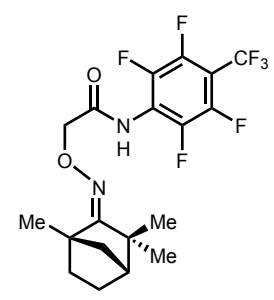

N-(2,3,5,6-tetrafluoro-4-(trifluoromethyl)phenyl)-2-((((1R,4S,E)-1,3,3-trimethylbi cyclo[2.2.1] heptan-2-ylidene)amino)oxy)acetamide (1s)

White solid (607.2 mg, 69\%), m.p. $75^{\circ} \mathrm{C} .{ }^{1} \mathrm{H} \mathrm{NMR}\left(500 \mathrm{MHz}, \mathrm{CDCl}_{3}\right) \delta 8.18(\mathrm{~s}, 1 \mathrm{H})$, $4.63(\mathrm{~s}, 2 \mathrm{H}), 1.88(\mathrm{~s}, 1 \mathrm{H}), 1.81(\mathrm{dd}, J=10.4,8.0 \mathrm{~Hz}, 1 \mathrm{H}), 1.75(\mathrm{dd}, J=10.4,1.8 \mathrm{~Hz}$, $1 \mathrm{H}), 1.62$ (dd, $J=8.0,1.6 \mathrm{~Hz}, 2 \mathrm{H}), 1.43$ (ddd, $J=7.9,6.9,1.6 \mathrm{~Hz}, 2 \mathrm{H}), 1.34$ (s, 3H), $1.31(\mathrm{~s}, 3 \mathrm{H}), 1.22(\mathrm{~s}, 3 \mathrm{H}) .{ }^{13} \mathrm{C} \mathrm{NMR}\left(126 \mathrm{MHz}, \mathrm{CDCl}_{3}\right) \delta 177.3,168.9,72.5,50.8,48.6$, 45.1, 43.4, 34.3, 25.2, 23.4, 22.7, 16.9. HRMS (ESI-TOF) Calcd for $\mathrm{C}_{19} \mathrm{H}_{18} \mathrm{~F}_{7} \mathrm{~N}_{2} \mathrm{O}_{2}{ }^{-}$[M$\mathrm{H}]:$ : 439.1257, found: 439.1269 . 


\section{Optimization of $\mathbf{C}\left(\mathrm{sp}^{3}\right)-\mathrm{H}$ Nitrooxylation of Ketones}

Table S1 Direct Group Screening ${ }^{a, b, c, d, e, f}$

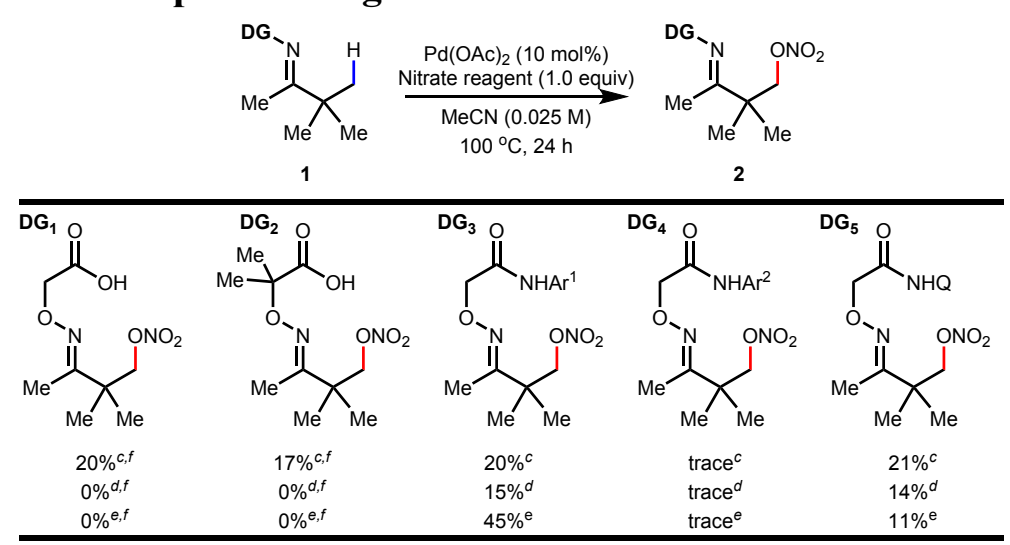

${ }^{a}$ Conditions: Substrate $\left(0.05 \mathrm{mmol}, 1.0\right.$ equiv), $\mathrm{Pd}(\mathrm{OAc})_{2}(10 \mathrm{~mol} \%)$, nitrate reagent (1.0 equiv), $\mathrm{MeCN}(2 \mathrm{~mL}), 100^{\circ} \mathrm{C}$, under air, $24 \mathrm{~h} . \mathrm{Ar}^{1}=4-\mathrm{CF}_{3}\left(\mathrm{C}_{6} \mathrm{~F}_{4}\right), \mathrm{Ar}^{2}=\mathrm{C}_{6} \mathrm{~F}_{5}, \mathrm{Q}$ $=$ quinoline. ${ }^{b}$ Isolated yields, best results for each $\mathrm{T}$ with different nitrate reagents were given. ${ }^{c} \mathrm{TBN}$ (1.0 equiv). ${ }^{d} \mathrm{Al}\left(\mathrm{NO}_{3}\right)_{3} \cdot 9 \mathrm{H}_{2} \mathrm{O}$ (1.0 equiv). ${ }^{e} \mathrm{Fe}\left(\mathrm{NO}_{3}\right)_{3} \cdot 9 \mathrm{H}_{2} \mathrm{O}$ (1.0 equiv). ${ }^{f}$ After the reaction, the carboxylic acid is converted to corresponding phenylamide for convenient chromatographic purification.

\section{The Scope of C( $\left.\mathrm{sp}^{3}\right)-\mathrm{H}$ Nitrooxylation of Ketones}

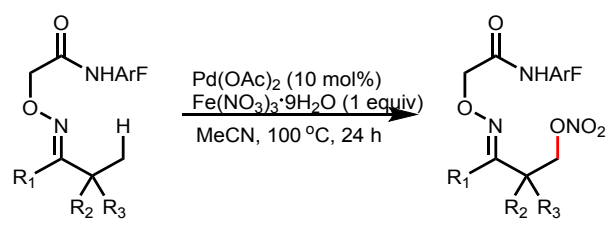

General Procedures : Substrate $1(0.05 \mathrm{mmol}), \mathrm{Pd}(\mathrm{OAc})_{2}(0.005 \mathrm{mmol}, 1.12 \mathrm{mg})$, and $\mathrm{Fe}\left(\mathrm{NO}_{3}\right)_{3} \cdot 9 \mathrm{H}_{2} \mathrm{O}(0.05 \mathrm{mmol}, 20.2 \mathrm{mg})$ were weighed into a reaction vial $(10 \mathrm{~mL})$ with a magnetic stir bar under air. MeCN $(2 \mathrm{~mL})$ was added, and the vial was sealed with a cap. The reaction mixture was stirred at $110{ }^{\circ} \mathrm{C}$ in oil bath for 24 hours. Upon completion, the reaction mixture was cooled to room temperature and diluted with EtOAc. Then the reaction mixture was filtered through a plug of silicon gel .The solvent was removed under vacuum. The resulting mixture was purified by preparative thinlayer chromatography.<smiles>CC(=O)C(C)(C)CN1CC(=O)Nc2c(F)c(F)c(F)c(F)c2O1</smiles> 
(E)-2,2-dimethyl-3-((2-oxo-2-((2,3,5,6-tetrafluoro-4-(trifluoromethyl)phenyl)amin o)ethoxy)imino)butyl nitrate (2a)

Substrate 1a was nitrooxylated following the general nitrooxylation procedure. After purification by preparative thin-layer chromatography (Hexane : EtOAc $=6: 1$ as eluent), 2a was obtained as a white solid (18.2 mg, 81\%), m.p. $47{ }^{\circ} \mathrm{C} .{ }^{1} \mathrm{H}$ NMR $(500 \mathrm{MHz}$, $\left.\mathrm{CDCl}_{3}\right) \delta 7.85(\mathrm{~s}, 1 \mathrm{H}), 4.72(\mathrm{~s}, 2 \mathrm{H}), 4.49(\mathrm{~s}, 2 \mathrm{H}), 1.98(\mathrm{~s}, 3 \mathrm{H}), 1.25(\mathrm{~s}, 6 \mathrm{H}) .{ }^{13} \mathrm{C} \mathrm{NMR}$ $\left(126 \mathrm{MHz} \mathrm{CDCl}_{3}\right) \delta 168.1,163.8,77.5,72.6,40.8,22.8,11.0$. HRMS (ESI-TOF) Calcd for $\mathrm{C}_{15} \mathrm{H}_{13} \mathrm{~F}_{7} \mathrm{~N}_{3} \mathrm{O}_{5}{ }^{-}[\mathrm{M}-\mathrm{H}]^{-:}$: 448.0743, found: 448.0748 .<smiles>CC(=O)C(C)(C)Cc1ccccc1NC(=O)COc1c(F)c(F)c(F)c(F)c1F</smiles>

(E)-2-benzyl-2-methyl-3-((2-oxo-2-((2,3,5,6-tetrafluoro-4-(trifluoromethyl)phenyl)a mino)ethoxy)imino)butyl nitrate (2b)

Substrate $\mathbf{1 b}$ was nitrooxylated following the general nitrooxylation procedure. After purification by preparative thin-layer chromatography (Hexane $:$ EtOAc $=6: 1$ as eluent), 2b was obtained as a white solid (18.4 mg, 70\%), m.p. $93{ }^{\circ} \mathrm{C} .{ }^{1} \mathrm{H}$ NMR (500 MHz, $\left.\mathrm{CDCl}_{3}\right) \delta 7.46(\mathrm{~s}, 1 \mathrm{H}), 7.18(\mathrm{t}, J=7.7 \mathrm{~Hz}, 2 \mathrm{H}), 7.03(\mathrm{~d}, J=7.2 \mathrm{~Hz}, 2 \mathrm{H}), 6.99(\mathrm{t}, J=7.4$ $\mathrm{Hz}, 1 \mathrm{H}), 4.63(\mathrm{~s}, 2 \mathrm{H}), 4.50(\mathrm{~d}, J=10.6 \mathrm{~Hz}, 1 \mathrm{H}), 4.40(\mathrm{~d}, J=10.6 \mathrm{~Hz}, 1 \mathrm{H}), 2.89(\mathrm{~s}, 2 \mathrm{H})$, 2.07 (s, 3H), 1.29 (s, 3H). ${ }^{13} \mathrm{C}$ NMR $\left(126 \mathrm{MHz}, \mathrm{CDCl}_{3}\right) \delta 168.2,162.8,135.6,130.0$, 128.3, 126.8, 75.0, 72.5, 44.8, 41.5, 20.9, 11.9. HRMS (ESI-TOF) Calcd for $\mathrm{C}_{21} \mathrm{H}_{17} \mathrm{~F}_{7} \mathrm{~N}_{3} \mathrm{O}_{5}^{-}[\mathrm{M}-\mathrm{H}]^{-}:$524.1057, found: 524.1068. The structure of $\mathbf{2 b}$ was also confirmed by X-ray crystallography (CCDC 2076322).

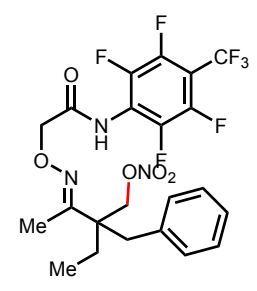

(E)-2-benzyl-2-ethyl-3-((2-0xo-2-((2,3,5,6-tetrafluoro-4-(trifluoromethyl)phenyl)a mino)ethoxy)imino)butyl nitrate (2c)

Substrate 1c was nitrooxylated following the general nitrooxylation procedure. After purification by preparative thin-layer chromatography (Hexane : EtOAc $=6: 1$ as eluent), 2c was obtained as a white solid (20.7 mg, 77\%), m.p. $112{ }^{\circ} \mathrm{C} .{ }^{1} \mathrm{H}$ NMR (500 MHz, $\left.\mathrm{CDCl}_{3}\right) \delta 7.15(\mathrm{t}, J=7.7 \mathrm{~Hz}, 2 \mathrm{H}), 7.00(\mathrm{~d}, J=7.3 \mathrm{~Hz}, 2 \mathrm{H}), 6.91(\mathrm{t}, J=7.4 \mathrm{~Hz}, 1 \mathrm{H})$, $4.63(\mathrm{~d}, J=1.9 \mathrm{~Hz}, 2 \mathrm{H}), 4.48(\mathrm{~s}, 2 \mathrm{H}), 2.89(\mathrm{~d}, J=5.2 \mathrm{~Hz}, 2 \mathrm{H}), 2.09(\mathrm{~s}, 3 \mathrm{H}), 1.80-1.67$ $(\mathrm{m}, 2 \mathrm{H}), 1.30(\mathrm{dd}, J=21.5,7.7 \mathrm{~Hz}, 2 \mathrm{H}), 0.88$ (t, $J=7.5 \mathrm{~Hz}, 3 \mathrm{H}) .{ }^{13} \mathrm{C}$ NMR $(126 \mathrm{MHz}$, $\left.\mathrm{CDCl}_{3}\right) \delta 168.2,162.2,135.9,129.8,128.3,126.7,72.5,70.6,48.6,39.9,26.1,11.7$, 8.3. HRMS (ESI-TOF) Calcd for $\mathrm{C}_{22} \mathrm{H}_{21} \mathrm{~F}_{7} \mathrm{~N}_{3} \mathrm{O}_{5}{ }^{+}[\mathrm{M}+\mathrm{H}]^{+}$: 540.1369, found: 540.1357. 
<smiles>CC(=O)C(C)(C)CCc1ccccc1</smiles>

(E)-2-methyl-3-((2-oxo-2-((2,3,5,6-tetrafluoro-4-(trifluoromethyl)phenyl)amino)e thoxy)imino)-2-phenethylbutyl nitrate (2d)

Substrate 1d was nitrooxylated following the general nitrooxylation procedure. After purification by preparative thin-layer chromatography (Hexane $:$ EtOAc $=6: 1$ as eluent), 2d was obtained as a white solid (18.3 mg, 68\%), m.p. $49{ }^{\circ} \mathrm{C} .{ }^{1} \mathrm{H}$ NMR (500 MHz, $\left.\mathrm{CDCl}_{3}\right) \delta 7.81(\mathrm{~s}, 1 \mathrm{H}), 7.28(\mathrm{~d}, J=7.6 \mathrm{~Hz}, 2 \mathrm{H}), 7.20(\mathrm{~s}, 1 \mathrm{H}), 7.13(\mathrm{~d}, J=7.1 \mathrm{~Hz}, 2 \mathrm{H})$, $4.76(\mathrm{~s}, 2 \mathrm{H}), 4.57(\mathrm{~d}, J=19.3 \mathrm{~Hz}, 2 \mathrm{H}), 2.51(\mathrm{~s}, 2 \mathrm{H}), 1.98(\mathrm{~s}, 3 \mathrm{H}), 1.89-1.70(\mathrm{~m}, 2 \mathrm{H})$, $1.31(\mathrm{~s}, 3 \mathrm{H}) .{ }^{13} \mathrm{C} \mathrm{NMR}\left(126 \mathrm{MHz}, \mathrm{CDCl}_{3}\right) \delta 167.9,162.9,140.8,128.7,128.2,126.4$, 75.9, 72.8, 44.1, 37.4, 30.4, 21.0, 11.2. HRMS (ESI-TOF) Calcd for $\mathrm{C}_{22} \mathrm{H}_{19} \mathrm{~F}_{7} \mathrm{~N}_{3} \mathrm{O}_{5}{ }^{-}$[M$\mathrm{H}]:$ : 538.1213, found: 538.1230 .<smiles>CCC(CO)(CCc1ccccc1)C(C)=NOCC(=O)Nc1c(F)c(F)c(C(F)(F)F)c(F)c1F</smiles>

(E)-2-ethyl-3-((2-oxo-2-((2,3,5,6-tetrafluoro-4-(trifluoromethyl)phenyl)amino)eth oxy)imino)-2-phenethylbutyl nitrate (2e)

Substrate 1e was nitrooxylated following the general nitrooxylation procedure, but $\mathrm{Al}\left(\mathrm{NO}_{3}\right)_{3} \cdot 9 \mathrm{H}_{2} \mathrm{O}$ was used instead of $\mathrm{Fe}\left(\mathrm{NO}_{3}\right)_{3} \cdot 9 \mathrm{H}_{2} \mathrm{O}$. After purification by preparative thin-layer chromatography (Hexane : EtOAc $=6: 1$ as eluent), 2e was obtained as a white solid (20.2 mg, 73\%), m.p. $64{ }^{\circ} \mathrm{C} .{ }^{1} \mathrm{H} \mathrm{NMR}\left(500 \mathrm{MHz}, \mathrm{CDCl}_{3}\right) \delta 7.76(\mathrm{~s}, 1 \mathrm{H})$, $7.28(\mathrm{~d}, J=7.7 \mathrm{~Hz}, 2 \mathrm{H}), 7.21(\mathrm{t}, J=7.2 \mathrm{~Hz}, 1 \mathrm{H}), 7.13(\mathrm{~d}, J=7.4 \mathrm{~Hz}, 2 \mathrm{H}), 4.77(\mathrm{~s}, 2 \mathrm{H})$, $4.67(\mathrm{~d}, J=10.5 \mathrm{~Hz}, 2 \mathrm{H}), 2.62-2.53(\mathrm{~m}, 1 \mathrm{H}), 2.43-2.35(\mathrm{~m}, 1 \mathrm{H}), 1.96(\mathrm{~s}, 3 \mathrm{H}), 1.78$ (ddd, $J=22.1,18.2,8.6 \mathrm{~Hz}, 2 \mathrm{H}), 1.61$ (dt, $J=22.0,7.4 \mathrm{~Hz}, 2 \mathrm{H}), 0.85$ (t, $J=7.5 \mathrm{~Hz}$, $3 \mathrm{H}) .{ }^{13} \mathrm{C} \mathrm{NMR}\left(126 \mathrm{MHz}, \mathrm{CDCl}_{3}\right) \delta 167.9,162.6,140.9,128.6,128.2,126.3,72.8,71.4$, 47.5, 35.5, 30.4, 26.4, 10.9, 8.0. HRMS (ESI-TOF) Calcd for $\mathrm{C}_{23} \mathrm{H}_{21} \mathrm{~F}_{7} \mathrm{~N}_{3} \mathrm{O}_{5}^{-}[\mathrm{M}-\mathrm{H}]^{-}$: 552.1370, found: 552.1386 .

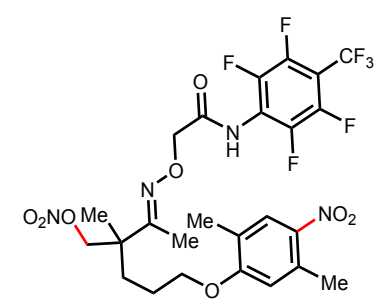

(E)-5-(2,5-dimethyl-4-nitrophenoxy)-2-methyl-2-(1-((2-oxo-2-((perfluorophenyl)a mino)ethoxy)imino)ethyl)pentyl nitrate (2f) 
Substrate 1f was nitrooxylated following the general nitrooxylation procedure. After purification by preparative thin-layer chromatography (Hexane $:$ EtOAc $=6: 1$ as eluent), 2f was obtained as a yellow solid (5.9 mg, 20\%) , m.p. $71{ }^{\circ} \mathrm{C} .{ }^{1} \mathrm{H}$ NMR $(500 \mathrm{MHz}$, $\left.\mathrm{CDCl}_{3}\right) \delta 7.92(\mathrm{~s}, 1 \mathrm{H}), 7.77(\mathrm{~s}, 1 \mathrm{H}), 6.61(\mathrm{~s}, 1 \mathrm{H}), 4.75(\mathrm{~d}, J=3.3 \mathrm{~Hz}, 2 \mathrm{H}), 4.56(\mathrm{~d}, J=$ $9.4 \mathrm{~Hz}, 2 \mathrm{H}), 4.05-3.98(\mathrm{~m}, 2 \mathrm{H}), 2.61(\mathrm{~s}, 3 \mathrm{H}), 2.20(\mathrm{~s}, 3 \mathrm{H}), 2.00(\mathrm{~d}, J=5.6 \mathrm{~Hz}, 3 \mathrm{H})$, $1.77-1.74(\mathrm{~m}, 2 \mathrm{H}), 1.43(\mathrm{~d}, J=5.6 \mathrm{~Hz}, 2 \mathrm{H}), 1.29(\mathrm{~s}, 3 \mathrm{H}) .{ }^{13} \mathrm{C} \mathrm{NMR}(126 \mathrm{MHz}$, $\left.\mathrm{CD}_{3} \mathrm{OD} \_\mathrm{SPE}\right) \delta 169.7,161.5,160.6,141.2,134.3,126.8,125.2,113.6,76.0,72.1,68.1$, 43.3, 31.2, 23.5, 20.0, 14.3, 9.6. HRMS (ESI-TOF) Calcd for $\mathrm{C}_{25} \mathrm{H}_{26} \mathrm{~F}_{7} \mathrm{~N}_{4} \mathrm{O}_{8}{ }^{+}[\mathrm{M}+\mathrm{H}]^{+}$: 643.1639, found: 643.1628 .

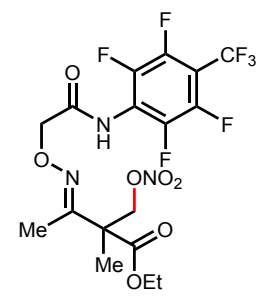

(E)-2-methyl-2-((nitrooxy)methyl)-3-((2-0xo-2-((2,3,5,6-tetrafluoro-4-(trifluorom ethyl)phenyl)amino)ethoxy)imino)butanoate ethyl (2g)

Substrate 1g was nitrooxylated following the general nitrooxylation procedure. After purification by preparative thin-layer chromatography (Hexane $:$ EtOAc $=6: 1$ as eluent), 2 g was obtained as a white oil $(16.0 \mathrm{mg}, 63 \%) .{ }^{1} \mathrm{H} \mathrm{NMR}\left(500 \mathrm{MHz}, \mathrm{CDCl}_{3}\right) \delta 7.77$ (s, 1H), 4.84 (dd, $J=18.9,10.3 \mathrm{~Hz}, 2 \mathrm{H}), 4.77$ (s, 2H), 4.24 (dd, $J=7.1,2.0 \mathrm{~Hz}, 2 \mathrm{H}), 1.98$ $(\mathrm{s}, 3 \mathrm{H}), 1.51(\mathrm{~s}, 3 \mathrm{H}), 1.28(\mathrm{~d}, J=7.1 \mathrm{~Hz}, 3 \mathrm{H}) .{ }^{13} \mathrm{C} \mathrm{NMR}\left(126 \mathrm{MHz}, \mathrm{CDCl}_{3}\right) \delta 170.5$, 167.7, 159.3, 73.9, 73.0, 62.4, 52.3, 18.7, 14.0, 12.7. HRMS (ESI-TOF) Calcd for $\mathrm{C}_{17} \mathrm{H}_{15} \mathrm{~F}_{7} \mathrm{~N}_{3} \mathrm{O}_{7}^{-}[\mathrm{M}-\mathrm{H}]^{-}:$506.0798, found: 506.0810.<smiles>CC(C)(C)C(C)(C)/C(=N\OCC(=O)Nc1c(O)c(F)c(F)c(F)c1F)c1ccccc1</smiles>

(E)-2,2-dimethyl-3-((2-oxo-2-((2,3,5,6-tetrafluoro-4-(trifluoromethyl)phenyl)amin o)ethoxy)imino)-3-phenylpropyl nitrate (2h)

Substrate $1 \mathbf{h}$ was nitrooxylated following the general nitrooxylation procedure. After purification by preparative thin-layer chromatography (Hexane : EtOAc $=6: 1$ as eluent), 2h was obtained as a white solid (19.7 mg, 77\%), m.p. $74{ }^{\circ} \mathrm{C} .{ }^{1} \mathrm{H}$ NMR (500 MHz, $\left.\mathrm{CDCl}_{3}\right) \delta 7.55(\mathrm{~s}, 1 \mathrm{H}), 7.48(\mathrm{~s}, 1 \mathrm{H}), 7.45(\mathrm{dd}, J=7.3,3.2 \mathrm{~Hz}, 2 \mathrm{H}), 7.13-7.08(\mathrm{~m}, 2 \mathrm{H})$, 4.69 (s, 2H), 4.47 (s, 2H), 1.29 (s, 6H). ${ }^{13} \mathrm{C} \mathrm{NMR}\left(126 \mathrm{MHz}, \mathrm{CDCl}_{3}\right) \delta 167.4,165.8$, 131.9, 129.1, 128.9, 126.8, 77.5, 72.9, 41.0, 23.4. HRMS (ESI-TOF) Calcd for $\mathrm{C}_{20} \mathrm{H}_{15} \mathrm{~F}_{7} \mathrm{~N}_{3} \mathrm{O}_{5}^{-}[\mathrm{M}-\mathrm{H}]^{-:}$: 510.0900, found: 510.0912 . 
<smiles>COc1c(F)c(C(F)(F)F)c(F)c(F)c1NC(=O)CO/N=C(\c1ccc(C)cc1)C(C)(C)C[N+](=O)[O-]</smiles>

(E)-2,2-dimethyl-3-((2-oxo-2-((2,3,5,6-tetrafluoro-4-(trifluoromethyl)phenyl)amin o)ethoxy)imino)-3-(p-tolyl)propyl nitrate (2i)

Substrate 1i was nitrooxylated following the general nitrooxylation procedure. After purification by preparative thin-layer chromatography (Hexane $:$ EtOAc $=6: 1$ as eluent), $2 \mathbf{i}$ was obtained as a white solid $(13.4 \mathrm{mg}, 51 \%)$, m.p. $75^{\circ} \mathrm{C} .{ }^{1} \mathrm{H}$ NMR $\left(500 \mathrm{MHz}, \mathrm{CDCl}_{3}\right)$ $\delta 7.58(\mathrm{~s}, 1 \mathrm{H}), 7.28(\mathrm{~d}, J=7.9 \mathrm{~Hz}, 2 \mathrm{H}), 6.99(\mathrm{~d}, J=8.0 \mathrm{~Hz}, 2 \mathrm{H}), 4.68(\mathrm{~s}, 2 \mathrm{H}), 4.45$ (s, 2H), $2.40(\mathrm{~s}, 3 \mathrm{H}), 1.27(\mathrm{~s}, 6 \mathrm{H}) .{ }^{13} \mathrm{C}$ NMR $\left(126 \mathrm{MHz}, \mathrm{CDCl}_{3}\right) \delta 167.5,166.0,139.2$, 129.6, 128.8, 126.6, 77.5, 72.9, 41.0, 23.4, 21.3. HRMS (ESI-TOF) Calcd for $\mathrm{C}_{21} \mathrm{H}_{17} \mathrm{~F}_{7} \mathrm{~N}_{3} \mathrm{O}_{5}{ }^{-}[\mathrm{M}-\mathrm{H}]^{-}:$:524.1057, found: 524.1071.<smiles>COc1ccc(C(=NOCC(=O)Nc2c(F)c(F)c(F)c(F)c2F)C(C)(C)C)cc1</smiles>

(E)-3-(4-methoxyphenyl)-2,2-dimethyl-3-((2-oxo-2-((2,3,5,6-tetrafluoro-4-(trifluo romethyl)phenyl)amino)ethoxy)imino)propyl nitrate $(2 \mathrm{j})$

Substrate $1 \mathbf{j}$ was nitrooxylated following the general nitrooxylation procedure. After purification by preparative thin-layer chromatography (Hexane $:$ EtOAc $=6: 1$ as eluent), $\mathbf{2} \mathbf{j}$ was obtained as a yellow oil $(15.1 \mathrm{mg}, 56 \%) .{ }^{1} \mathrm{H} \mathrm{NMR}\left(500 \mathrm{MHz}, \mathrm{CDCl}_{3}\right) \delta 7.58(\mathrm{~s}$, $1 \mathrm{H}), 7.03(\mathrm{dd}, J=13.6,6.8 \mathrm{~Hz}, 2 \mathrm{H}), 6.99(\mathrm{~d}, J=8.8 \mathrm{~Hz}, 2 \mathrm{H}), 4.68(\mathrm{~s}, 2 \mathrm{H}), 4.45(\mathrm{~s}, 2 \mathrm{H})$, $3.84(\mathrm{~s}, 3 \mathrm{H}), 1.27(\mathrm{~s}, 6 \mathrm{H}) .{ }^{13} \mathrm{C} \mathrm{NMR}\left(126 \mathrm{MHz}, \mathrm{CDCl}_{3}\right) \delta 167.5,165.8,160.0,128.2$, 123.7, 114.3, 77.6, 72.9, 55.3, 41.1, 23.5. HRMS (ESI-TOF) Calcd for $\mathrm{C}_{21} \mathrm{H}_{17} \mathrm{~F}_{7} \mathrm{~N}_{3} \mathrm{O}_{6}{ }^{-}$ [M-H]: :540.1006, found: 540.1020 .

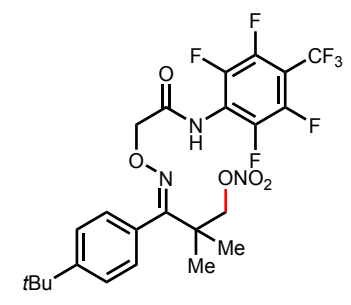

(E)-3-(4-(tert-butyl)phenyl)-2,2-dimethyl-3-((2-oxo-2-((2,3,5,6-tetrafluoro-4-(trifl uoromethyl)phenyl)amino)ethoxy)imino)propyl nitrate (2k)

Substrate 1k was nitrooxylated following the general nitrooxylation procedure. After purification by preparative thin-layer chromatography (Hexane $:$ EtOAc $=6: 1$ as eluent), 2k was obtained as a white solid (15.6 mg, 55\%), m.p. $58{ }^{\circ} \mathrm{C} .{ }^{1} \mathrm{H}$ NMR (500 MHz, $\left.\mathrm{CDCl}_{3}\right) \delta 7.58(\mathrm{~s}, 1 \mathrm{H}), 7.47(\mathrm{~d}, J=8.3 \mathrm{~Hz}, 2 \mathrm{H}), 7.02(\mathrm{~d}, J=8.3 \mathrm{~Hz}, 2 \mathrm{H}), 4.69(\mathrm{~s}, 2 \mathrm{H})$, 
$4.46(\mathrm{~s}, 2 \mathrm{H}), 1.34(\mathrm{~s}, 9 \mathrm{H}), 1.28(\mathrm{~s}, 6 \mathrm{H}) .{ }^{13} \mathrm{C} \mathrm{NMR}\left(126 \mathrm{MHz}, \mathrm{CDCl}_{3}\right) \delta$ 167.5, 166.0, 152.2, 128.8, 126.4, 125.8, 77.6, 72.9, 41.0, 34.8, 31.2, 23.4. HRMS (ESI-TOF) Calcd for $\mathrm{C}_{24} \mathrm{H}_{23} \mathrm{~F}_{7} \mathrm{~N}_{3} \mathrm{O}_{5}{ }^{-}[\mathrm{M}-\mathrm{H}]^{-}: 566.1526$, found: 566.1543 .<smiles>CC(C)(C)C(C)(C)C(=NOCC(=O)Nc1c(F)c(F)c(F)c(F)c1F)c1ccc(Cl)cc1</smiles>

(E)-3-(4-chlorophenyl)-2,2-dimethyl-3-((2-oxo-2-((2,3,5,6-tetrafluoro-4-(trifluoro methyl)phenyl)amino)ethoxy)imino)propyl nitrate (2I)

Substrate 11 was nitrooxylated following the general nitrooxylation procedure. After purification by preparative thin-layer chromatography (Hexane $:$ EtOAc $=6: 1$ as eluent), $2 \mathrm{l}$ was obtained as a white solid $(15.3 \mathrm{mg}, 56 \%)$, m.p. $69{ }^{\circ} \mathrm{C} .{ }^{1} \mathrm{H}$ NMR $(500 \mathrm{MHz}$, $\left.\mathrm{CDCl}_{3}\right) \delta 7.50(\mathrm{~s}, 1 \mathrm{H}), 7.47(\mathrm{~d}, J=8.3 \mathrm{~Hz}, 2 \mathrm{H}), 7.06(\mathrm{~d}, J=8.4 \mathrm{~Hz}, 2 \mathrm{H}), 4.69(\mathrm{~s}, 2 \mathrm{H})$, $4.46(\mathrm{~s}, 2 \mathrm{H}), 1.27(\mathrm{~s}, 6 \mathrm{H}) .{ }^{13} \mathrm{C} \mathrm{NMR}\left(126 \mathrm{MHz}, \mathrm{CDCl}_{3}\right) \delta 167.1,164.9,135.4,130.1$, 129.3, 128.3, 73.0, 41.0, 23.5, 14.2. HRMS (ESI-TOF) Calcd for $\mathrm{C}_{20} \mathrm{H}_{14} \mathrm{ClF}_{7} \mathrm{~N}_{3} \mathrm{O}_{5}{ }^{-}$[MH]: 544.0510, found: 544.0520 .<smiles>CC(C)(C)C(=NOCC(=O)Nc1c(O)c(F)c(F)c(F)c1F)c1ccc(F)cc1</smiles>

(E)-3-(4-fluorophenyl)-2,2-dimethyl-3-((2-oxo-2-((2,3,5,6-tetrafluoro-4-(trifluoro methyl)phenyl)amino)ethoxy)imino)propyl nitrate (2m)

Substrate 1m was nitrooxylated following the general nitrooxylation procedure. After purification by preparative thin-layer chromatography (Hexane : EtOAc $=6: 1$ as eluent), 2m was obtained as a white solid (15.6 mg, 59\%), m.p. $77{ }^{\circ} \mathrm{C} .{ }^{1} \mathrm{H}$ NMR $(500 \mathrm{MHz}$, $\left.\mathrm{CDCl}_{3}\right) \delta 7.52(\mathrm{~s}, 1 \mathrm{H}), 7.18(\mathrm{t}, J=8.5 \mathrm{~Hz}, 2 \mathrm{H}), 7.12-7.07(\mathrm{~m}, 2 \mathrm{H}), 4.69(\mathrm{~s}, 2 \mathrm{H}), 4.46$ $(\mathrm{s}, 2 \mathrm{H}), 1.28(\mathrm{~s}, 6 \mathrm{H}) .{ }^{13} \mathrm{C} \mathrm{NMR}\left(126 \mathrm{MHz}, \mathrm{CDCl}_{3}\right) \delta 167.2,165.0,163.9,161.9,128.8$, 128.8, 127.6, 127.6, 116.2, 116.1, 77.4, 73.0, 41.0, 23.5. HRMS (ESI-TOF) Calcd for $\mathrm{C}_{20} \mathrm{H}_{14} \mathrm{~F}_{8} \mathrm{~N}_{3} \mathrm{O}_{5}^{-}[\mathrm{M}-\mathrm{H}]:: 528.0806$, found: 528.0809 .<smiles>COc1cccc(C(=NOCC(=O)Nc2c(F)c(F)c(F)c(F)c2F)C(C)(C)C)c1</smiles>

(E)-3-(3-methoxyphenyl)-2,2-dimethyl-3-((2-oxo-2-((2,3,5,6-tetrafluoro-4-(trifluo romethyl)phenyl)amino)ethoxy)imino)propyl nitrate (2n) 
Substrate 1n was nitrooxylated following the general nitrooxylation procedure. After purification by preparative thin-layer chromatography (Hexane $:$ EtOAc $=6: 1$ as eluent), 2n was obtained as a white solid (16.2 mg, 60\%), m.p. $56{ }^{\circ} \mathrm{C} .{ }^{1} \mathrm{H}$ NMR (500 MHz, $\left.\mathrm{CDCl}_{3}\right) \delta 7.57(\mathrm{~s}, 1 \mathrm{H}), 7.39(\mathrm{t}, J=7.9 \mathrm{~Hz}, 1 \mathrm{H}), 6.96(\mathrm{dd}, J=8.4,2.4 \mathrm{~Hz}, 1 \mathrm{H}), 6.68-$ $6.60(\mathrm{~m}, 2 \mathrm{H}), 4.69(\mathrm{~s}, 2 \mathrm{H}), 4.46(\mathrm{~s}, 2 \mathrm{H}), 3.82(\mathrm{~s}, 3 \mathrm{H}), 1.29(\mathrm{~s}, 6 \mathrm{H}) .{ }^{13} \mathrm{C}$ NMR $(126 \mathrm{MHz}$, $\left.\mathrm{CDCl}_{3}\right) \delta 167.4,165.6,159.8,133.1,130.1,118.7,113.9,113.1,77.5,77.3,77.1,76.8$, 73.0, 55.3, 40.9, 23.5. HRMS (ESI-TOF) Calcd for $\mathrm{C}_{21} \mathrm{H}_{17} \mathrm{~F}_{7} \mathrm{~N}_{3} \mathrm{O}_{6}{ }^{-}[\mathrm{M}-\mathrm{H}]$ : : 540.1006, found: 540.1019 .<smiles>CC(C)(C)C(Cc1ccccc1)=NOCC(=O)Nc1c(O)c(F)c(F)c(F)c1F</smiles>

(E)-2,2-dimethyl-3-((2-oxo-2-((2,3,5,6-tetrafluoro-4-(trifluoromethyl)phenyl)amin o)ethoxy)imino)-4-phenylbutyl nitrate (20)

Substrate 10 was nitrooxylated following the general nitrooxylation procedure. After purification by preparative thin-layer chromatography (Hexane : EtOAc $=6: 1$ as eluent), 20 was obtained as a white solid (18.9 mg, 72\%), m.p. $85{ }^{\circ} \mathrm{C} .{ }^{1} \mathrm{H}$ NMR (400 MHz, $\left.\mathrm{CDCl}_{3}\right) \delta 7.29(\mathrm{~d}, J=1.6 \mathrm{~Hz}, 2 \mathrm{H}), 7.23(\mathrm{~d}, J=7.1 \mathrm{~Hz}, 2 \mathrm{H}), 7.19-7.15(\mathrm{~m}, 1 \mathrm{H}), 4.74$ $(\mathrm{s}, 2 \mathrm{H}), 4.54(\mathrm{~s}, 2 \mathrm{H}), 3.84(\mathrm{~s}, 2 \mathrm{H}), 1.32(\mathrm{~s}, 6 \mathrm{H}) .{ }^{13} \mathrm{C} \mathrm{NMR}\left(126 \mathrm{MHz}, \mathrm{CDCl}_{3}\right) \delta 167.7$, 164.1, 136.1, 129.0, 127.9, 126.8, 77.7, 73.0, 41.1, 32.2, 23.4. HRMS (ESI-TOF) Calcd for $\mathrm{C}_{21} \mathrm{H}_{17} \mathrm{~F}_{7} \mathrm{~N}_{3} \mathrm{O}_{5}{ }^{-}[\mathrm{M}-\mathrm{H}]$ : : 524.1057, found: 524.1071.<smiles>CC(C)(C)[C@@H](O)CC(=O)OCC(=O)Nc1c(F)c(F)c(F)c(F)c1F</smiles>

(E)-4,4-dimethyl-5-(nitrooxy)-3-((2-oxo-2-((2,3,5,6-tetrafluoro-4-(trifluoromethyl)p henyl)amino)ethoxy)imino)pentanoate ethyl (2p)

Substrate 1pwas nitrooxylated following the general nitrooxylation procedure. After purification by preparative thin-layer chromatography (Hexane : EtOAc $=6: 1$ as eluent), 2p was obtained as a yellow oil (18.5 mg, 71\%). ${ }^{1} \mathrm{H}$ NMR $\left(500 \mathrm{MHz}, \mathrm{CDCl}_{3}\right) \delta 9.12$ (s, 1H), 4.78 (s, 2H), 4.47 (s, 2H), 4.19 (d, J=7.1 Hz, 2H), 3.50 (s, 2H), 1.23 (s, 6H). ${ }^{13} \mathrm{C}$ NMR $\left(126 \mathrm{MHz}, \mathrm{CDCl}_{3}\right) \delta 169.8,168.7,158.4,77.6,73.1,62.5,40.6,31.6,22.4$, 14.0. HRMS (ESI-TOF) Calcd for $\mathrm{C}_{18} \mathrm{H}_{17} \mathrm{~F}_{7} \mathrm{~N}_{3} \mathrm{O}_{7}{ }^{-}[\mathrm{M}-\mathrm{H}]^{-:}$: 520.0955, found: 520.0966. 
<smiles>CCC/C(=N/OCC(=O)Nc1c(F)c(F)c(F)c(F)c1F)C(C)(C)C</smiles>

(E)-2,2-dimethyl-3-((2-oxo-2-((2,3,5,6-tetrafluoro-4-(trifluoromethyl)phenyl)amin o)ethoxy)imino)hexyl nitrate (2q)

Substrate 1q was nitrooxylated following the general nitrooxylation procedure. After purification by preparative thin-layer chromatography (Hexane $:$ EtOAc $=6: 1$ as eluent), 2q was obtained as a yellow solid (16.4 mg, 69\%), m.p. $65{ }^{\circ} \mathrm{C} .{ }^{1} \mathrm{H}$ NMR (500 MHz, $\left.\mathrm{CDCl}_{3}\right) \delta 7.85(\mathrm{~s}, 1 \mathrm{H}), 4.71(\mathrm{~s}, 2 \mathrm{H}), 4.49(\mathrm{~s}, 2 \mathrm{H}), 2.35-2.30(\mathrm{~m}, 2 \mathrm{H}), 1.60(\mathrm{~d}, J=4.1$ $\mathrm{Hz}, 2 \mathrm{H}), 1.25(\mathrm{~s}, 6 \mathrm{H}), 1.02(\mathrm{t}, J=7.3 \mathrm{~Hz}, 3 \mathrm{H}) .{ }^{13} \mathrm{C} \mathrm{NMR}\left(126 \mathrm{MHz}, \mathrm{CDCl}_{3}\right) \delta 168.1$, 167.1, 77.6, 72.7, 41.0, 28.7, 23.0, 20.3, 14.8. HRMS (ESI-TOF) Calcd for $\mathrm{C}_{17} \mathrm{H}_{18} \mathrm{~F}_{7} \mathrm{~N}_{3} \mathrm{O}_{5}{ }^{-}[\mathrm{M}-\mathrm{H}]^{-}:$: 476.1057, found: 476.1067 .

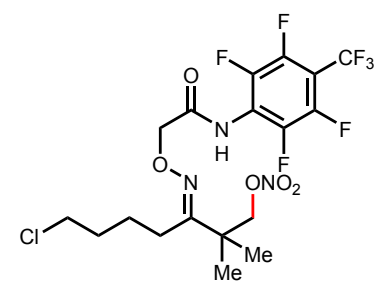

(E)-7-chloro-2,2-dimethyl-3-((2-oxo-2-((2,3,5,6-tetrafluoro-4-(trifluoromethyl)ph enyl)amino)ethoxy)imino)heptyl nitrate (2r)

Substrate 1r was nitrooxylated following the general nitrooxylation procedure. After purification by preparative thin-layer chromatography (Hexane $:$ EtOAc $=6: 1$ as eluent), 2r was obtained as a yellow solid (16.0 mg, 61\%), m.p. $62{ }^{\circ} \mathrm{C} .{ }^{1} \mathrm{H}$ NMR (500 MHz, $\left.\mathrm{CDCl}_{3}\right) \delta 7.84(\mathrm{~s}, 1 \mathrm{H}), 4.72(\mathrm{~s}, 2 \mathrm{H}), 4.49(\mathrm{~s}, 2 \mathrm{H}), 3.59(\mathrm{t}, J=6.2 \mathrm{~Hz}, 2 \mathrm{H}), 2.40-2.34$ $(\mathrm{m}, 2 \mathrm{H}), 1.91-1.83(\mathrm{~m}, 2 \mathrm{H}), 1.79-1.70(\mathrm{~m}, 2 \mathrm{H}), 1.26(\mathrm{~s}, 6 \mathrm{H}) .{ }^{13} \mathrm{C}$ NMR $(126 \mathrm{MHz}$, $\left.\mathrm{CDCl}_{3}\right) \delta 167.9,166.5,77.5,72.8,44.2,41.0,32.6,25.7,23.7,23.0$. HRMS (ESI-TOF) Calcd for $\mathrm{C}_{18} \mathrm{H}_{18} \mathrm{ClF}_{7} \mathrm{~N}_{3} \mathrm{O}_{5}{ }^{-}$[M-H]-: 524.0823, found: 524.0839.

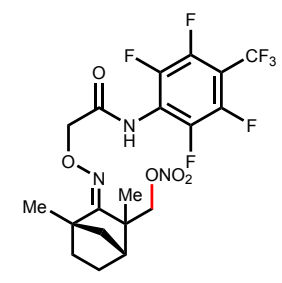

((1R,4S,E)-2,4-dimethyl-3-((2-oxo-2-((2,3,5,6-tetrafluoro-4-(trifluoromethyl)phen yl)amino)ethoxy)imino)bicyclo[2.2.1] heptan-2-yl)methyl nitrate (2s)

Substrate 1s was nitrooxylated following the general nitrooxylation procedure, but $\mathrm{Al}\left(\mathrm{NO}_{3}\right)_{3} \cdot 9 \mathrm{H}_{2} \mathrm{O}$ was used instead of $\mathrm{Fe}\left(\mathrm{NO}_{3}\right)_{3} \cdot 9 \mathrm{H}_{2} \mathrm{O}$. After purification by preparative thin-layer chromatography (Hexane $: \mathrm{EtOAc}=6: 1$ as eluent), $2 \mathrm{~s}$ was obtained as a white solid (18.6 mg, 73\%), m.p. $89{ }^{\circ} \mathrm{C} .{ }^{1} \mathrm{H}$ NMR $\left(500 \mathrm{MHz}, \mathrm{CDCl}_{3}\right) \delta 7.89(\mathrm{~s}, 1 \mathrm{H}), 4.76$ (d, $J=11.2 \mathrm{~Hz}, 1 \mathrm{H}), 4.65(\mathrm{~s}, 2 \mathrm{H}), 4.63(\mathrm{~s}, 1 \mathrm{H}), 1.86(\mathrm{~s}, 3 \mathrm{H}), 1.65-1.51(\mathrm{~m}, 4 \mathrm{H}), 1.36(\mathrm{~d}$, 
$J=11.5 \mathrm{~Hz}, 6 \mathrm{H}) .{ }^{13} \mathrm{C} \mathrm{NMR}\left(126 \mathrm{MHz}, \mathrm{CDCl}_{3}\right) \delta 173.0,168.2,73.0,71.8,52.8,48.4$, 45.1, 39.1, 29.8, 24.2, 23.2, 22.5. HRMS (ESI-TOF) Calcd for $\mathrm{C}_{19} \mathrm{H}_{17} \mathrm{~F}_{7} \mathrm{~N}_{3} \mathrm{O}_{5}^{-}[\mathrm{M}-\mathrm{H}]^{-}:$ 500.1057, found: 500.1073 .

The Scope of C(sp $\left.{ }^{3}\right)-H$ Nitrooxylation of Amides

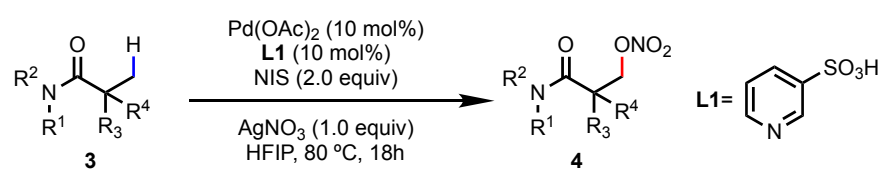

General procedure for nitrooxylation of amide substrates: In the reaction tube, $\mathrm{Pd}(\mathrm{OAc})_{2}(10 \mathrm{~mol} \%, 2.2 \mathrm{mg}), \mathbf{L 1}(10 \mathrm{~mol} \%, 1.6 \mathrm{mg}), \mathrm{NIS}$ (2.0 equiv, $\left.45.0 \mathrm{mg}\right), \mathrm{AgNO}_{3}$ (1.0 equiv, $16.9 \mathrm{mg}$ ), and the amide substrate 3 were weighed and placed with a magnetic stir bar. Then, $1 \mathrm{~mL}$ of HFIP was added to the reaction tube. The reaction mixture was stirred at r.t. for $5 \mathrm{~min}$, and then heated to $80{ }^{\circ} \mathrm{C}$ in oil bath for $18 \mathrm{~h}$. After cooled down to room temperature, the resulting mixture was diluted with DCM, filtered through a plug of Celite and concentrated under reduced pressure. The crude mixture was purified by flash column chromatography to afford the nitrooxylation product. (prep-TLC was used for purification of UV active compounds).<smiles>[M]C(CO[N+](=O)[O-])C(=O)N(C)[Na]</smiles>

\section{3-(dimethylamino)-2-methyl-3-oxopropyl nitrate (4a)}

Substrate 3a was nitrooxylated following the general nitrooxylation procedure. After purification by flash column chromatography, $4 \mathbf{a}$ was obtained as colorless oil (16.2 $\mathrm{mg}, 92 \%)$. For large scale $(5 \mathrm{mmol})$ reaction, 3a $(5.0 \mathrm{mmol}, 575 \mathrm{mg}), \mathrm{Pd}(\mathrm{OAc})_{2}(10$ mol\%, $112 \mathrm{mg}$ ), L1 (10 mol\%, $80 \mathrm{mg}$ ), NIS (2.0 equiv, $2.25 \mathrm{~g}$ ), $\mathrm{AgNO}_{3}$ (1.0 equiv, 849 $\mathrm{mg}$ ) and $50 \mathrm{~mL}$ of HFIP were used. The reaction mixture was stirred at r.t. for $5 \mathrm{~min}$, and then heated to $80{ }^{\circ} \mathrm{C}$ in oil bath for $40 \mathrm{~h}$. After cooled down to room temperature, the resulting mixture was diluted with EA, filtered through a plug of Celite and concentrated under reduced pressure. The crude mixture was purified by flash column chromatography to afford $683 \mathrm{mg}(78 \%)$ of $4 a .{ }^{1} \mathrm{H}$ NMR $\left(600 \mathrm{MHz}, \mathrm{CDCl}_{3}\right) \delta 4.79$ (dd, $J=10.5,8.0 \mathrm{~Hz}, 1 \mathrm{H}), 4.40(\mathrm{dd}, J=10.5,5.9 \mathrm{~Hz}, 1 \mathrm{H}), 3.14$ (ddt, $J=8.0,7.0,5.9 \mathrm{~Hz}, 1 \mathrm{H})$, $3.07(\mathrm{~s}, 3 \mathrm{H}), 2.98(\mathrm{~s}, 3 \mathrm{H}), 1.21(\mathrm{~d}, J=7.0 \mathrm{~Hz}, 3 \mathrm{H}) .{ }^{13} \mathrm{C} \mathrm{NMR}\left(151 \mathrm{MHz}, \mathrm{CDCl}_{3}\right) \delta$ 172.5, 74.8, 37.3, 35.9, 34.0, 14.5., HRMS (ESI-TOF) Calcd for $\mathrm{C}_{6} \mathrm{H}_{13} \mathrm{~N}_{2} \mathrm{O}_{4}{ }^{+}[\mathrm{M}+\mathrm{H}]^{+}$: 177.0875, found: 177.0871 . 


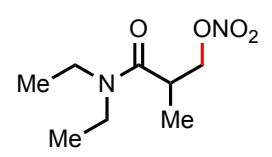

4b

3-(diethylamino)-2-methyl-3-oxopropyl nitrate (4b)

Yellow oil. (16.3 mg, 80\%), ${ }^{1} \mathrm{H}$ NMR $\left(500 \mathrm{MHz}, \mathrm{CDCl}_{3}\right) \delta 4.8(\mathrm{dd}, J=10.5,8.1 \mathrm{~Hz}$, $1 \mathrm{H}), 4.4(\mathrm{dd}, J=10.5,5.9 \mathrm{~Hz}, 1 \mathrm{H}), 3.5(\mathrm{dq}, J=14.1,7.1 \mathrm{~Hz}, 1 \mathrm{H}), 3.4-3.3(\mathrm{~m}, 4 \mathrm{H})$, $3.1-3.0(\mathrm{~m}, 1 \mathrm{H}), \delta 1.2-1.2(\mathrm{~m}, 6 \mathrm{H}), 1.2-1.1(\mathrm{~m}, 3 \mathrm{H}) .{ }^{13} \mathrm{C} \mathrm{NMR}\left(151 \mathrm{MHz}, \mathrm{CDCl}_{3}\right)$ $\delta$ 171.9, 75.1, 42.1, 40.7, 34.0, 15.1, 15.0, 13.2., HRMS (ESI-TOF) Calcd for $\mathrm{C}_{8} \mathrm{H}_{17} \mathrm{~N}_{2} \mathrm{O}_{4}^{+}[\mathrm{M}+\mathrm{H}]^{+}:$205.1188, found: 205.1189 .<smiles>[M]C(CO[N+](=O)[O-])C(=O)N1CCCC1</smiles>

\section{2-methyl-3-oxo-3-(pyrrolidin-1-yl)propyl nitrate (4c)}

Colorless oil. (18.2 mg, 90\%), ${ }^{1} \mathrm{H}$ NMR $\left(600 \mathrm{MHz}, \mathrm{CDCl}_{3}\right) \delta 4.8(\mathrm{dd}, J=10.5,8.3 \mathrm{~Hz}$, $1 \mathrm{H}), 4.4(\mathrm{dd}, J=10.5,5.8 \mathrm{~Hz}, 1 \mathrm{H}), 3.5-3.4(\mathrm{~m}, 4 \mathrm{H}), 3.0(\mathrm{dqd}, J=8.3,7.0,5.8 \mathrm{~Hz}$, $1 \mathrm{H}), 2.1-1.9(\mathrm{~m}, 2 \mathrm{H}), 1.9-1.8(\mathrm{~m}, 2 \mathrm{H}), 1.2(\mathrm{~d}, J=7.0 \mathrm{~Hz}, 3 \mathrm{H}) .{ }^{13} \mathrm{C}$ NMR $(151 \mathrm{MHz}$, $\left.\mathrm{CDCl}_{3}\right) \delta 171.0,74.8,46.6,46.1,36.1,26.2,24.4,14.3$., HRMS (ESI-TOF) Calcd for $\mathrm{C}_{8} \mathrm{H}_{15} \mathrm{~N}_{2} \mathrm{O}_{4}{ }^{+}[\mathrm{M}+\mathrm{H}]^{+}:$203.1032, found: 203.1030 .

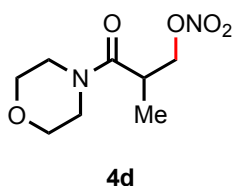

\section{2-methyl-3-morpholino-3-oxopropyl nitrate (4d)}

Colorless oil. (13.5 mg, 62\%), ${ }^{1} \mathrm{H}$ NMR $\left(600 \mathrm{MHz}, \mathrm{CDCl}_{3}\right) \delta 4.8(\mathrm{dd}, J=10.6,8.1 \mathrm{~Hz}$, $1 \mathrm{H}), 4.4(\mathrm{dd}, J=10.6,5.8 \mathrm{~Hz}, 1 \mathrm{H}), 3.8-3.6(\mathrm{~m}, 6 \mathrm{H}), 3.6-3.4(\mathrm{~m}, 2 \mathrm{H}), 3.1(\mathrm{dqd}, J=$ 8.1, 7.0, $5.8 \mathrm{~Hz}, 1 \mathrm{H}), 1.2(\mathrm{~d}, J=7.0 \mathrm{~Hz}, 3 \mathrm{H}) .{ }^{13} \mathrm{C} \mathrm{NMR}\left(151 \mathrm{MHz}, \mathrm{CDCl}_{3}\right) \delta 171.2$, 74.6, 67.0, 66.8, 46.2, 42.5, 33.6, 14.7., HRMS (ESI-TOF) Calcd for $\mathrm{C}_{8} \mathrm{H}_{15} \mathrm{~N}_{2} \mathrm{O}_{5}{ }^{+}$ $[\mathrm{M}+\mathrm{H}]^{+}: 219.0981$, found: 219.0984 .<smiles>CN(C)C(=O)CCO[N+](=O)[O-]</smiles>

\section{3-(dimethylamino)-3-oxopropyl nitrate (4e)}

Colorless oil. (6.0 mg, 37\%), ${ }^{1} \mathrm{H}$ NMR $\left(600 \mathrm{MHz}, \mathrm{CDCl}_{3}\right) \delta 4.8(\mathrm{t}, J=6.7 \mathrm{~Hz}, 2 \mathrm{H}), 3.0$ $(\mathrm{s}, 3 \mathrm{H}), 3.0(\mathrm{~s}, 3 \mathrm{H}), 2.7(\mathrm{t}, J=6.7 \mathrm{~Hz}, 2 \mathrm{H}) .{ }^{13} \mathrm{C} \mathrm{NMR}\left(151 \mathrm{MHz}, \mathrm{CDCl}_{3}\right) \delta 168.5,69.2$, 37.2, 35.6, 30.7., HRMS (ESI-TOF) Calcd for $\mathrm{C}_{5} \mathrm{H}_{11} \mathrm{~N}_{2} \mathrm{O}_{4}{ }^{+}[\mathrm{M}+\mathrm{H}]^{+}$: 163.0714, found: 163.0717 . 


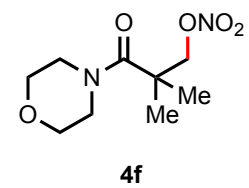

2,2-dimethyl-3-morpholino-3-oxopropyl nitrate (4f)

White solid. (16.9 mg, 73\%), m.p. $62{ }^{\circ} \mathrm{C},{ }^{1} \mathrm{H}$ NMR $\left(600 \mathrm{MHz}, \mathrm{CDCl}_{3}\right) \delta 4.6$ (s, 2H), 3.7 - $3.6(\mathrm{~m}, 8 \mathrm{H}), 1.4(\mathrm{~s}, 6 \mathrm{H}) .{ }^{13} \mathrm{C} \mathrm{NMR}\left(151 \mathrm{MHz}, \mathrm{CDCl}_{3}\right) \delta 173.0,79.3,66.9,45.7,42.2$, 22.4., HRMS (ESI-TOF) Calcd for $\mathrm{C}_{9} \mathrm{H}_{17} \mathrm{~N}_{2} \mathrm{O}_{5}{ }^{+}[\mathrm{M}+\mathrm{H}]^{+}:$: 233.1137, found: 233.1135 .

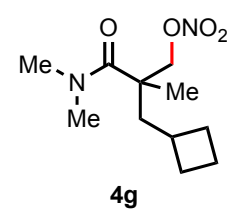

2-(cyclobutylmethyl)-3-(dimethylamino)-2-methyl-3-oxopropyl nitrate (4g)

Colorless oil. (11.2 mg, 46\%), ${ }^{1} \mathrm{H}$ NMR $\left(600 \mathrm{MHz}, \mathrm{CDCl}_{3}\right) \delta 4.7(\mathrm{~d}, J=10.3 \mathrm{~Hz}, 1 \mathrm{H})$, $4.5(\mathrm{~d}, J=10.3 \mathrm{~Hz}, 1 \mathrm{H}), 3.0(\mathrm{~s}, 6 \mathrm{H}), 2.3-2.3(\mathrm{~m}, 1 \mathrm{H}), 2.1-2.0(\mathrm{~m}, 2 \mathrm{H}), 1.9-1.5(\mathrm{~m}$, $6 \mathrm{H}), 1.4(\mathrm{~s}, 3 \mathrm{H}) .{ }^{13} \mathrm{C}$ NMR $\left(151 \mathrm{MHz}, \mathrm{CDCl}_{3}\right) \delta 173.2,77.6,46.0,42.1,38.2,32.7$, 30.2, 29.5, 21.7, 19.0., HRMS (ESI-TOF) Calcd for $\mathrm{C}_{11} \mathrm{H}_{21} \mathrm{~N}_{2} \mathrm{O}_{4}{ }^{+}[\mathrm{M}+\mathrm{H}]^{+}:$245.1501, found: 245.1507 .

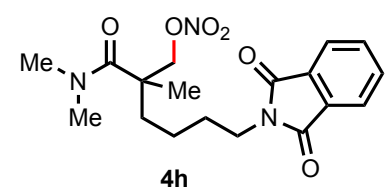

2-(dimethylcarbamoyl)-6-(1,3-dioxoisoindolin-2-yl)-2-methylhexyl nitrate (4h) Colorless oil. (23 mg, 61\%), ${ }^{1} \mathrm{H}$ NMR $\left(600 \mathrm{MHz}, \mathrm{CDCl}_{3}\right) \delta 7.9-7.8(\mathrm{~m}, 2 \mathrm{H}), 7.7-7.7$ $(\mathrm{m}, 2 \mathrm{H}), 4.7(\mathrm{~d}, J=10.5 \mathrm{~Hz}, 1 \mathrm{H}), 4.5(\mathrm{~d}, J=10.5 \mathrm{~Hz}, 1 \mathrm{H}), 3.7-3.6(\mathrm{~m}, 2 \mathrm{H}), 3.0(\mathrm{~s}$, $6 \mathrm{H}), 1.8-1.6(\mathrm{~m}, 4 \mathrm{H}), 1.4(\mathrm{~s}, 3 \mathrm{H}), 1.4-1.3(\mathrm{~m}, 1 \mathrm{H}), 1.3-1.2(\mathrm{~m}, 1 \mathrm{H}) .{ }^{13} \mathrm{C}$ NMR $(151$ $\left.\mathrm{MHz}, \mathrm{CDCl}_{3}\right) \delta 172.9,168.5,134.1,132.2,123.4,77.2,46.0,38.1,37.4,34.7,28.9$, 21.8, 21.5., HRMS (ESI-TOF) Calcd for $\mathrm{C}_{18} \mathrm{H}_{24} \mathrm{~N}_{3} \mathrm{O}_{6}{ }^{+}[\mathrm{M}+\mathrm{H}]^{+}:$378.1665, found: 378.1664 .

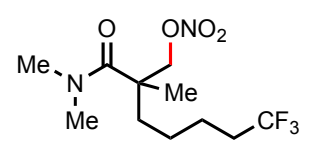

$4 \mathbf{i}$

2-(dimethylcarbamoyl)-7,7,7-trifluoro-2-methylheptyl nitrate (4i)

Yellow oil. (16.2 mg, 54\%), ${ }^{1} \mathrm{H}$ NMR $\left(600 \mathrm{MHz}, \mathrm{CDCl}_{3}\right) \delta 4.7(\mathrm{~d}, J=10.6 \mathrm{~Hz}, 1 \mathrm{H})$, $4.6(\mathrm{~d}, J=10.6 \mathrm{~Hz}, 1 \mathrm{H}), 3.0(\mathrm{~s}, 6 \mathrm{H}), 2.1-2.0(\mathrm{~m}, 2 \mathrm{H}), 1.7-1.7(\mathrm{~m}, 2 \mathrm{H}), 1.6-1.5(\mathrm{~m}$, $2 \mathrm{H}), 1.4-1.4(\mathrm{~m}, 1 \mathrm{H}), 1.39(\mathrm{~s}, 3 \mathrm{H}), 1.3-1.2(\mathrm{~m}, 1 \mathrm{H}) .{ }^{13} \mathrm{C} \mathrm{NMR}\left(151 \mathrm{MHz}, \mathrm{CDCl}_{3}\right) \delta$ 172.9, 77.0, 46.1, 38.1, 35.0, 33.7 (q, $J=28.4 \mathrm{~Hz}$ ), 24.0, 22.4 (q, $J=3.2 \mathrm{~Hz}$ ), 21.5, 20.5., ${ }^{19} \mathrm{~F}$ NMR (376 MHz, $\left.\mathrm{CDCl}_{3}\right) \delta-66.29$. HRMS (ESI-TOF) Calcd for $\mathrm{C}_{11} \mathrm{H}_{20} \mathrm{~F}_{3} \mathrm{~N}_{2} \mathrm{O}_{4}^{+}[\mathrm{M}+\mathrm{H}]^{+}: 301.1375$, found: 301.1380 . 


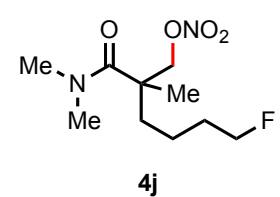

\section{2-(dimethylcarbamoyl)-6-fluoro-2-methylhexyl nitrate $(\mathbf{4 j})$}

Yellow oil. (13.2 mg, 53\%), ${ }^{1} \mathrm{H}$ NMR $\left(600 \mathrm{MHz}, \mathrm{CDCl}_{3}\right) \delta 4.7(\mathrm{~d}, J=10.6 \mathrm{~Hz}, 1 \mathrm{H})$, $4.6(\mathrm{~d}, J=10.6 \mathrm{~Hz}, 1 \mathrm{H}), 4.5-4.4(\mathrm{~m}, 1 \mathrm{H}), 4.4-4.4(\mathrm{~m}, 1 \mathrm{H}), 3.0(\mathrm{~s}, 6 \mathrm{H}), 1.8-1.6(\mathrm{~m}$, $4 \mathrm{H}), 1.5-1.4(\mathrm{~m}, 1 \mathrm{H}), 1.4(\mathrm{~s}, 3 \mathrm{H}), 1.4-1.3(\mathrm{~m}, 1 \mathrm{H}) .{ }^{13} \mathrm{C} \mathrm{NMR}\left(151 \mathrm{MHz}, \mathrm{CDCl}_{3}\right) \delta$ 173.0, 77.1, 83.7 (d, $J=165.0 \mathrm{~Hz}), 46.2,38.0,34.9,30.7$ (d, $J=19.8 \mathrm{~Hz}), 21.5 ., 20.8$ $(\mathrm{d}, J=5.0 \mathrm{~Hz}) .{ }^{19} \mathrm{~F}$ NMR $\left(376 \mathrm{MHz}, \mathrm{CDCl}_{3}\right) \delta$-218.6. HRMS (ESI-TOF) Calcd for $\mathrm{C}_{10} \mathrm{H}_{20} \mathrm{FN}_{2} \mathrm{O}_{4}{ }^{+}[\mathrm{M}+\mathrm{H}]^{+}: 251.1407$, found: 251.1410 .

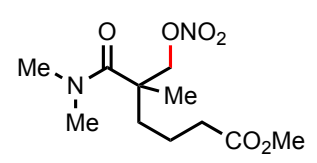

$4 \mathbf{k}$

methyl 6-(dimethylamino)-5-methyl-5-((nitrooxy)methyl)-6-oxohexanoate (4k) Colorless oil. (14.3 mg, 52\%), ${ }^{1} \mathrm{H}$ NMR $\left(400 \mathrm{MHz}, \mathrm{CDCl}_{3}\right) \delta 4.7(\mathrm{~d}, J=10.6 \mathrm{~Hz}, 1 \mathrm{H})$, $4.6(\mathrm{~d}, J=10.6 \mathrm{~Hz}, 1 \mathrm{H}), 3.7(\mathrm{~s}, 3 \mathrm{H}), 3.1(\mathrm{~s}, 6 \mathrm{H}), 2.4-2.3(\mathrm{~m}, 2 \mathrm{H}), 1.8-1.5(\mathrm{~m}, 4 \mathrm{H})$, $1.4(\mathrm{~s}, 3 \mathrm{H}) .{ }^{13} \mathrm{C} \mathrm{NMR}\left(151 \mathrm{MHz}, \mathrm{CDCl}_{3}\right) \delta 173.5,172.8,76.9,51.8,46.02,38.0,34.5$, 33.8, 21.5, 20.1. HRMS (ESI-TOF) Calcd for $\mathrm{C}_{11} \mathrm{H}_{21} \mathrm{~N}_{2} \mathrm{O}_{6}{ }^{+}[\mathrm{M}+\mathrm{H}]^{+}: 277.1400$, found: 277.1404 .

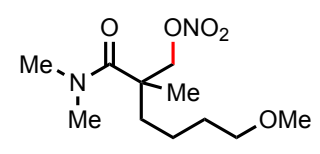

4

2-(dimethylcarbamoyl)-6-methoxy-2-methylhexyl nitrate (4I)

Colorless oil. (10.5 mg, 40\%), ${ }^{1} \mathrm{H}$ NMR $\left(600 \mathrm{MHz}, \mathrm{CDCl}_{3}\right) \delta 4.7(\mathrm{~d}, J=10.5 \mathrm{~Hz}, 1 \mathrm{H})$, $4.6(\mathrm{~d}, J=10.5 \mathrm{~Hz}, 1 \mathrm{H}), 3.4-3.4(\mathrm{~m}, 2 \mathrm{H}), 3.3(\mathrm{~s}, 3 \mathrm{H}), 3.1(\mathrm{~s}, 6 \mathrm{H}), 1.8-1.7(\mathrm{~m}, 2 \mathrm{H})$, $1.6-1.5(\mathrm{~m}, 1 \mathrm{H}), 1.3-1.2(\mathrm{~m}, 1 \mathrm{H}) .1 .4-1.3(\mathrm{~m}, 1 \mathrm{H}), 1.4(\mathrm{~s}, 3 \mathrm{H}), 1.3-1.2(\mathrm{~m}, 1 \mathrm{H})$. ${ }^{13} \mathrm{C}$ NMR $\left(151 \mathrm{MHz}, \mathrm{CDCl}_{3}\right) \delta 173.2,77.2,72.4,58.8,46.2,38.0,35.1,30.0,21.6$, 21.5., HRMS (ESI-TOF) Calcd for $\mathrm{C}_{11} \mathrm{H}_{23} \mathrm{~N}_{2} \mathrm{O}_{5}{ }^{+}[\mathrm{M}+\mathrm{H}]^{+}:$: 263.1607, found: 263.1612 .

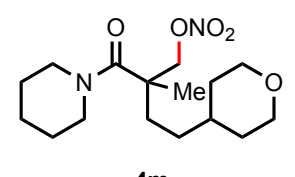

2-methyl-2-(piperidine-1-carbonyl)-4-(tetrahydro-2 $\mathrm{H}$-pyran-4-yl)butyl nitrate (4 m)

Colorless oil. (22.3 mg, 68\%), ${ }^{1} \mathrm{H}$ NMR $\left(600 \mathrm{MHz}, \mathrm{CDCl}_{3}\right) \delta 4.7(\mathrm{~d}, J=10.7 \mathrm{~Hz}, 1 \mathrm{H})$, $4.6(\mathrm{~d}, J=10.7 \mathrm{~Hz}, 1 \mathrm{H}), 4.0-3.9(\mathrm{~m}, 2 \mathrm{H}), 3.6(\mathrm{t}, J=5.5 \mathrm{~Hz}, 4 \mathrm{H}), 3.3(\mathrm{tt}, J=11.8,1.6$ $\mathrm{Hz}, 2 \mathrm{H}), 1.7-1.6(\mathrm{~m}, 4 \mathrm{H}), 1.6-1.6(\mathrm{~m}, 5 \mathrm{H}), 1.4(\mathrm{ddq}, J=14.6,6.9,3.6,3.2 \mathrm{~Hz}, 1 \mathrm{H})$, $1.4(\mathrm{~s}, 3 \mathrm{H}), 1.3-1.2(\mathrm{~m}, 4 \mathrm{H}), 1.2-1.1(\mathrm{~m}, 1 \mathrm{H}) .{ }^{13} \mathrm{C} \mathrm{NMR}\left(151 \mathrm{MHz}, \mathrm{CDCl}_{3}\right) \delta 171.6$, 
77.0, 68.1, 68.1 , 46.0, 46.0, 35.5, 33.2, 33.1, 32.3, 31.9, 26.3, 24.7, 21.8., HRMS (ESITOF) Calcd for $\mathrm{C}_{16} \mathrm{H}_{29} \mathrm{~N}_{2} \mathrm{O}_{5}{ }^{+}[\mathrm{M}+\mathrm{H}]^{+}: 329.2076$, found: 329.2083 .<smiles>CN1CCCC(CO[Na])C1=O</smiles>

$4 n$

\section{(1-methyl-2-oxopiperidin-3-yl)methyl nitrate (4n)}

Yellow oil. (10 mg, 53\%), ${ }^{1} \mathrm{H}$ NMR $\left(400 \mathrm{MHz}, \mathrm{CDCl}_{3}\right) \delta 4.8(\mathrm{dd}, J=10.4,3.9 \mathrm{~Hz}, 1 \mathrm{H})$, $4.8(\mathrm{dd}, J=10.4,6.8 \mathrm{~Hz}, 1 \mathrm{H}), 3.4-3.2(\mathrm{~m}, 2 \mathrm{H}), 3.0(\mathrm{~s}, 3 \mathrm{H}), 2.7-2.8(\mathrm{~m}, 1 \mathrm{H}), 2.1-$ $1.9(\mathrm{~m}, 2 \mathrm{H}), 1.9-1.6(\mathrm{~m}, 2 \mathrm{H}) .{ }^{13} \mathrm{C}$ NMR $\left(151 \mathrm{MHz}, \mathrm{CDCl}_{3}\right) \delta 168.0,73.6,50.0,40.3$, 35.1, 24.8, 22.0., HRMS (ESI-TOF) Calcd for $\mathrm{C}_{7} \mathrm{H}_{13} \mathrm{~N}_{2} \mathrm{O}_{4}{ }^{+}[\mathrm{M}+\mathrm{H}]^{+}:$188.0875, found: 188.0879 .<smiles>CN1CCC(C)(C(F)(F)F)CC(C)(CO[N+](=O)[O-])C1=O</smiles>

\section{(5,5-difluoro-1,3-dimethyl-2-oxoazepan-3-yl)methyl nitrate (4o)}

White solid. (18.4 mg, 73\%), m.p. $103{ }^{\circ} \mathrm{C} .{ }^{1} \mathrm{H}$ NMR $\left(600 \mathrm{MHz}, \mathrm{CDCl}_{3}\right) \delta 4.8(\mathrm{~d}, J=$ $10.5 \mathrm{~Hz}, 1 \mathrm{H}), 4.4$ (d, $J=10.5 \mathrm{~Hz}, 1 \mathrm{H}), 3.7$ (ddt, $J=16.0,10.8,1.1 \mathrm{~Hz}, 1 \mathrm{H}), 3.4$ (ddt, $J$ $=16.0,6.7,2.2 \mathrm{~Hz}, 1 \mathrm{H}), 3.1(\mathrm{~s}, 3 \mathrm{H}), 2.4-2.1(\mathrm{~m}, 4 \mathrm{H}), 1.4(\mathrm{~d}, J=2.8 \mathrm{~Hz}, 3 \mathrm{H}) .{ }^{13} \mathrm{C}$ NMR $\left(151 \mathrm{MHz}, \mathrm{CDCl}_{3}\right) \delta 173.0,123.5(\mathrm{dd}, J=241.4,239.4 \mathrm{~Hz}), 78.6,44.4(\mathrm{dd}, J=$ 9.5, 3.0 Hz), 43.6 (d, $J=10.1 \mathrm{~Hz}), 40.0$ (t, $J=25.9 \mathrm{~Hz}), 38.9,36.6(\mathrm{t}, J=25.4 \mathrm{~Hz})$, 20.2., ${ }^{19} \mathrm{~F}$ NMR $\left(376 \mathrm{MHz}, \mathrm{CDCl}_{3}\right) \delta-83.90(\mathrm{~d}, J=252.6 \mathrm{~Hz}),-93.88(\mathrm{~d}, J=252.6$ $\mathrm{Hz}$ )., HRMS (ESI-TOF) Calcd for $\mathrm{C}_{9} \mathrm{H}_{15} \mathrm{~F}_{2} \mathrm{~N}_{2} \mathrm{O}_{4}{ }^{+}[\mathrm{M}+\mathrm{H}]^{+}:$253.1000, found: 253.1002 .

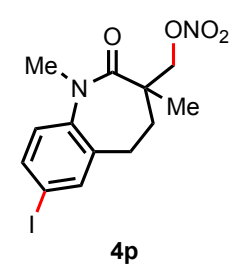

(7-iodo-1,3-dimethyl-2-oxo-2,3,4,5-tetrahydro-1 $H$-benzo[b]azepin-3-yl)methyl nit rate $(4 p)$

Colorless oil. (19.9 mg, 51\%), ${ }^{1} \mathrm{H}$ NMR $\left(600 \mathrm{MHz}, \mathrm{CDCl}_{3}\right) \delta 7.6(\mathrm{dd}, J=8.4,2.0 \mathrm{~Hz}$, $1 \mathrm{H}), 7.5(\mathrm{~d}, J=2.0 \mathrm{~Hz}, 1 \mathrm{H}), 6.9(\mathrm{~d}, J=8.4 \mathrm{~Hz}, 1 \mathrm{H}), 4.5(\mathrm{~d}, J=10.2 \mathrm{~Hz}, 1 \mathrm{H}), 4.3(\mathrm{~d}, J$ $=10.2 \mathrm{~Hz}, 1 \mathrm{H}), 3.4(\mathrm{~s}, 3 \mathrm{H}), 2.8(\mathrm{ddd}, J=13.7,11.8,6.2 \mathrm{~Hz}, 1 \mathrm{H}), 2.6(\mathrm{ddd}, J=13.7$, 5.7, $4.6 \mathrm{~Hz}, 1 \mathrm{H}), 2.2(\mathrm{ddd}, J=13.7,6.2,4.6 \mathrm{~Hz}, 1 \mathrm{H}), 1.9(\mathrm{ddd}, J=13.7,11.8,5.7 \mathrm{~Hz}$, $1 \mathrm{H}), 0.9(\mathrm{~s}, 3 \mathrm{H}) .{ }^{13} \mathrm{C} \mathrm{NMR}\left(151 \mathrm{MHz}, \mathrm{CDCl}_{3}\right) \delta 172.6,142.6,137.1,136.9,136.7,123.7$, 89.8, 77.6, 45.0, 40.3, 37.1, 29.1, 22.1., HRMS (ESI-TOF) Calcd for $\mathrm{C}_{13} \mathrm{H}_{16} \mathrm{IN}_{2} \mathrm{O}_{4}{ }^{+}$ $[\mathrm{M}+\mathrm{H}]^{+}:$391.0155, found: 391.0156 . 


\section{The Synthesis and Characterization of Palladacycles}

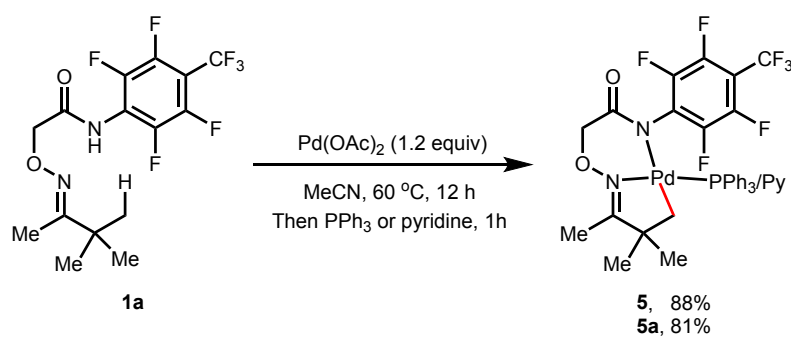

General Procedures for the Palladacycle Synthesis: Substrate 1a $(0.1 \mathrm{mmol}, 38.8$ $\mathrm{mg})$ and $\mathrm{Pd}(\mathrm{OAc})_{2}(0.12 \mathrm{mmol}, 27.0 \mathrm{mg})$ were weighed into a reaction vial $(10 \mathrm{~mL})$ with a magnetic stir bar under air. $\mathrm{MeCN}(1.0 \mathrm{~mL})$ was added, and the vial was sealed with a cap. The reaction mixture was stirred at $60^{\circ} \mathrm{C}$ in oil bath for 12 hours. Upon completion, the reaction mixture was cooled to room temperature and $\mathrm{PPh}_{3}(0.12 \mathrm{mmol}$, $31.4 \mathrm{mg}$ ) was added. The reaction was stirred for anther $1 \mathrm{~h}$ at $60^{\circ} \mathrm{C}$. Upon completion, the reaction mixture was cooled to room temperature and diluted with EtOAc. Then the reaction mixture was filtered through a plug of celite. The solvent was removed under vacuum and the resulting mixture was purified by preparative thin-layer chromatography with EtOAc : Hexane $=1: 1$ as the eluent.

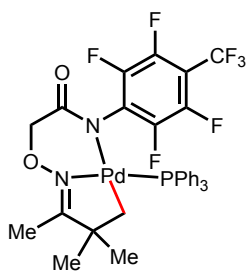

\section{Palladacycle (5)}

Substrate 1a was palladated following the general procedures. After purification by preparative thin-layer chromatography (EtOAc : Hexane $=1: 1$ as eluent), 5 was obtained as a yellow solid (66.4 mg, 88\%), m.p. $126{ }^{\circ} \mathrm{C},{ }^{1} \mathrm{H}$ NMR $\left(500 \mathrm{MHz}, \mathrm{CDCl}_{3}\right)$ $\delta 7.43(\mathrm{t}, J=7.1 \mathrm{~Hz}, 3 \mathrm{H}), 7.34(\mathrm{dd}, J=13.8,6.3 \mathrm{~Hz}, 12 \mathrm{H}), 4.81(\mathrm{~s}, 2 \mathrm{H}), 2.01(\mathrm{~s}, 3 \mathrm{H})$, $1.64(\mathrm{~d}, J=4.3 \mathrm{~Hz}, 2 \mathrm{H}), 1.21(\mathrm{~s}, 6 \mathrm{H}) .{ }^{13} \mathrm{C} \mathrm{NMR}\left(126 \mathrm{MHz}, \mathrm{CDCl}_{3}\right) \delta 176.5,169.8$, 133.6, 133.5, 130.8, 130.1, 129.7, 128.7, 128.6, 79.0, 50.4, 46.0, 29.5, 12.3. HRMS (ESI-TOF) Calcd for $\mathrm{C}_{33} \mathrm{H}_{29} \mathrm{~F}_{7} \mathrm{~N}_{2} \mathrm{O}_{2} \mathrm{PPd}^{+}[\mathrm{M}+\mathrm{H}]^{+}$: 755.0889, found: 755.0895. The structure of 5 was also confirmed by X-ray crystallography (CCDC 2076323).

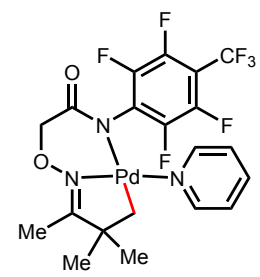

\section{Palladacycle (5a)}

Substrate 1a was palladated following the general procedures, but pyridine $(0.12 \mathrm{mmol}$, $10 \mathrm{ul}$ ) was used instead of $\mathrm{PPh}_{3}$. After purification by preparative thin-layer 
chromatography (EtOAc : Hexane $=1: 1$ as eluent), 5a was obtained as an off white solid (46.3 mg, 81\%), m.p. $80{ }^{\circ} \mathrm{C},{ }^{1} \mathrm{H}$ NMR $\left(500 \mathrm{MHz}, \mathrm{CDCl}_{3}\right) \delta 8.34(\mathrm{~d}, J=5.1 \mathrm{~Hz}$, 2H), $7.65(\mathrm{t}, J=7.7 \mathrm{~Hz}, 1 \mathrm{H}), 7.18-7.13(\mathrm{~m}, 2 \mathrm{H}), 4.59$ (s, 2H), 1.99 (s, 3H), 1.96 (s, $2 \mathrm{H}), 1.25(\mathrm{~s}, 6 \mathrm{H}) .{ }^{13} \mathrm{C} \mathrm{NMR}\left(126 \mathrm{MHz}, \mathrm{CD}_{3} \mathrm{OD}\right) \delta 184.3,170.3,151.8,138.2,125.2$, 76.1, 49.6, 37.8, 27.4, 11.8. HRMS (ESI-TOF) Calcd for $\mathrm{C}_{16} \mathrm{H}_{17} \mathrm{~F}_{7} \mathrm{~N}_{4} \mathrm{O}_{2} \mathrm{Pd}^{+}\left[\mathrm{M}+\mathrm{NH}_{4}\right]^{+}$: 536.0278, found: 536.0245. (The observed mass was in accordance with the complex where pyridine was replaced by $\mathrm{MeCN}$ in 5a.)
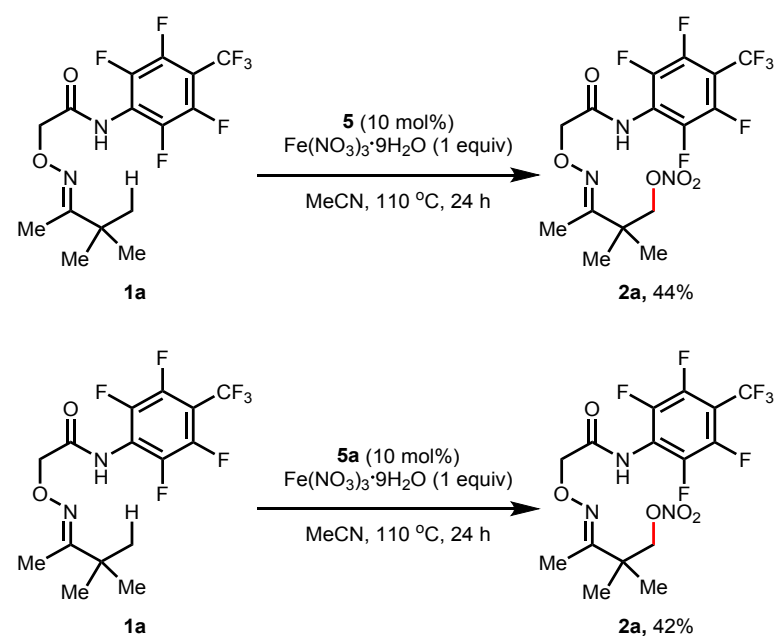

General Procedures: Substrate 1a was also nitrooxylated following the general nitrooxylation procedure, but $10 \mathrm{~mol} \%$ of 5 was used as the catalyst. After purification by preparative thin-layer chromatography (Hexane: EtOAc $=6: 1$ as eluent), 2a was obtained ( $9.9 \mathrm{mg}, 44 \%)$. The experiment with palladacycle 5a was similar as above.

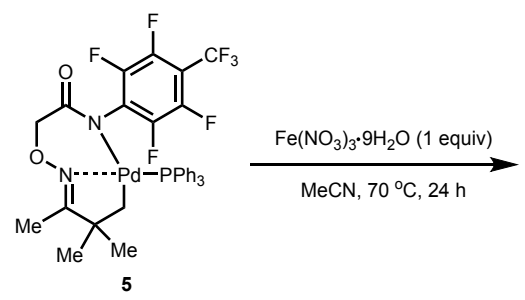

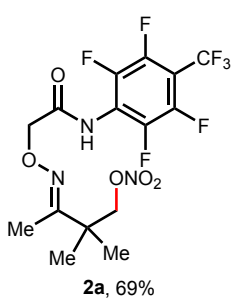
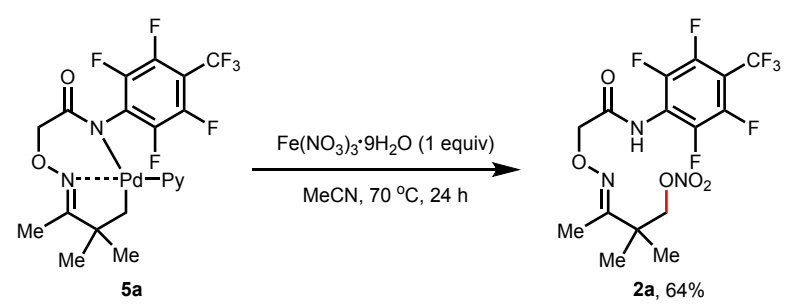

General Procedures: Palladacycle $5(0.05 \mathrm{mmol}, 37.8 \mathrm{mg})$, and $\mathrm{Fe}\left(\mathrm{NO}_{3}\right)_{3} \cdot 9 \mathrm{H}_{2} \mathrm{O}(0.05$ mmol, $20.2 \mathrm{mg})$ were weighed into a reaction vial $(10 \mathrm{~mL})$ with a magnetic stir bar under air. MeCN ( $2 \mathrm{~mL}$ ) was added, and the vial was sealed with a cap. The reaction mixture was stirred at $110^{\circ} \mathrm{C}$ in oil bath for 24 hours. Upon completion, 2a was obtained (15.5 mg, 69\%). The experiment with palladacycle 5a was similar as above. 


\section{The Removal of Axiliary and Conversion of Nitrate}
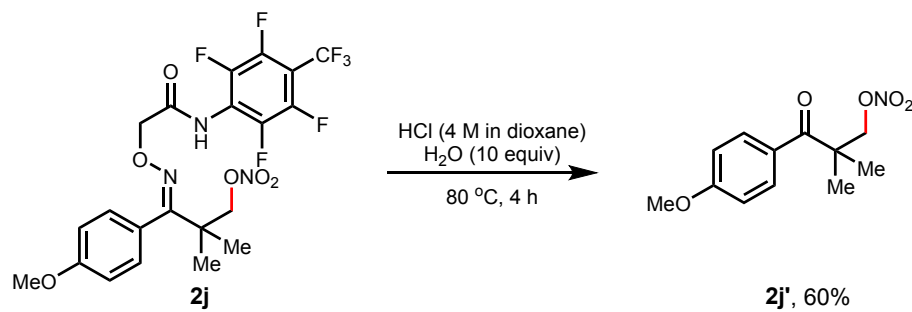

$2 \mathbf{j}^{\prime}, 60 \%$

General Procedures : $\mathbf{2 j}$ ( $0.05 \mathrm{mmol}, 27.1 \mathrm{mg})$ was weighed into a reaction vial (10 $\mathrm{mL}$ ) with a magnetic stir bar under air. $9.0 \mu \mathrm{L}$ of $\mathrm{H}_{2} \mathrm{O}$ (10 equiv) and $0.5 \mathrm{~mL}$ of $\mathrm{HCl}(4$ $\mathrm{M}$ in dioxane) were added, and the vial was sealed with a cap. The reaction mixture was stirred at $80^{\circ} \mathrm{C}$ in oil bath for 4 hours. Upon completion, the reaction mixture was cooled to room temperature and the solvent was removed under vacuum. The resulting mixture was purified by preparative thin-layer chromatography by using Hexane /EtOAc (9:1) as the eluent to give $7.6 \mathrm{mg}$ of $\mathbf{2 j}$ ' $(60 \%$ yield $)$<smiles>COc1ccc(C(=O)C(C)(C)C(=O)O[Na])cc1</smiles>

\section{3-(4-methoxyphenyl)-2,2-dimethyl-3-oxopropyl nitrate (2j')}

Colorless oil. ${ }^{1} \mathrm{H}$ NMR $\left(500 \mathrm{MHz}, \mathrm{CDCl}_{3}\right) \delta 7.82(\mathrm{~d}, J=8.9 \mathrm{~Hz}, 2 \mathrm{H}), 6.92(\mathrm{~d}, J=8.9$ $\mathrm{Hz}, 2 \mathrm{H}), 4.66$ (s, 2H), 3.87 (s, 3H), 1.49 (s, 6H). ${ }^{13} \mathrm{C} \mathrm{NMR}\left(126 \mathrm{MHz}, \mathrm{CDCl}_{3}\right) \delta 202.2$, 162.7, 130.9, 129.1, 113.6, 78.7, 55.5, 46.9, 23.5. HRMS (EI) Calcd for $\mathrm{C}_{12} \mathrm{H}_{15} \mathrm{NO}_{5} \mathrm{Na}^{+}$ $[\mathrm{M}+\mathrm{Na}]^{+}: 276.0848$, found: 276.0845 .
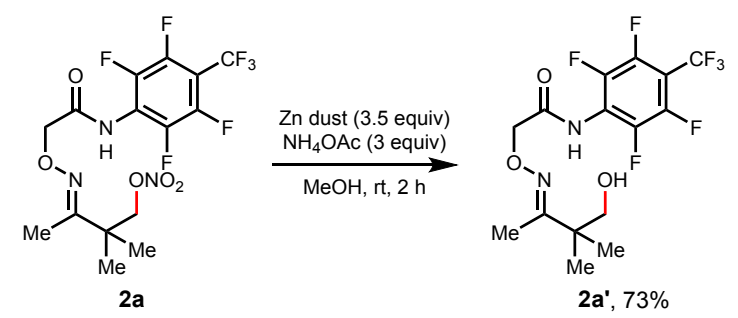

General Procedures: ${ }^{10}$ Substrate $2 a(0.1 \mathrm{mmol}, 44.9 \mathrm{mg})$, Zn dust $(0.35 \mathrm{mmol}, 22.9$ $\mathrm{mg})$ and $\mathrm{NH}_{4} \mathrm{OAc}(0.3 \mathrm{mmol}, 23.1 \mathrm{mg})$ were weighed into a reaction vial $(10 \mathrm{~mL})$ with a magnetic stir bar under air. $\mathrm{MeOH}(2 \mathrm{~mL})$ was added, and the vial was sealed with a cap. The reaction mixture was stirred at room temperature. Upon completion, the reaction mixture was cooled to room temperature and diluted with EtOAc. Then the reaction mixture was filtered through a plug of silicon gel. The solvent was removed under vacuum. The resulting mixture was purified by preparative thin-layer chromatography by using Hexane/EtOAc (6:1) as the eluent to give $29.5 \mathrm{mg}$ of $\mathbf{2 a}$ ' $(73 \%$ yield). 


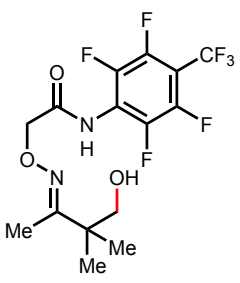

(E)-2-(((4-hydroxy-3,3-dimethylbutan-2-ylidene)amino)oxy)-N-(2,3,5,6-tetrafluor o-4-(trifluoromethyl)phenyl)acetamide (2a')

Colorless oil. ${ }^{1} \mathrm{H}$ NMR $\left(500 \mathrm{MHz}, \mathrm{CDCl}_{3}\right) \delta 7.98(\mathrm{~s}, 1 \mathrm{H}), 4.73(\mathrm{~s}, 2 \mathrm{H}), 3.58(\mathrm{~s}, 2 \mathrm{H})$, $1.96(\mathrm{~s}, 3 \mathrm{H}), 1.14(\mathrm{~s}, 6 \mathrm{H}) .{ }^{13} \mathrm{C} \mathrm{NMR}\left(126 \mathrm{MHz}, \mathrm{CDCl}_{3}\right) \delta 168.4,166.8,72.6,69.6,42.8$, 22.5, 11.3. HRMS (ESI-TOF) Calcd for $\mathrm{C}_{15} \mathrm{H}_{16} \mathrm{~F}_{7} \mathrm{~N}_{2} \mathrm{O}_{2+}[\mathrm{M}+\mathrm{H}]^{+}: 405.1049$, found: 405.1045 . 


\section{X-Ray Crystallographic Data of $2 b$ and 5}

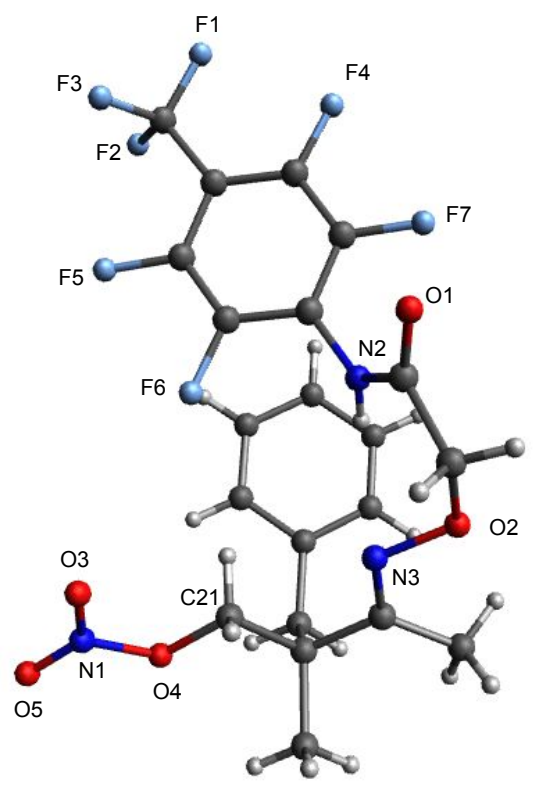

Crystal data and structure refinement for $2 b$. (CCDC 2076322)

Report date

Identification code

Empirical formula

Formula weight

Temperature

Wavelength

Crystal system

Space group

Unit cell dimensions

Volume

Z

Density (calculated)

Absorption coefficient

$\mathrm{F}(000)$

Crystal size

Theta range for data collection

Index ranges

Reflections collected

Independent reflections
2020-06-14

20200614LI_NLG_JIANGCHAO_0m_a

C21 H18 F7 N3 O5

525.38

193(2) K

$1.34139 \AA$

Triclinic

P-1

$$
\begin{array}{ll}
\mathrm{a}=9.0926(2) \AA & \alpha=79.2960(10)^{\circ} . \\
\mathrm{b}=10.8538(3) \AA & \beta=87.9550(10)^{\circ} . \\
\mathrm{c}=11.6733(3) \AA & \gamma=81.1810(10)^{\circ} .
\end{array}
$$

1118.57(5) $\AA^{3}$

2

$1.560 \mathrm{Mg} / \mathrm{m}^{3}$

$0.846 \mathrm{~mm}^{-1}$

536

$0.120 \times 0.110 \times 0.080 \mathrm{~mm}^{3}$

3.352 to $53.958^{\circ}$.

$-10<=\mathrm{h}<=10,-13<=\mathrm{k}<=13,-14<=\mathrm{l}<=14$

14280

$4069[\mathrm{R}($ int $)=0.0348]$ 
Completeness to theta $=53.594^{\circ}$

Refinement method

Data / restraints / parameters

Goodness-of-fit on $\mathrm{F}^{2}$

Final $\mathrm{R}$ indices [I $>2 \operatorname{sigma}(\mathrm{I})]$

$\mathrm{R}$ indices (all data)

Extinction coefficient

Largest diff. peak and hole
$99.7 \%$

Full-matrix least-squares on $\mathrm{F}^{2}$

4069 / 0 / 327

1.083

$\mathrm{R} 1=0.0534, \mathrm{wR} 2=0.1558$

$\mathrm{R} 1=0.0584, \mathrm{wR} 2=0.1606$

$\mathrm{n} / \mathrm{a}$

0.571 and -0.319 e. $\AA^{-3}$

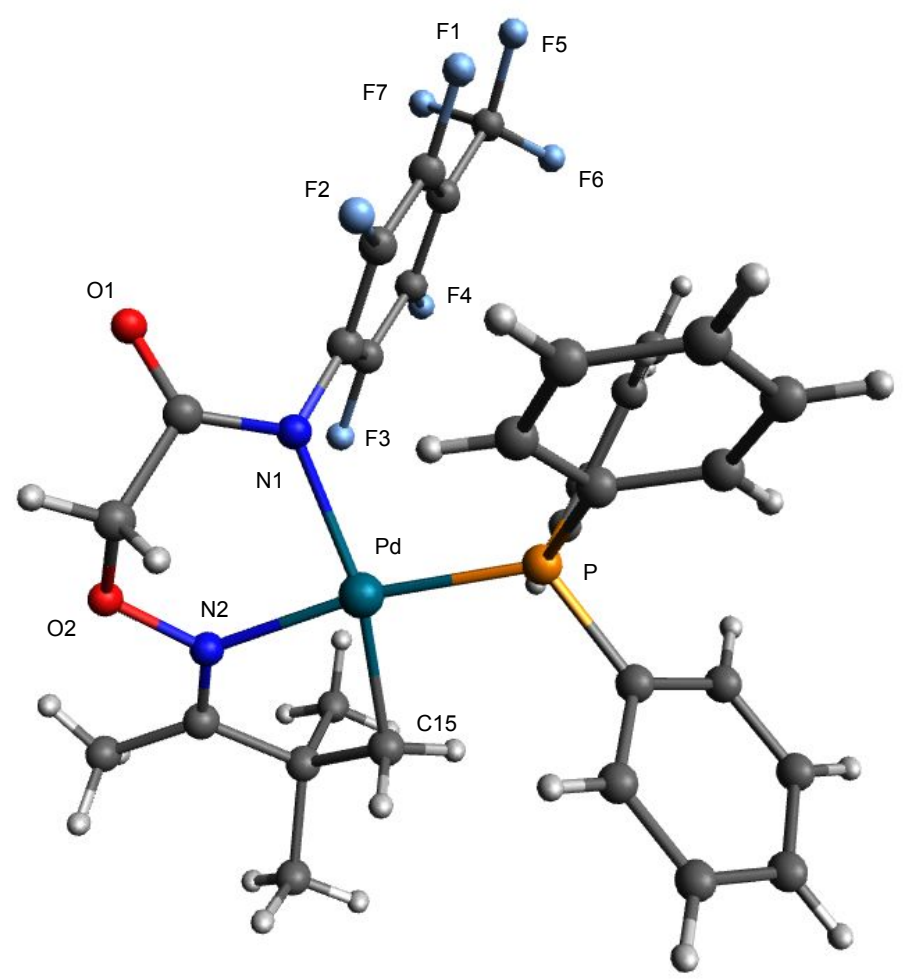

Crystal data and structure refinement for 5. (CCDC 2076323)

Report date

Identification code

Empirical formula

Formula weight

Temperature

Wavelength

Crystal system

Space group

Unit cell dimensions

Volume
2019-09-05

20190905LI_NLG_JC3a_1_0m_a

C33 H28 F7 N2 O2 P Pd

754.94

296(2) K

$0.71073 \AA$

Triclinic

P -1

$\mathrm{a}=10.2240(3) \AA$ $\alpha=102.4920(10)^{\circ}$.

$\mathrm{b}=15.6567(5) \AA$ $\beta=90.6450(10)^{\circ}$.

$\mathrm{c}=20.8337(7) \AA$ $\gamma=99.7460(10)^{\circ}$. 
Z

Density (calculated)

Absorption coefficient

$\mathrm{F}(000)$

Crystal size

Theta range for data collection

Index ranges

Reflections collected

Independent reflections

Completeness to theta $=25.010^{\circ}$

Refinement method

Data / restraints / parameters

Goodness-of-fit on $\mathrm{F}^{2}$

Final $\mathrm{R}$ indices [I $>2 \operatorname{sigma}(\mathrm{I})]$

$\mathrm{R}$ indices (all data)

Extinction coefficient

Largest diff. peak and hole
4

$1.565 \mathrm{Mg} / \mathrm{m}^{3}$

$0.703 \mathrm{~mm}^{-1}$

1520

$0.160 \times 0.110 \times 0.090 \mathrm{~mm}^{3}$

2.005 to $25.010^{\circ}$.

$-12<=\mathrm{h}<=11,-18<=\mathrm{k}<=18,-17<=\mathrm{l}<=24$

23917

$11187[\mathrm{R}(\mathrm{int})=0.0460]$

$99.0 \%$

Full-matrix least-squares on $\mathrm{F}^{2}$

11187 / 139 / 920

1.041

$\mathrm{R} 1=0.0371, \mathrm{wR} 2=0.0820$

$\mathrm{R} 1=0.0496, \mathrm{wR} 2=0.0912$

$\mathrm{n} / \mathrm{a}$

0.338 and -0.478 e. $\AA^{-3}$ 


\section{References}

(1) Peter, M.; Gleiter, R.; Rominger, F.; Oeser, T. Eur. J. Org. Chem. 2004, 3212.

(2) Yang, Q. L.; Li, Y. Q.; Ma, C.; Fang, P.; Zhang, X. J.; Mei, T. S. J. Am. Chem. Soc. 2017, 139, 3293.

(3) Mandal, R.; Emayavaramban, B.; Sundararaju, B. Org. Lett. 2018, 20, 2835-2838.

(4) Ushijima, S.; Dohi, S.; Moriyama, K.; Togo, H. Tetrahedron. 2012, 68, 1436.

(5) Li, X.; Li, L.; Tang, Y.; Zhong, L.; Cun, L.; Zhu, J.; Liao, J.; Deng, J. J. Org. Chem. 2010 , 75, 2981.

(6) Chambers, R. D.; Martin, P. A.; Sandford, G.; Williams, D. L. H. J. Fluorine Chem. 2008, 129, 998.

(7) Park, H.; Yang, L.; Yu, J.-Q. Angew. Chem. Int Ed., 2019, 58, 11424.

(8) Park, H.; Chekshin, N.; Shen, P.-X.; Yu, J.-Q. ACS Catal. 2018, 8.

(9) Park, H.; Yu, J.-Q. J. Am. Chem. Soc. 2020, 142, 16552.

(10) Thurow, S.; Fernandes, A. A. G.; Quevedo-Acosta,Y.; De Oliveira, M. F.; De Oliveira Marcelo G.; Jurberg. Org. Lett. 2019, 21, 6909. 


\section{${ }^{1} \mathrm{H}$ and ${ }^{13} \mathrm{C}$ NMR Spectra}

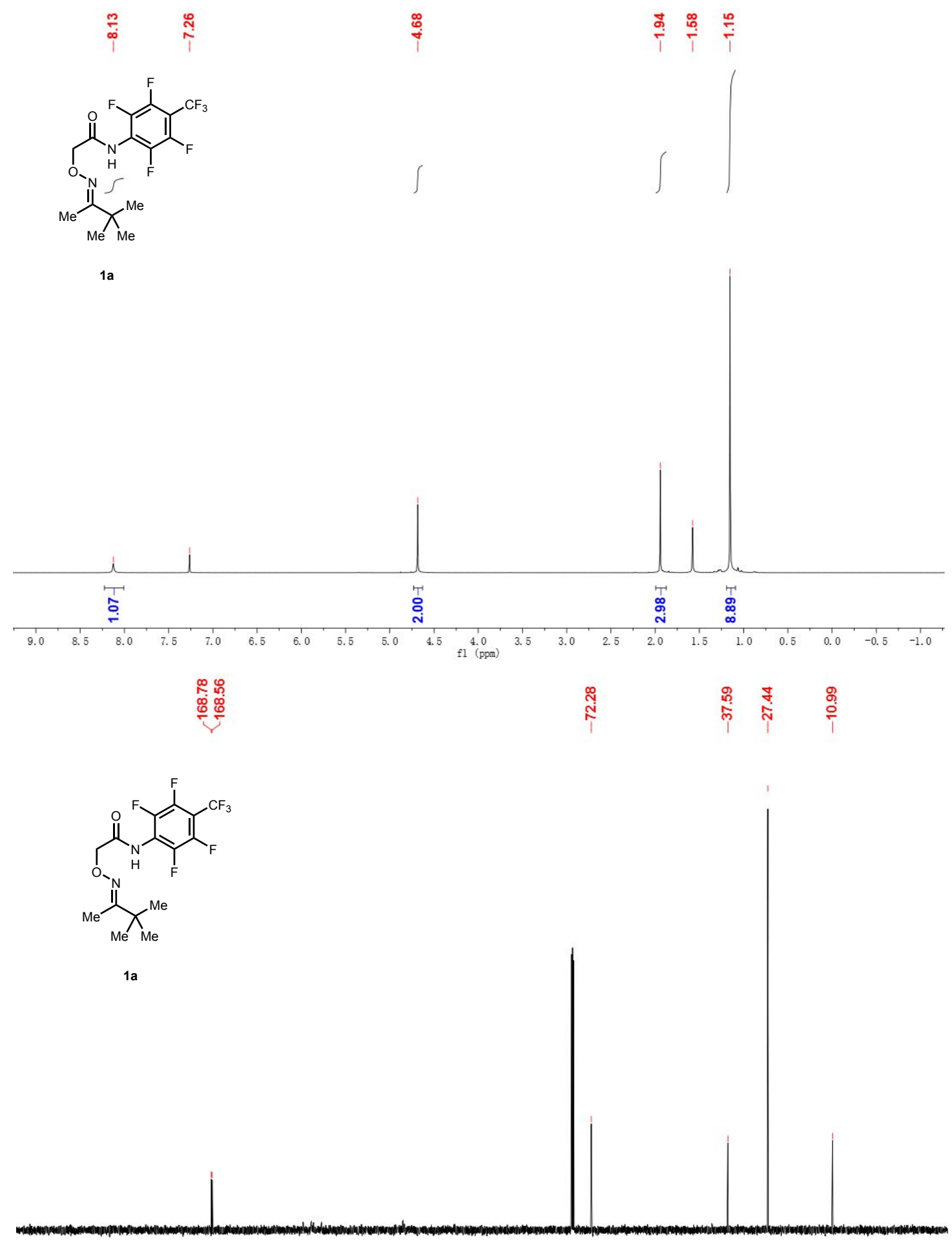

$\begin{array}{llllllllllll}210 & 200 & 190 & 180 & 170 & 160 & 150 & 140 & 130 & 120 & 110 & 100 \\ \mathrm{f} 1(\mathrm{ppm}) & 90\end{array}$ 


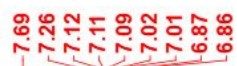

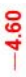

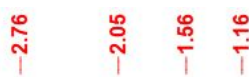<smiles>C=Cc1c(F)c(F)c(F)c(NC(=O)COC(C)C(C)(C)C(C)(C)C)c1F</smiles>

ᄃ $]$

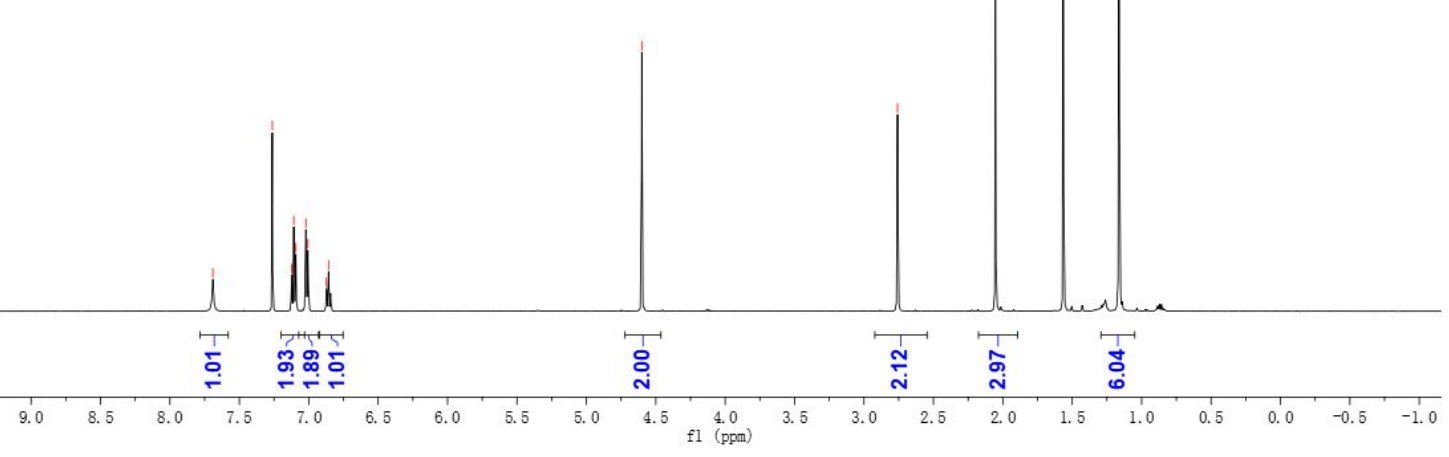

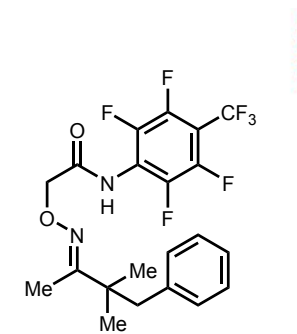

1b

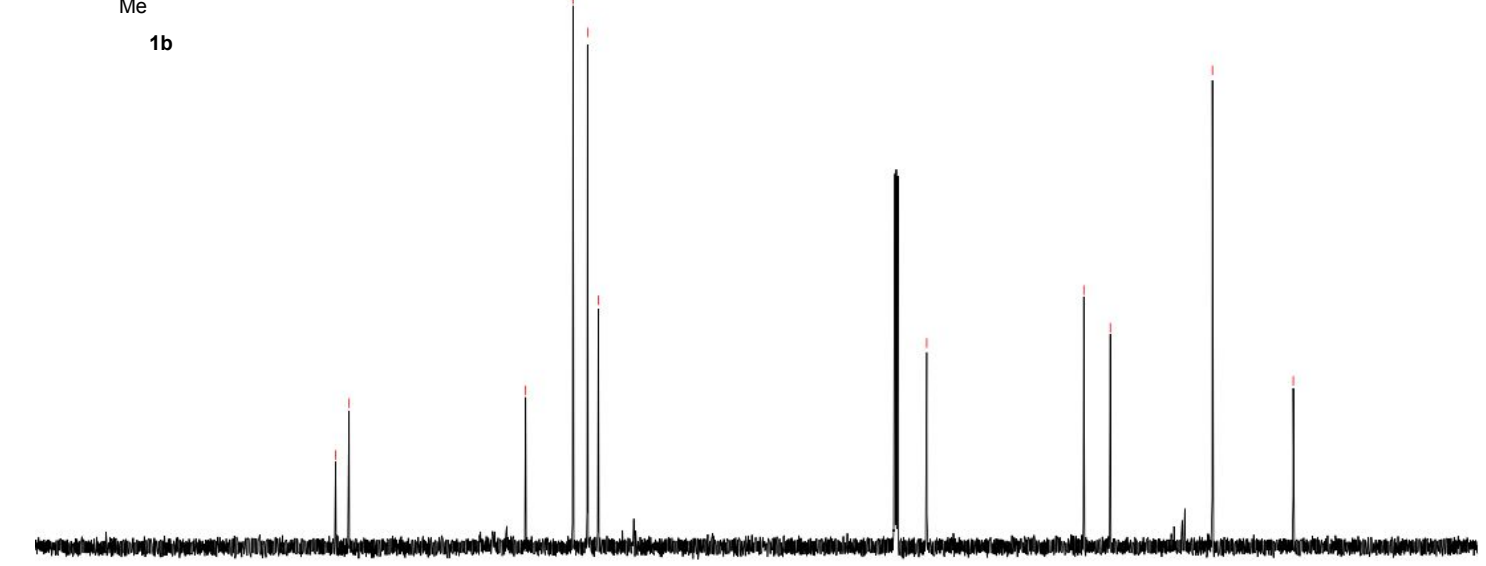

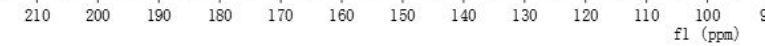



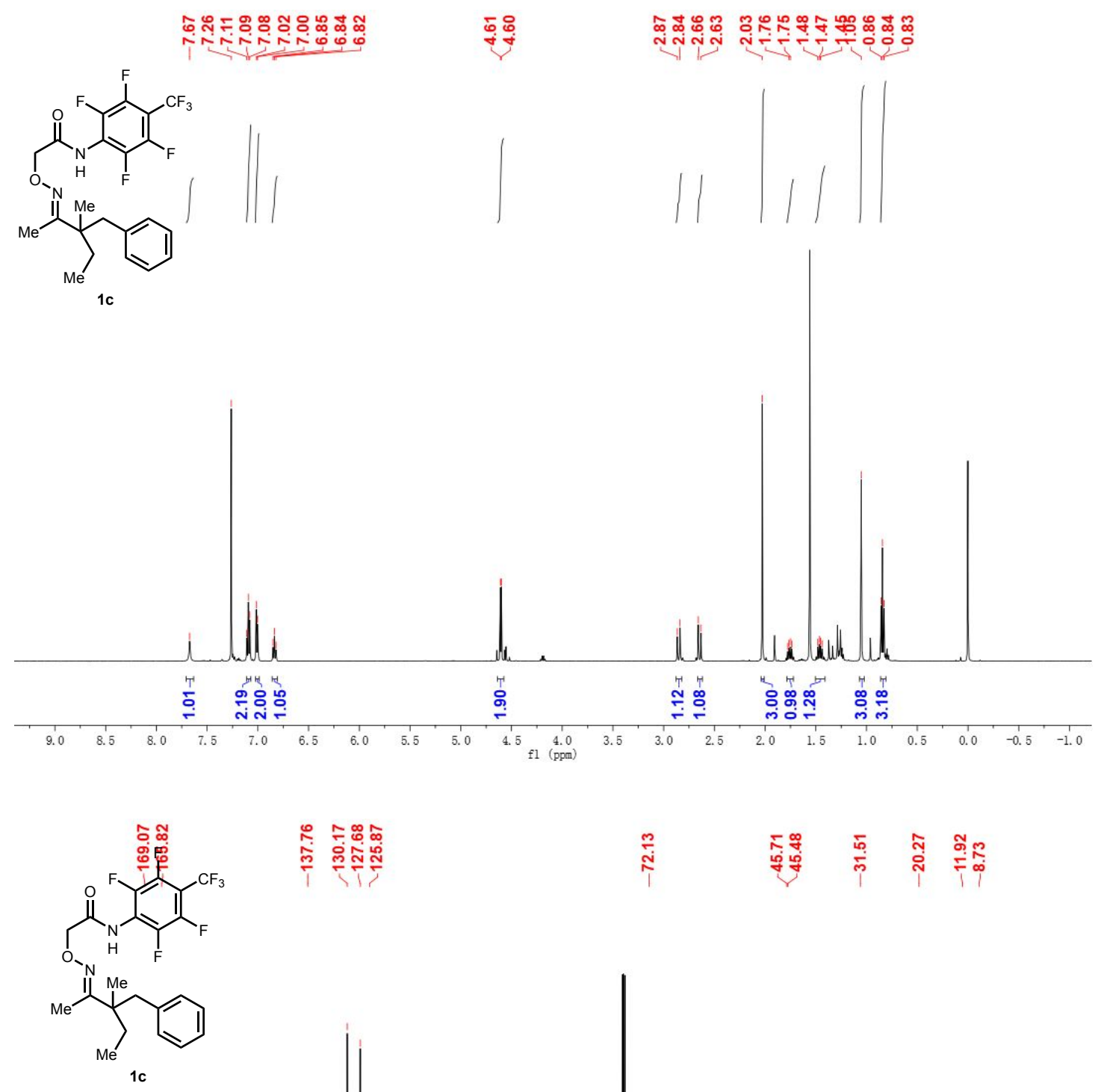

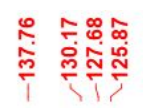

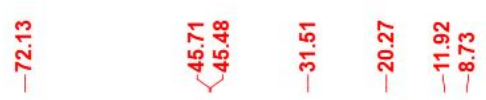

$\begin{array}{lllllllllll}190 & 180 & 170 & 160 & 150 & 140 & 130 & 120 & 110 & 100 & 90 \\ \text { f1 (ppm) }\end{array}$ 


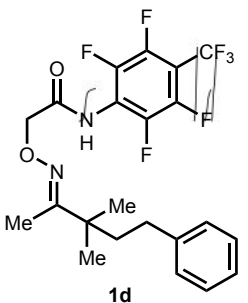
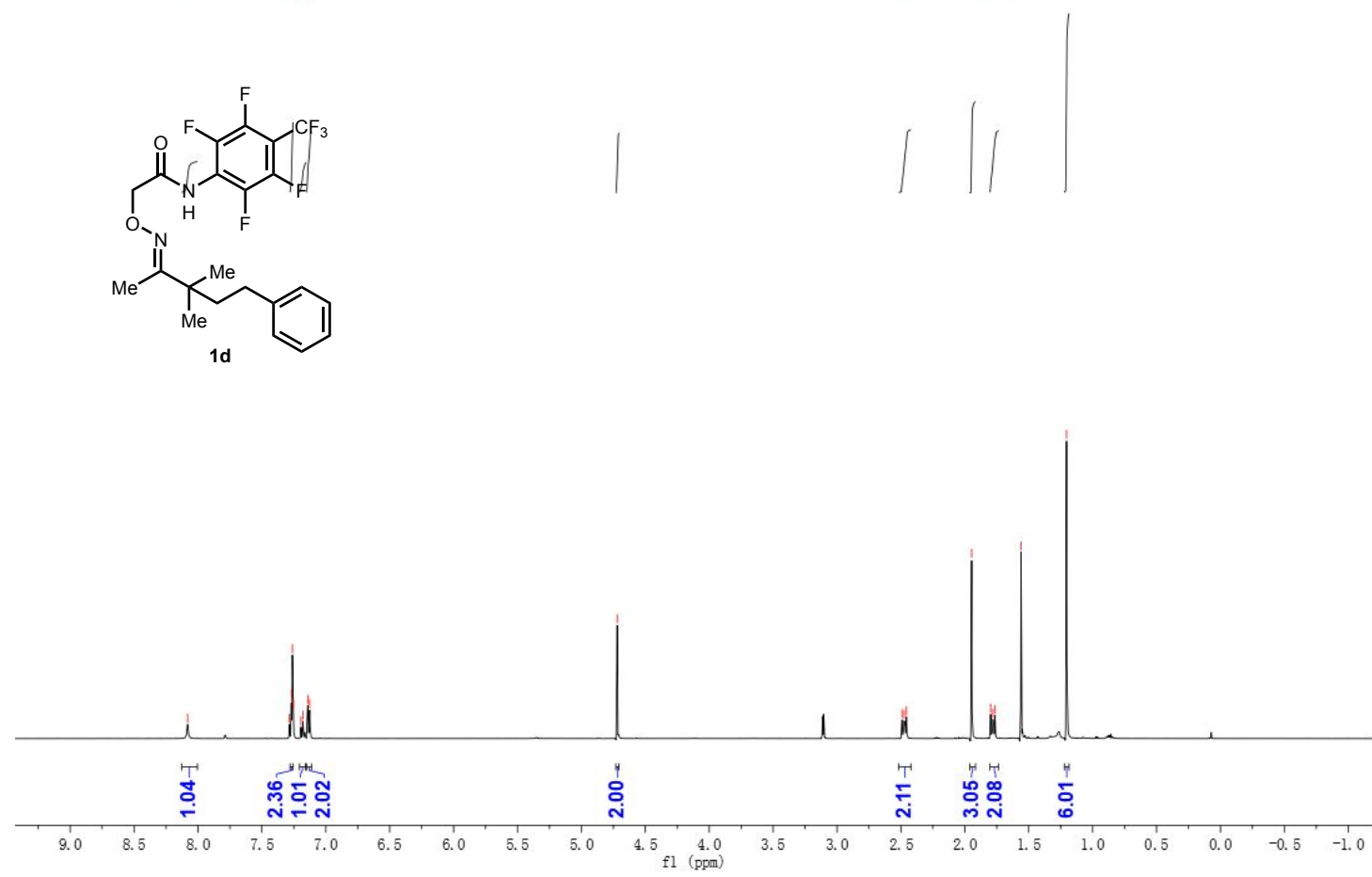<smiles>C/C(=C/OCC(=O)N/N=C(/C)C(C)(C)Cc1ccccc1)Nc1c(F)c(F)c(F)c(P)c1F</smiles>

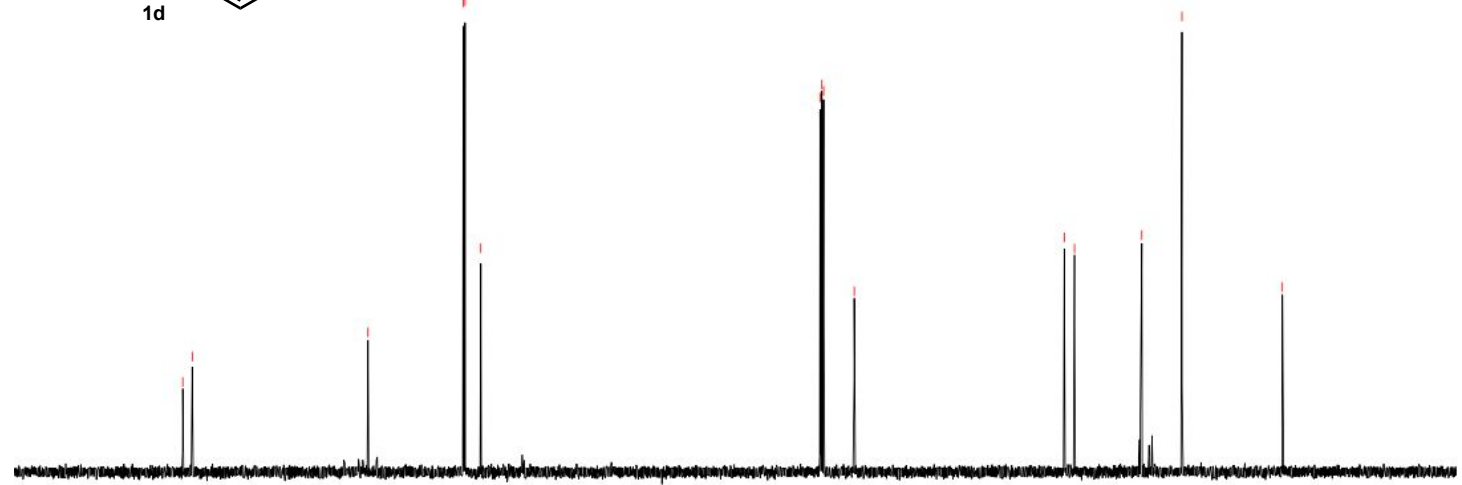

190

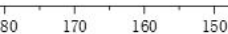

11
140

$100 \stackrel{90}{90}$

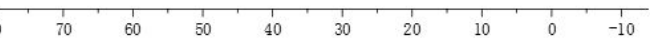




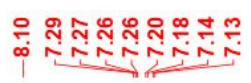

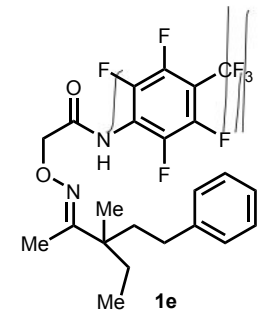

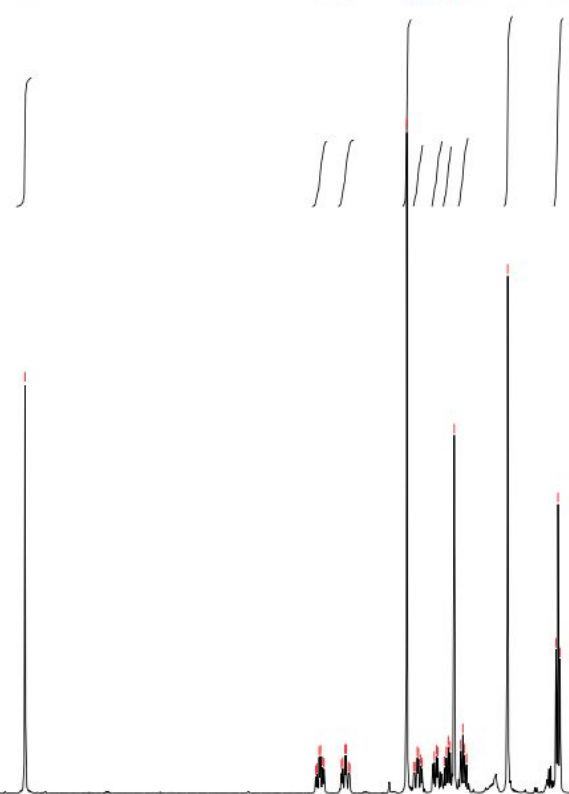
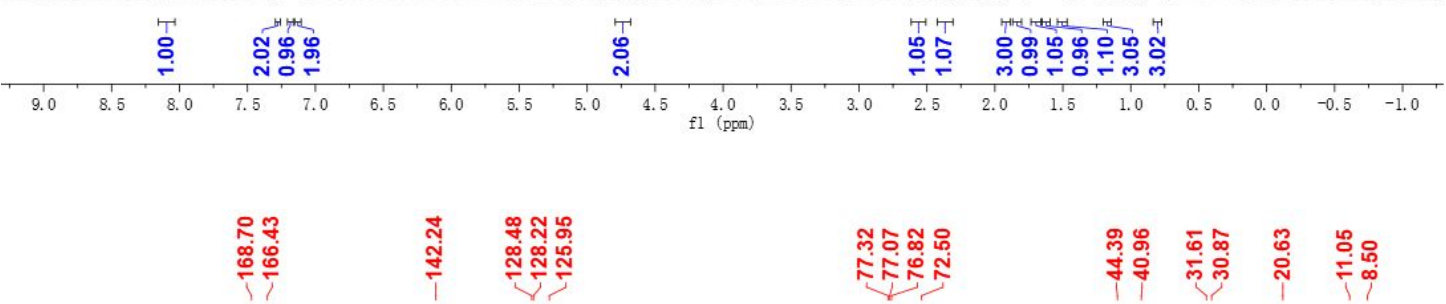

\section{ำำำ}

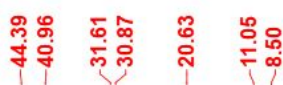<smiles>CCCC(CC)(CC)C(C)=NOCC(=O)Nc1c(F)c(F)c(F)c(F)c1F</smiles>
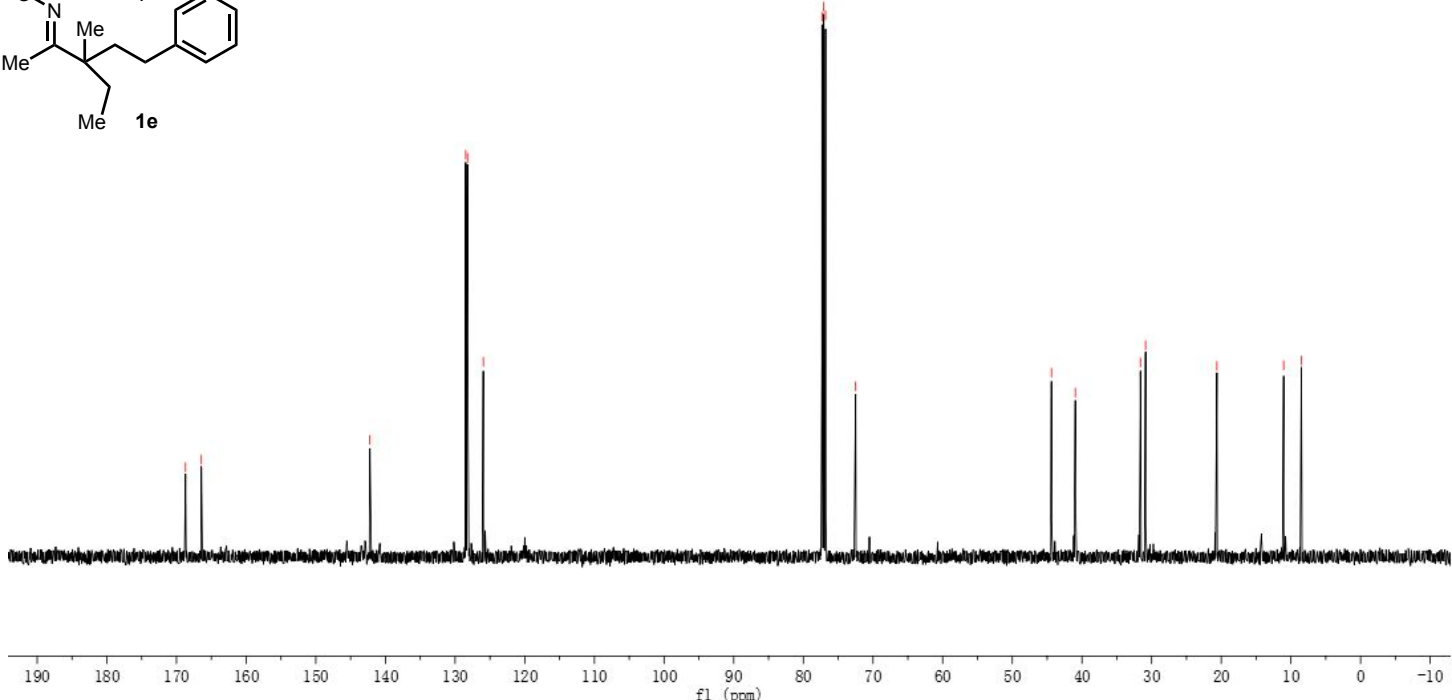

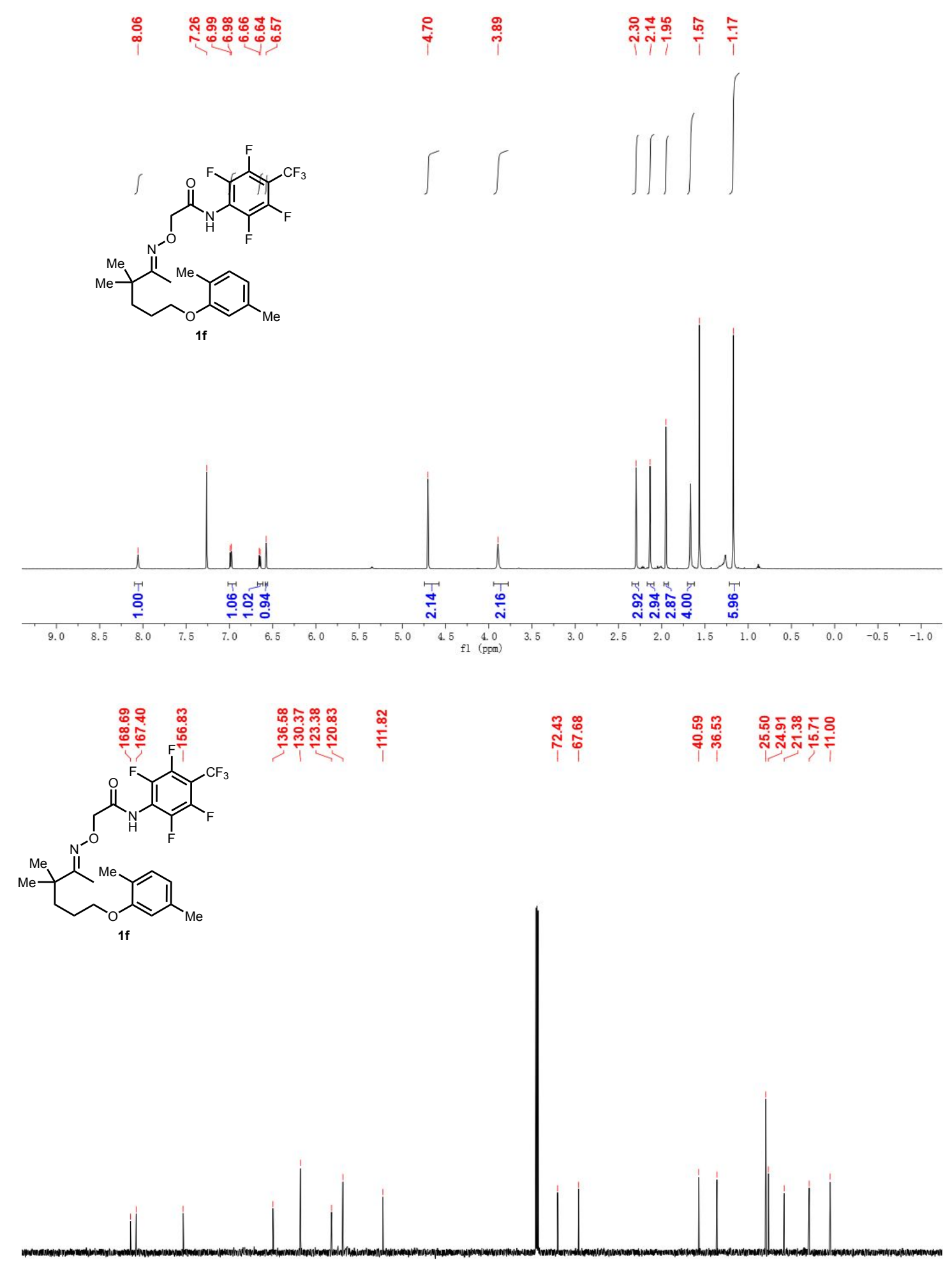

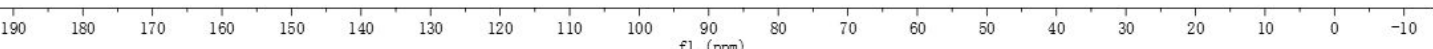



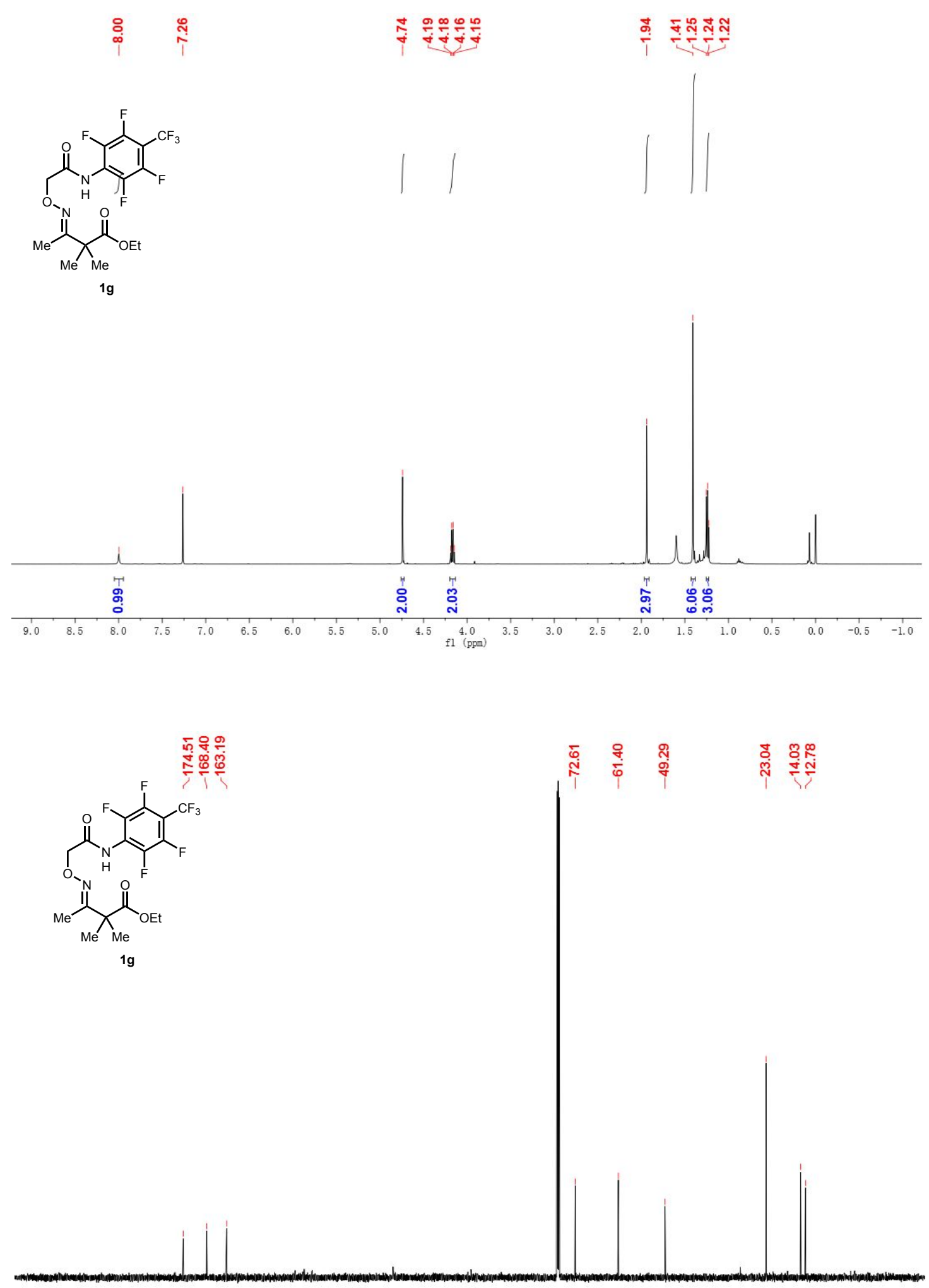

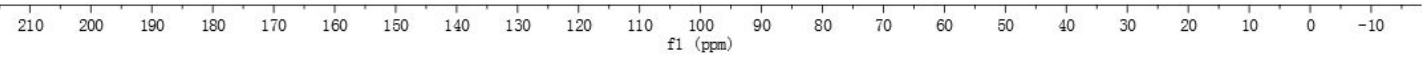



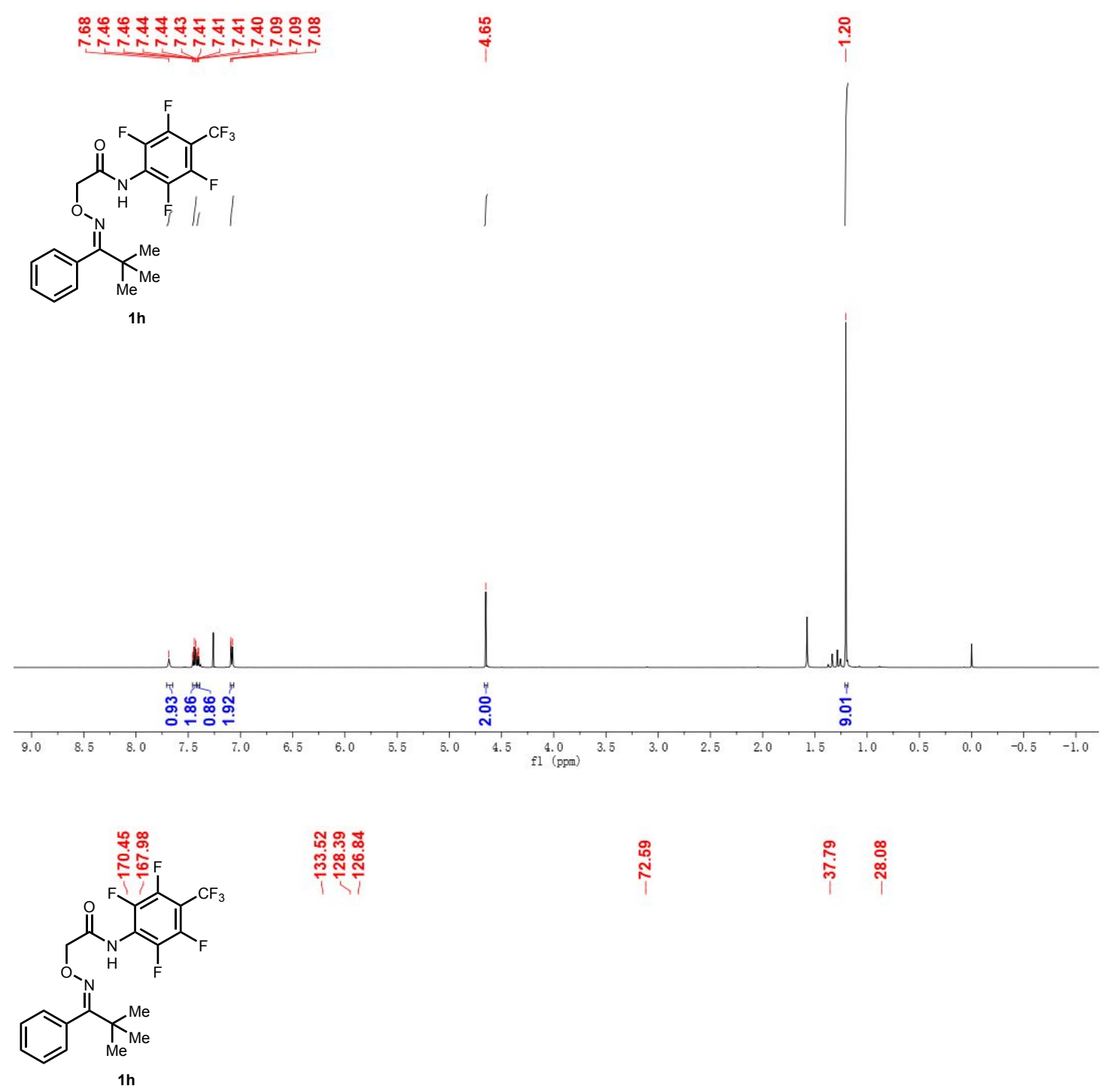

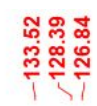
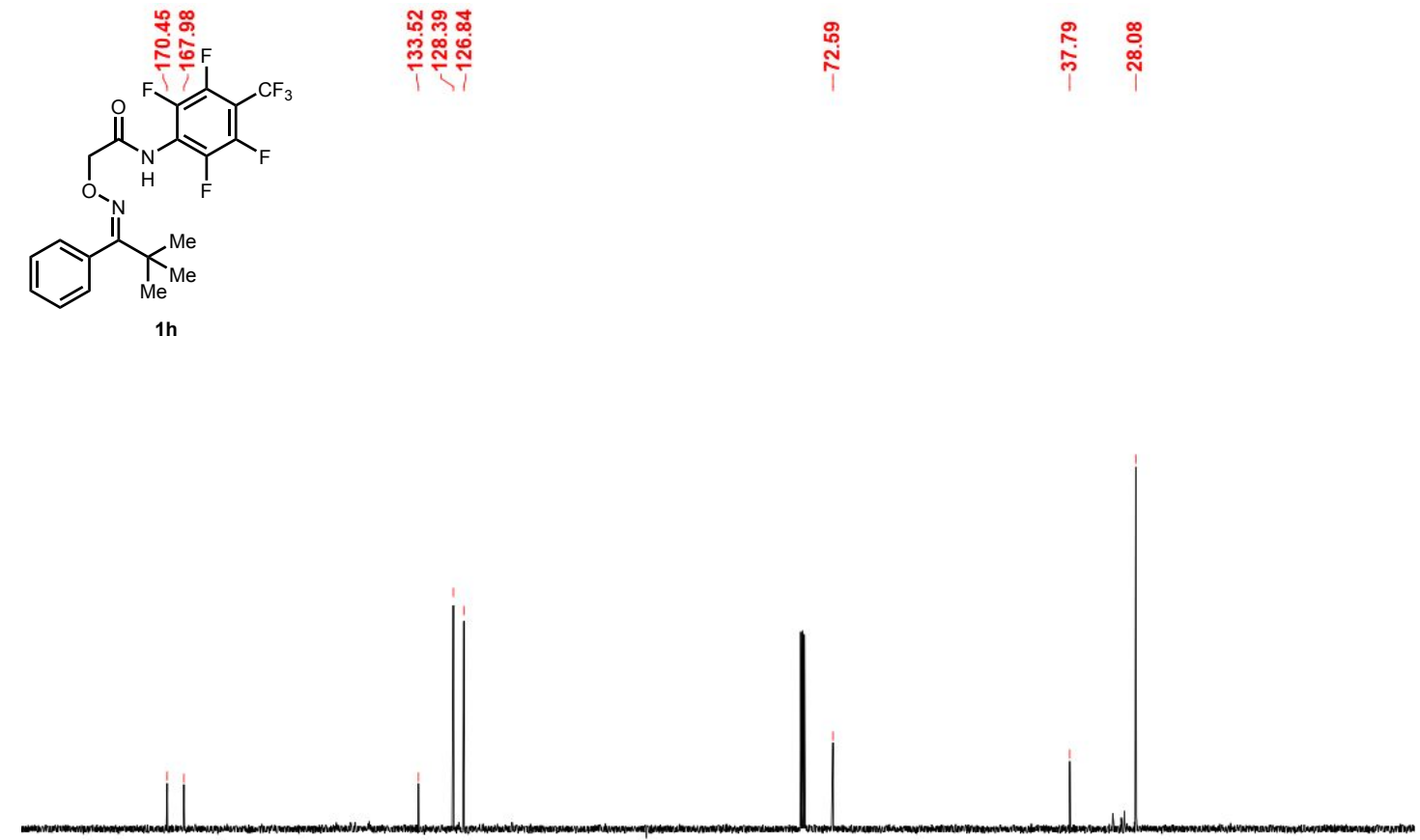

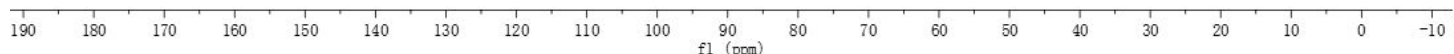



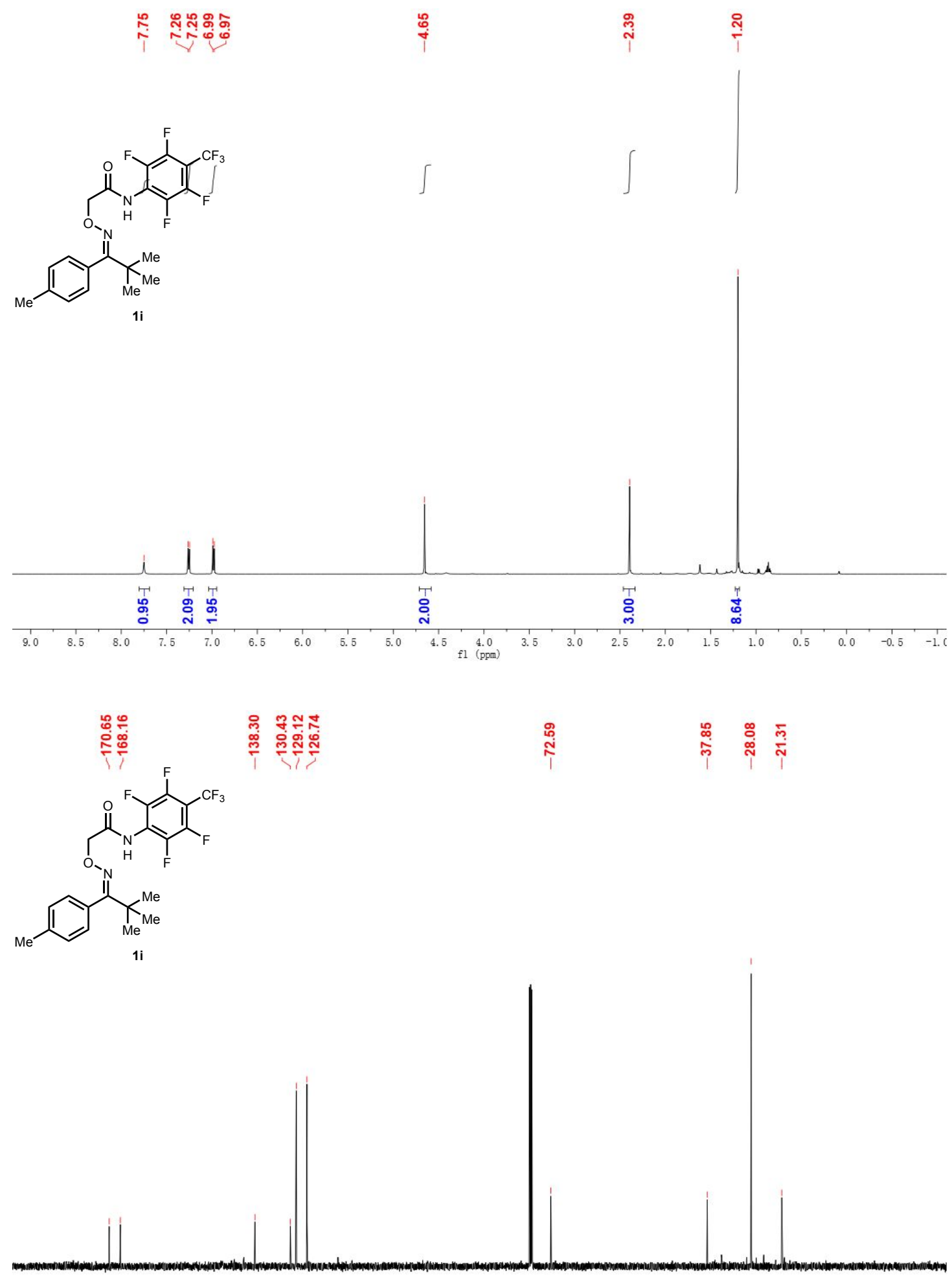

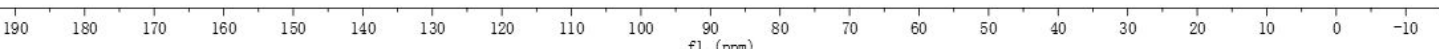



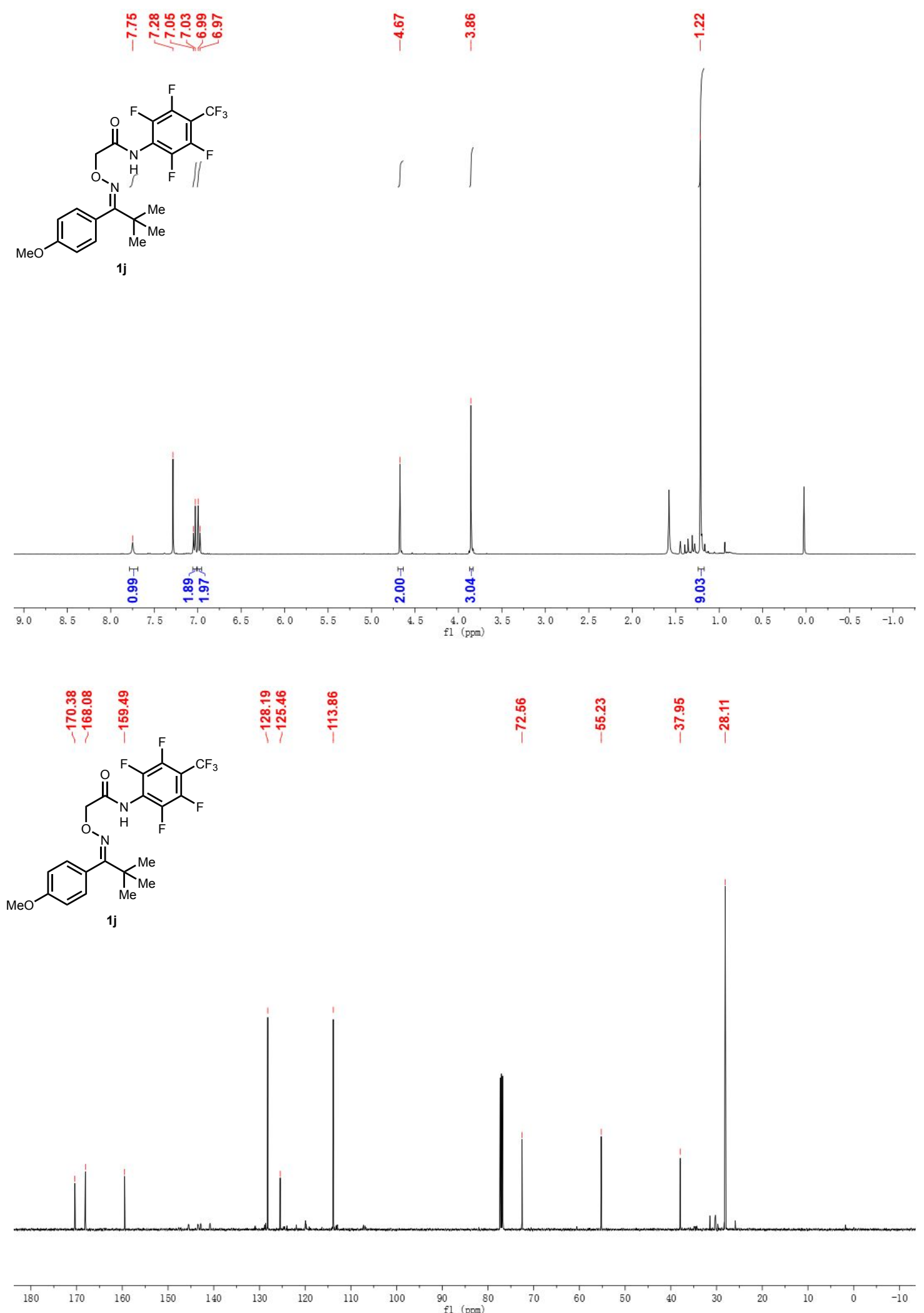

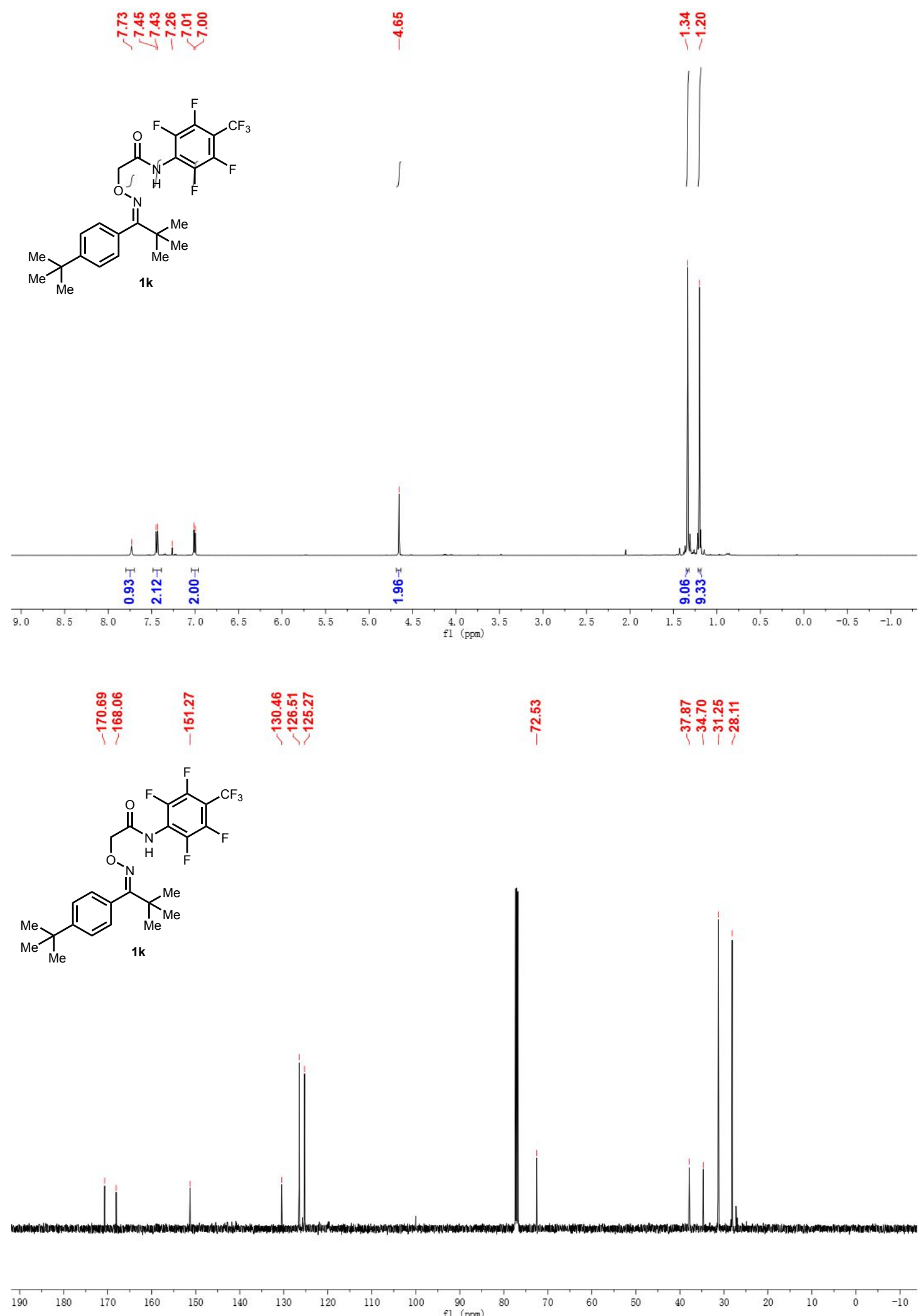

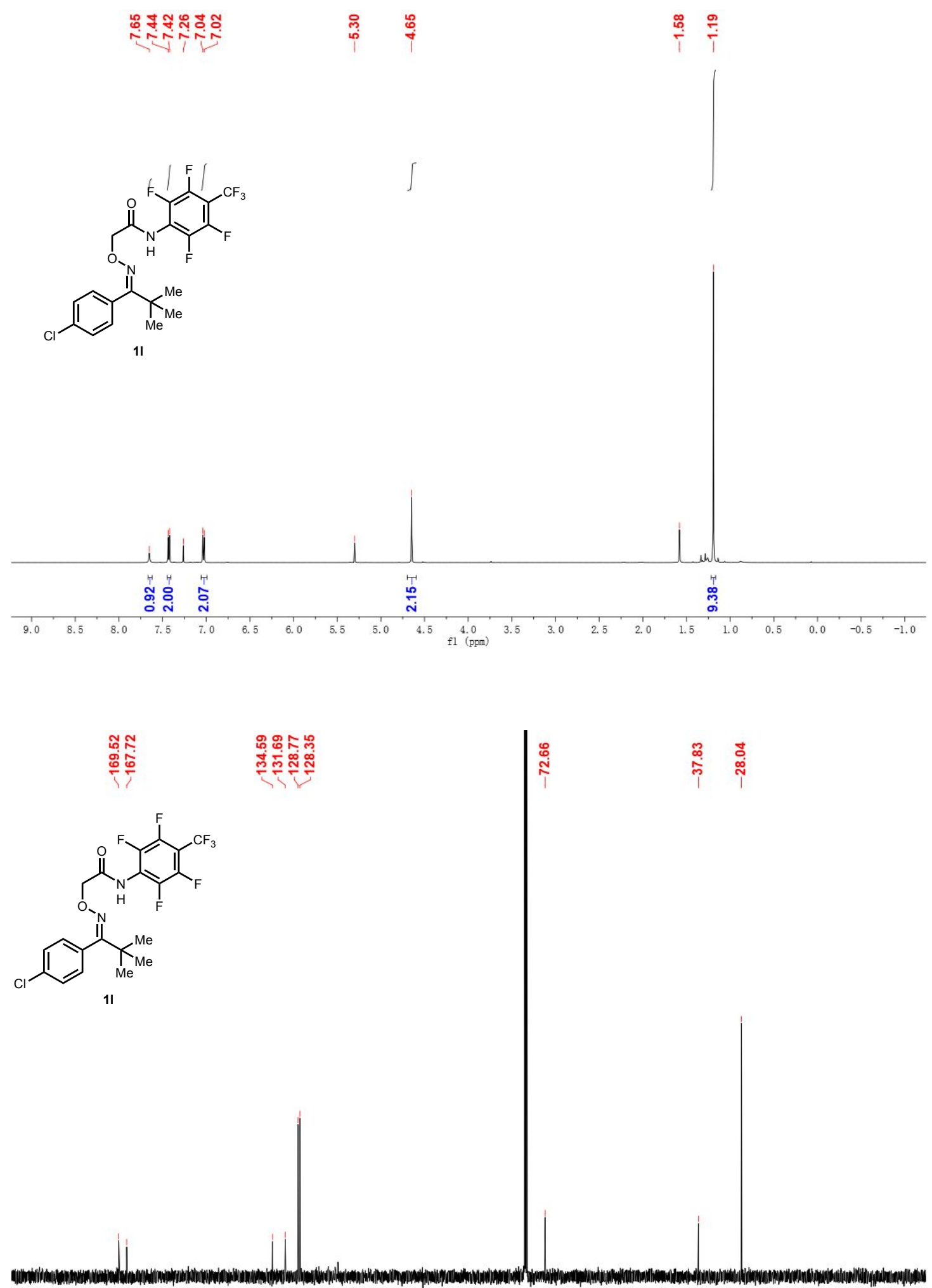

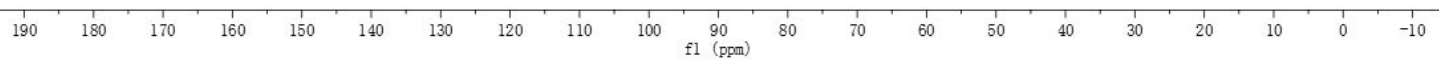



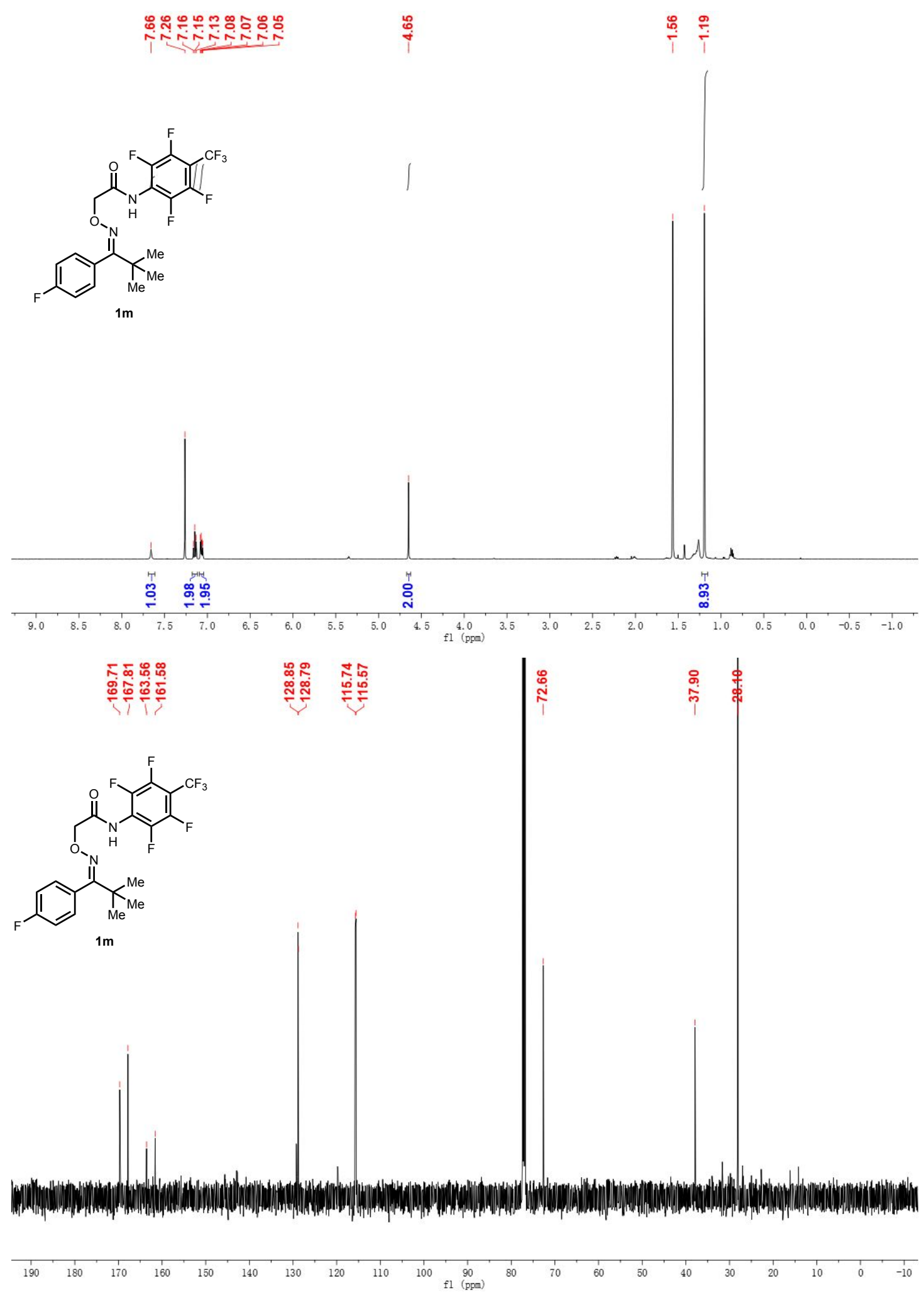

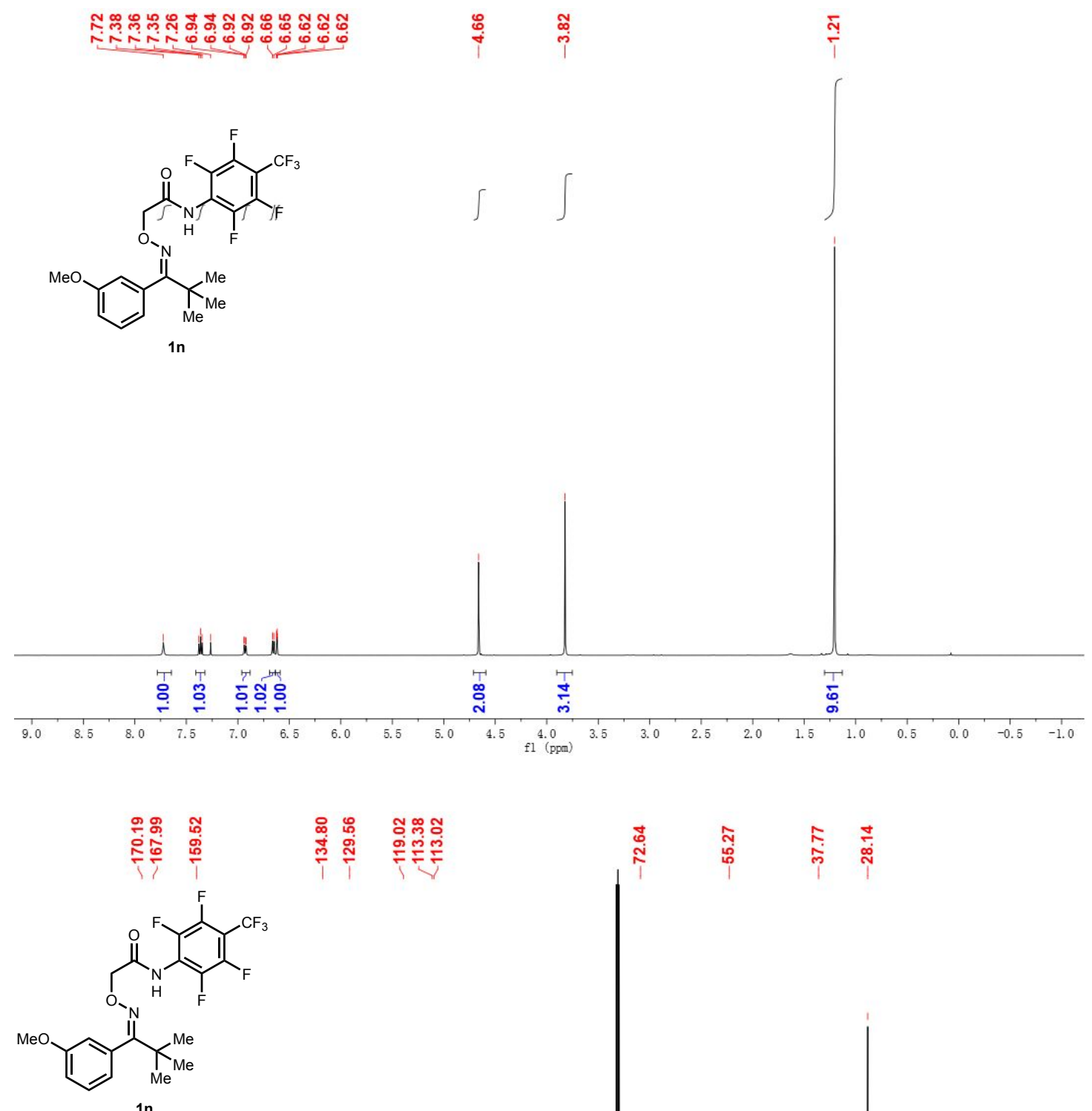

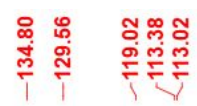

î

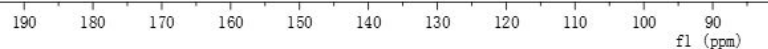




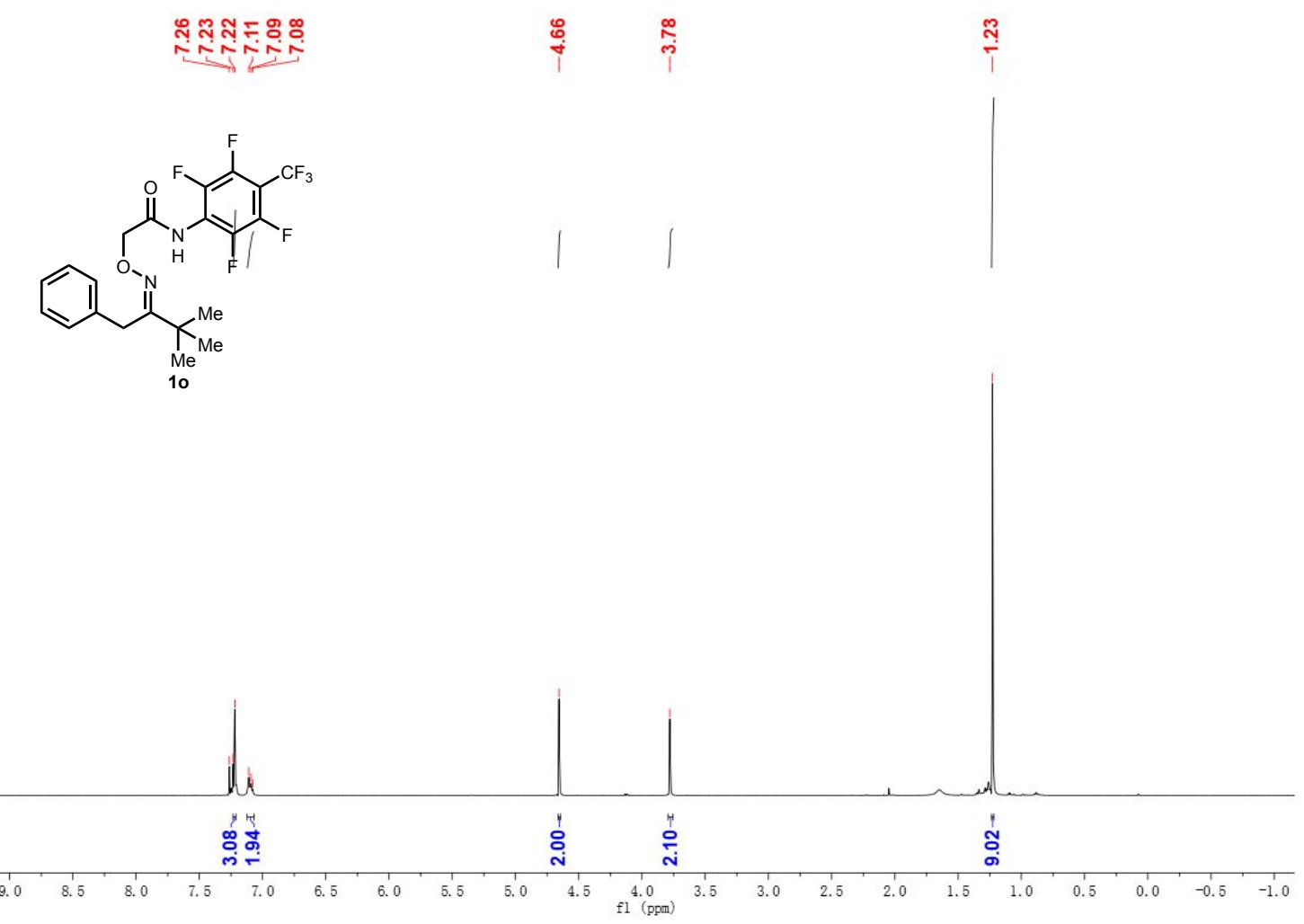

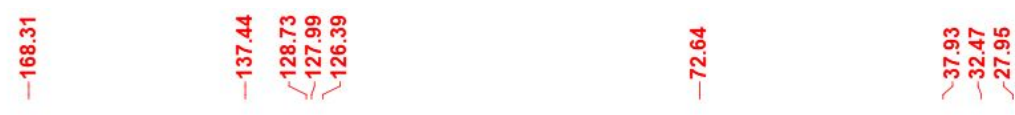
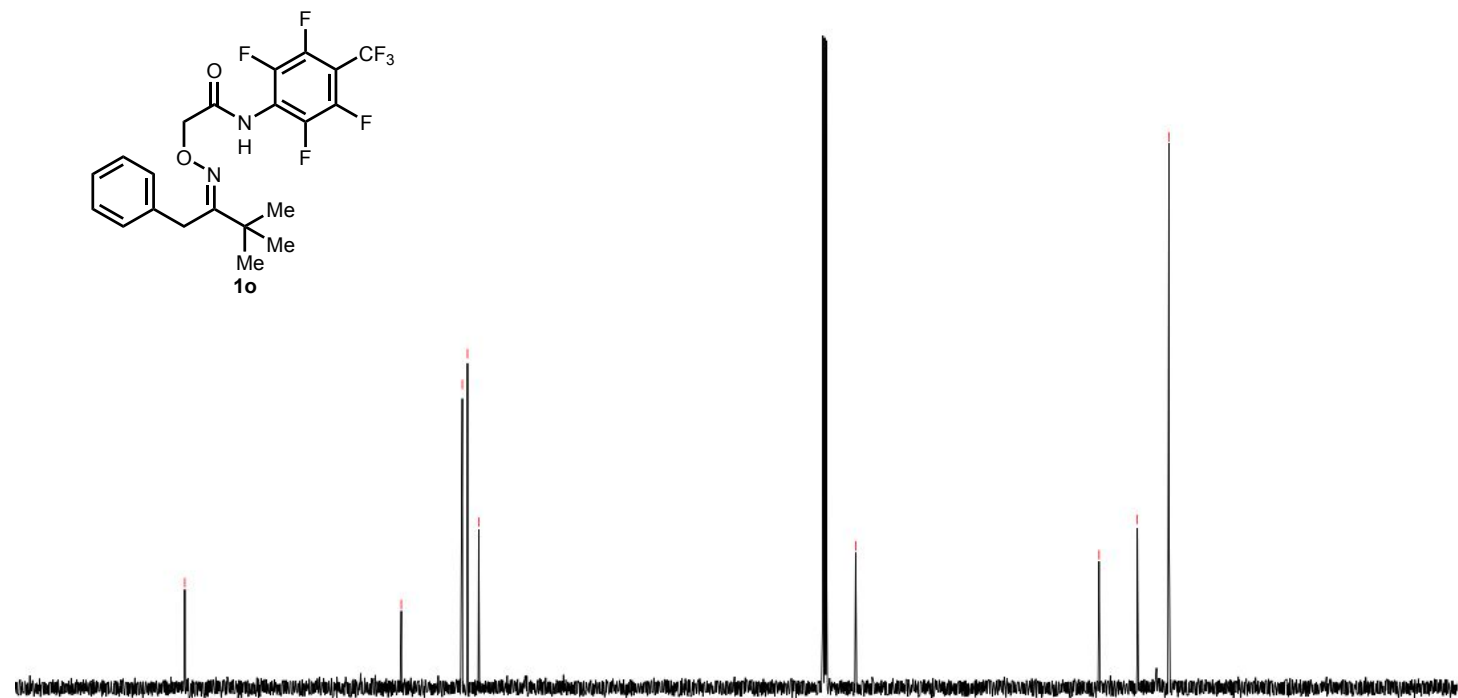

$\begin{array}{rlllllllllllllllllllllll}190 & 180 & 170 & 160 & 150 & 140 & 130 & 120 & 110 & 100 & 90 & 80 & 70 & 60 & 50 & 40 & 30 & 20 & 10 & 0 & -10\end{array}$ 

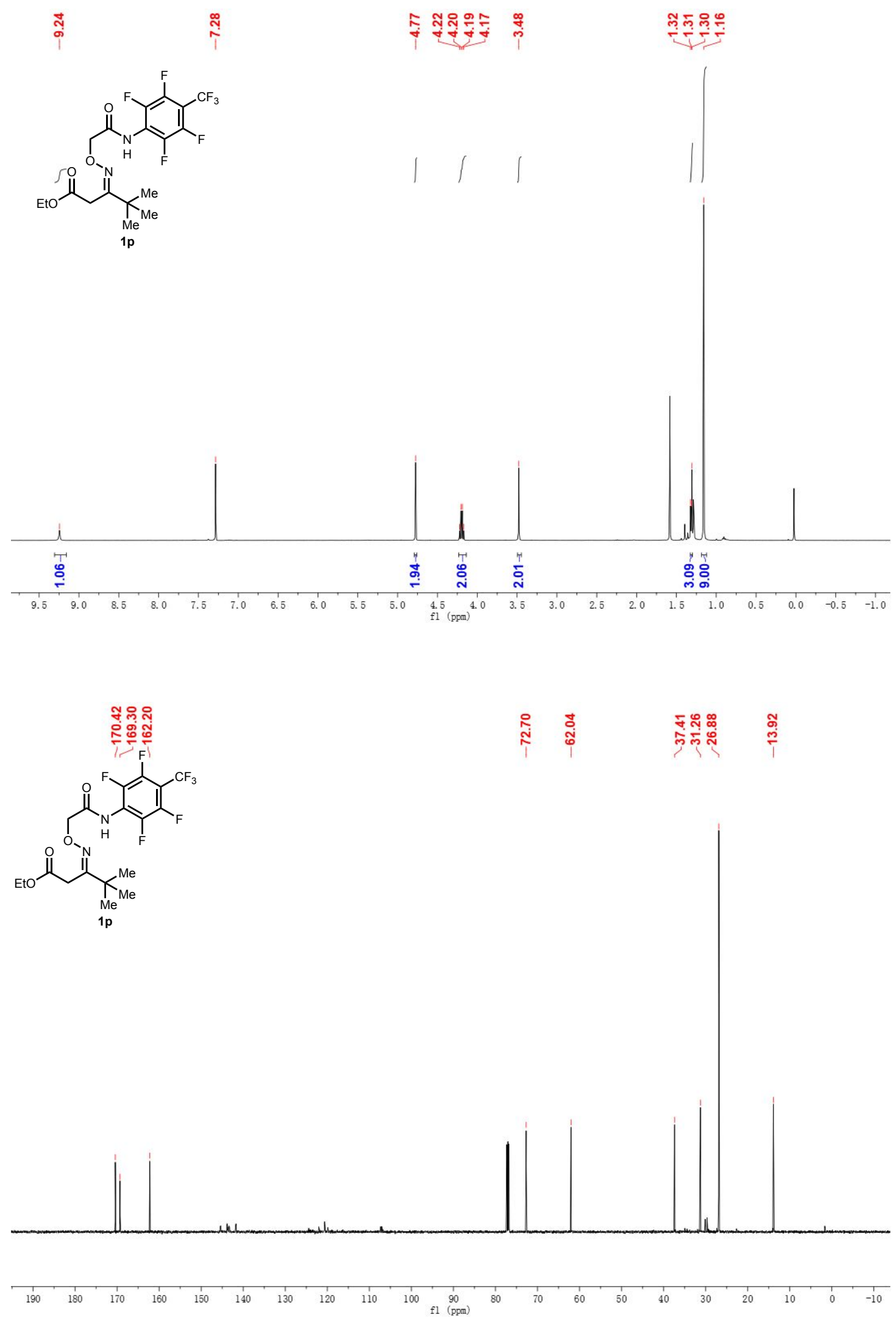


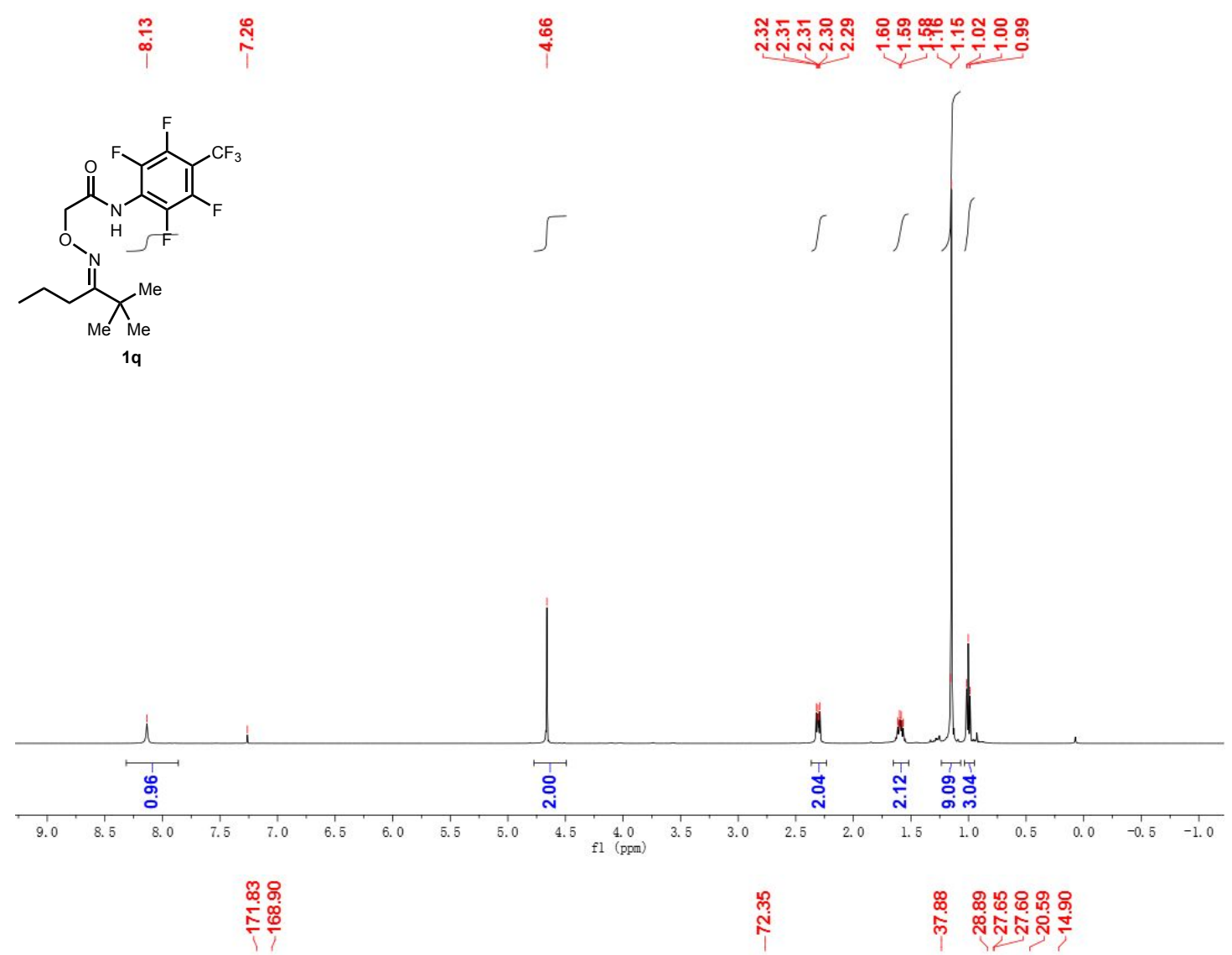<smiles>CCC/C(=N\OCC(=O)Nc1c(F)c(F)c(F)c(F)c1F)C(C)(C)C</smiles>

$1 q$

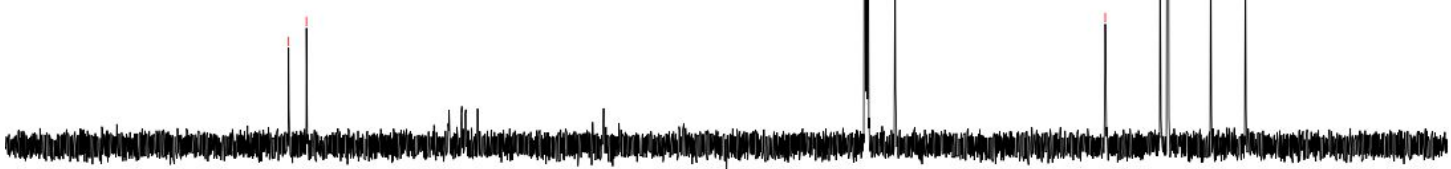

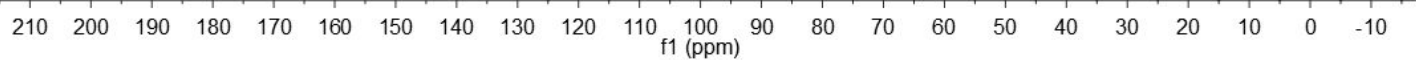



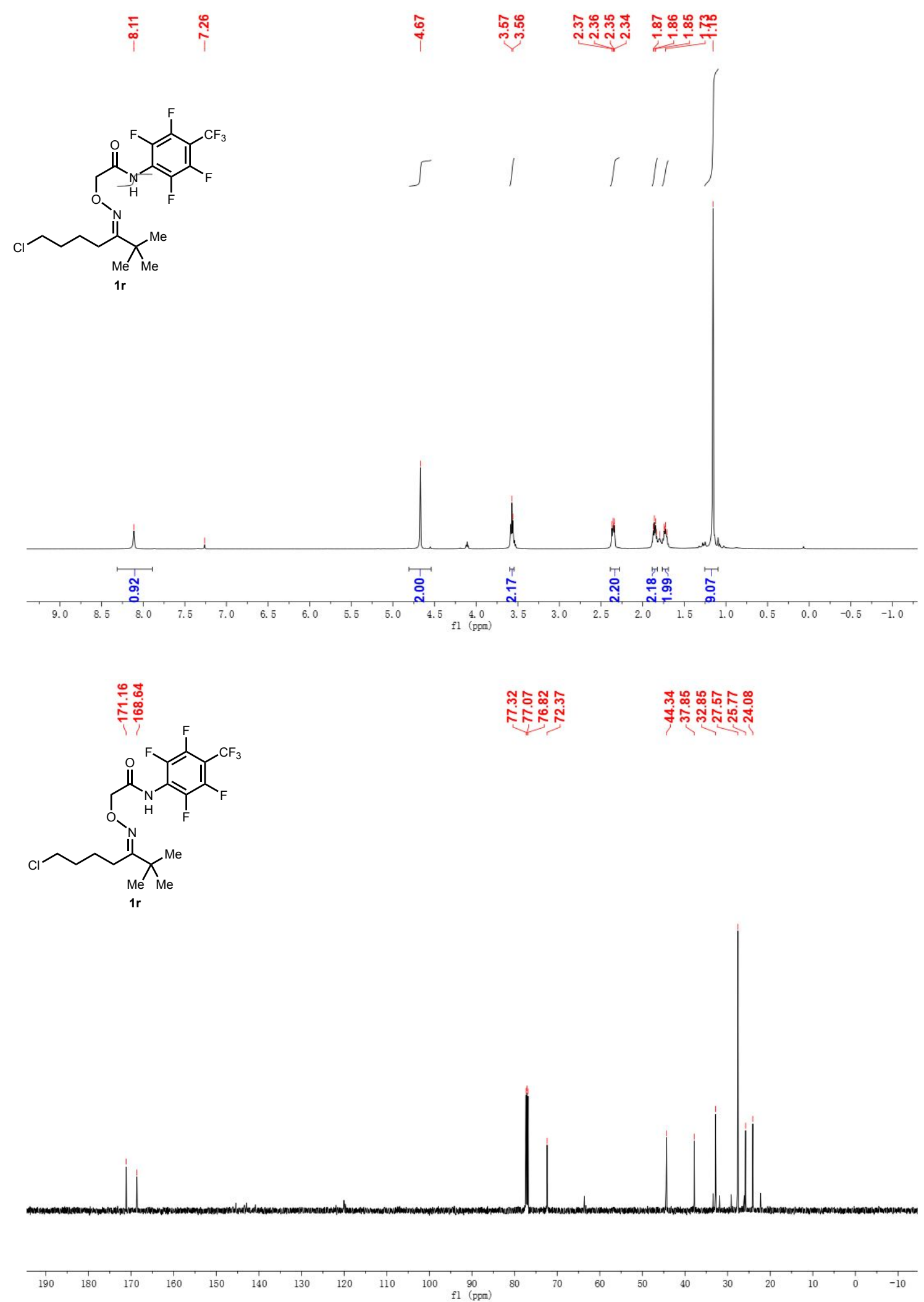
$\stackrel{\infty}{\infty} \stackrel{\substack{\infty \\ i}}{\uparrow}$

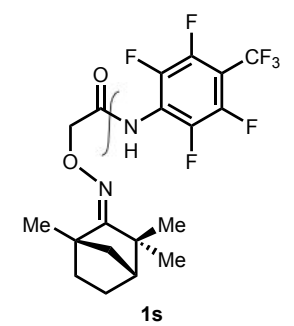

要

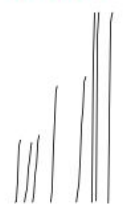

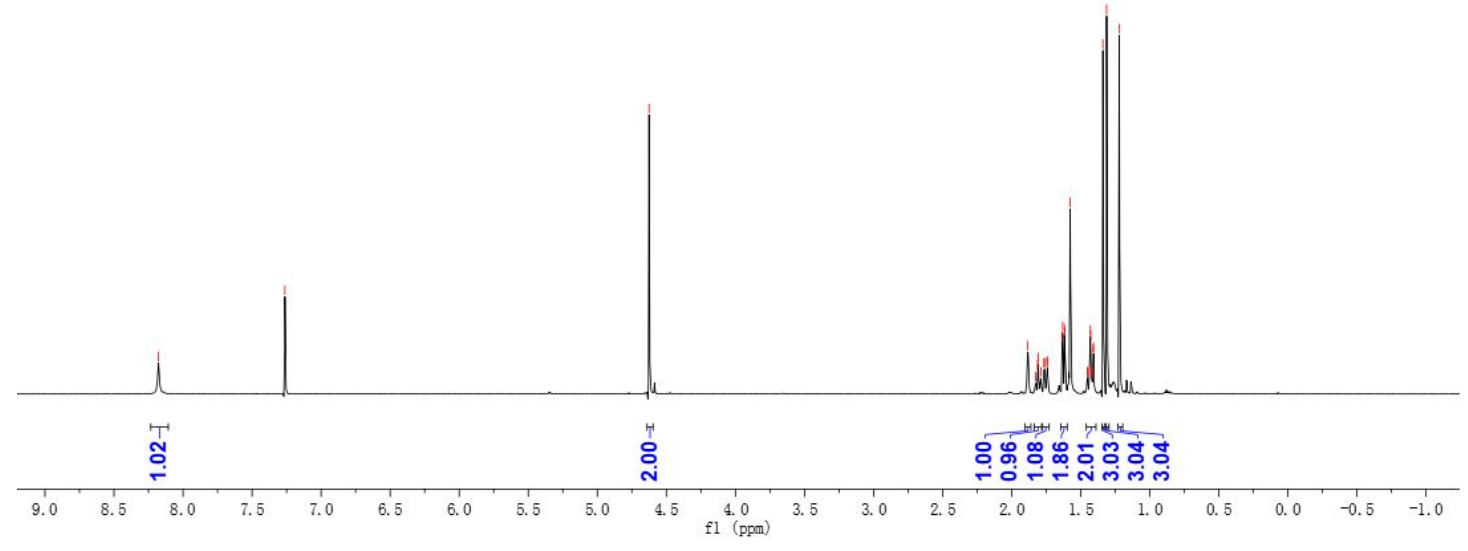

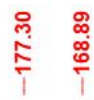<smiles>CC1(C)C(=NOCC(=O)Nc2c(F)c(F)c(F)c(F)c2F)[C@]2(C)CC[C@H]1C2(C)C</smiles>

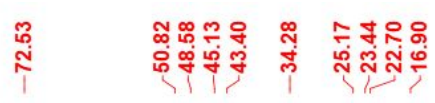

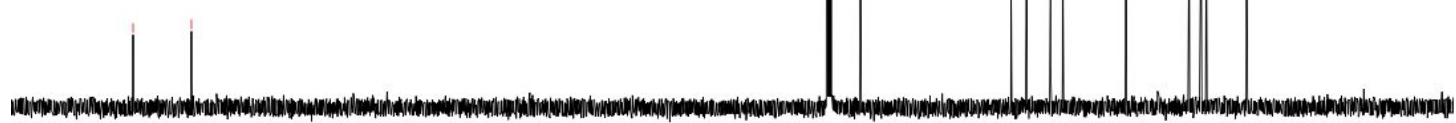

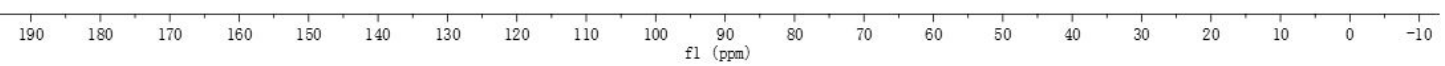



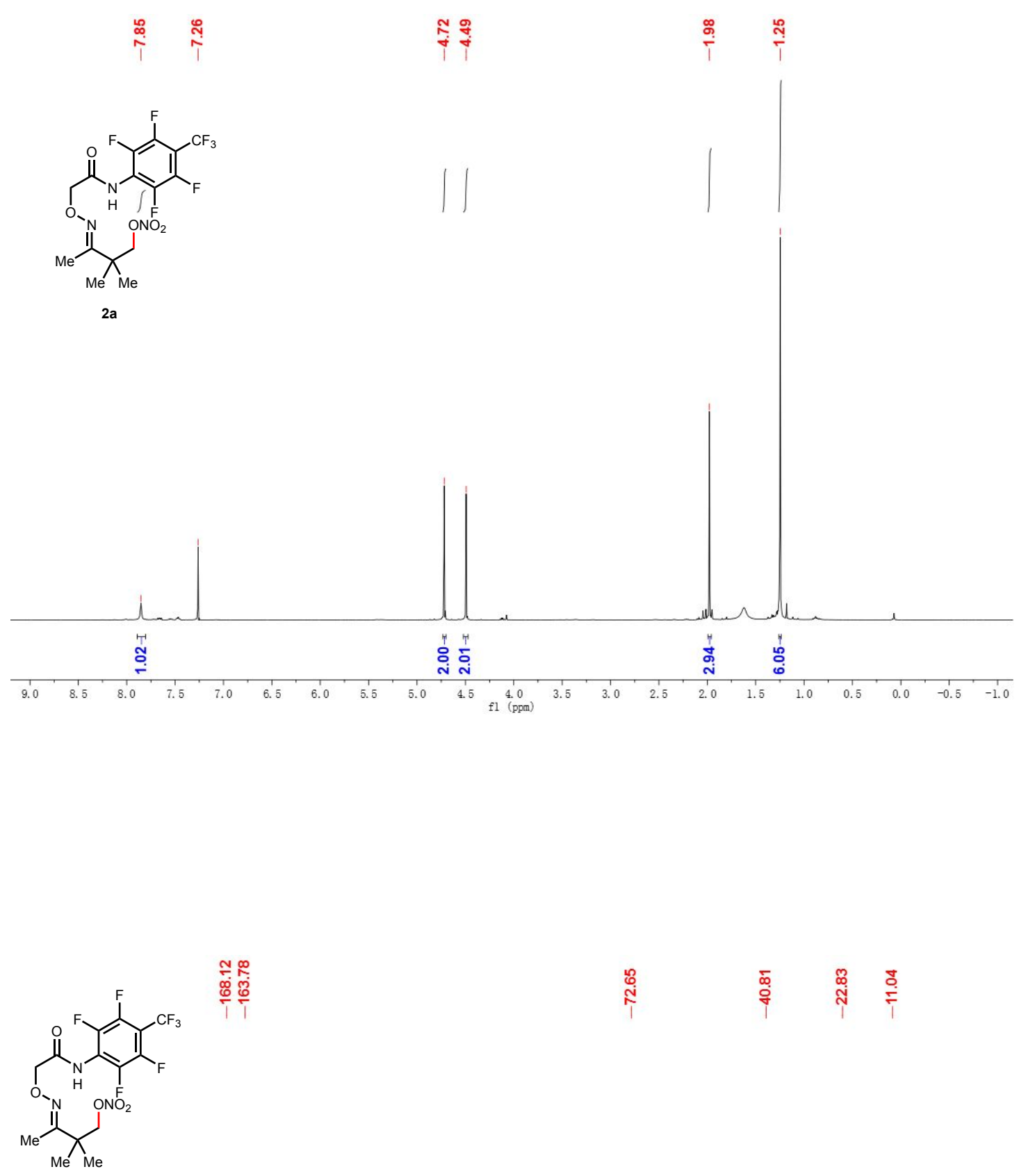

2a
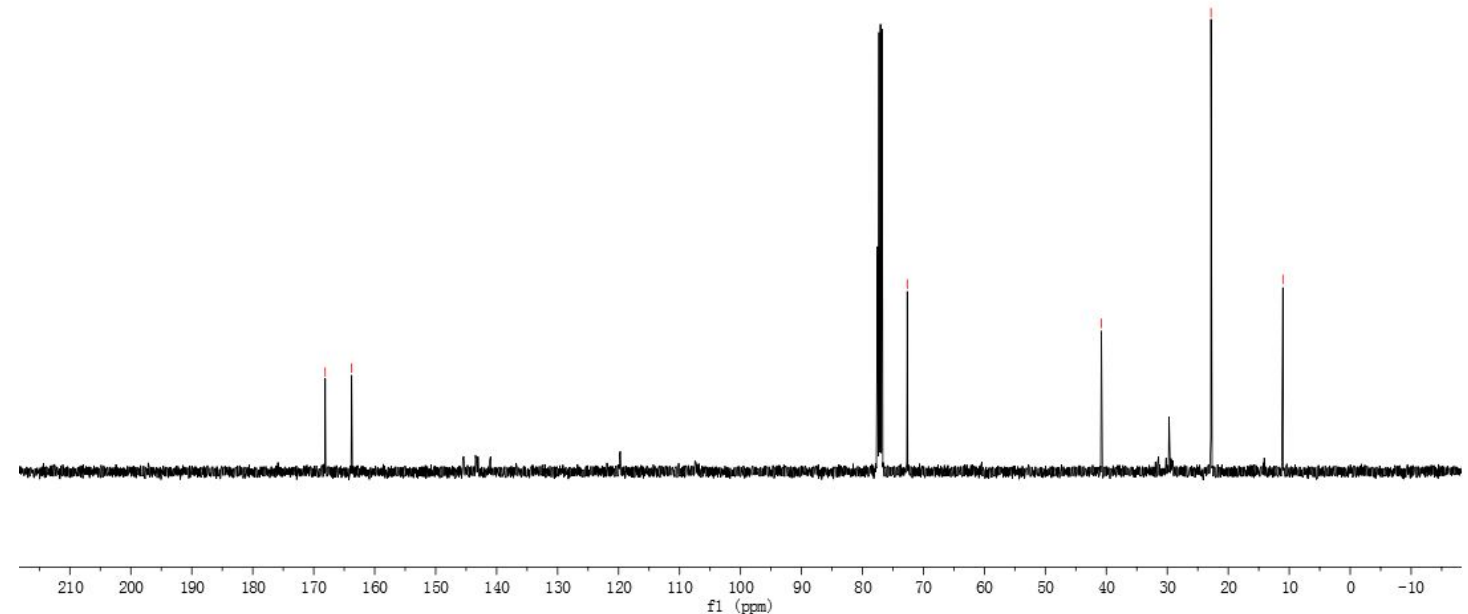

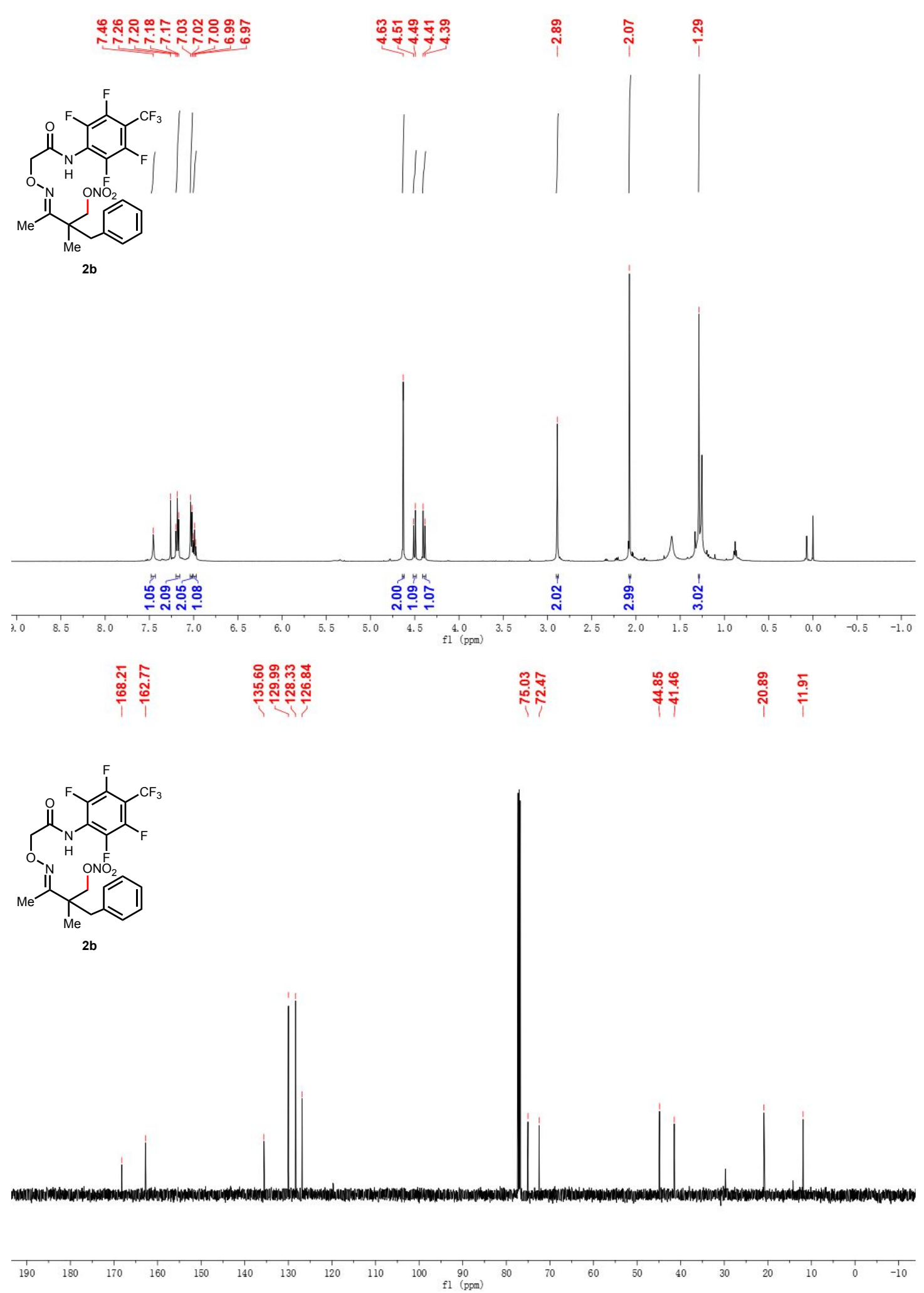

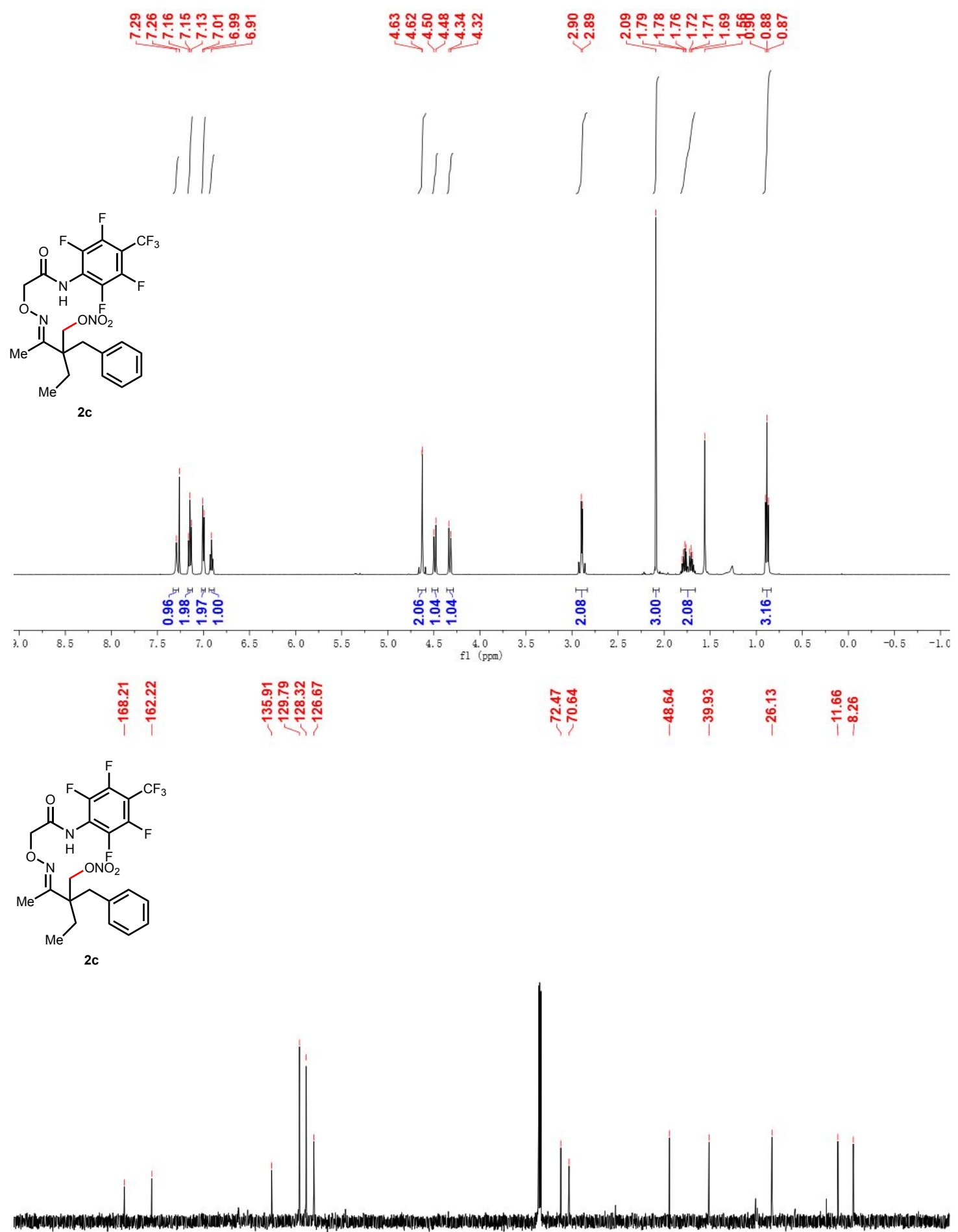

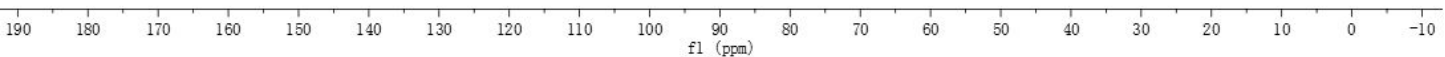



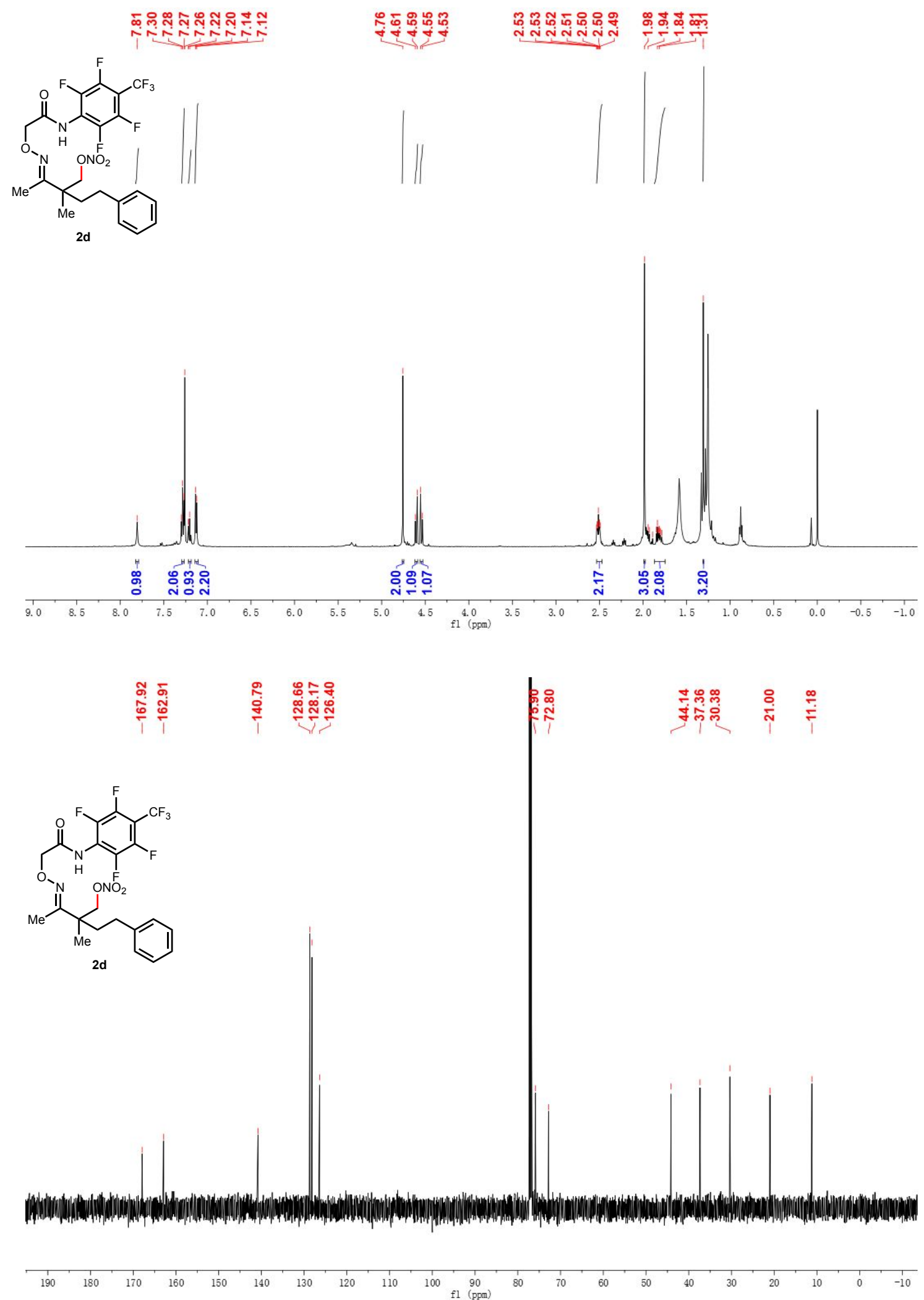


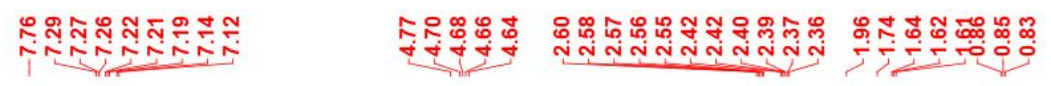
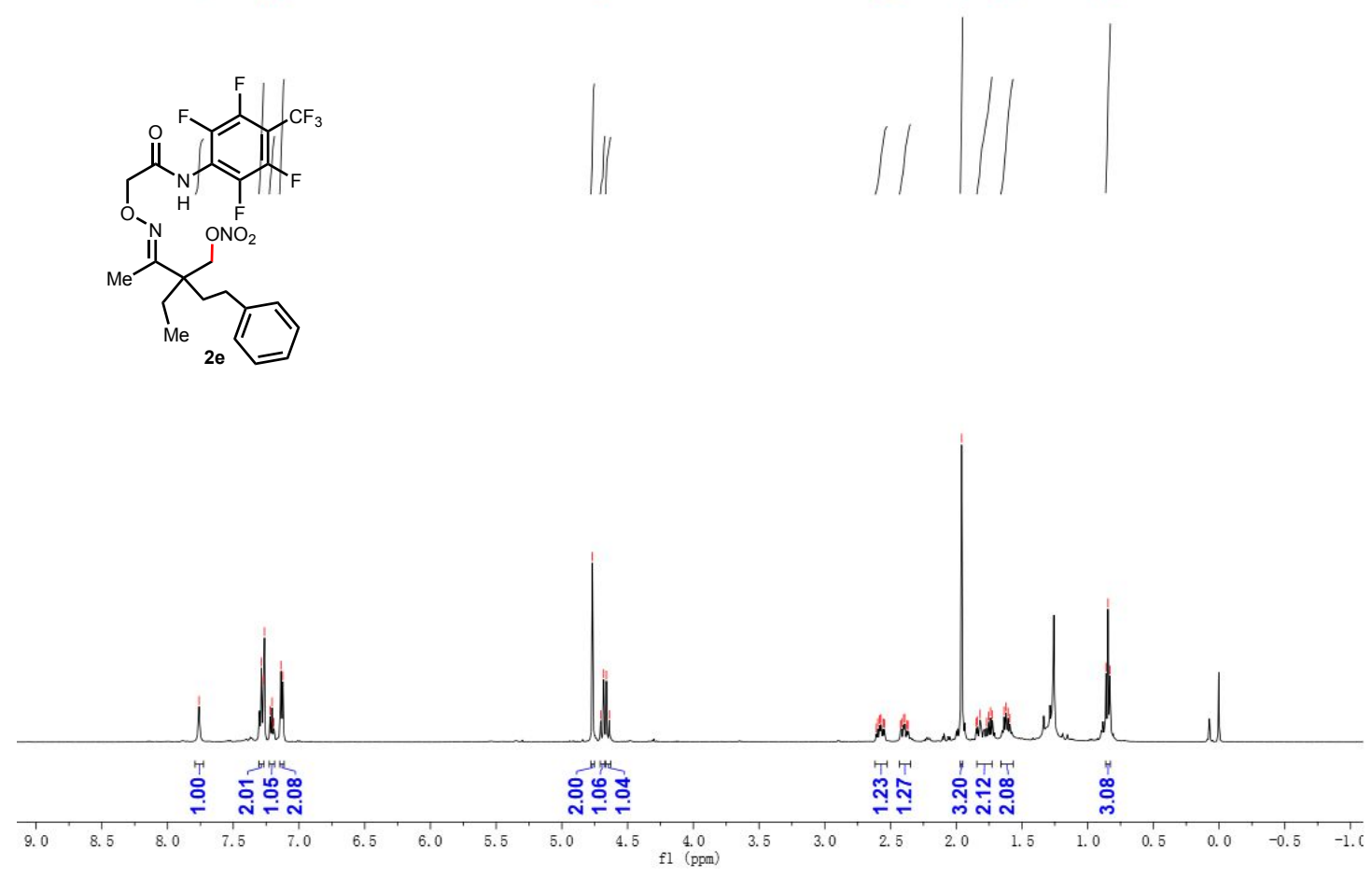
翌
官
交交

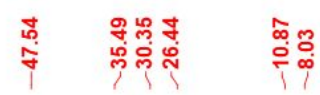

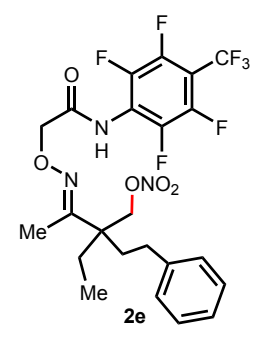

$\begin{array}{lllllllllll}190 & 180 & 170 & 160 & 150 & 140 & 130 & 120 & 110 & 100 & 90 \\ \mathrm{fl} & (\mathrm{ppm})\end{array}$

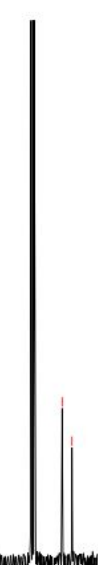




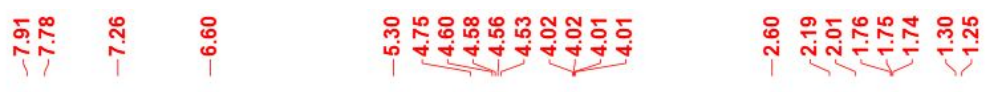

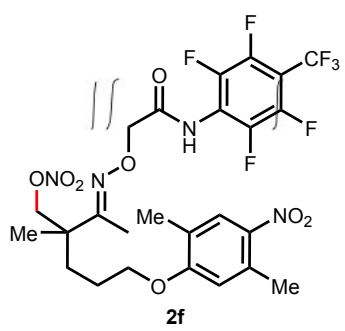<smiles>C=CC=C</smiles>
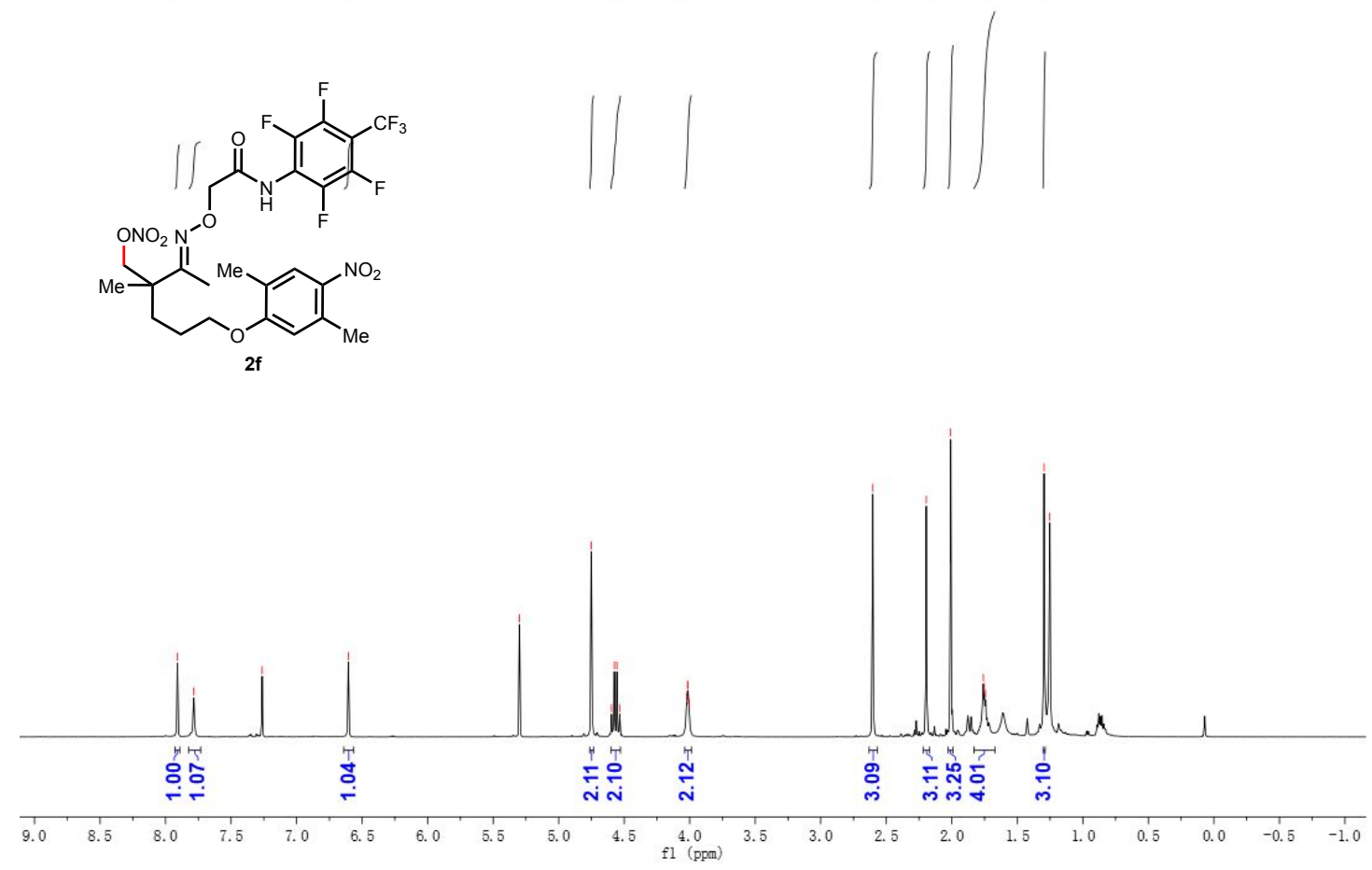
:
₹

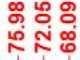
m

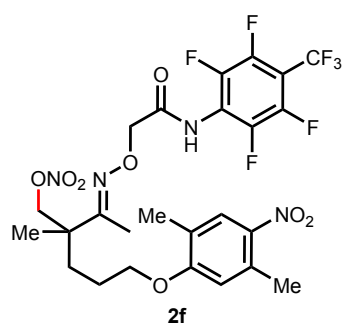

$\begin{array}{lllllllllll}190 & 180 & 170 & 160 & 150 & 140 & 130 & 120 & 110 & 100 & 90 \\ \mathrm{fl} & (\mathrm{ppm})\end{array}$ 

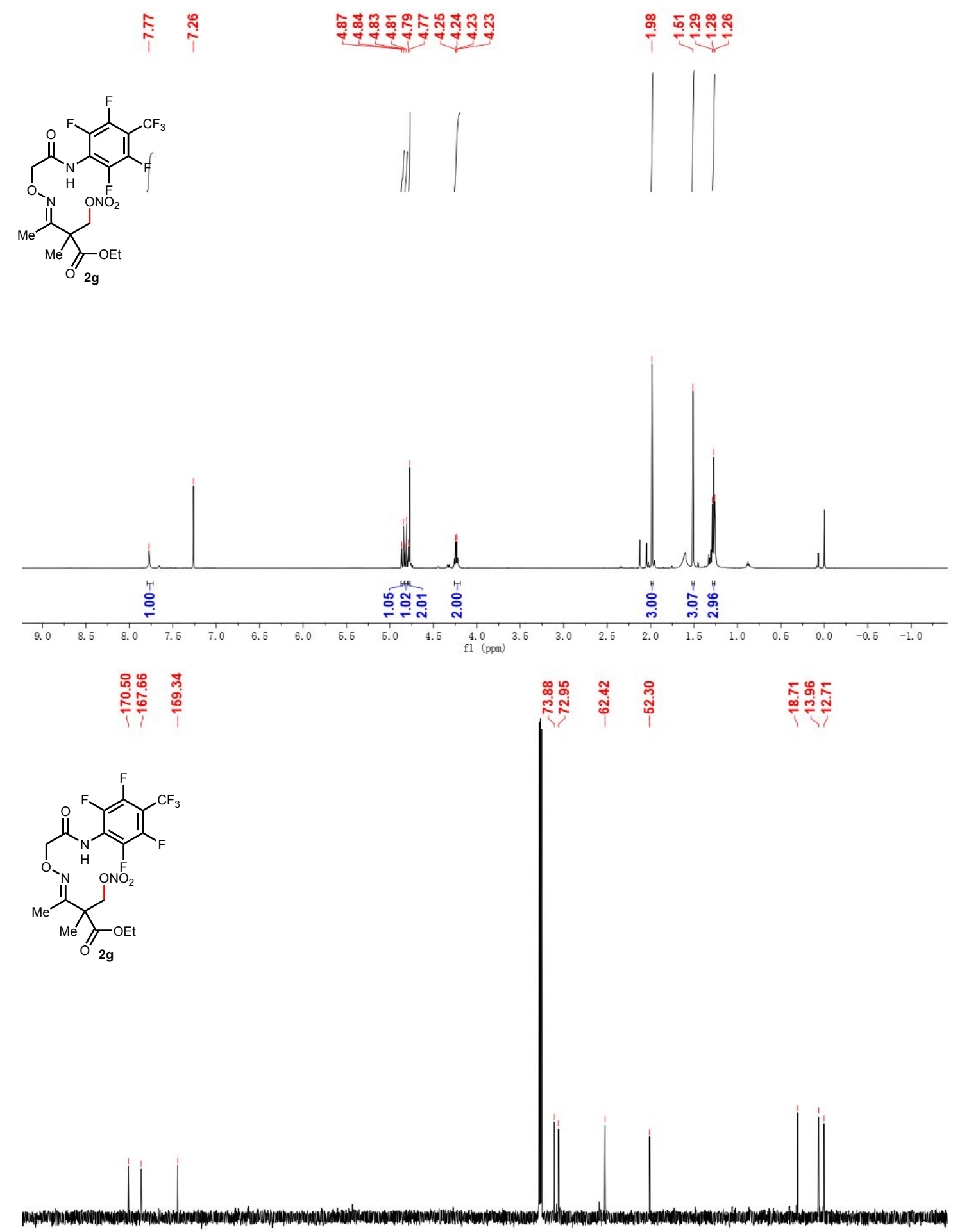

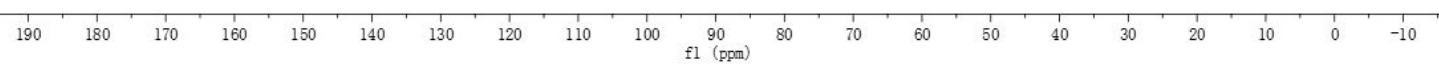



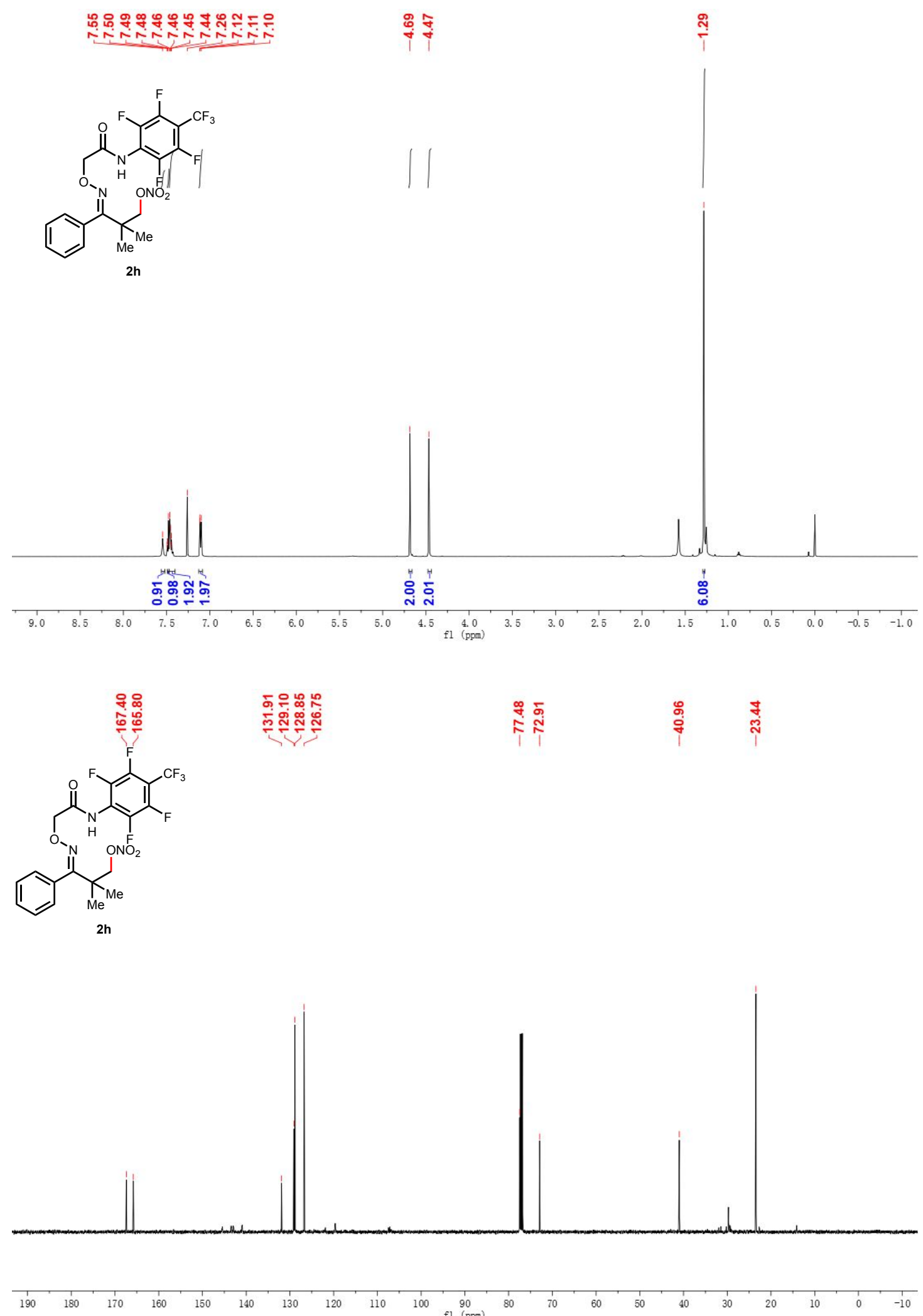

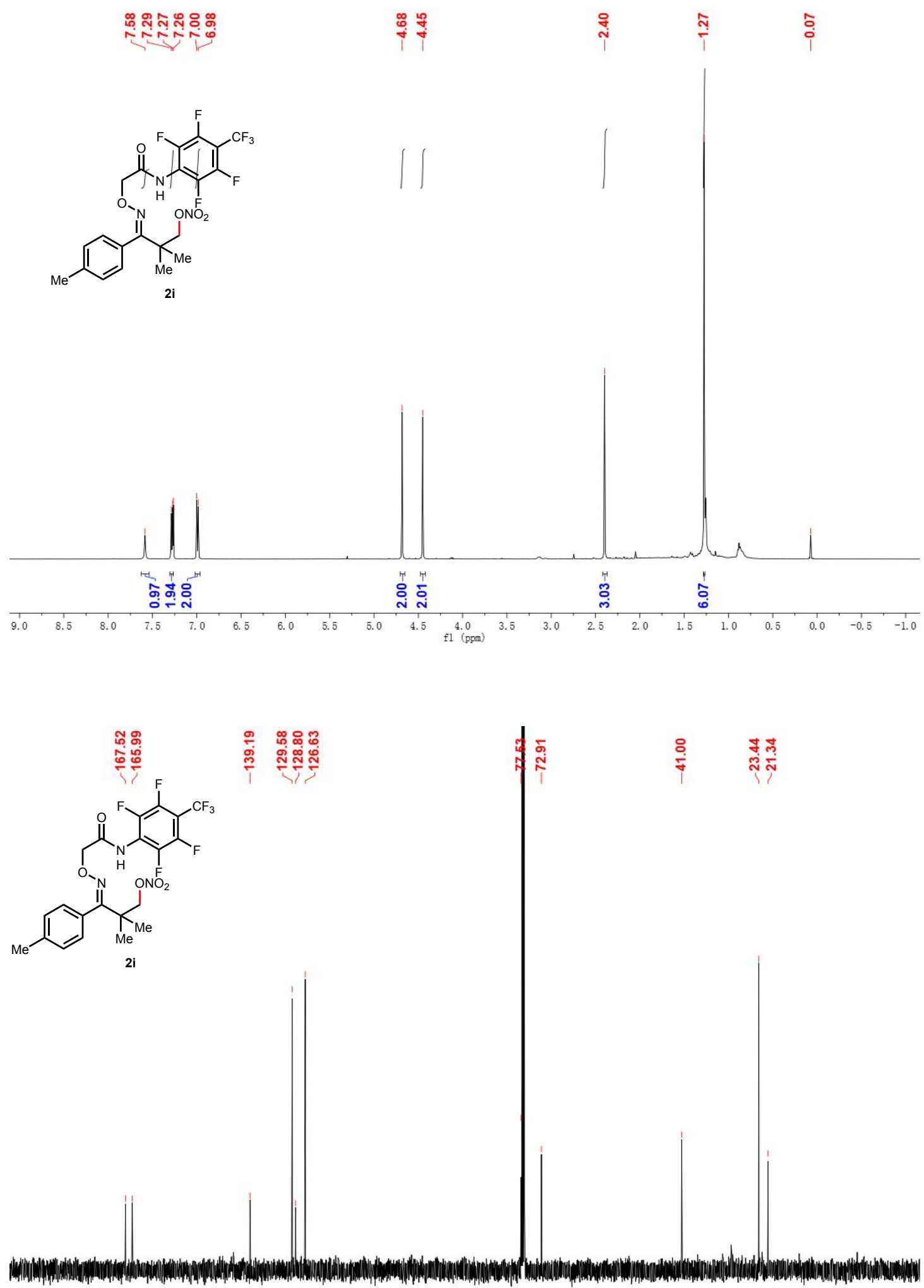

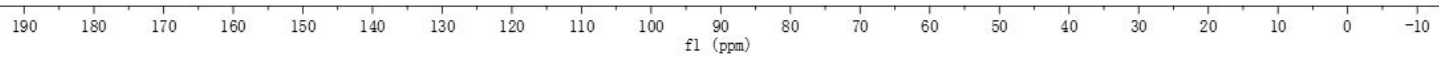



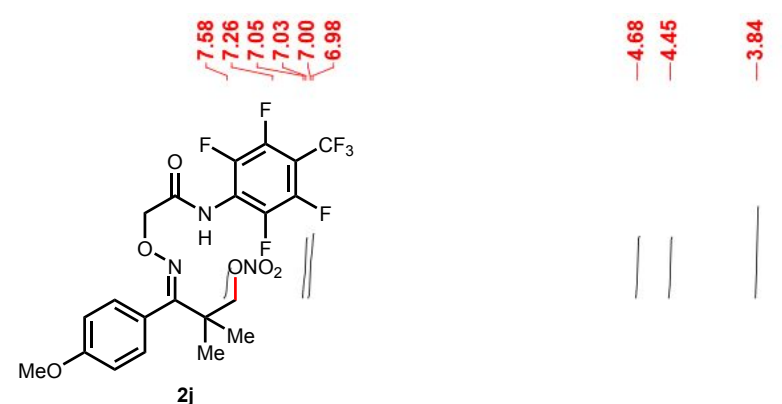

$\stackrel{\text { Ț }}{i}$
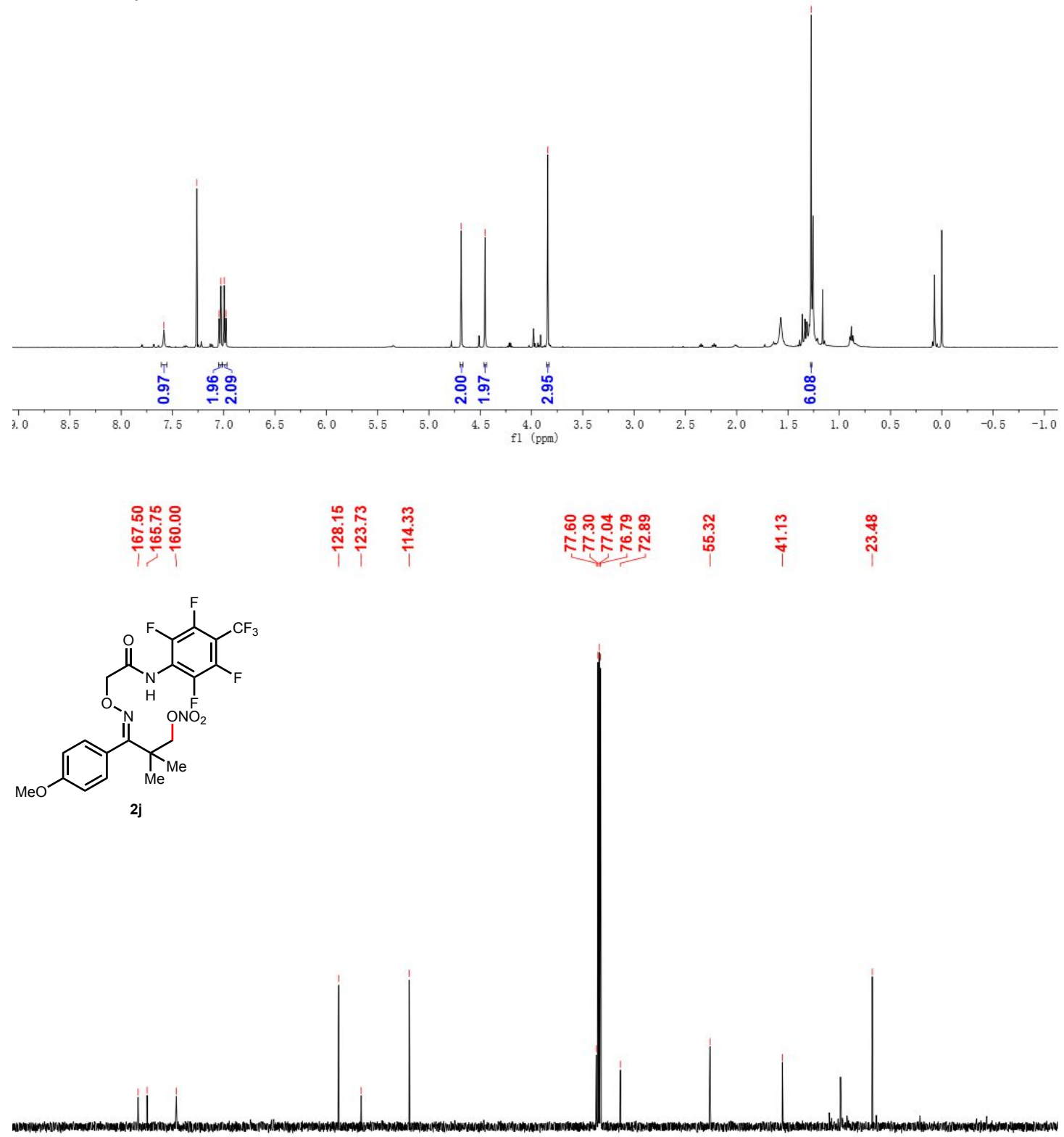

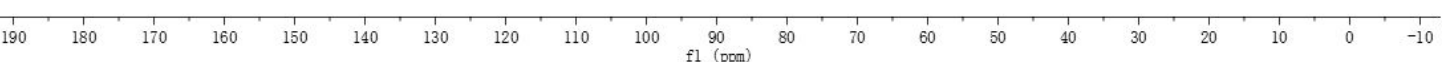



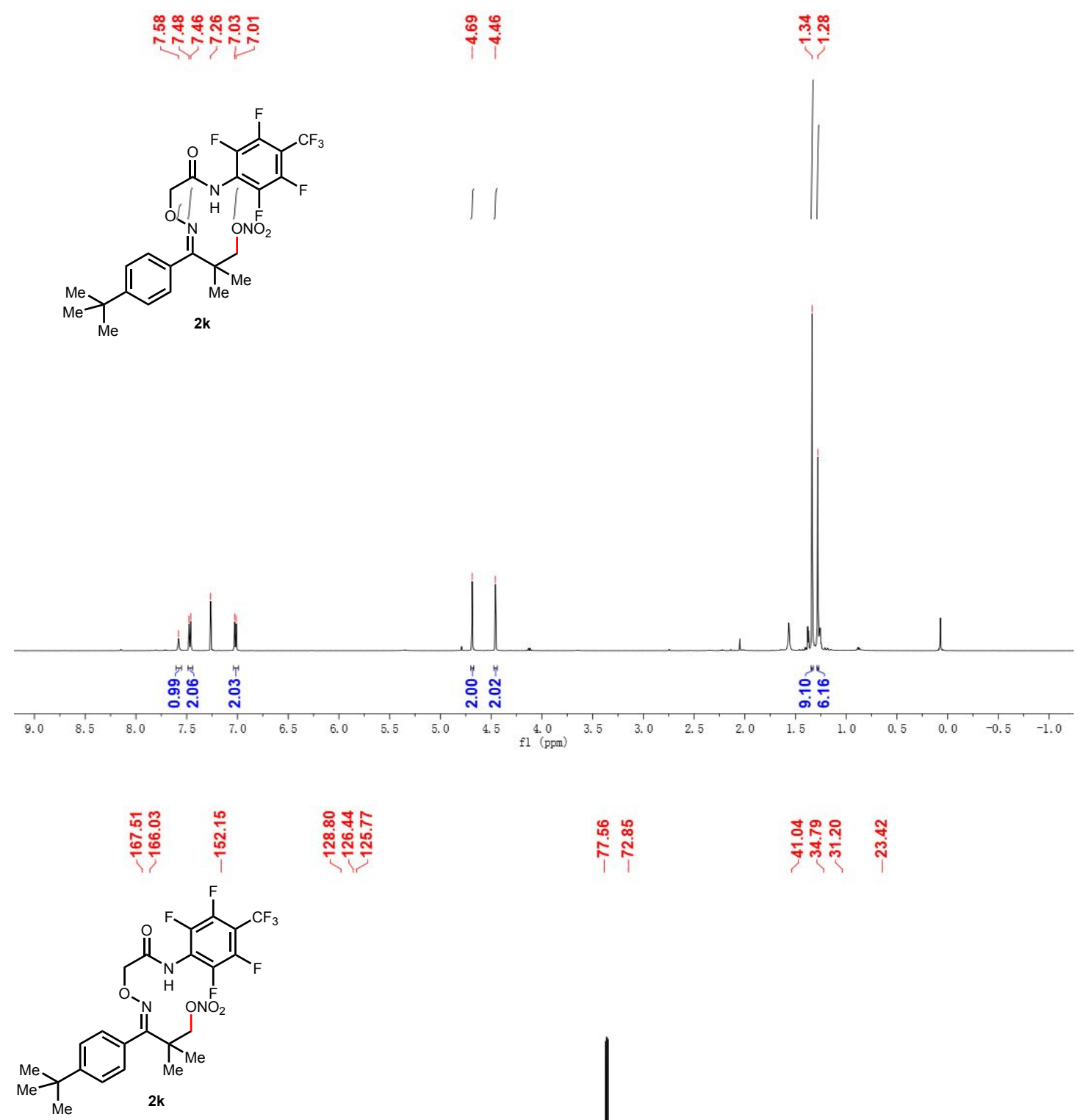

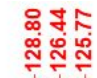

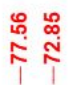

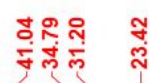

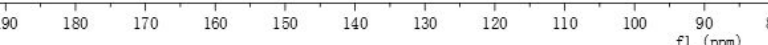



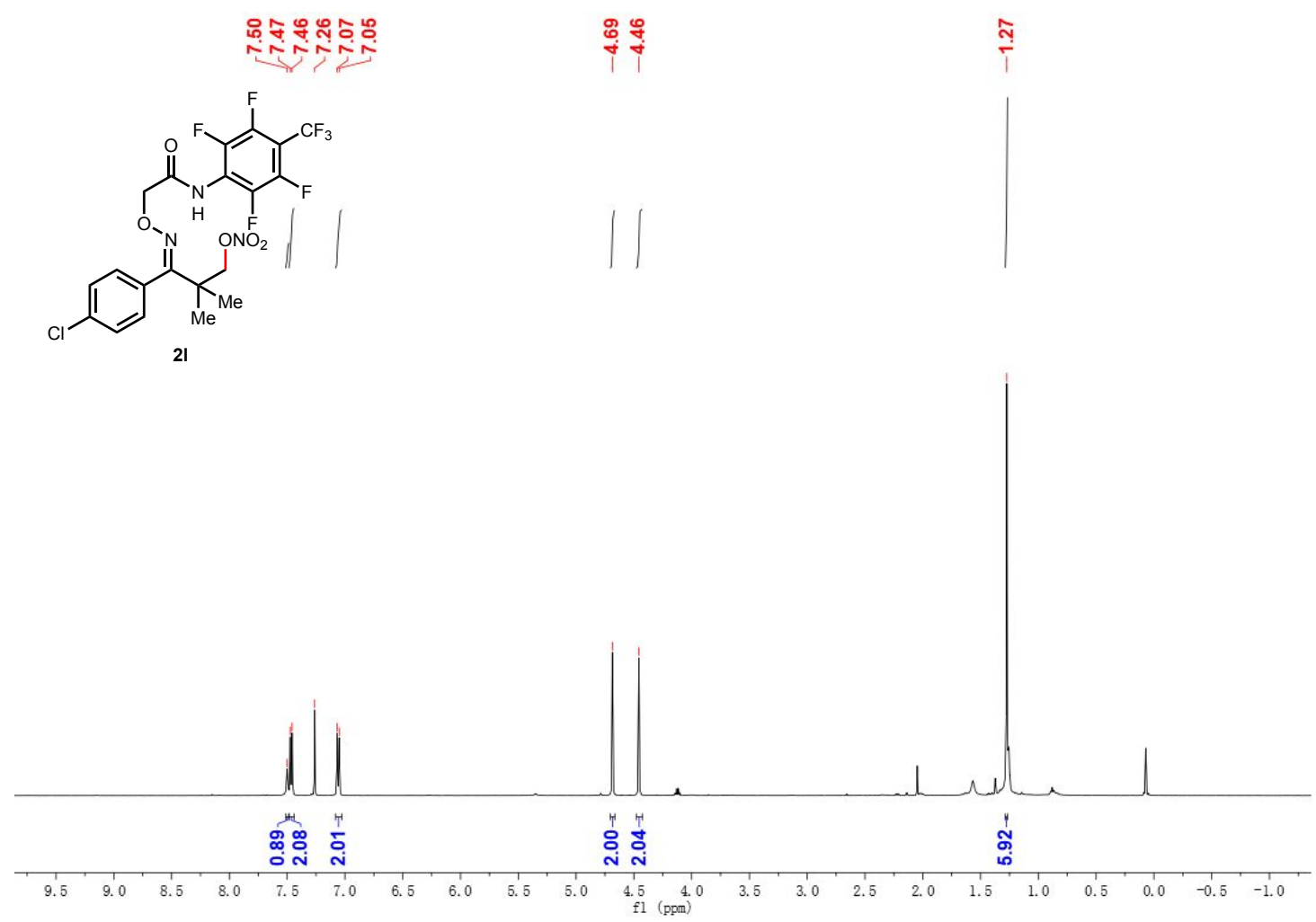<smiles>CC(C)(CO)C(=NOCC(=O)Nc1c(F)c(F)c(C(F)(F)F)c(F)c1[N+](=O)[O-])c1ccc(Cl)cc1</smiles>

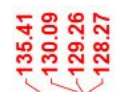

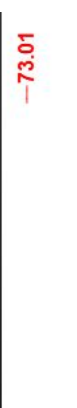

ஓे

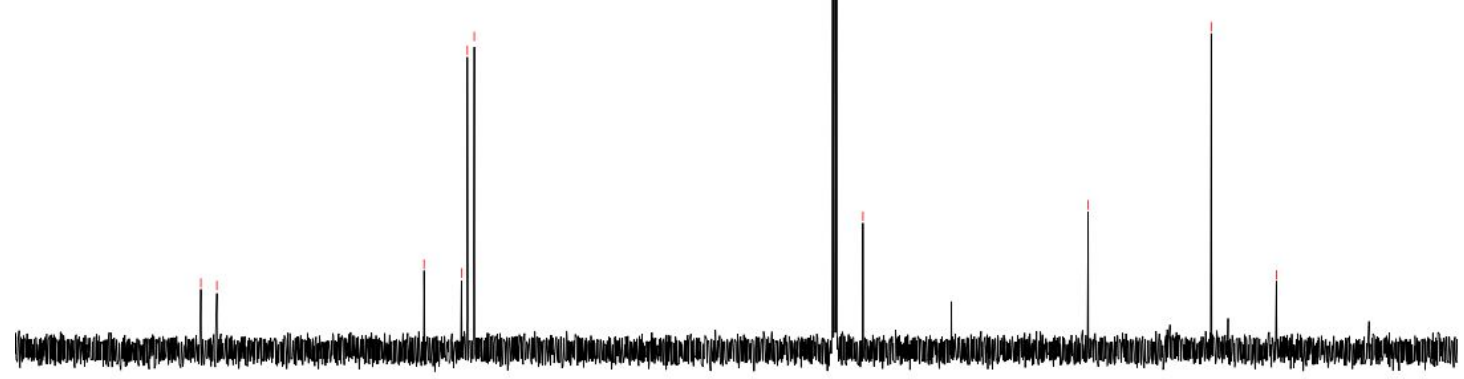

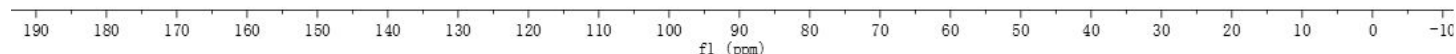



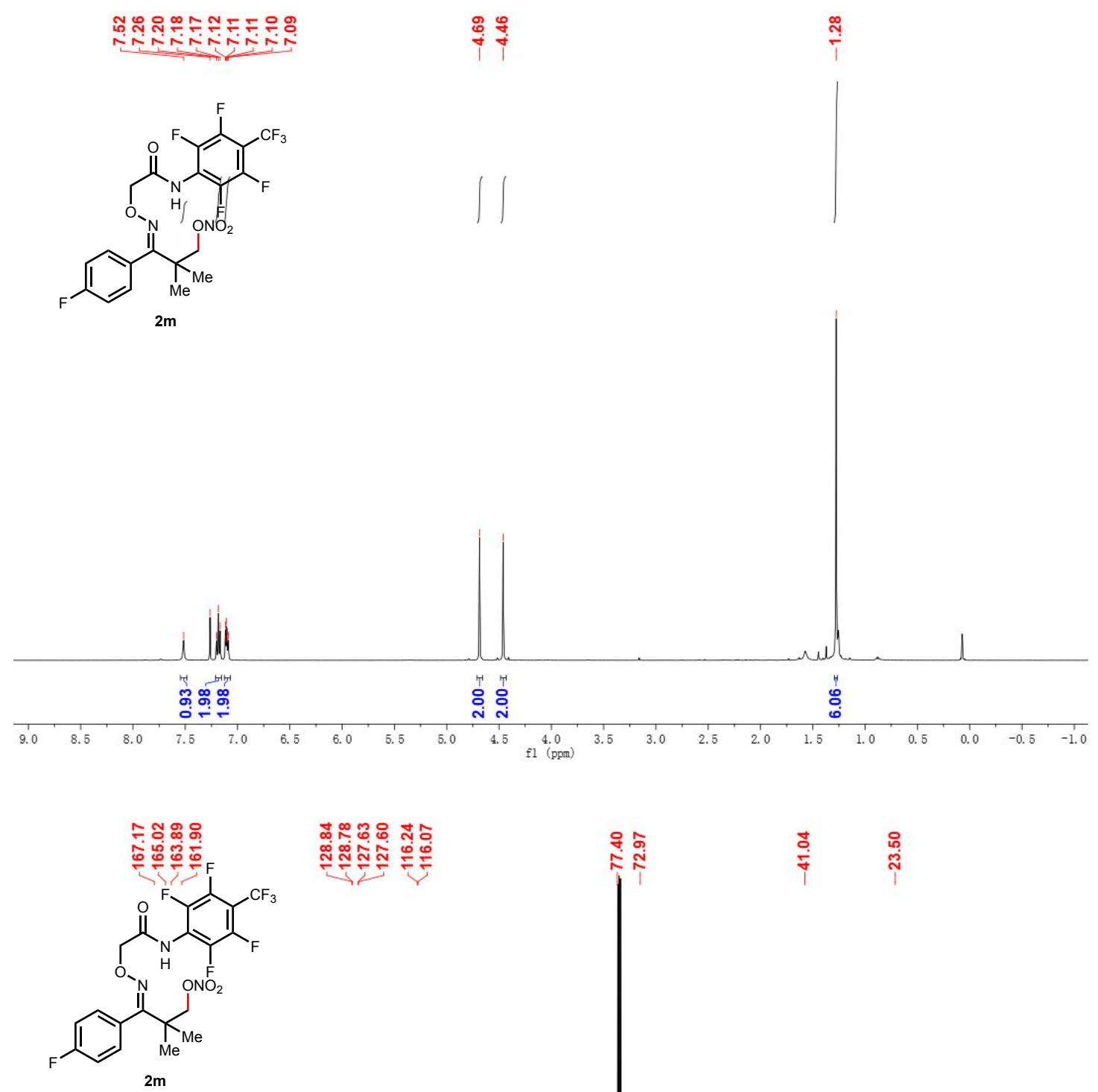

\section{广ே}

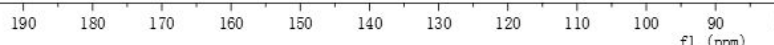

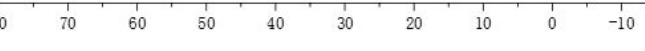



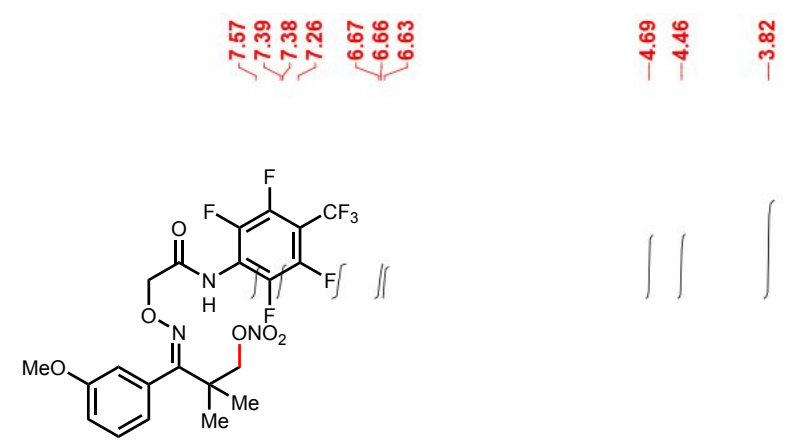

꾸

2n
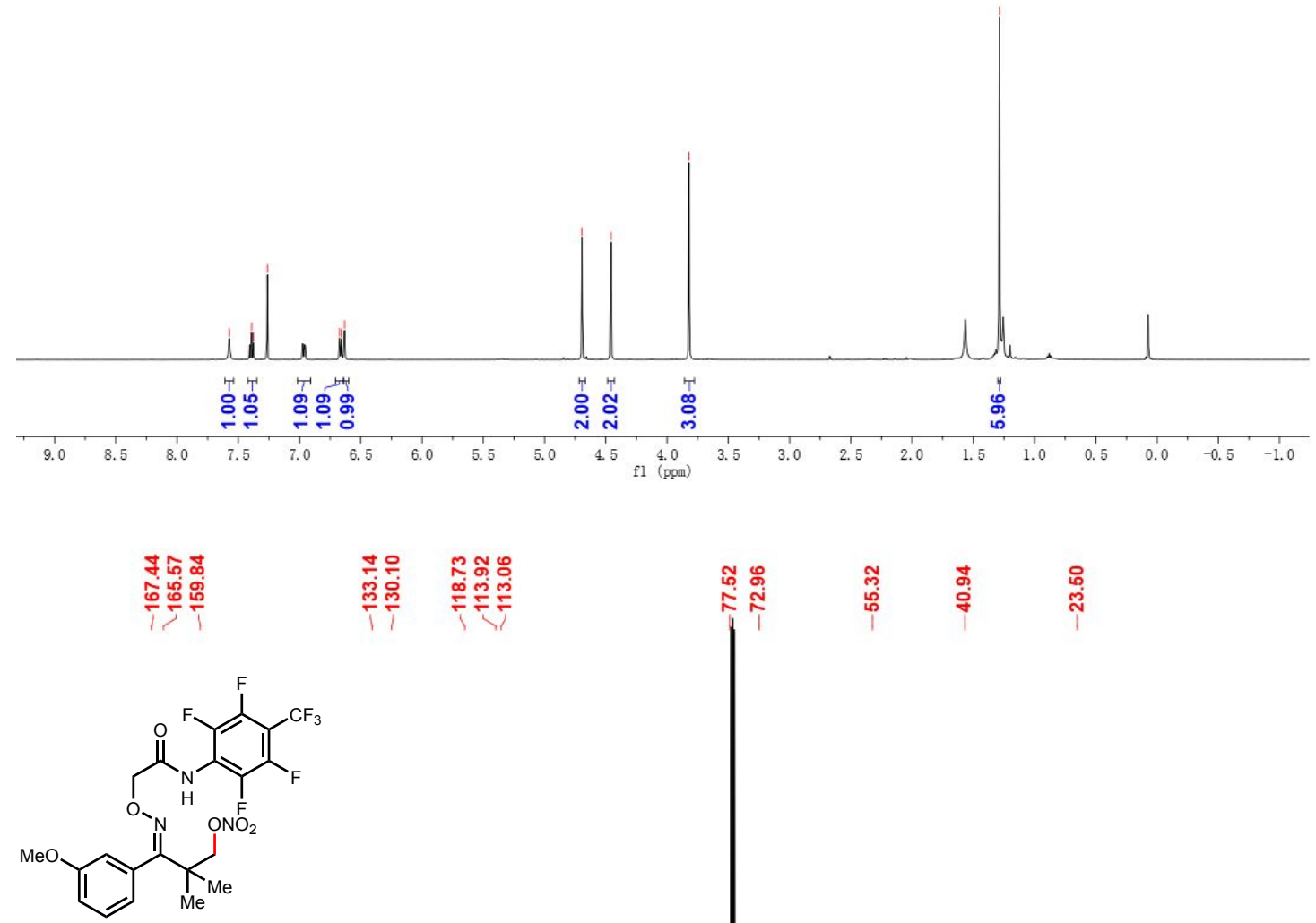

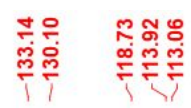

กิ

2n
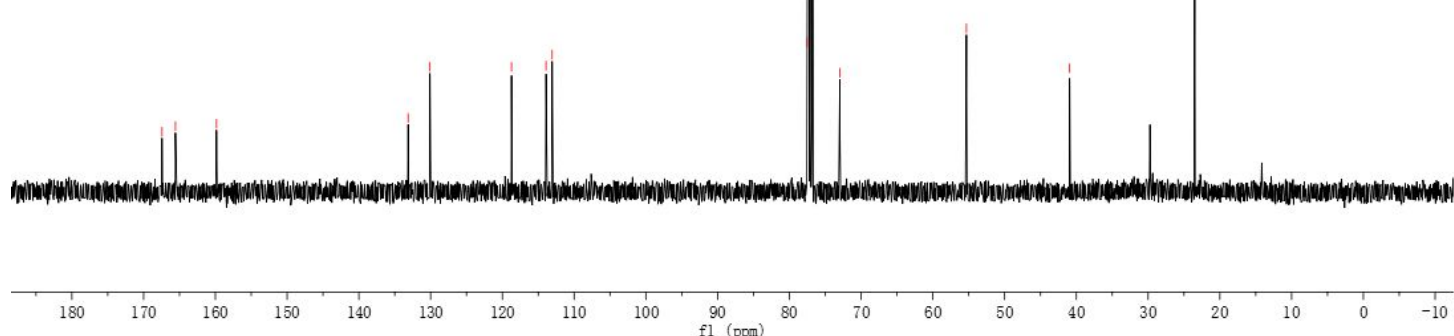

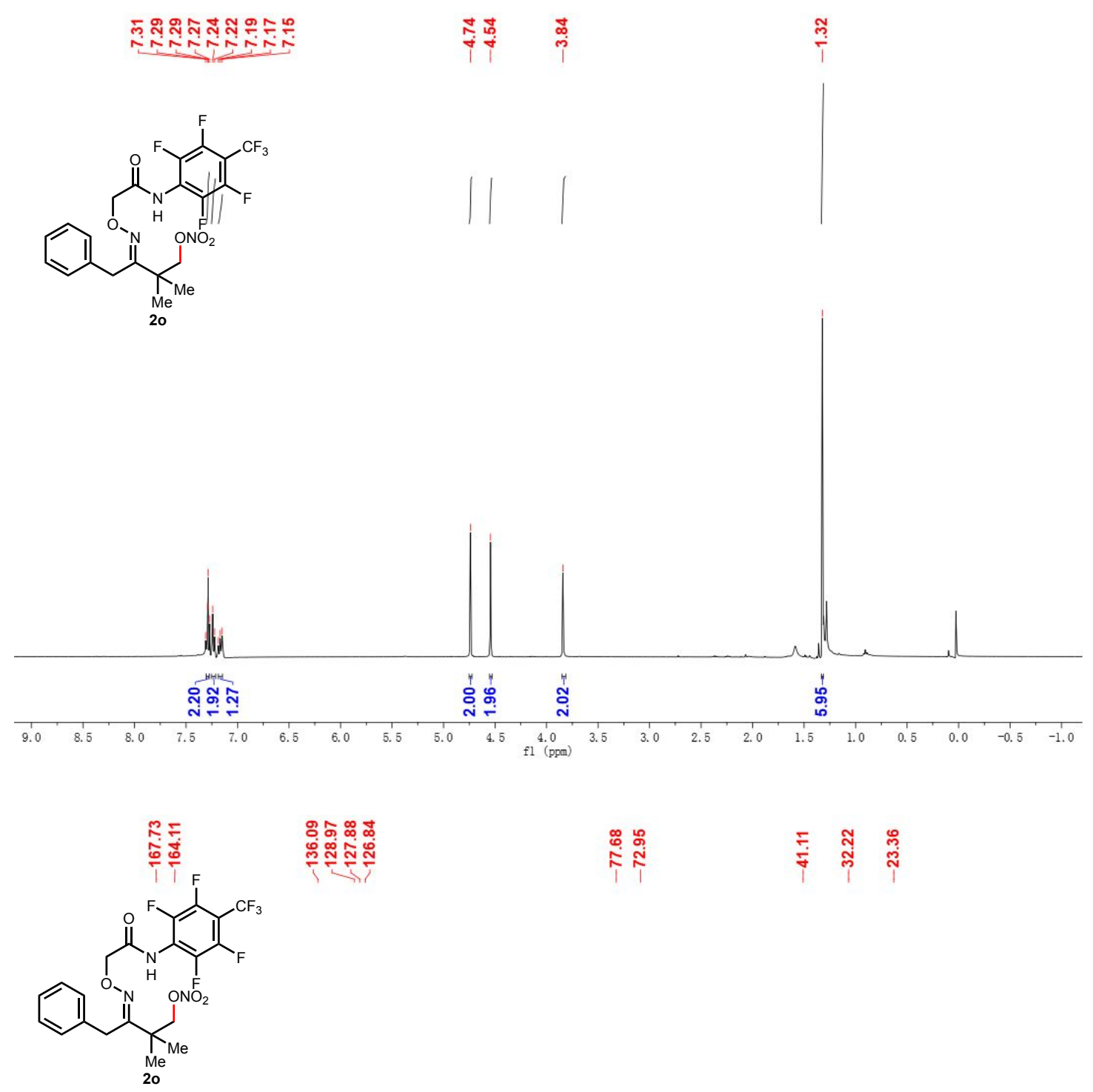

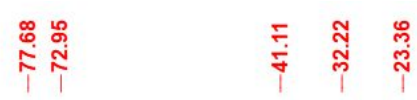

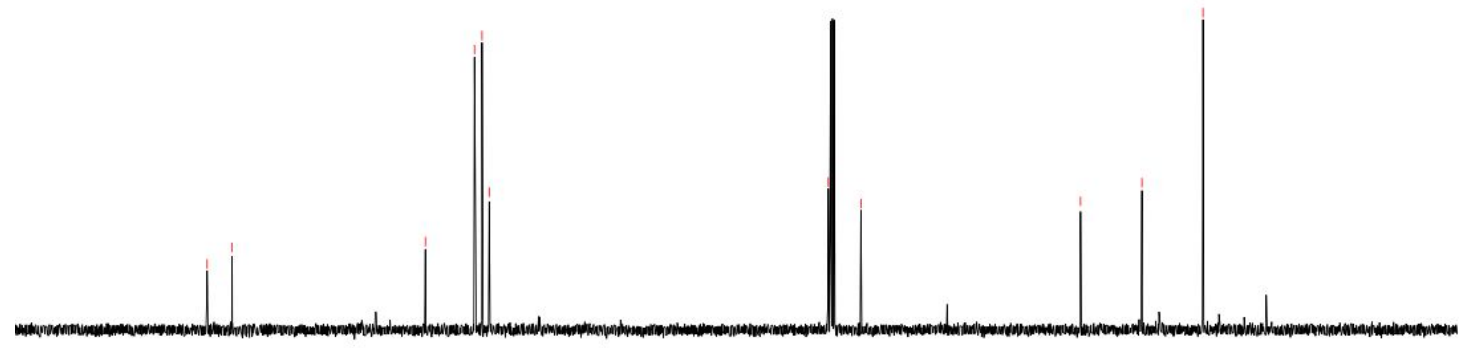

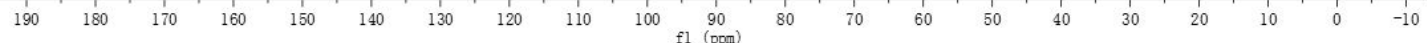




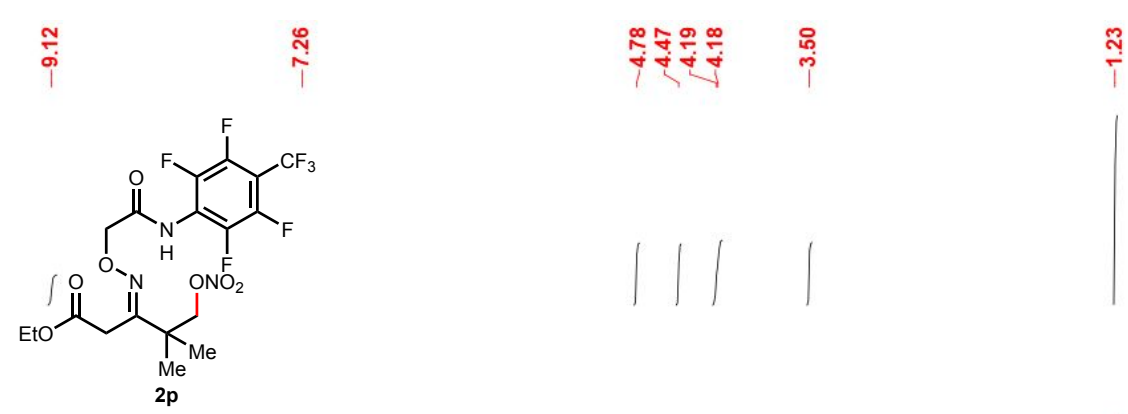

$\stackrel{i}{\stackrel{1}{\pi}}$

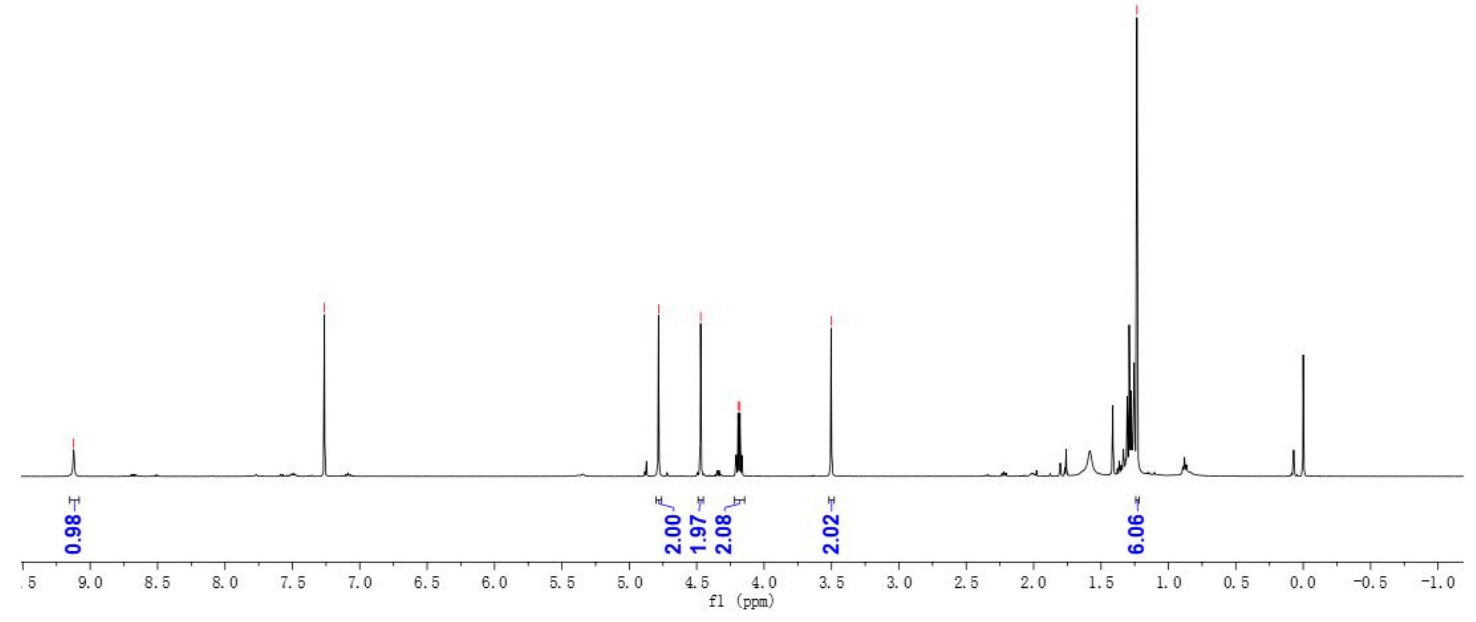

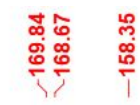<smiles>CC(C)(C)C(CC(=O)O)C(C)(C)C</smiles>

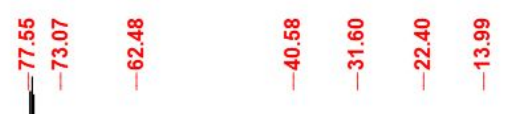

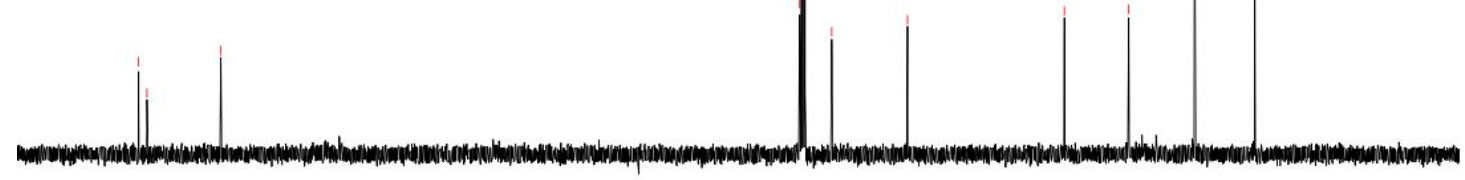

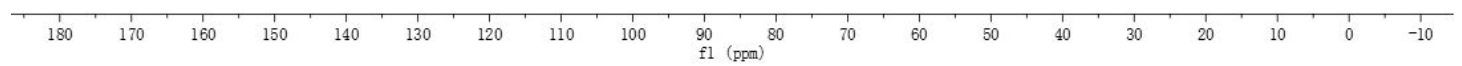



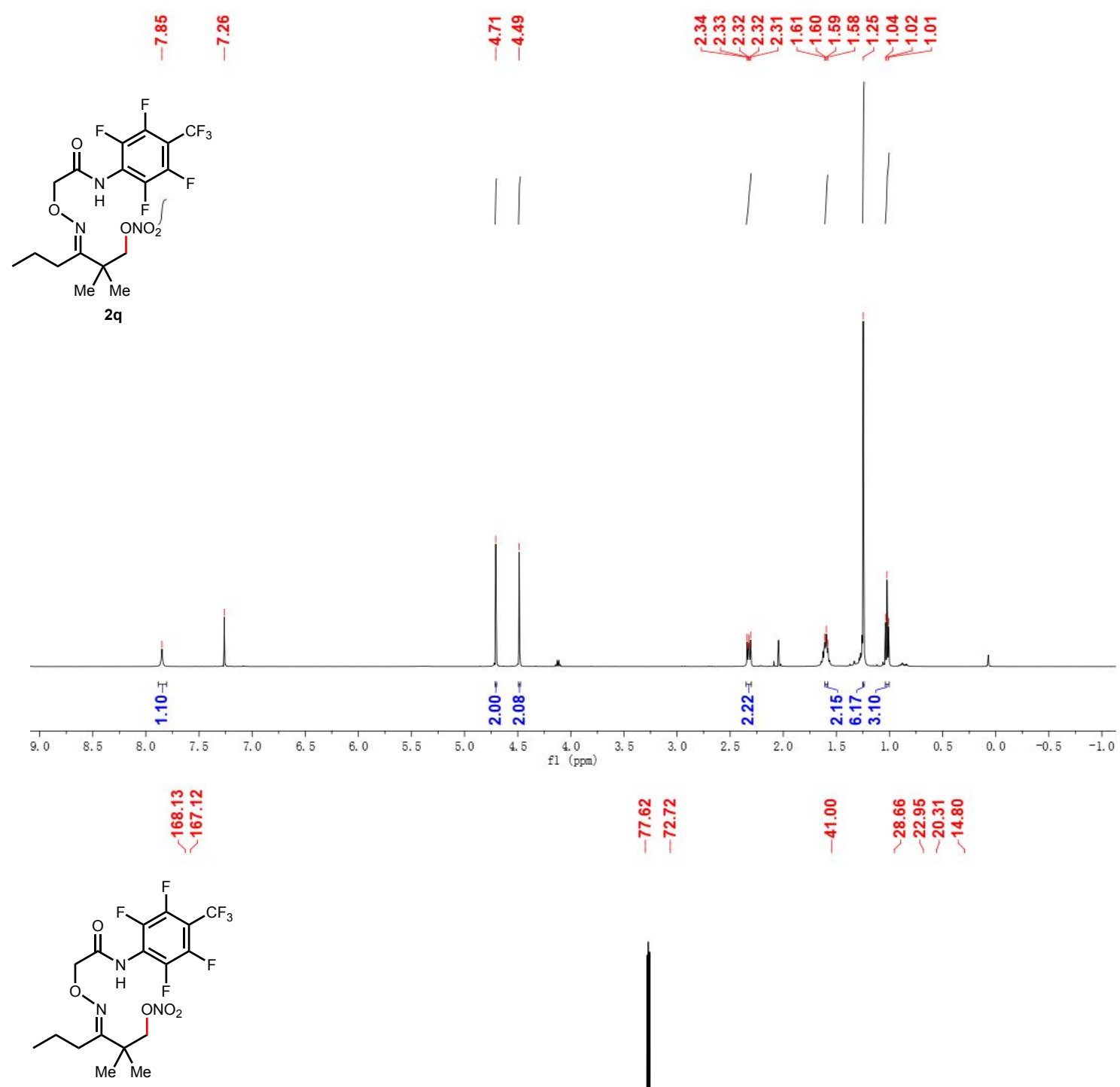

$2 q$

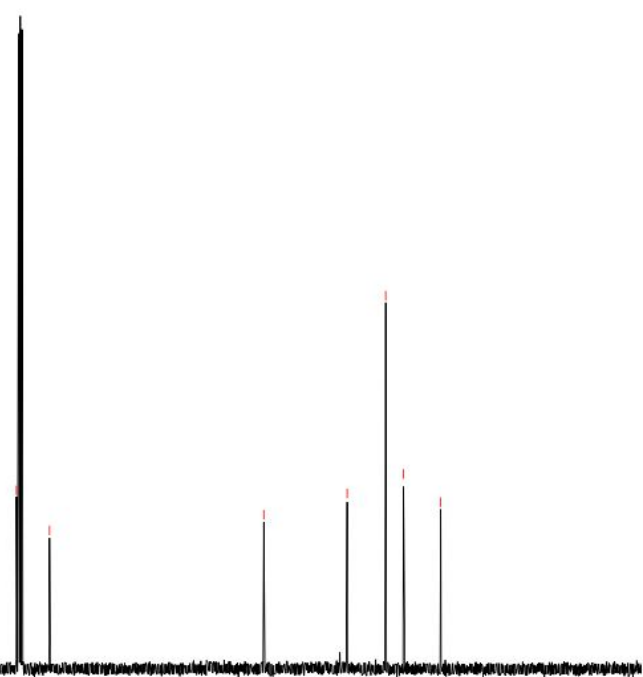

\begin{tabular}{llllllllllllllllllllll}
1 & 1 \\
190 & 180 & 170 & 160 & 150 & 140 & 130 & 120 & 110 & 100 & 1 & 1 & 1 & 1 & 1 & 1 & 1 & 1 & 1 & 1 & 1 & 1 \\
\hline
\end{tabular} 


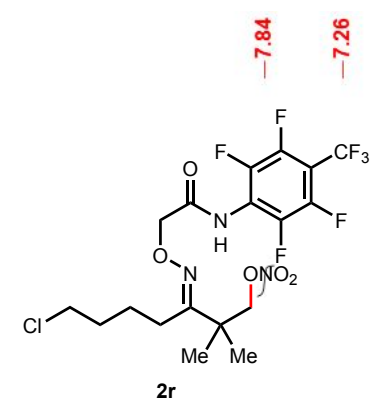

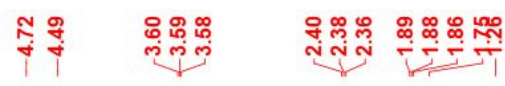

2r
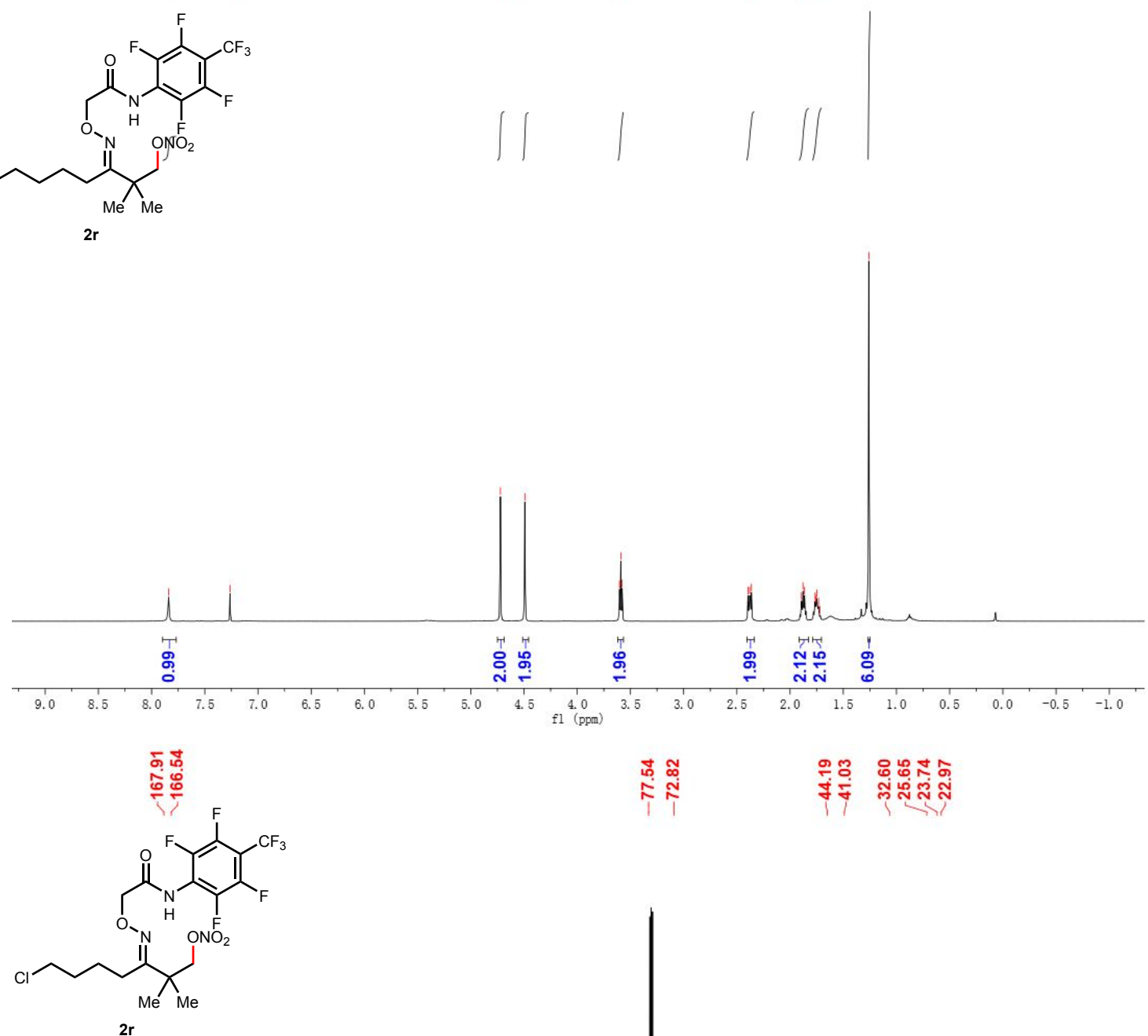

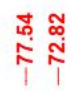

管

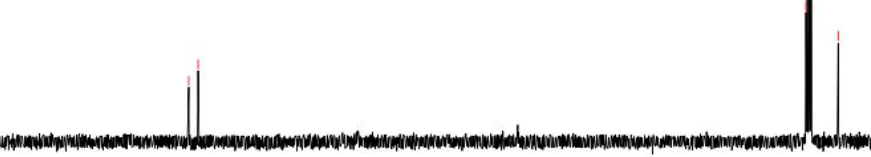

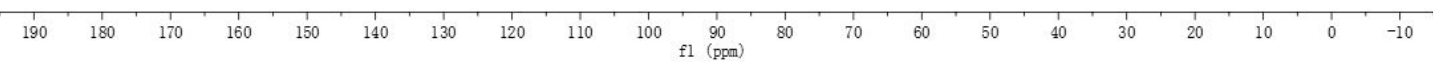



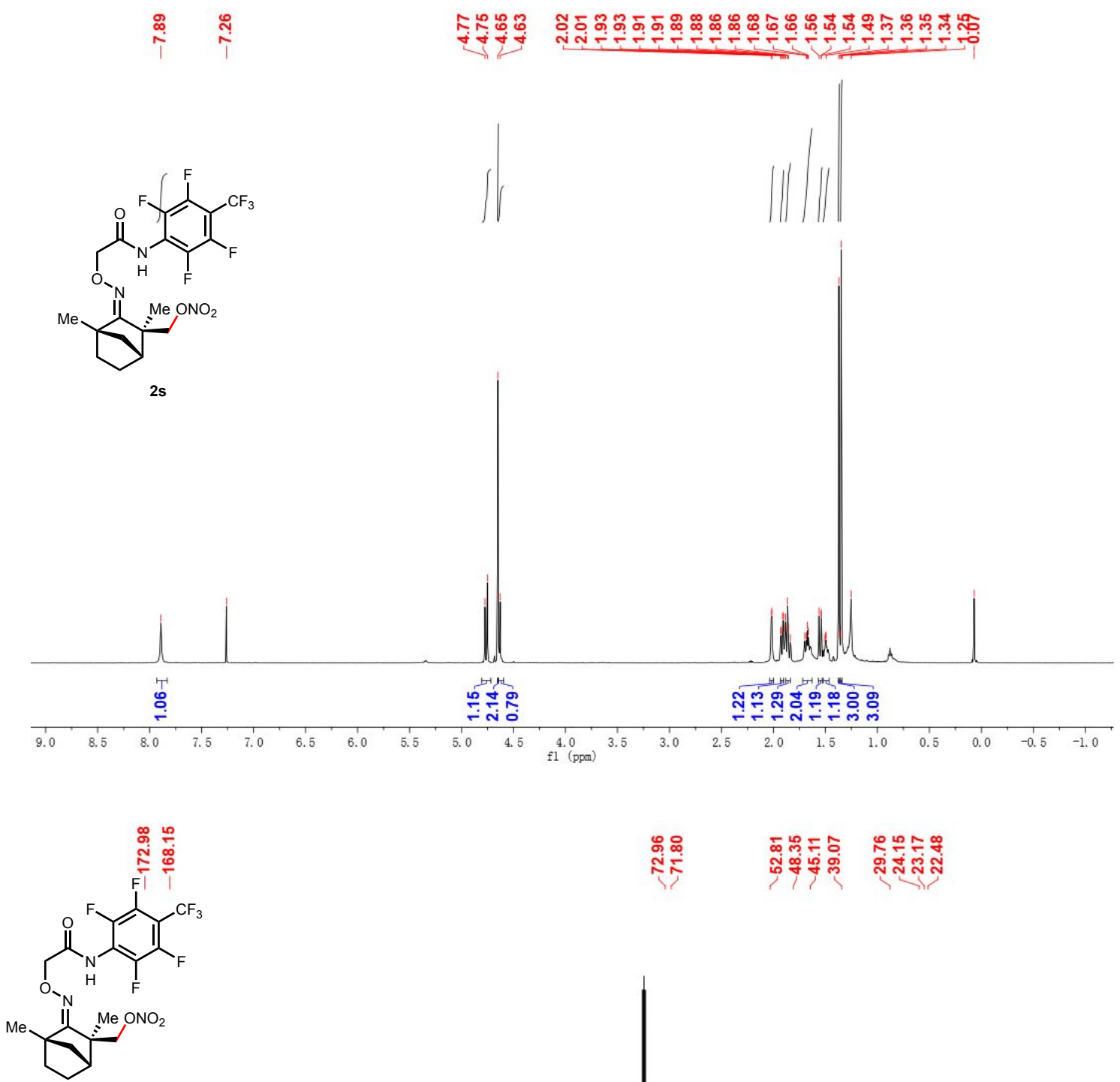

2s

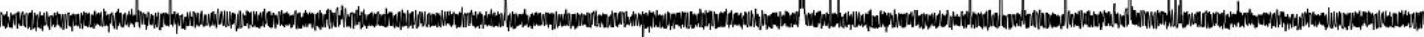

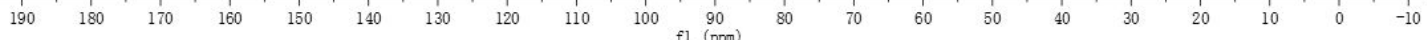




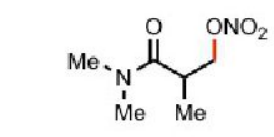

4a

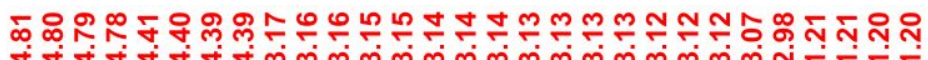

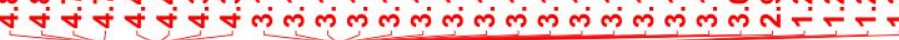
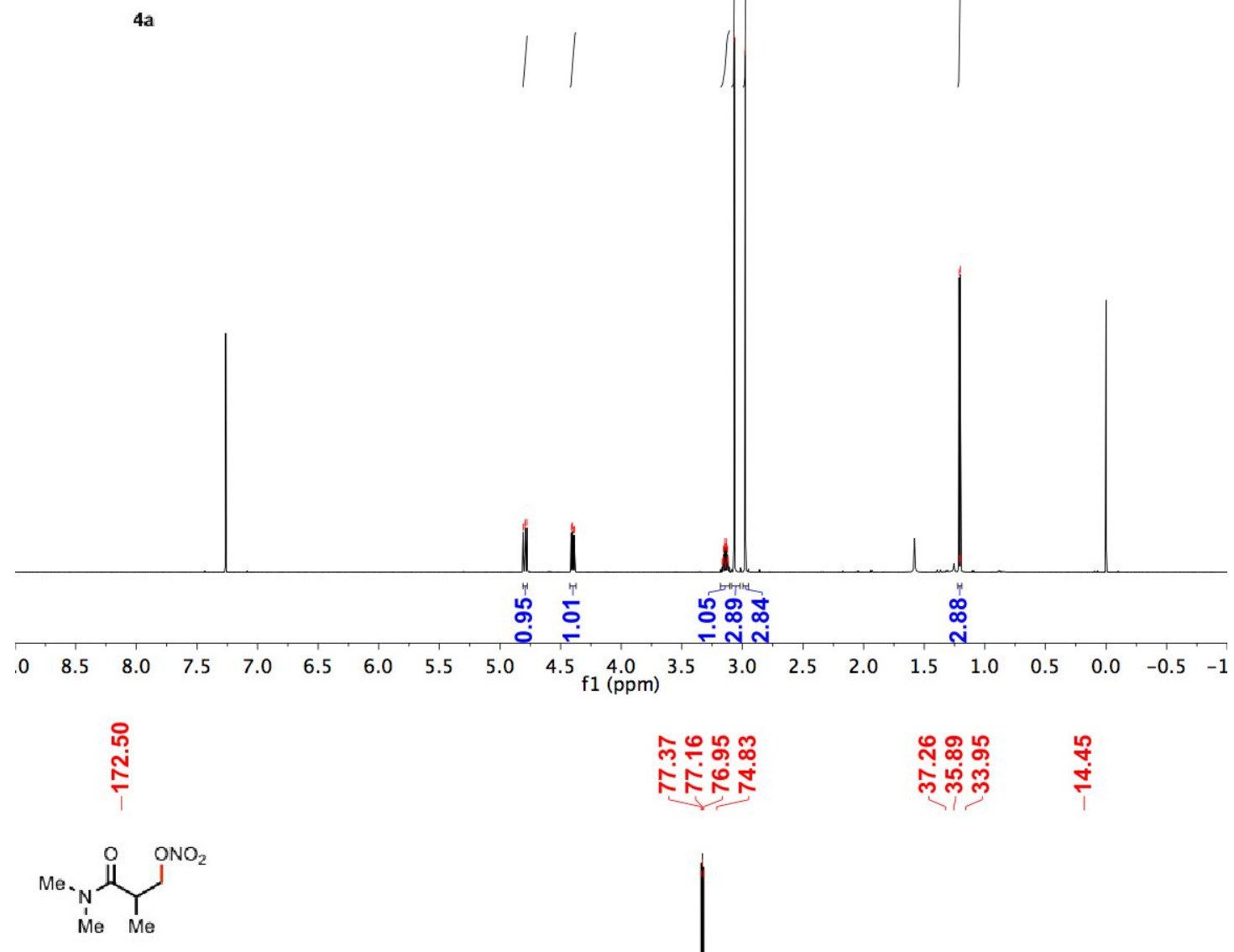

$4 a$

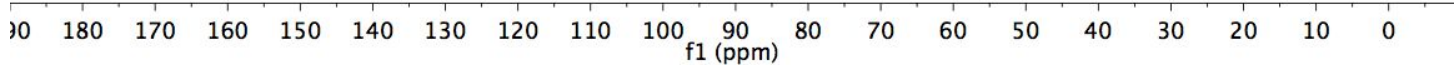




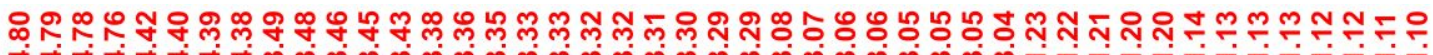
广रें<smiles>CCN(CC)C(=O)C(C)COC</smiles>

$4 b$
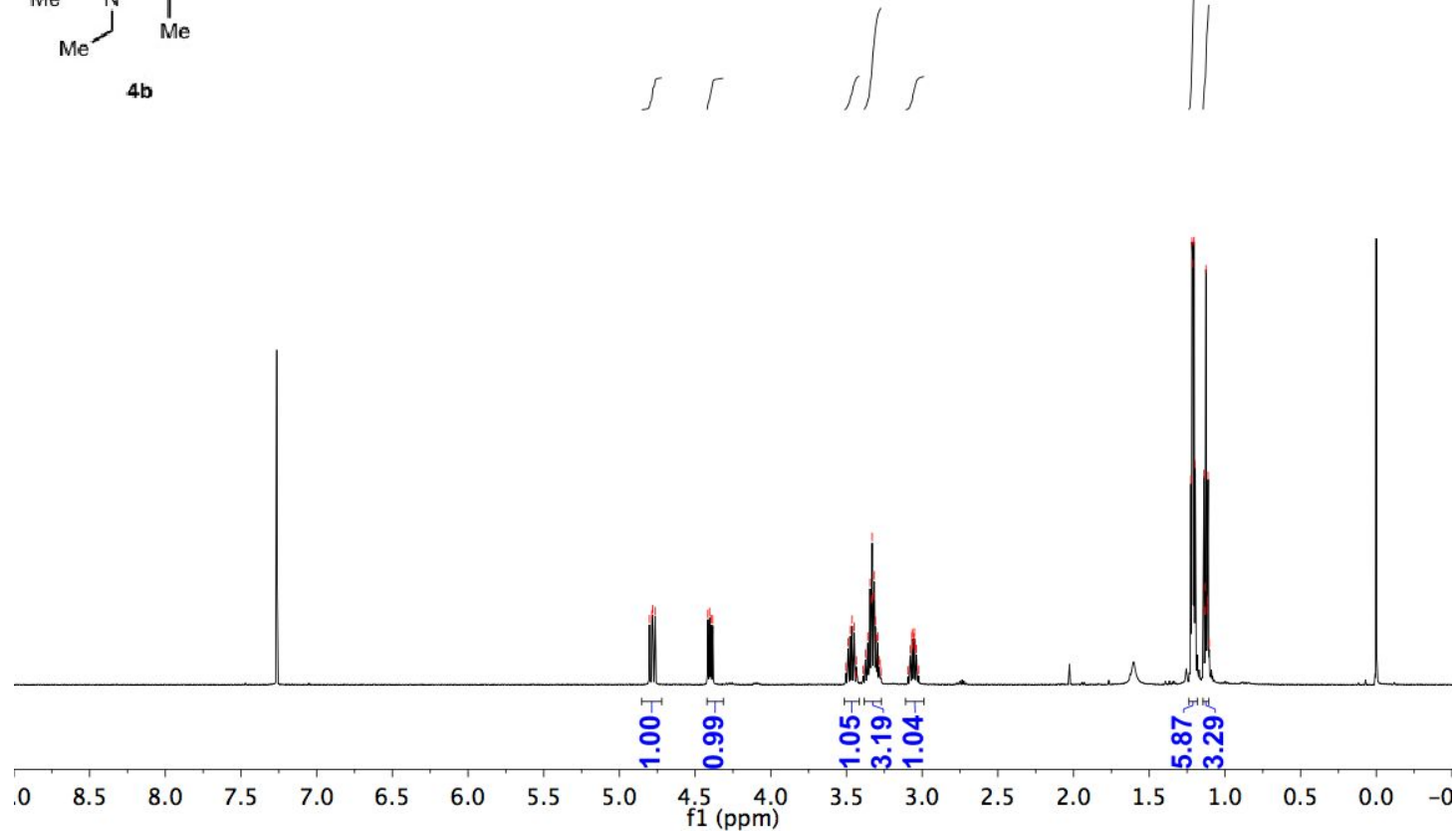

$\stackrel{8}{\stackrel{0}{+\infty}}$

กิำำำ

ANon

울충요 임

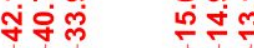<smiles>CCN(CC)C(=O)C(C)COC</smiles>

4b

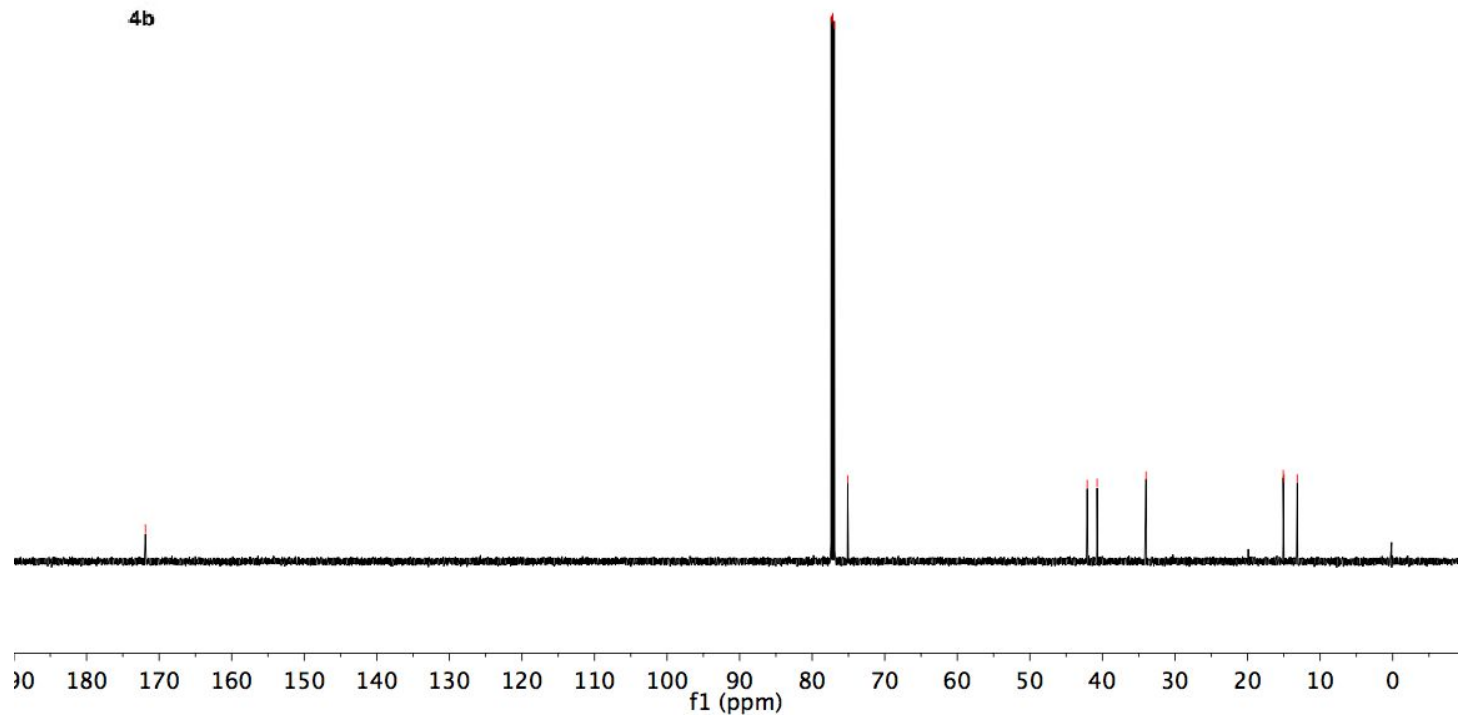




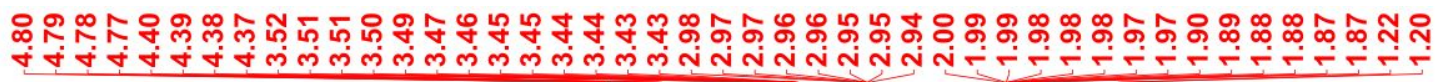<smiles>[NH3+]OCC([NH3+])C(=O)N1CCCC1</smiles>

$4 c$
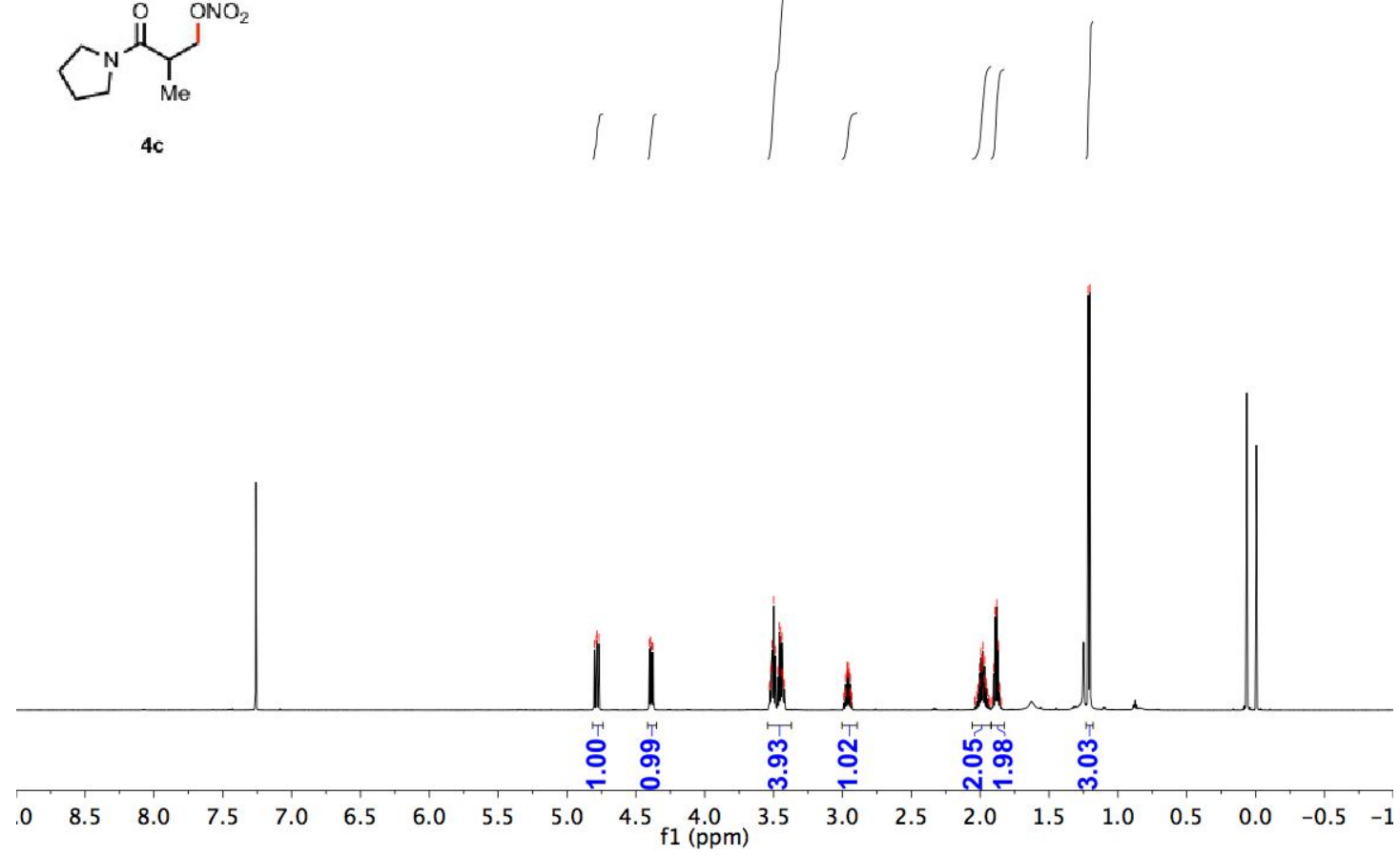

$\stackrel{\infty}{\stackrel{\infty}{\circ}}$

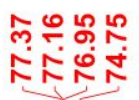

ஜֶׁ

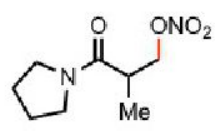

$4 \mathrm{c}$

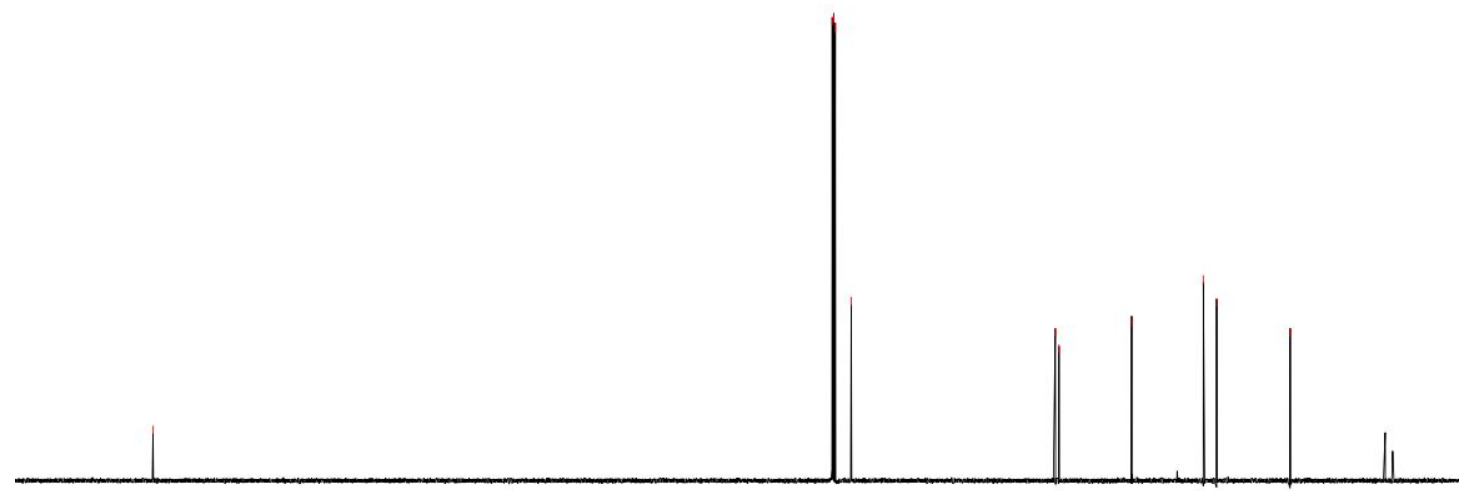

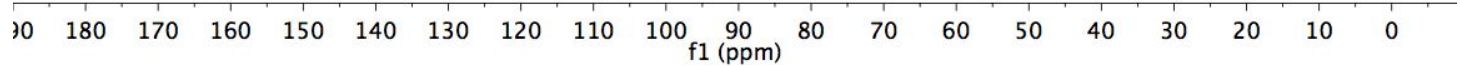




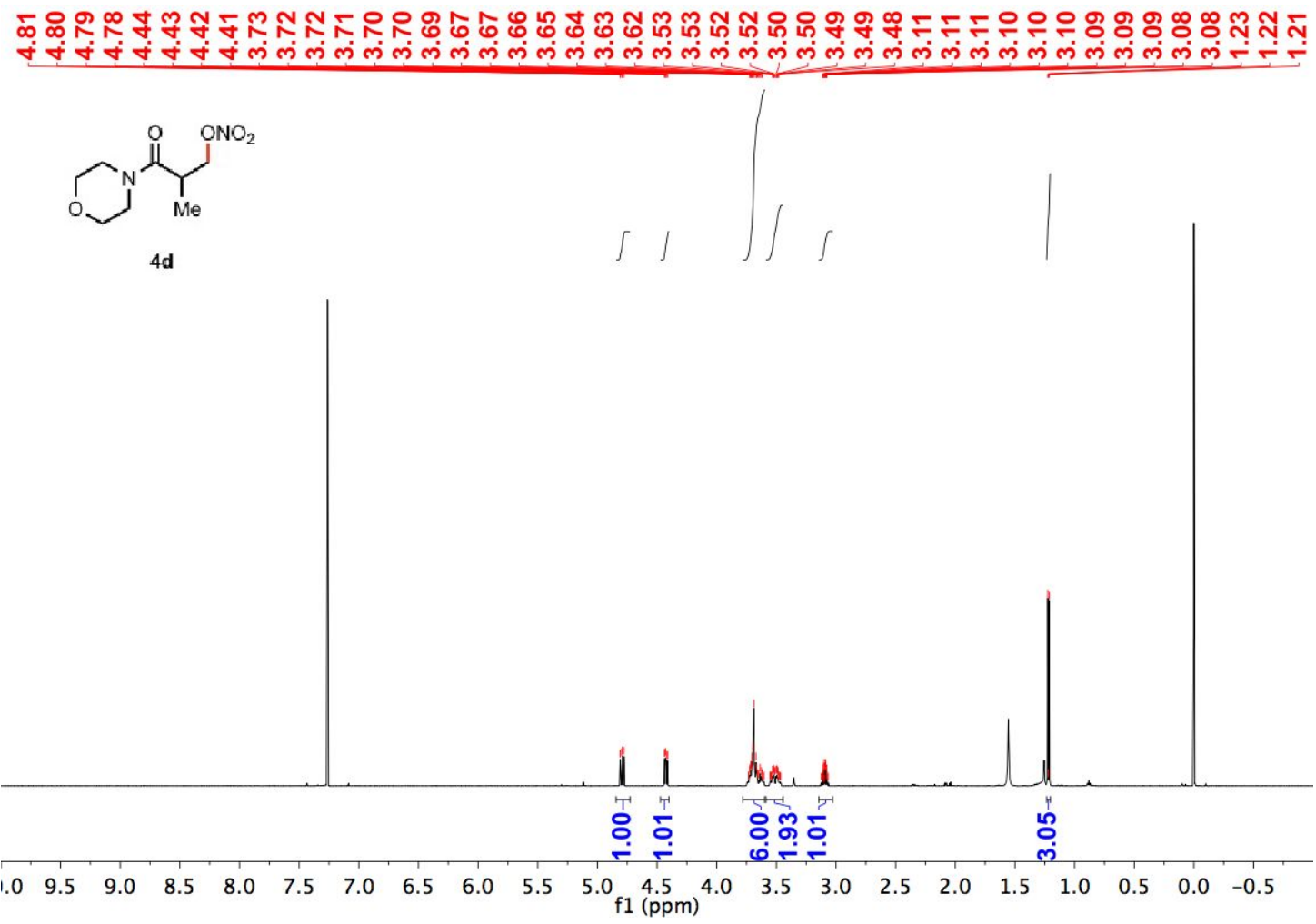<smiles>CC(CO[N+](=O)[O-])C(=O)N1CCOCC1</smiles>

$4 d$

ᄁึษั丶

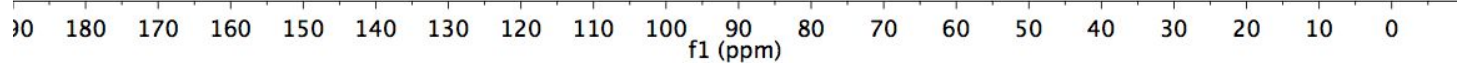



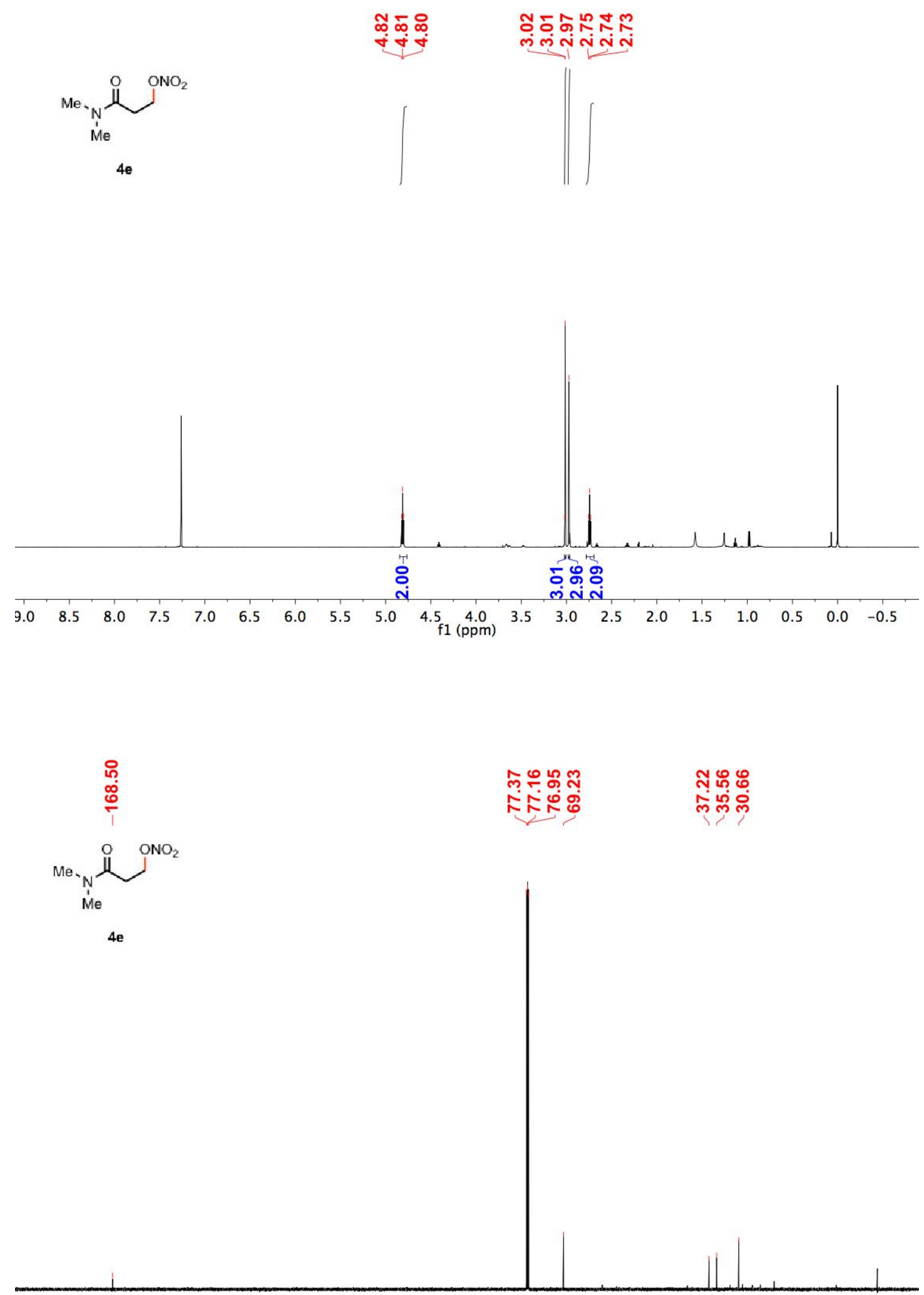

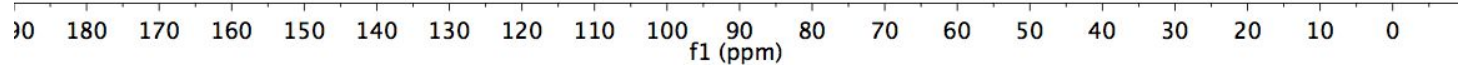



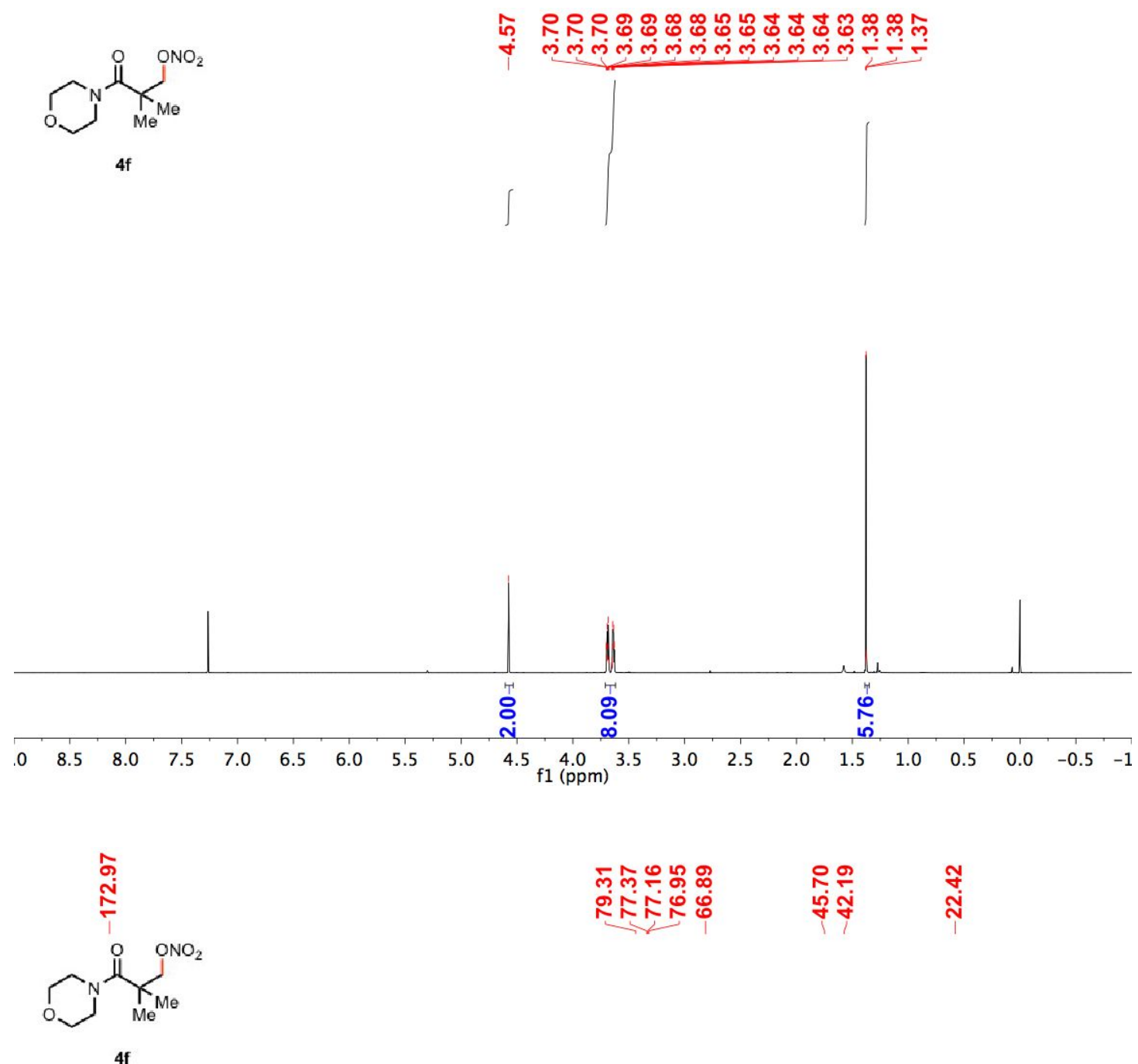

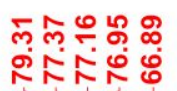

월

$\stackrel{\text { มิ }}{\text { บ }}$

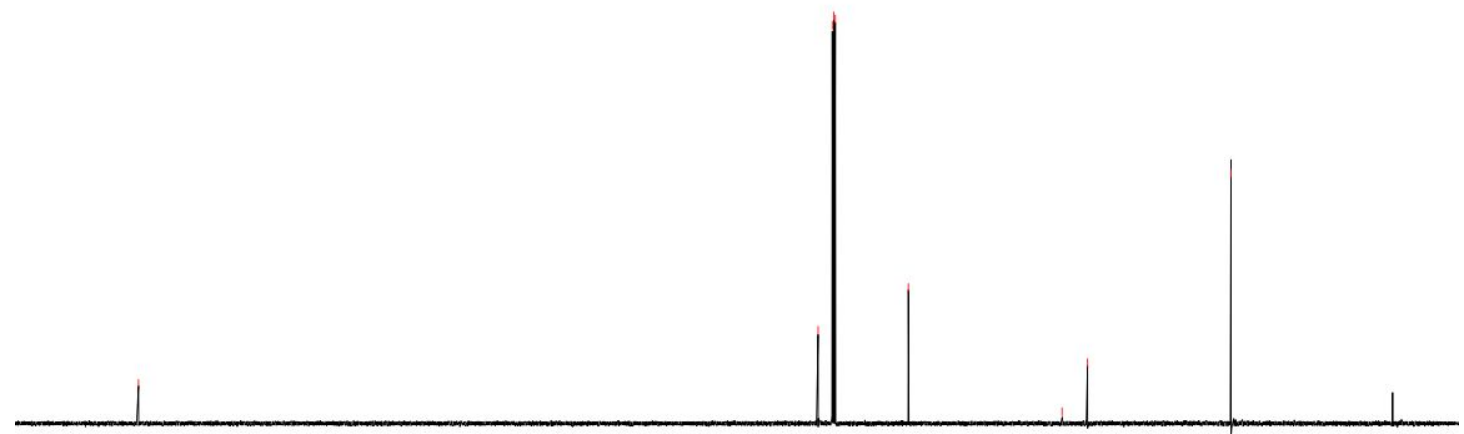

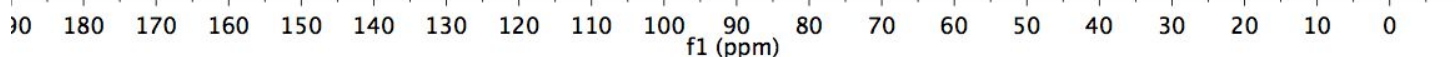




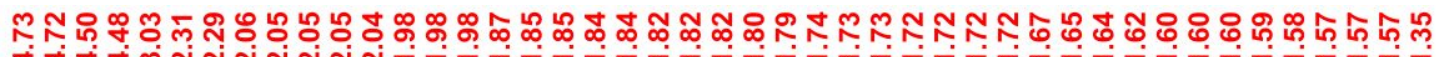

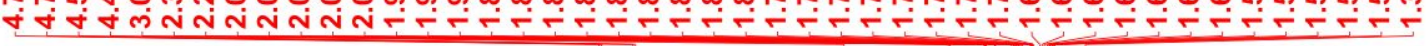<smiles>COC(=O)C(O)(CC1CC1)C(=O)N(C)C</smiles>

$4 \mathrm{~g}$
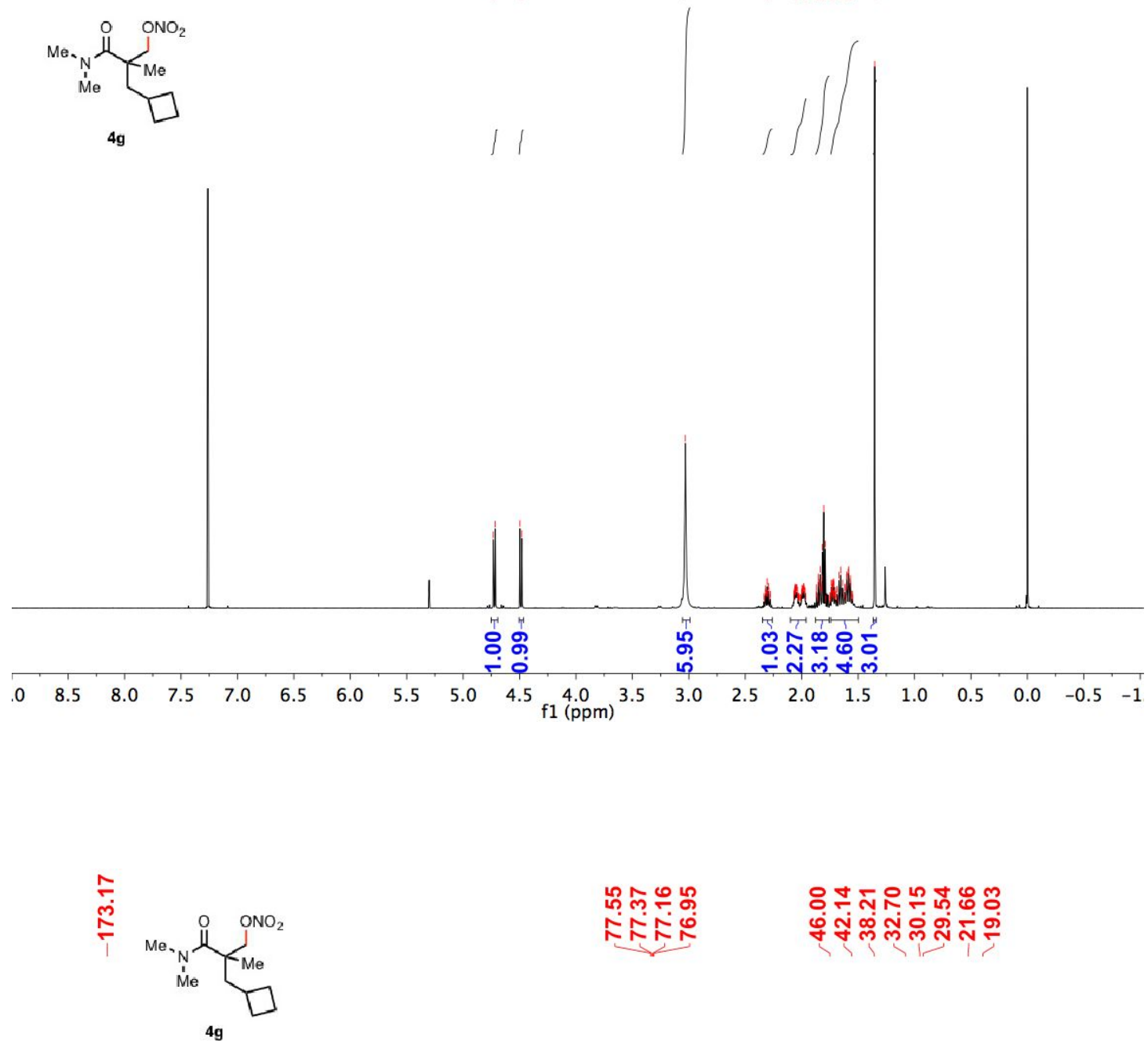

นึกิ

Nín

ठㄴำㄴำ뉴유

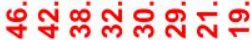

$4 \mathrm{~g}$

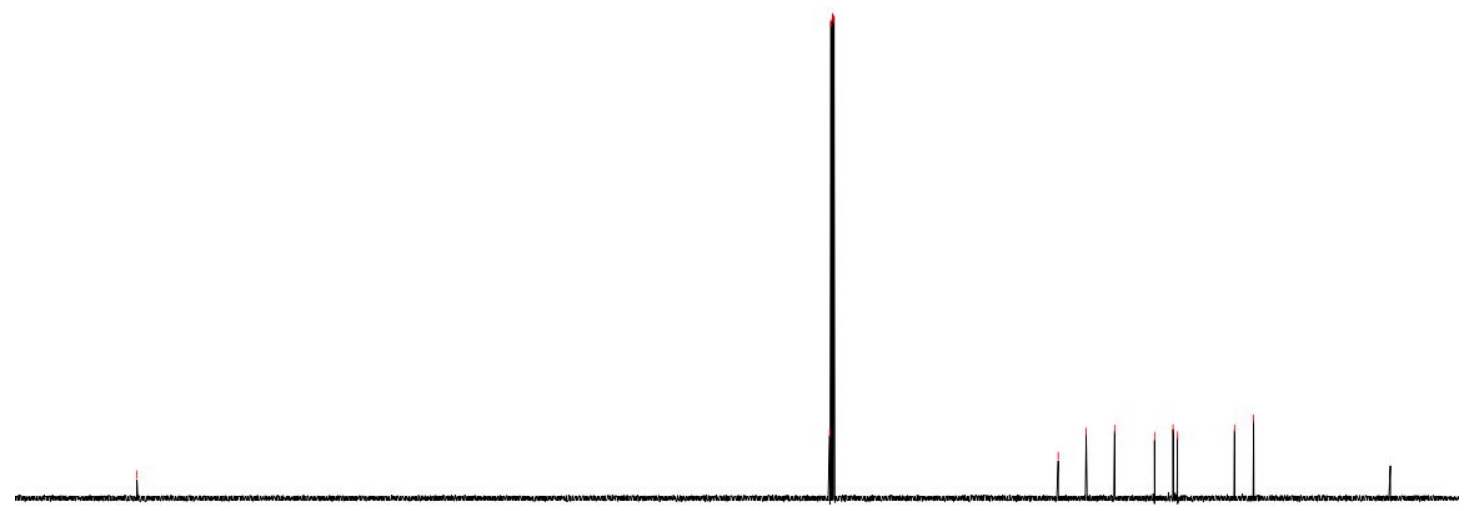

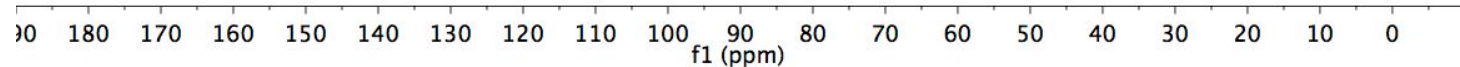




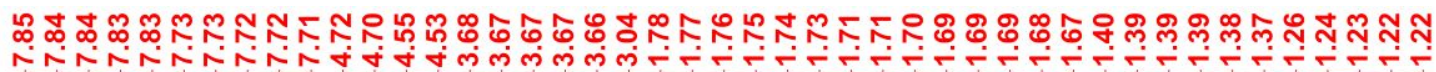<smiles>CN(C)OCC(CCCCN1C(=O)c2ccccc2C1=O)(CO[N+]([O-])([O-])[O-])C(=O)N(C)C</smiles>

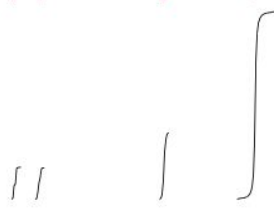

$4 \mathrm{~h}$
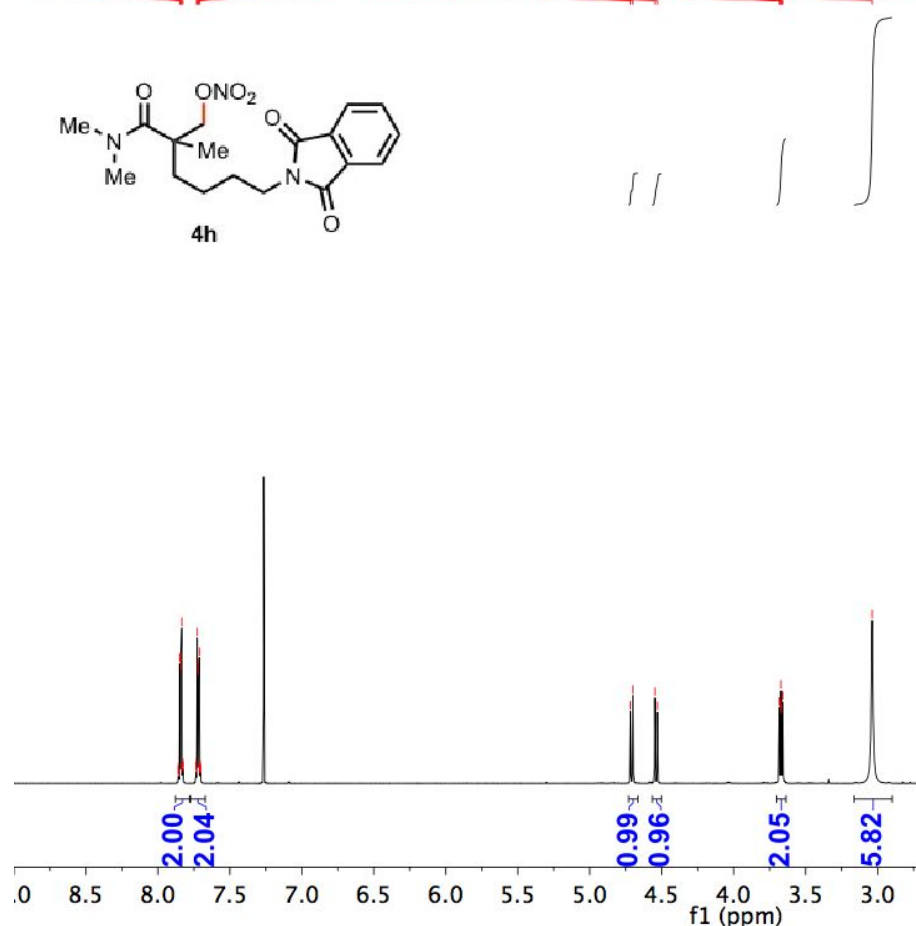

\section{क्षे}

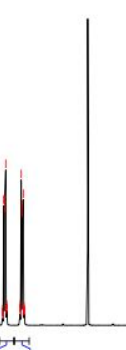

7.5

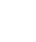




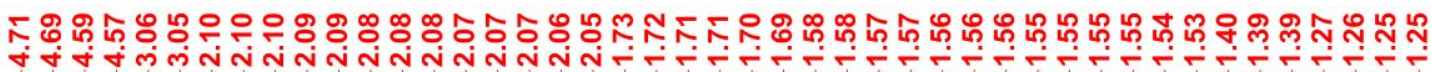<smiles>CCCCCC(CO[N+](=O)[O-])(CO[N+](=O)[O-])C(=O)N(C)[Na]</smiles>

$4 \mathrm{i}$
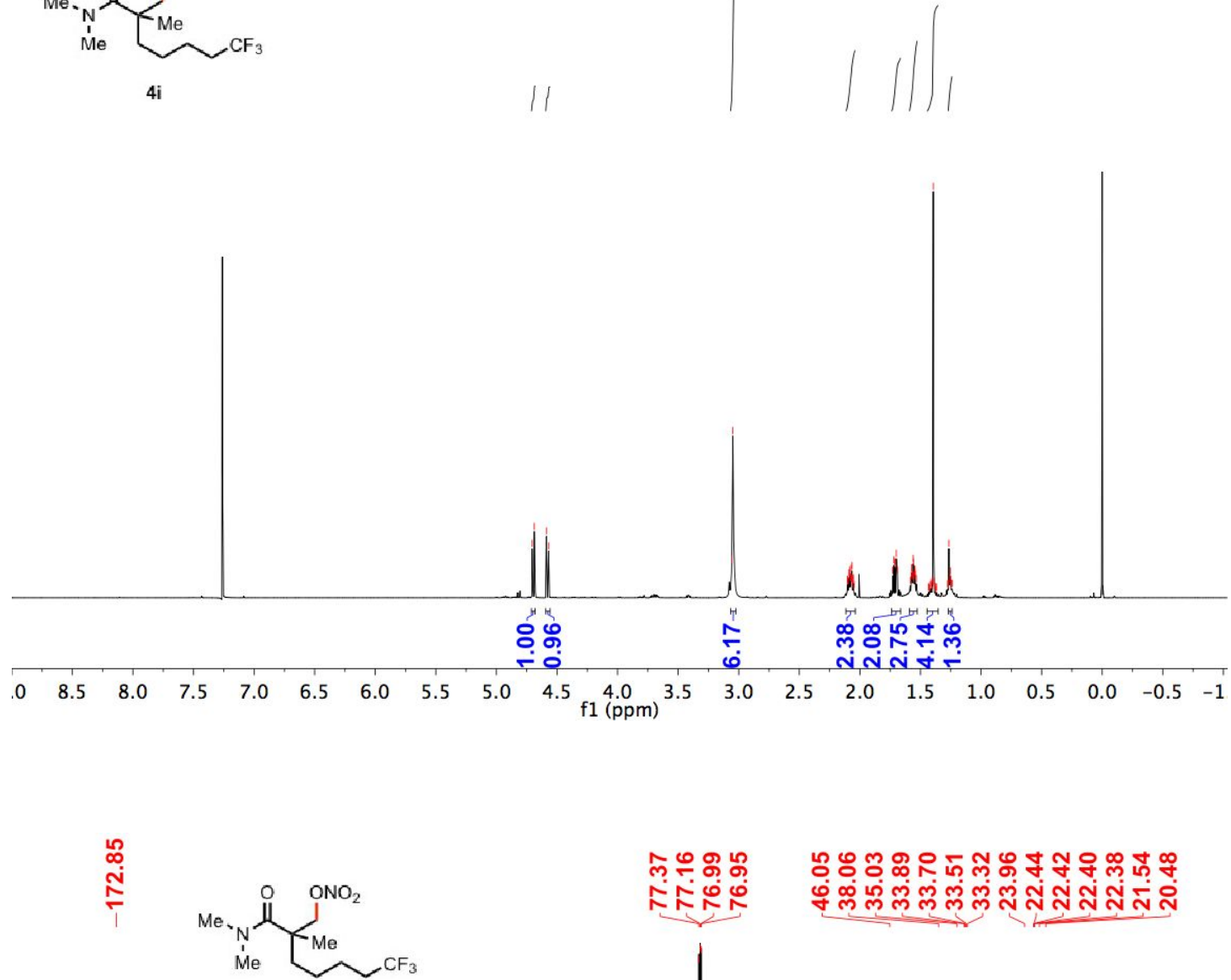

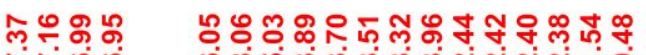

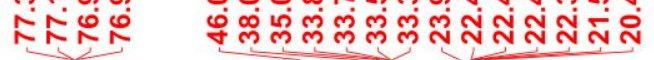

$4 \mathrm{i}$

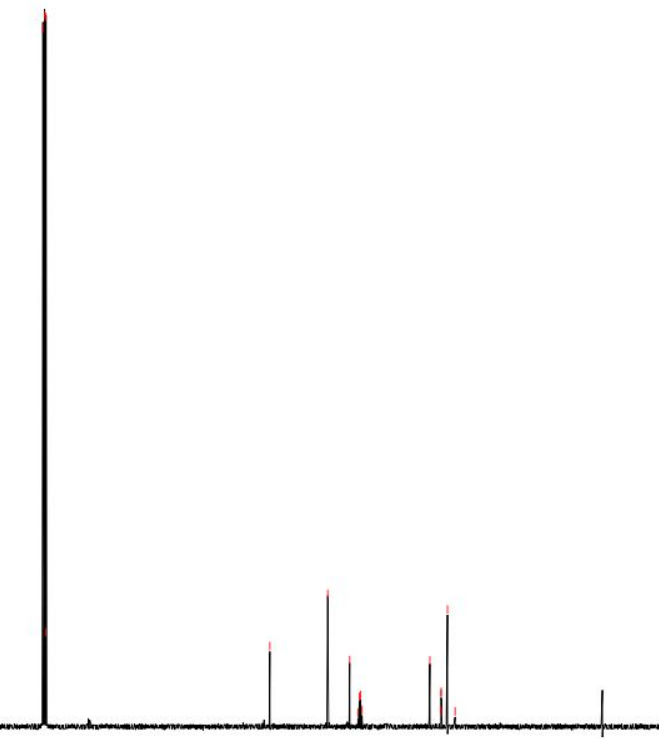

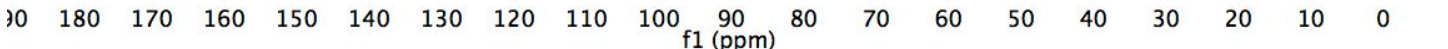




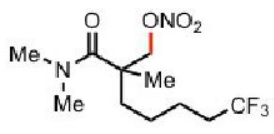

$4 i$

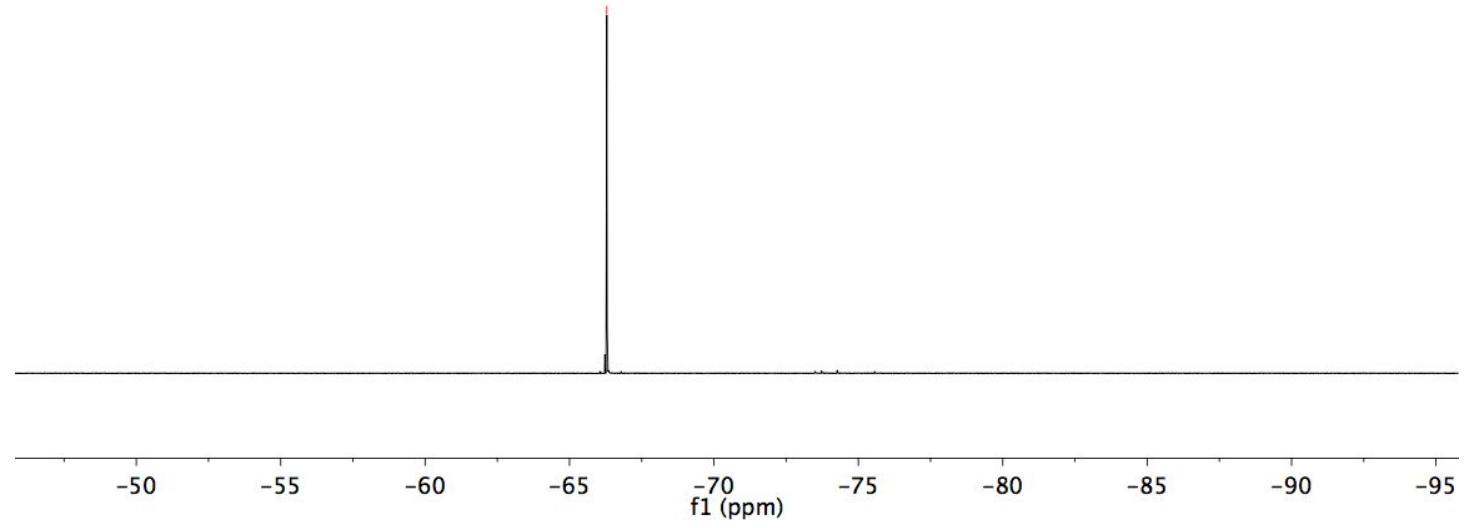



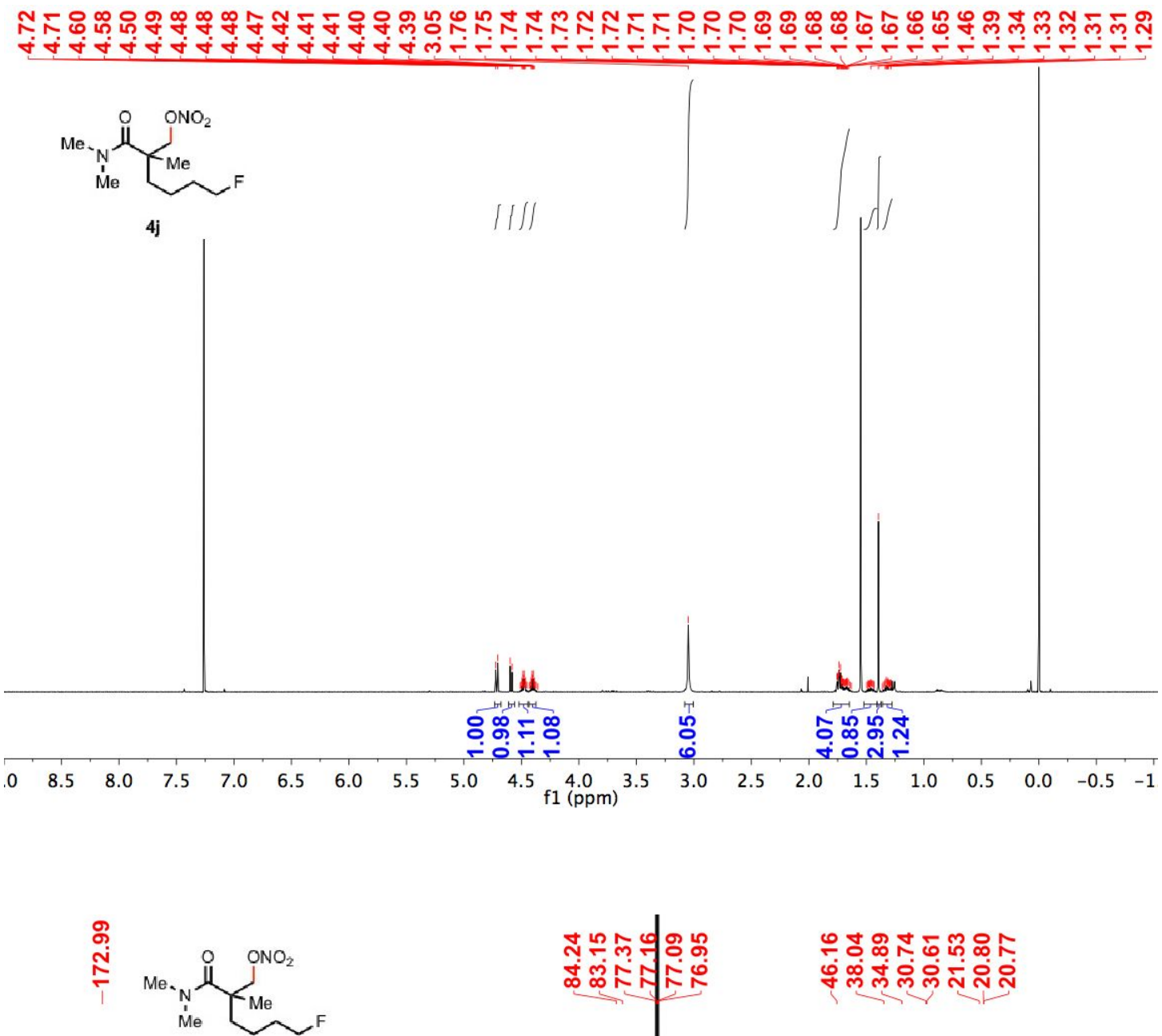

$4 \mathbf{j}$

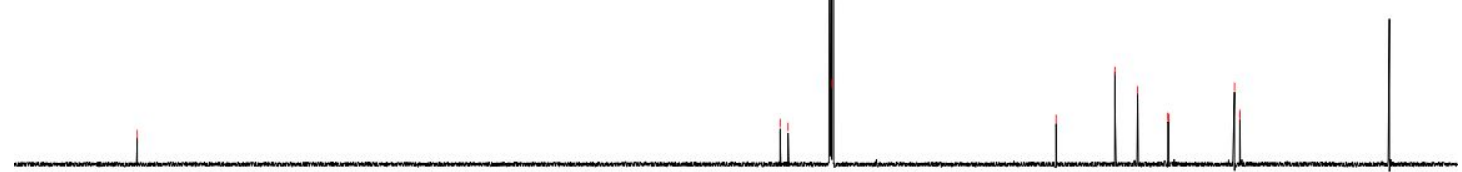

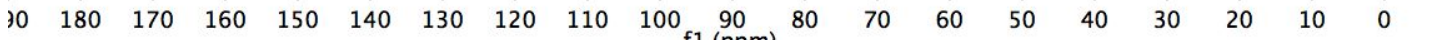



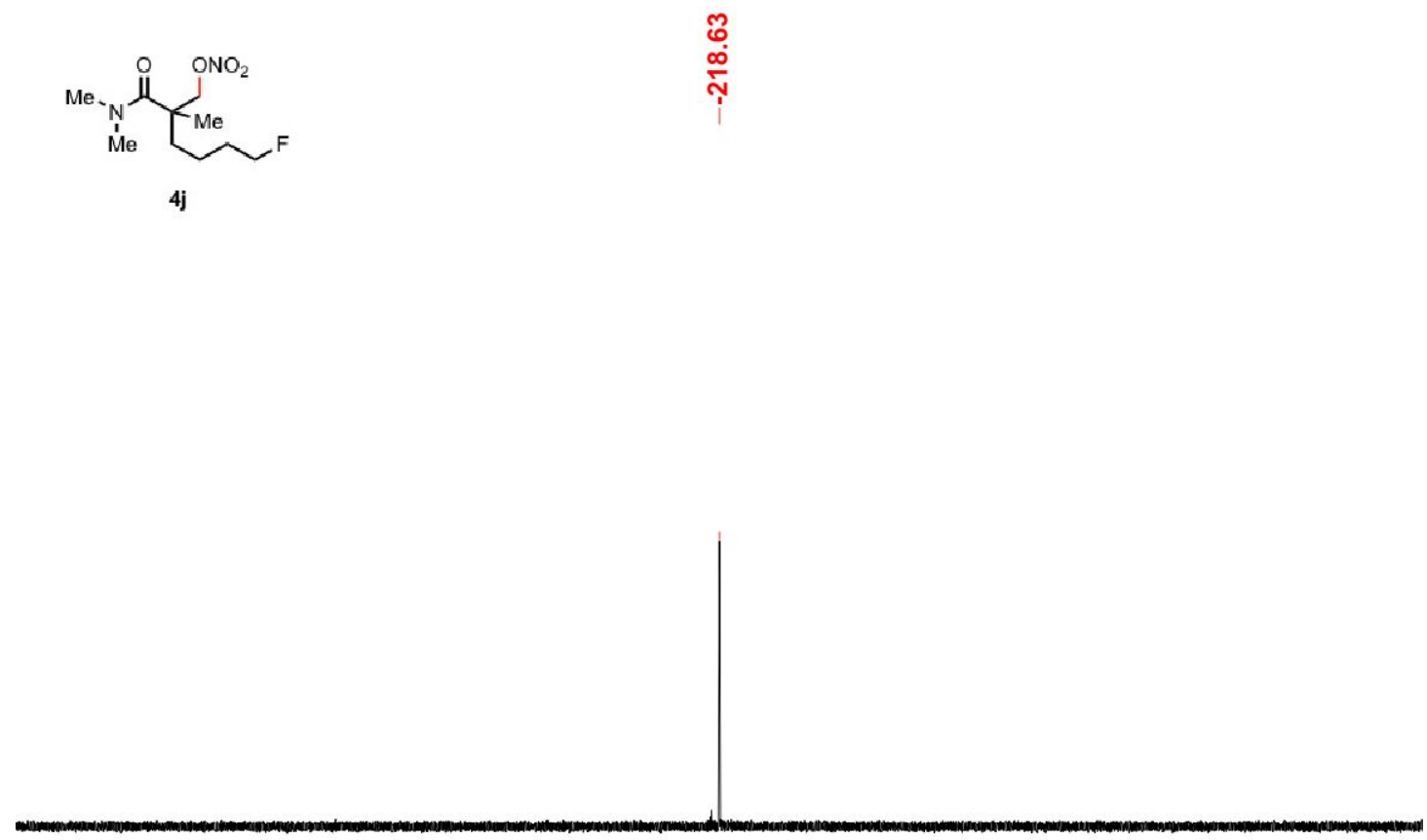


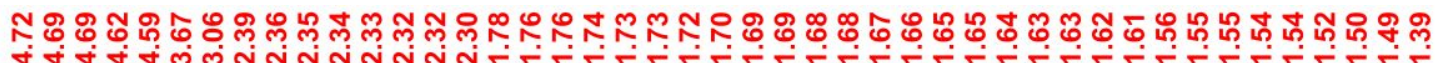

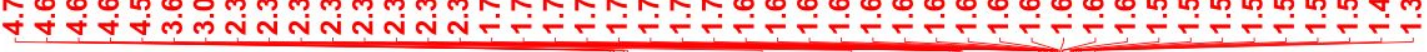<smiles>CC(=O)CCCC(CO[N+](=O)[O-])(CO[N+](=O)[O-])C(=O)N(C)[Na]</smiles>

$4 k$
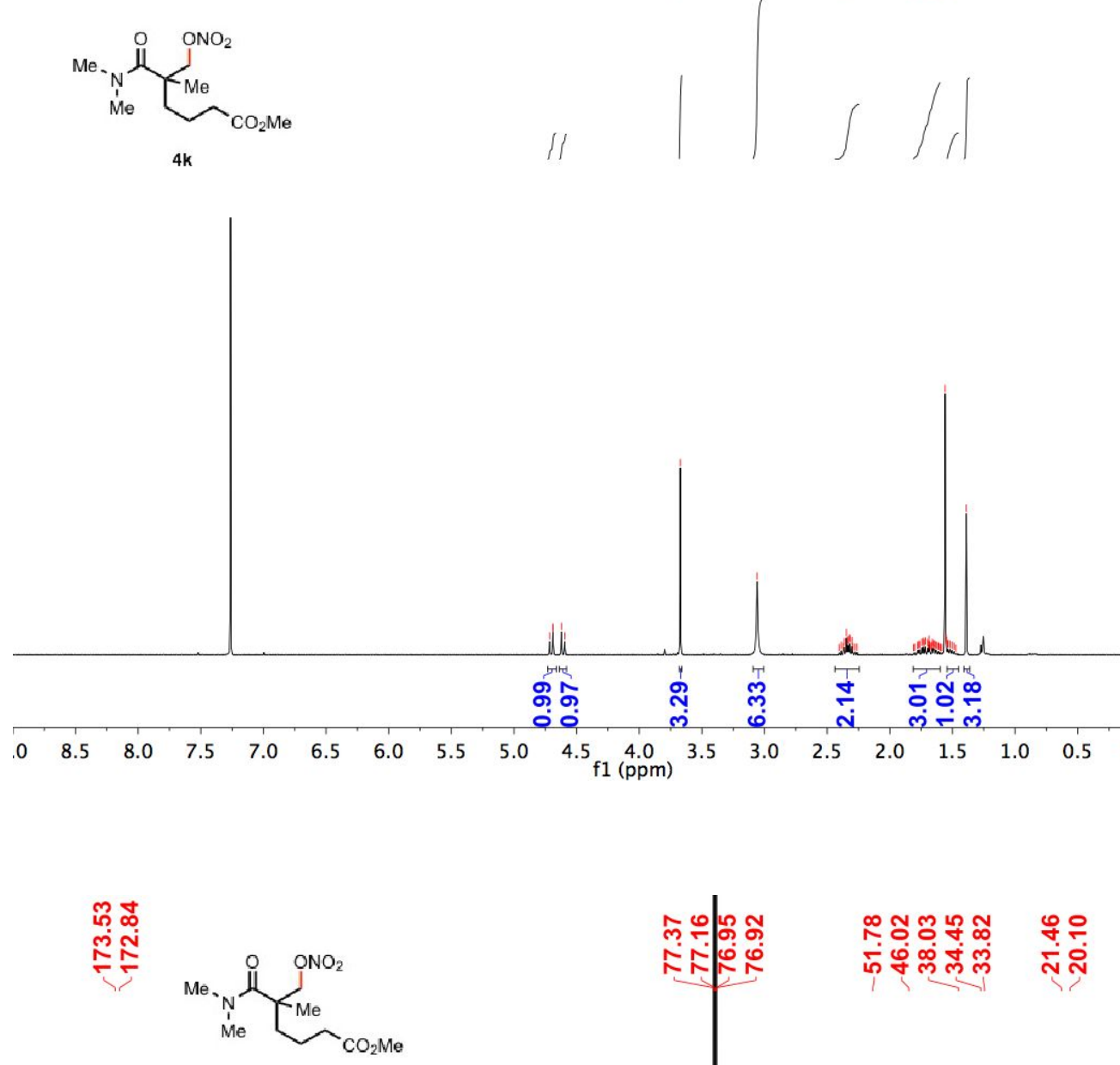

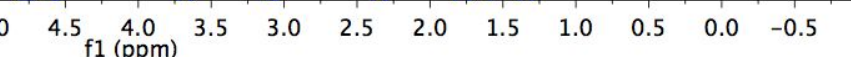

$4 k$

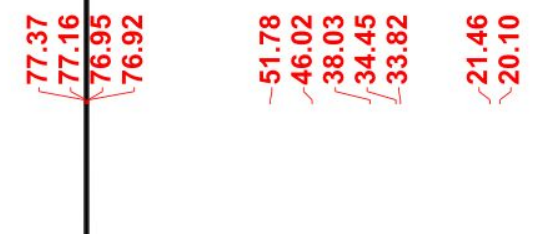

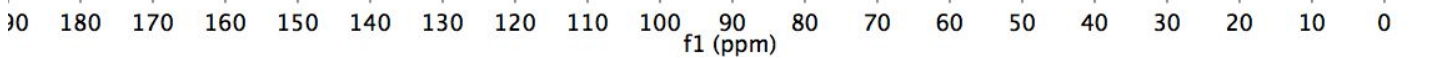




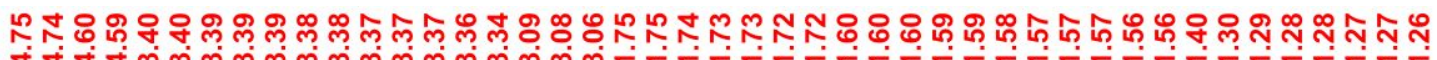

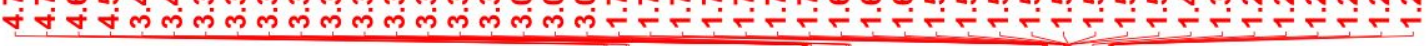
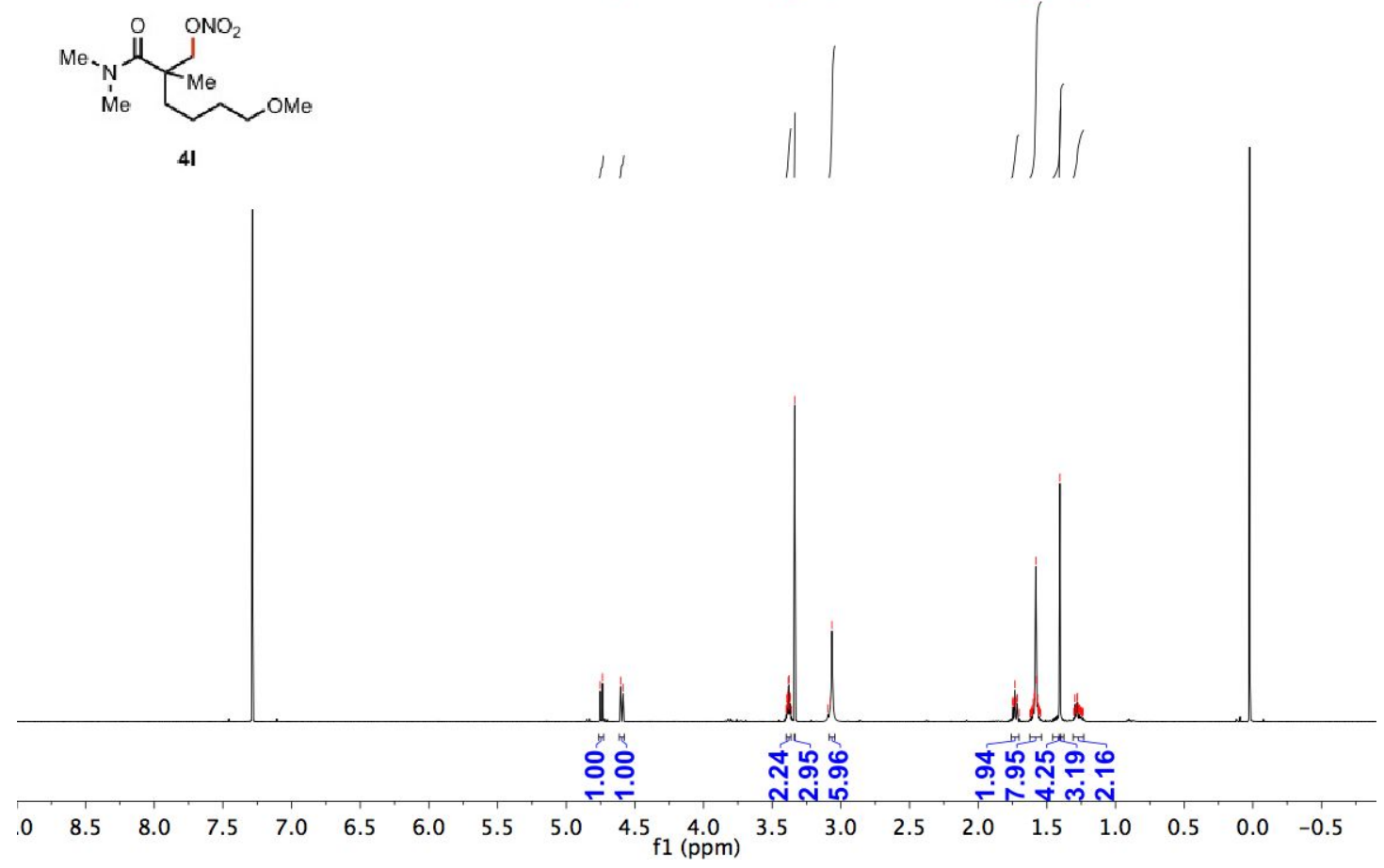

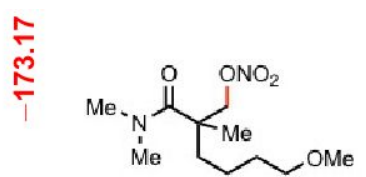

41

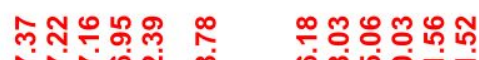

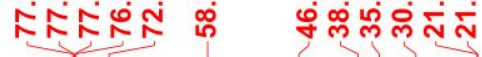

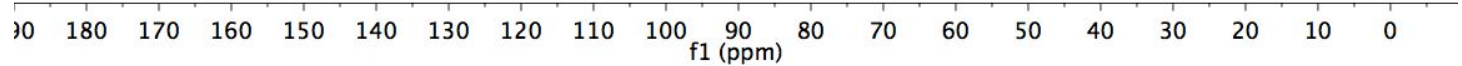




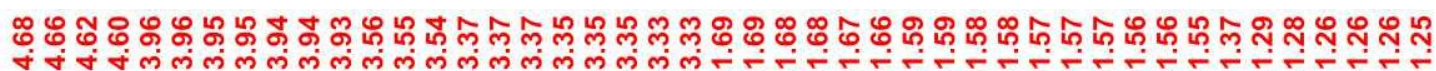<smiles>COC(O)C(=O)C1CCC2CCOCC2C1</smiles>

$4 \mathrm{~m}$
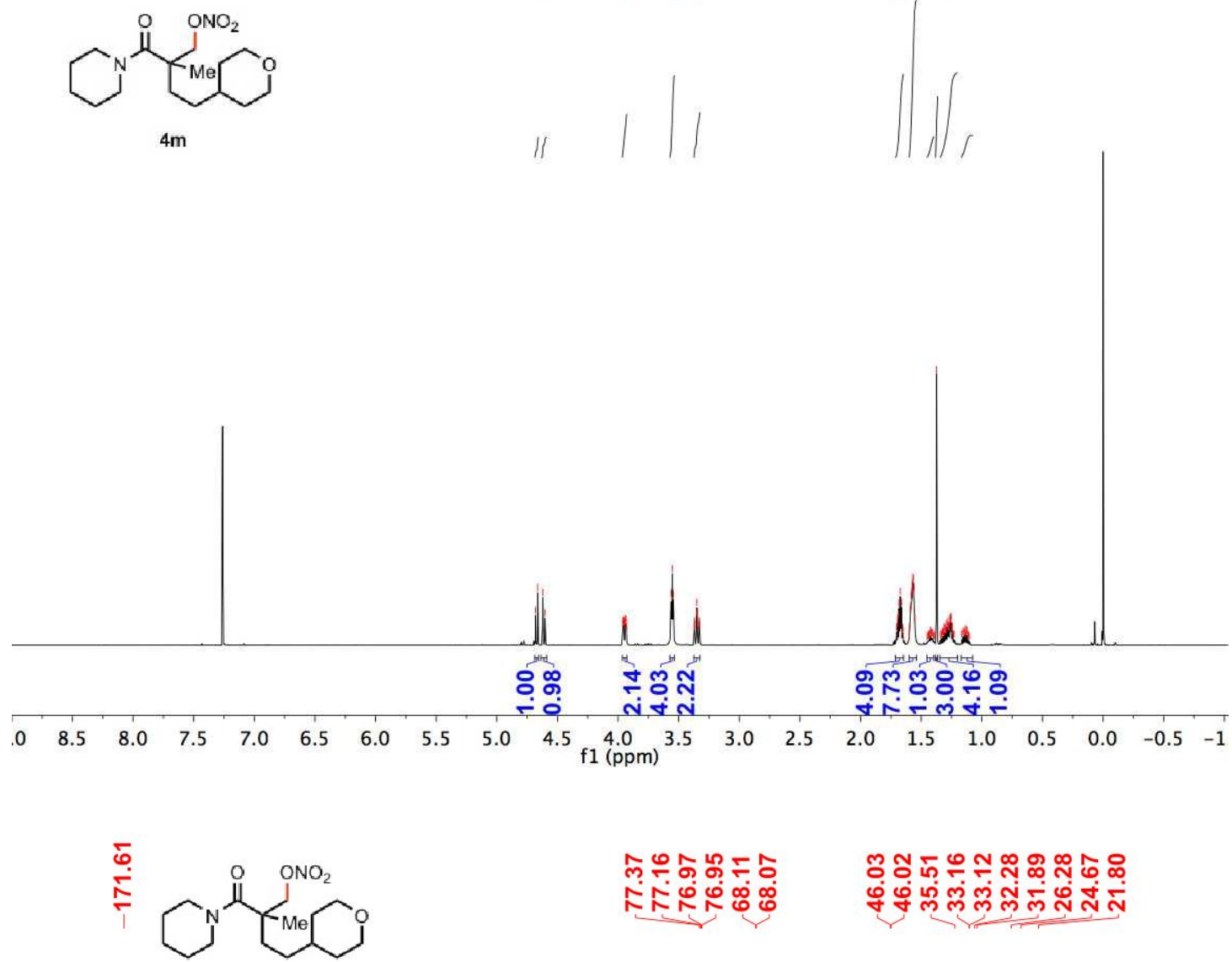

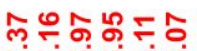

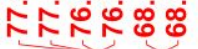

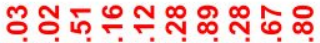

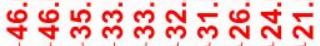

$4 m$

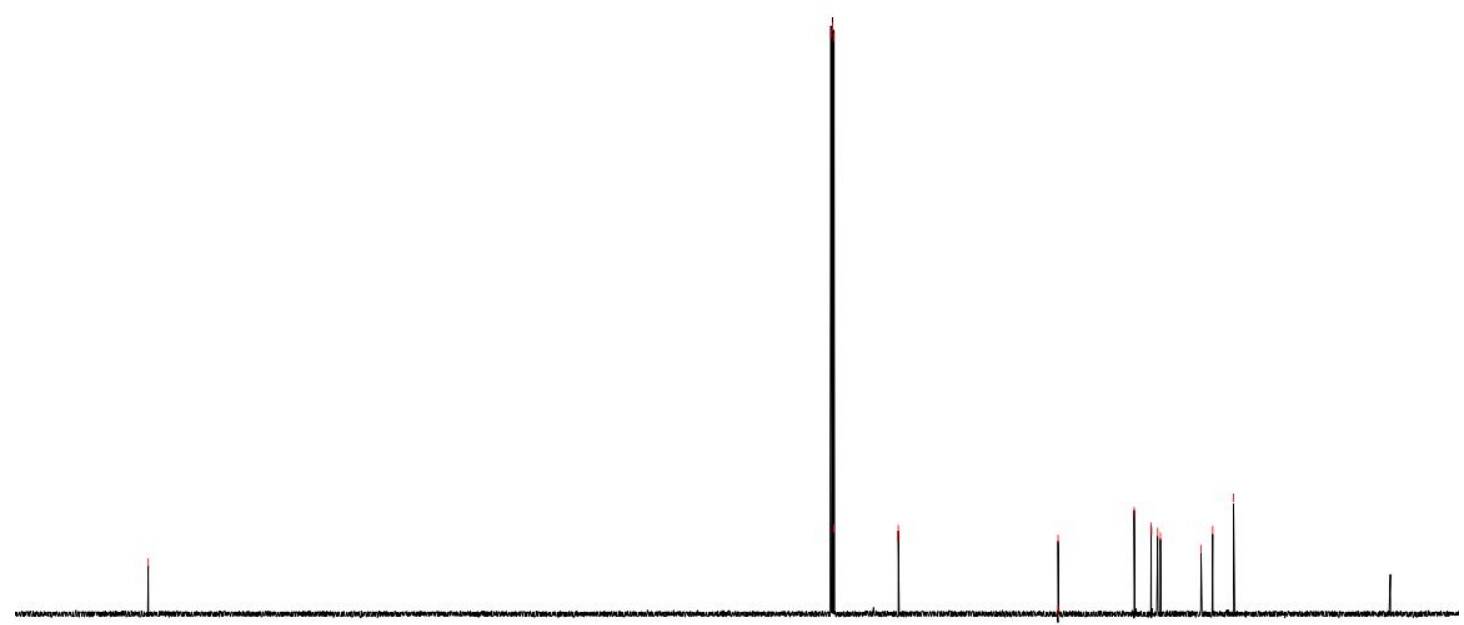

$\begin{array}{llllllllllllllllllll}30 & 180 & 170 & 160 & 150 & 140 & 130 & 120 & 110 & 100 & \begin{array}{c}90 \\ \mathrm{fpm})\end{array} & 80 & 70 & 60 & 50 & 40 & 30 & 20 & 10 & 0\end{array}$ 


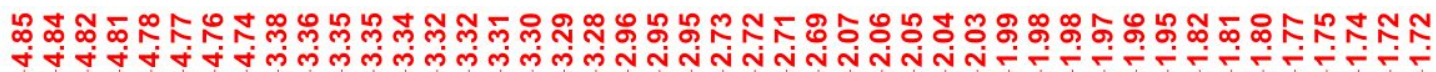
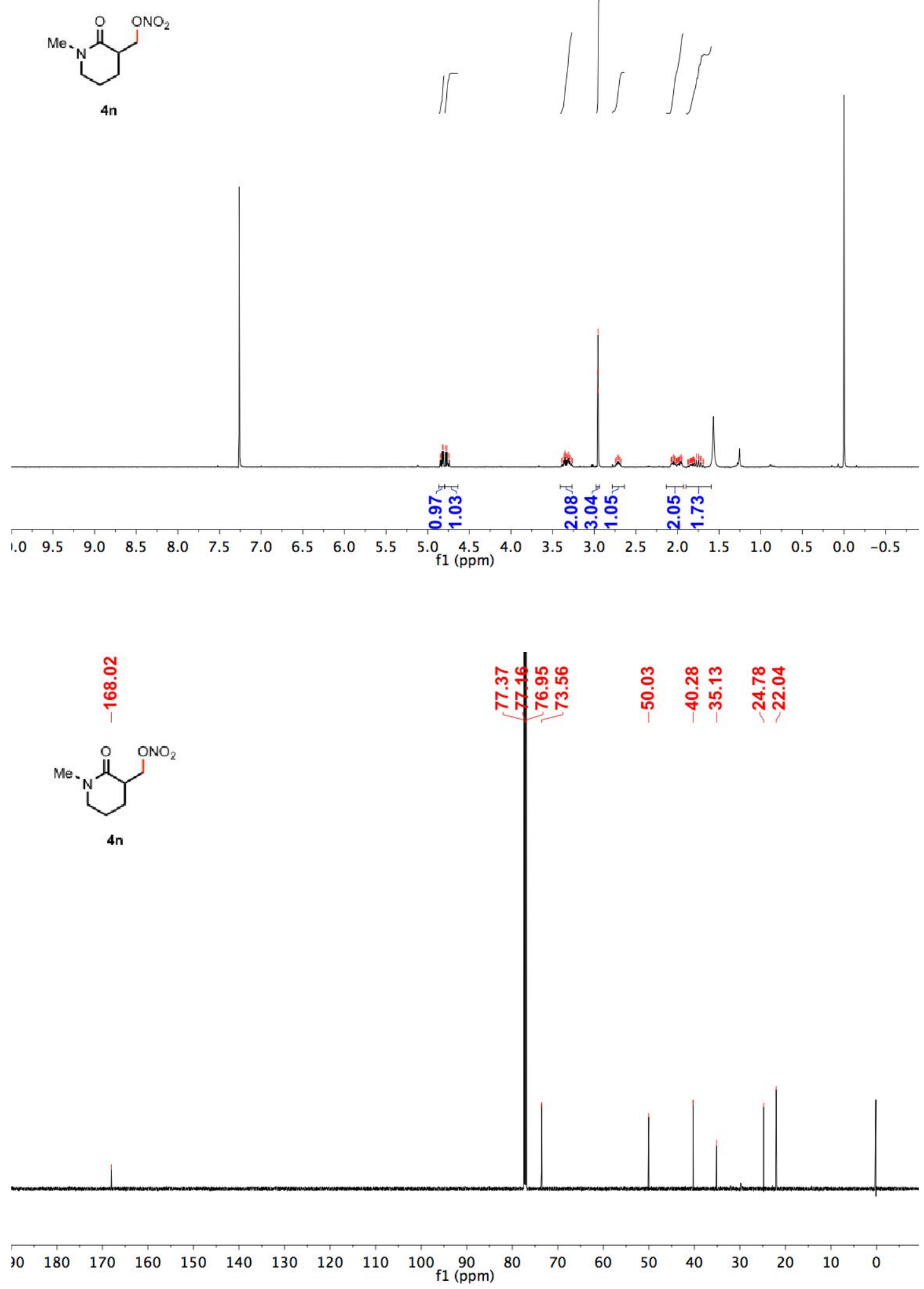


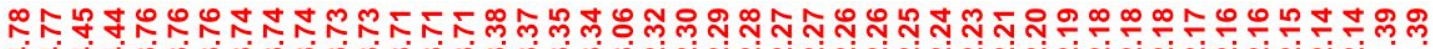

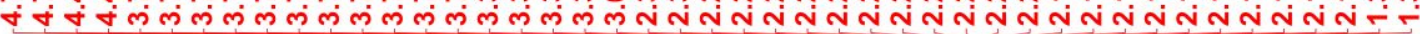

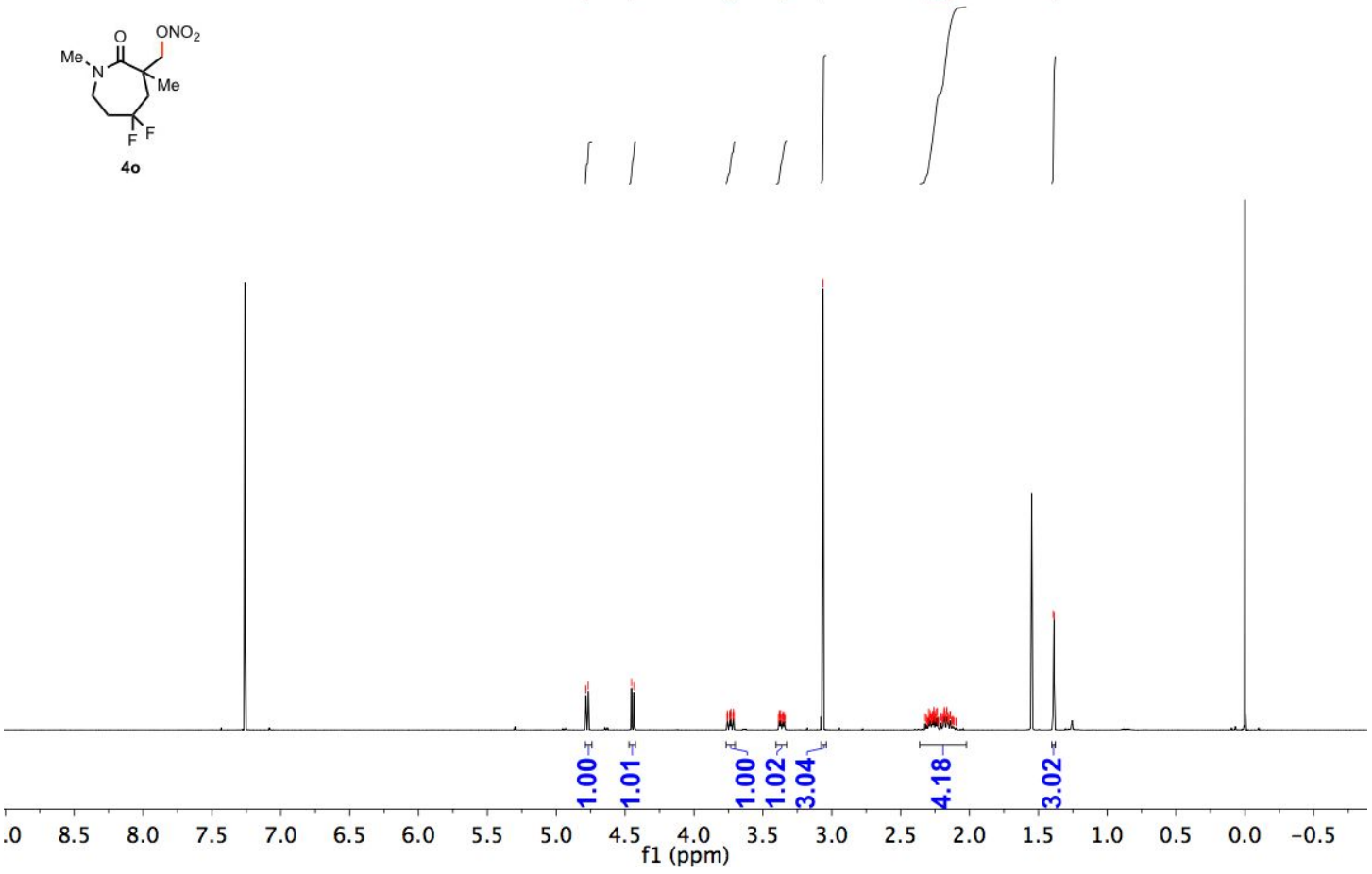

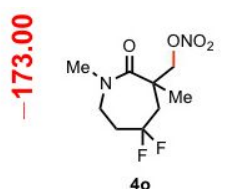

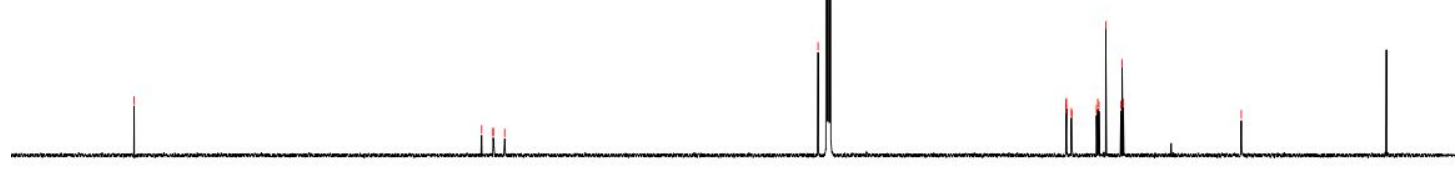

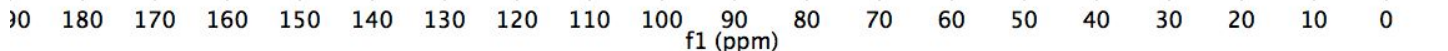


นำ ำก

$\underbrace{M{ }_{F}^{N}}_{F F} \underbrace{\mathrm{ONO}_{2}}_{M e}$

œ市吊

40

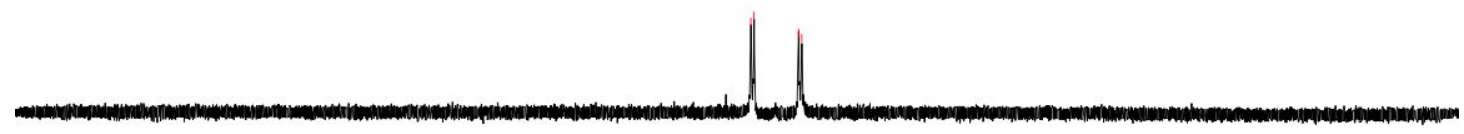

$\begin{array}{lllllllllllllllllllll} & 50 & 30 & 10 & -10 & -30 & -50 & -70 & -90 & -110 & -130 & -150 & -170 & -190 & -210 & -23\end{array}$ 


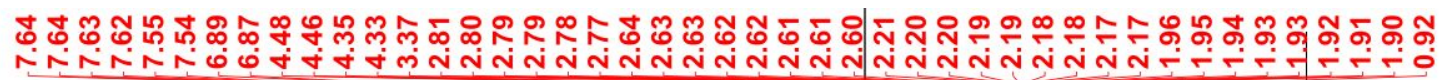

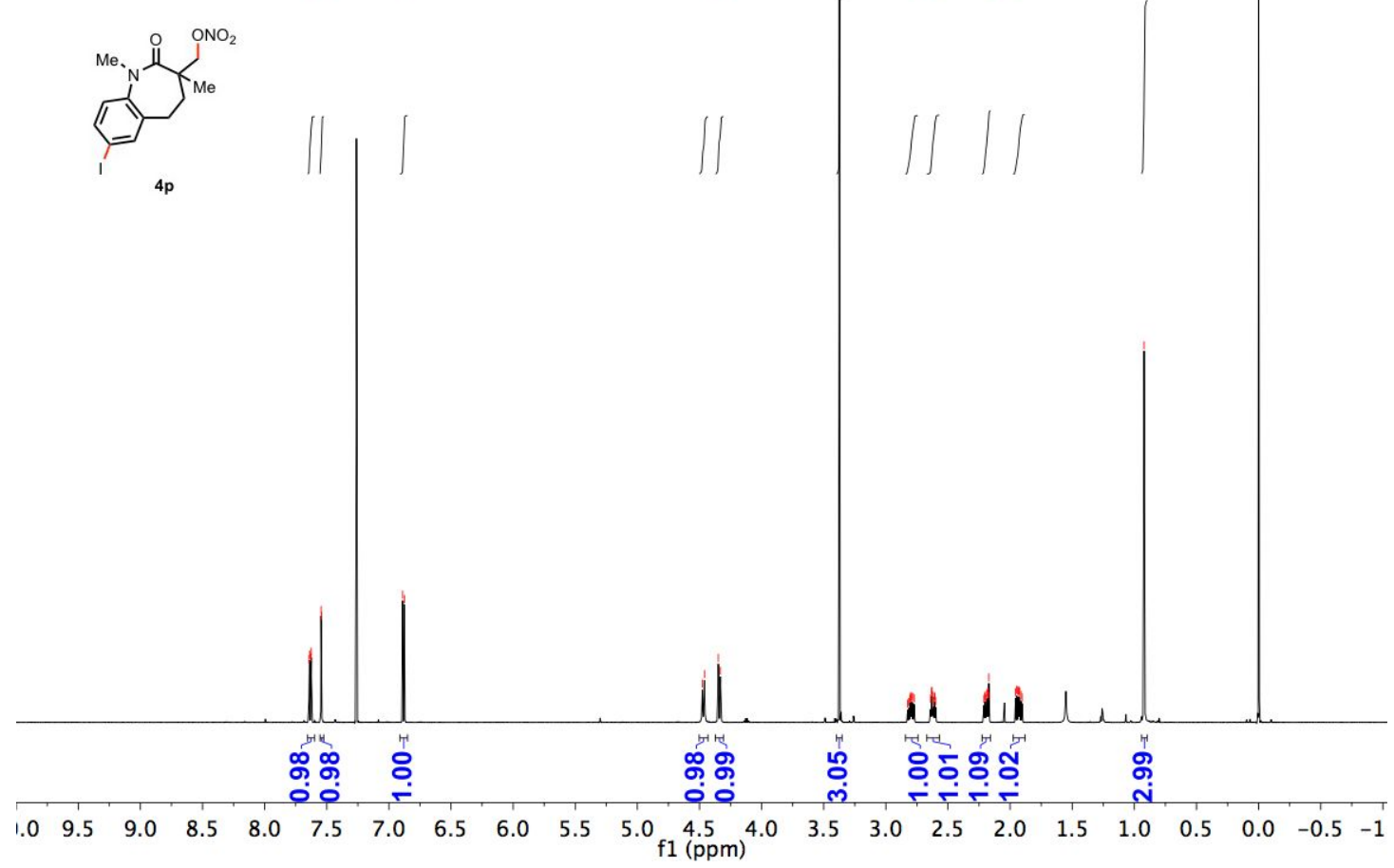

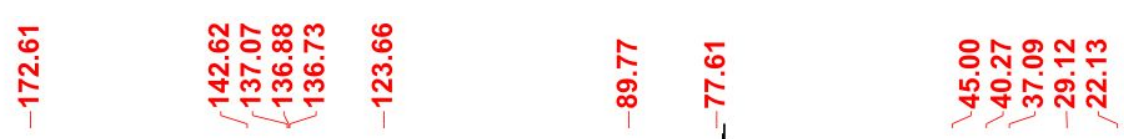

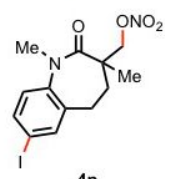

$4 \mathrm{p}$

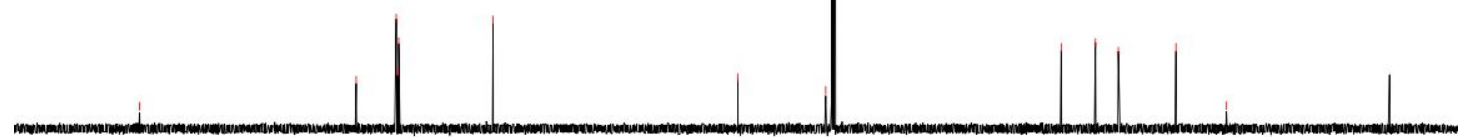

$\begin{array}{llllllllllllllllll}30 & 180 & 170 & 160 & 150 & 140 & 130 & 120 & 110 & 100 \underset{\mathrm{f} 1}{\stackrel{9}{(\mathrm{ppm})}} \mathbf{8 0} & 70 & 60 & 50 & 40 & 30 & 20 & 10 & 0\end{array}$ 

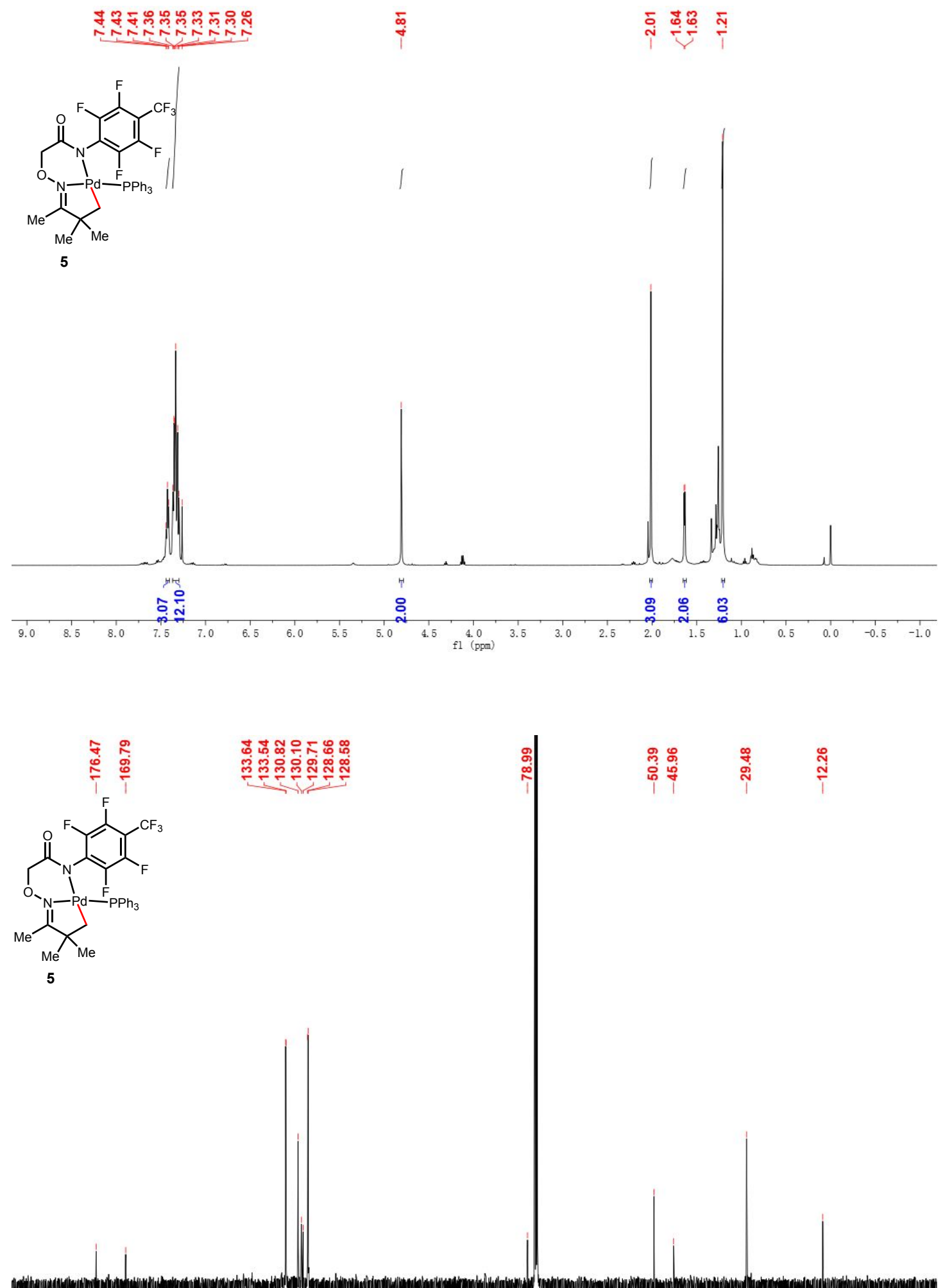

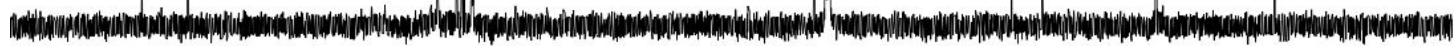

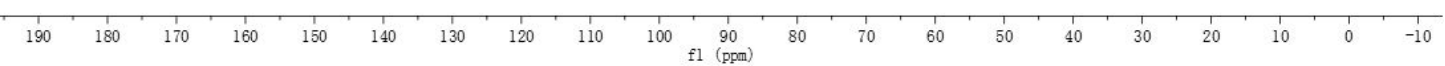


我

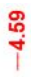

ஓே:

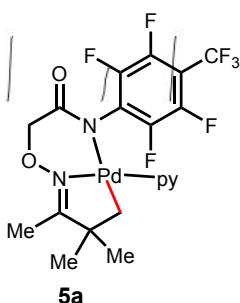<smiles>C=C</smiles>
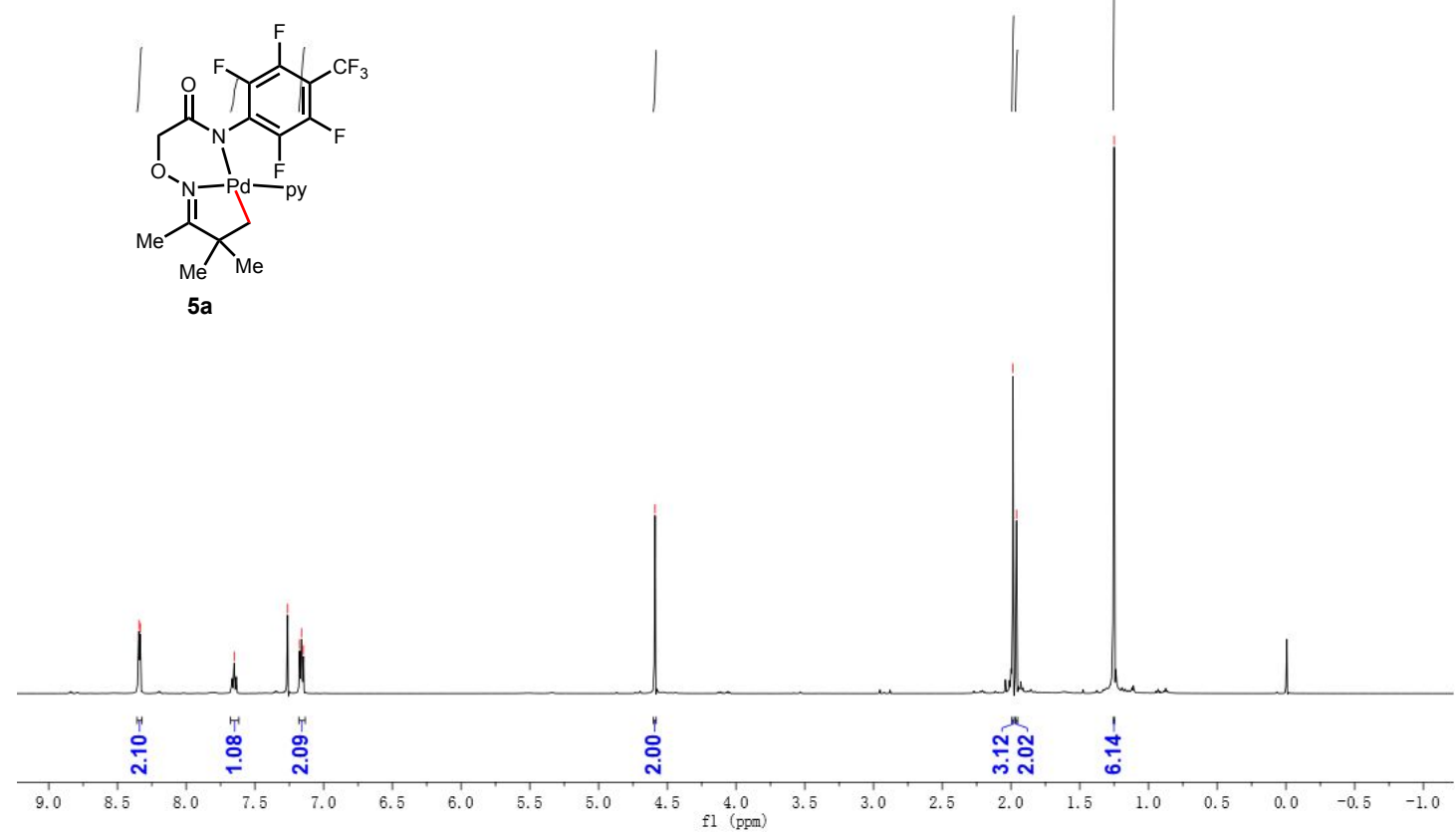

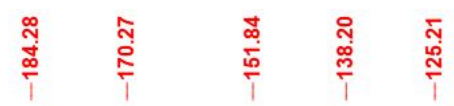

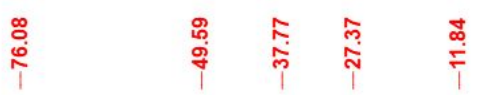

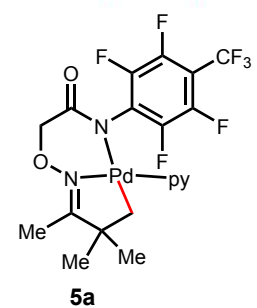

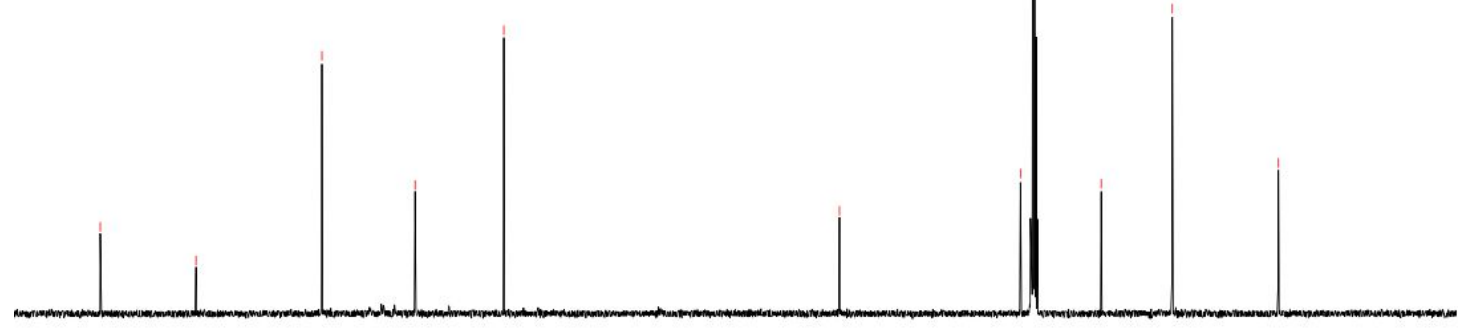

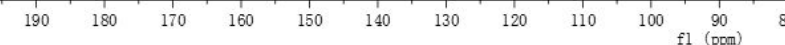

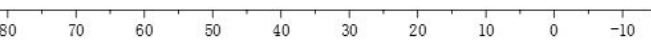



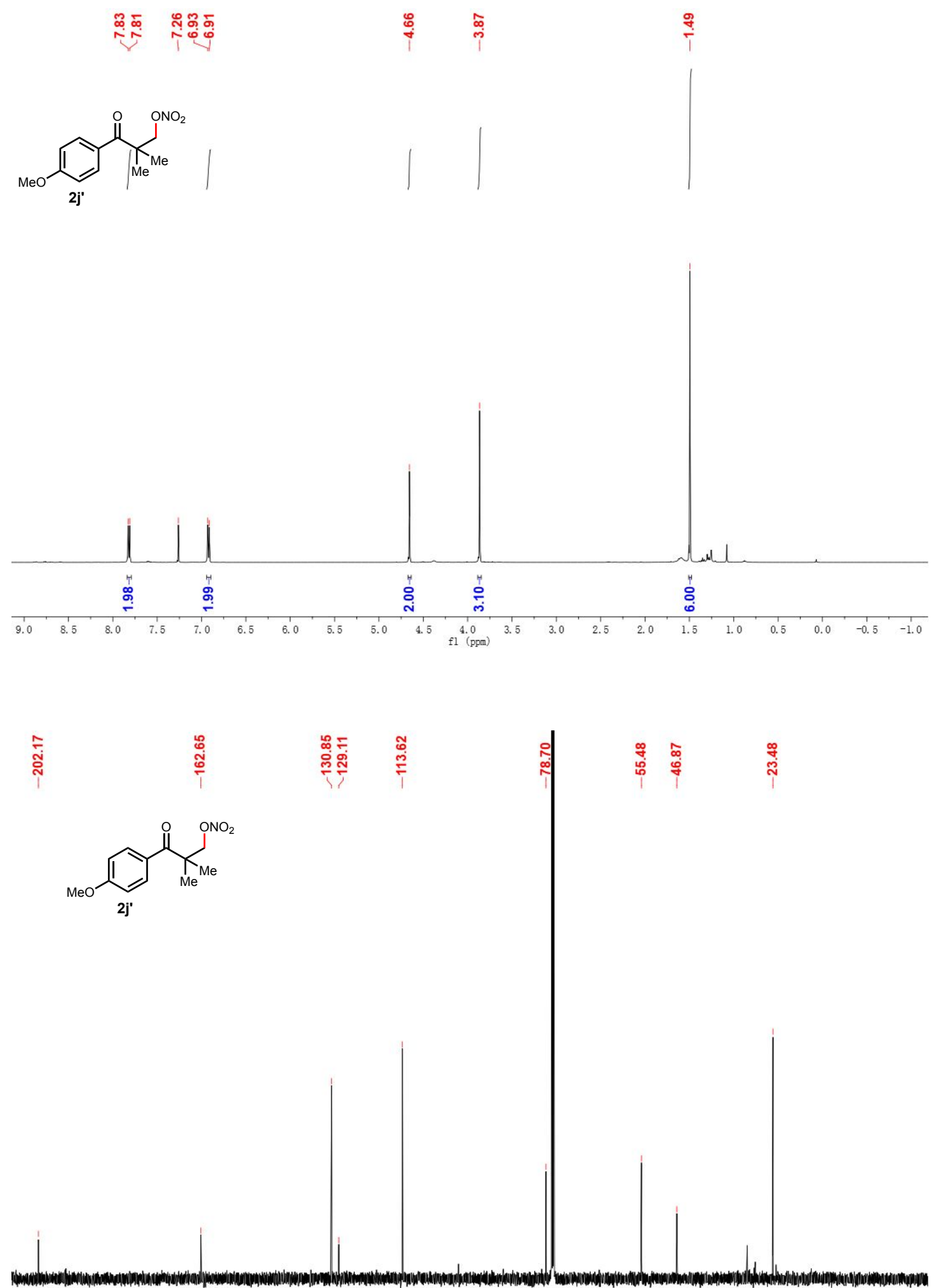

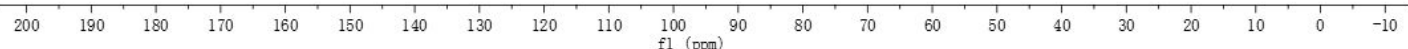



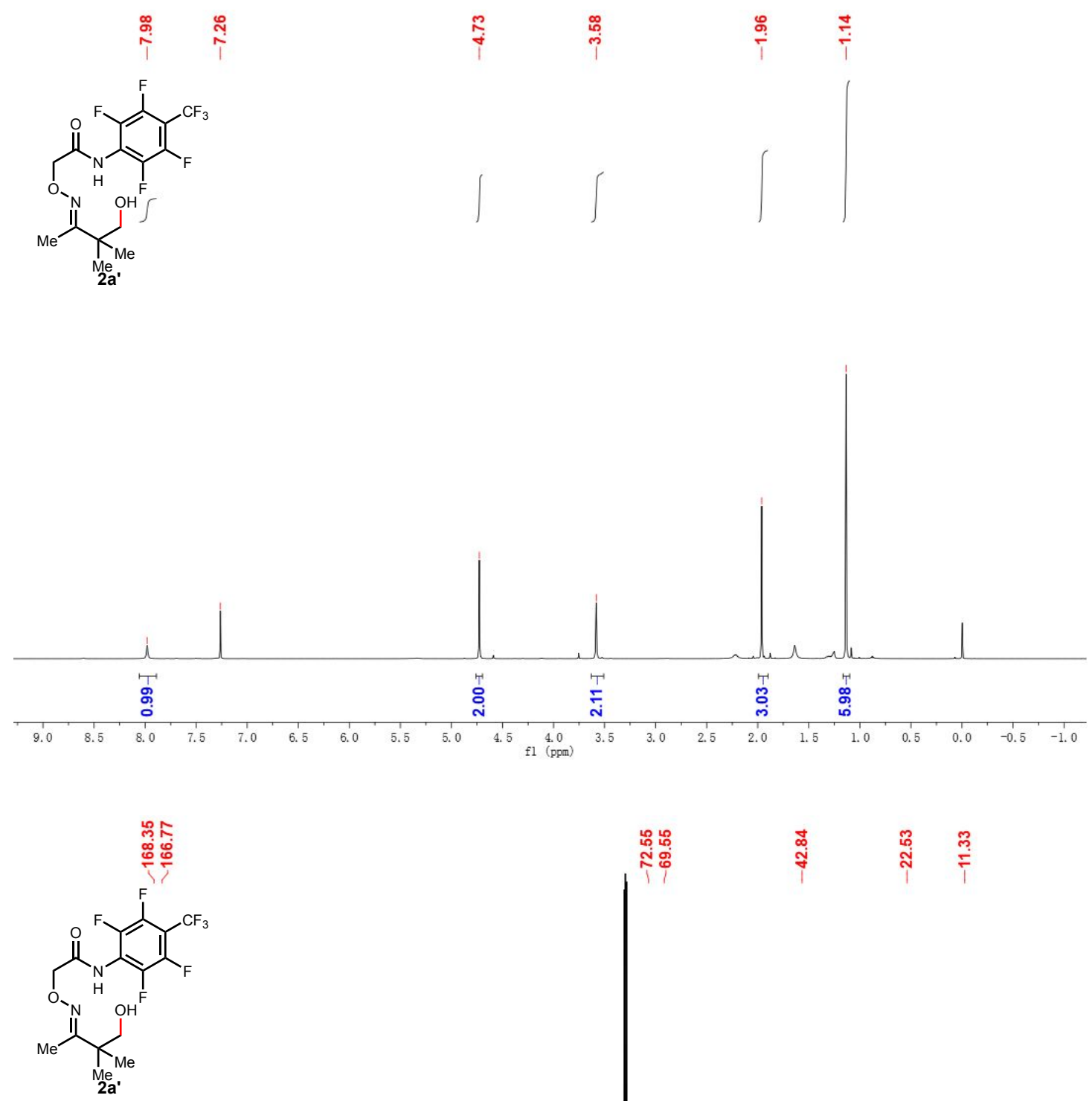

ホั

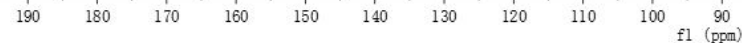

